\title{
Der minderjährige Zeuge im Zivilprozeß
}

Von

Andreas Findeisen

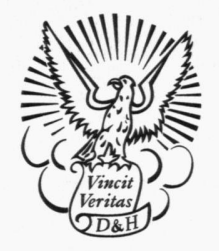

Duncker \& Humblot · Berlin

$$
D i 93 / 47
$$


Die Deutsche Bibliothek - CIP-Einheitsaufnahme

\section{Findeisen, Andreas:}

Der minderjährige Zeuge im Zivilprozess / von Andreas Findeisen. - Berlin : Duncker und Humblot, 1992

(Schriften zum Prozessrecht ; Bd. 111)

Zugl.: Regensburg, Univ., Diss., 1991

ISBN 3-428-07545-5

NE: GT

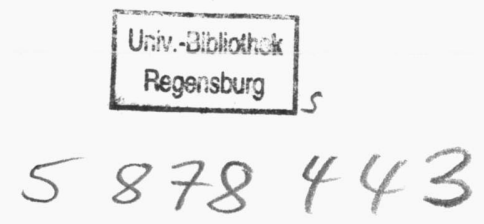

Alle Rechte vorbehalten

(c) 1992 Duncker \& Humblot GmbH, Berlin 41

Fotoprint: Werner Hildebrand, Berlin 65

Printed in Germany

ISSN 0582-0219

ISBN 3-428-07545-5 


\section{Vorwort}

Der minderjährige Zeuge hat Rechtsprechung und Literatur bisher vorwiegend im Zusammenhang mit Strafverfahren beschäftigt. Dabei beschränkte sich der Blick für die Besonderheiten auf die Ausübung von Zeugnisverweigerungsrechten. Eine gesetzliche Regelung findet sich bezeichnenderweise nur in $\$ 52$ II StPO.

Der Minderjährigenschutz ist eine allgemein anerkannte Notwendigkeit. Der Schutz des Zeugen, der nicht nur Beweismittel, sondern auch Person mit eigenen Grundrechten ist, wird dagegen erst in jüngerer Zeit diskutiert und vom Gesetzgeber umgesetzt.

Die zweifache Schutzbedürftigkeit kindlicher und jugendlicher Zeugen wird nicht nur bei Bestehen eines Zeugnisverweigerungsrechtes relevant. Sie beeinflußt schon Entstehen und Inhalt der Zeugenpflichten und setzt sich in der Notwendigkeit eines vormundschaftsgerichtlichen Verfahrens fort.

Die vorliegende Arbeit soll darlegen, inwieweit die Anforderungen des Minderjährigenschutzes die Rechte und Pflichten, die für erwachsene Zeugen konzipiert wurden, modifizieren. Dabei wird zunächst die Zeugeneignung und die gegenläufigen Beweisinteressen der Parteien behandelt; im zweiten Abschnitt wird die Stellung des Minderjährigen im Verfahren untersucht. Abschließend ist auf das vormundschaftsgerichtliche Verfahren einzugehen, das bei Beteiligung eines Sorgeberechtigten am Zivilverfahren durchzuführen ist.

Für die Anregung zu dem Gegenstand der Untersuchung bedanke ich mich bei Herrn Prof.Dr.E. Schumann, Universität Regensburg, der mich im Rahmen des Doktorandenseminars mit wertvollen Hinweisen unterstützte.

Regensburg, Mai 1992

Andreas Findeisen 



\section{Inhalt}

Erster Abschnitt

Der Zeugenbeweisantrag bei einem minderjährigen Zeugen

$\S 1$ Der Minderjährige

\& 2 Die Zeugnisfähigkeit ................................................................................................ 21

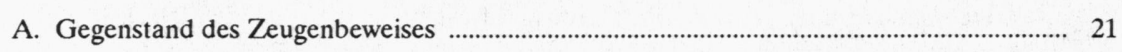

B. Die gesetzliche Regelung der Zeugnisfähigkeit ................................................................. 22

§ 3 Die Beweisantretung beim Zeugenbeweis .................................................................... 25

A. Der Beweisantrag .......................................................................................................... 25

B. Die Ablehnung eines Beweisantrages wegen der Ungeeignetheit eines Zeugen ............. 26

I. Gesetzliche Regelung in der Straf- und der Zivilprozeßordnung ............................. 26

1. Ablehnung von Beweisanträgen im Strafprozeßrecht ............................................... 26

2. Keine Regelung in der Zivilprozeßordnung ........................................................... 27

II. Das "Recht auf Beweis" und der Standpunkt der Rechtsprechung ........................... 28

1. Beweisantrag und rechtliches Gehör ......................................................................... 28

2. Entsprechende Anwendung des § 244 III StPO ….............................................. 30

III. Die Minderjährigkeit als Grund fehlender Eignung................................................... 31

1. Zeugeneignung und Beweiswürdigung ................................................................ 31

2. Der Einfluß natürlicher Grenzen der Wahrnehmungs- und Aussagefähigkeit .... 32

a) Bestimmung der Zeugeneignung im Schrifttum .............................................. 32

b) Auswirkungen fehlender Zeugeneignung ....................................................... 33

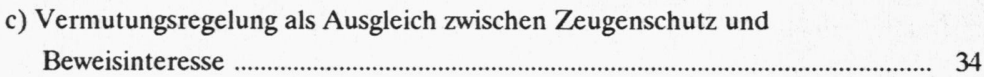

IV. Glaubwürdigkeits- und Eignungsgutachten ............................................................ 37

C. Rechtsbehelfe gegen die Entscheidung über einen Beweisantrag ................................... 38

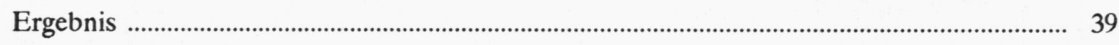




\section{Zweiter Abschnitt \\ Die Stellung des Minderjährigen als Zeuge im Zivilprozeß}

§ 4 Die Ladung des minderjährigen Zeugen ................................................................... 41

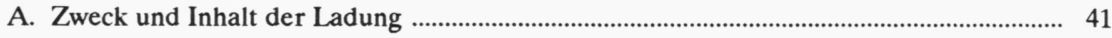

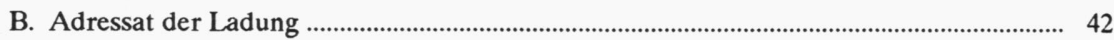

I. Der Minderjährige als Ladungsadressat ................................................................. 42

II. Ladung der gesetzlichen Vertreter ................................................................................. 43

1. Die entsprechende Anwendung des $\S 171$ ZPO ..................................................... 43

2. Einsichtsfähigkeit als bestimmendes Merkmal ........................................................... 44

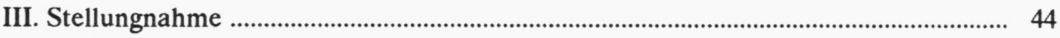

1. Der Minderjährige als alleiniger Ladungsadressat ................................................. 44

2. Die Benachrichtigung der gesetzlichen Vertreter ................................................. 45

3. Hinweispflicht der Parteien ..................................................................................... 47

C. Ordnungsmittel bei Nichterscheinen .............................................................................. 48

I. Darstellung und Verwirkung der Ordnungsmittel ................................................... 48

II. Die Verhängung von Ordnungsmitteln gegen einen Minderjährigen ........................ 49

1. Ordnungsmittel und Kostentragung ........................................................................... 49

2. Die zwangsweise Vorführung .............................................................................. 52

III. Ordnungsmittel gegen die gesetzlichen Vertreter .................................................. 53

IV. Nachträgliche Entschuldigung gemäß § 381 ZPO _.................................................. 55

V. Beschwerde gegen die Verhängung von Ordnungsmitteln ...................................... 56

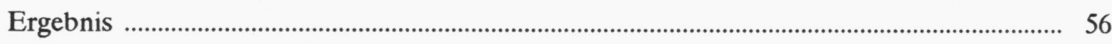

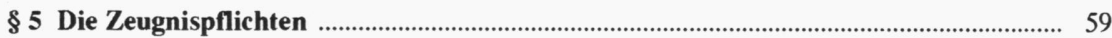

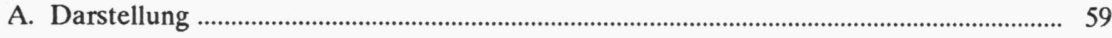

I. Die Erscheinenspflicht und das Aufenthaltsbestimmungsrecht

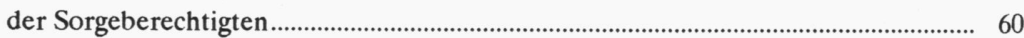

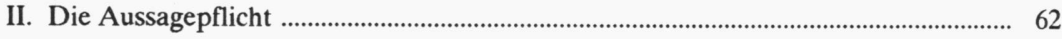

1. Inhalt und Umfang der Ausagepflicht ...................................................................... 62

2. Befreiung von der Aussagepflicht bei fehlender Verstandesreife ........................... 62

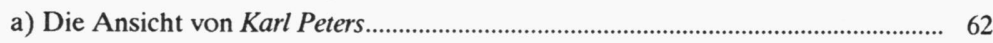

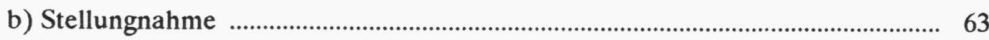


III. Die Eidespflicht

B. Die Weigerungsrechte als Einreden gegen Zeugnispflichten

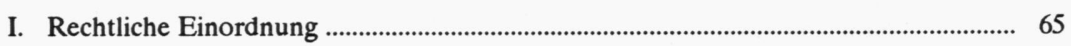

1. Die Zeugnisverweigerungsrechte ............................................................................ 65

2. Das Eidesverweigerungsrecht .................................................................................. 68

II. Gesetzliche Regelung in der Zivilprozeßordnung ................................................... 69

1. Die Zeugenmündigkeit im geltenden Recht nach der Auffassung von F. W. Bosch ........................................................................................................ 69

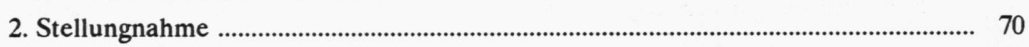

III. Gesetzliche Regelung in der Strafprozeßordnung, insbesondere § 52 II StPO ......... 71

C. Die Ausübung von Zeugniszwang gegenüber einem minderjährigen Zeugen ................. 74

I. Ordnungsmittel gemäß § 390 I ZPO ......................................................................... 74

II. Die Erzwingungshaft nach § 390 II ZPO ................................................................ 75

1. Die Verhängung gegen einen schuldunfähigen Zeugen .......................................... 75

2. Zwangsmittel gegen die gesetzlichen Vertreter ....................................................... 77

III. Die Beschwerde gegen die Verhängung von Ordnungsmitteln ................................. 77

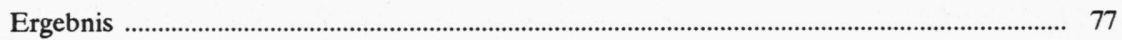

\section{§ 6 Die Ausübung des Zeugnisverweigerungsrechts im Zivilprozeß durch einen}

Minderjährigen ……………………………………………............................................... 79

A. Einsichtsfähigkeit als Ausübungsvoraussetzung ……....................................................... 79

B. Minderjährige mit ausreichender Verstandesreife …......................................................... 83

C. Minderjährige ohne ausreichende Verstandesreife ……................................................... 85

I. Wegfall des Zeugnisverweigerungsrechts wegen fehlendem Konfliktsempfinden? ... 85

II. Beteiligung keines Elternteils am Verfahren .............................................................. 87

1. Zeugnisverweigerung und Zustimmungserfordernis ............................................... 87

a) Personensorgerecht und Höchstpersönlichkeit der Zeugnisverweigerung ...... 87

b) Aussagebereitschaft als Voraussetzung der Zustimmung der gesetzlichen Vertreter ................................................................................................................... 89

c) Zeitpunkt der Zustimmung ............................................................................. 90

2. Zustimmungsbefugnis bei Meinungsverschiedenheiten der Eltern ......................... 92

a) Entscheidung des Vormundschaftsgerichts gemäß § 1628 BGB? ..................... 92

b) Vormundschaftsgerichtliche Maßnahmen nach $\S 1666$ BGB? .......................... 94 
III. Beteiligung beider Elternteile am Verfahren ................................................................ 95

1. Ausschluß der gesetzlichen Vertreter ........................................................................ 95

a) Darstellung des Streitstandes .......................................................................... 95

b) Stellungnahme ………......................................................................................... 96

2. Bestellung eines Pflegers durch das Vormundschaftsgericht ................................. 99

IV. Beteiligung eines Elternteils am Verfahren ............................................................ 99

1. Gemeinsame Sorgerechtsausübung beider Eltern ................................................ 99

2. Sorgerechtsausübung durch einen Elternteil ............................................................ 101

D. Ausübung des Zeugnisverweigerungsrechts ......................................................................... 104

I. Die Erklärung der Zeugnisverweigerung durch einen einsichtsfähigen Minderjährigen

II. Ausübung durch einen nicht Einsichtsfähigen .......................................................... 105

III. Ausschluß der nicht am Verfahren beteiligten gesetzlichen Vertreter bei konkreter Interessenkollision ................................................................................... 106

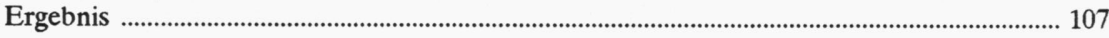

\& 7 Die Belehrung des minderjährigen Zeugen ………………............................................... 109

A. Gesetzliche Regelung ........................................................................................................... 109

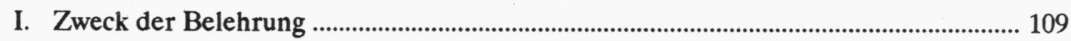

II. Belehrung der Ausübungsbefugten ............................................................................. 110

1. Belehrung des Minderjährigen selbst ....................................................................... 110

2. Belehrung der gesetzlichen Vertreter bei fehlender Einsichtsfähigkeit des Zeugen ............................................................................................................. 112

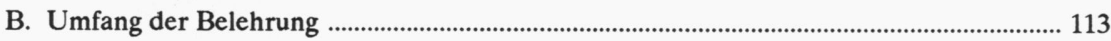

\& 8 Die Vernehmung des Minderjährigen durch das Gericht ................................................. 115

A. Prozessuale Fürsorgepflicht des Gerichts .......................................................................... 115

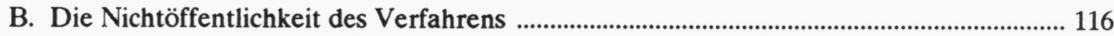

I. Ausschluß der Öffentlichkeit ..................................................................................... 116

1. Voraussetzungen und prozessualer Anspruch auf Ausschließung ........................... 116

2. Beantragung durch den minderjährigen Zeugen .................................................... 118

3. Antragsrecht der gesetzlichen Vertreter ................................................................ 118

4. Antragsrecht der am Verfahren beteiligten Sorgeberechtigten ............................. 118

5. Anwesenheitsrecht der nicht am Verfahren beteiligten gesetzlichen Vertreter .... 119 
II. Ausschluß der Parteien ................................................................................................ 119

C. Durchführung der Vernehmung .......................................................................................... 121

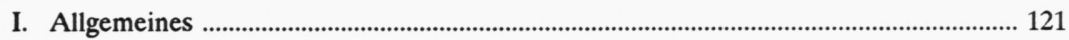

II. Das Fragerecht der Parteien .......................................................................................... 122

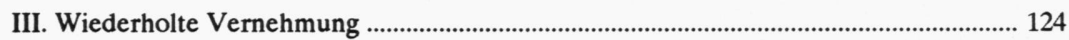

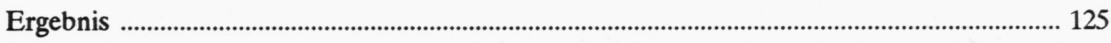

\& 9 Die Verwertung einer prozeßordnungswidrig gewonnenen Aussage ................................ 127

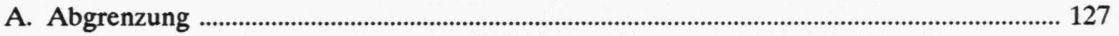

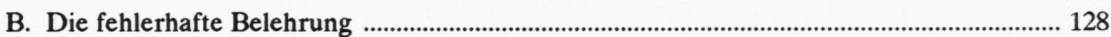

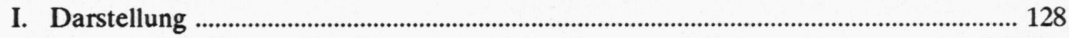

II. Verfahrensrechtliche Folgen der Falschbelehrung ………............................................ 128

1. Nachholung der Belehrung ..................................................................................... 128

2. Verwertungsverbot .................................................................................................... 129

3. Heilung des Verfahrensfehlers .......................................................................... 130

a) Parteiherrschaft und Prozeßökonomie .............................................................. 130

b) Rügeverzicht und Zeugenschutz ........................................................................ 131

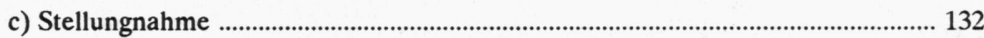

C. Verkennung der Einsichtsfähigkeit und der Zustimmungsbefugnis .................................. 133

D. Verletzung der Vorschriften über die Öffentlichkeit des Verfahrens ................................. 133

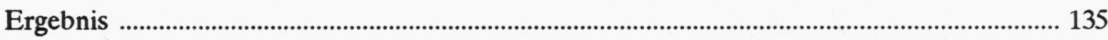

§ 10 Zwischenstreit über das Vorliegen eines Zeugnisverweigerungsrechts und Rechtsmittel des Zeugen

A. Der Zwischenstreit über die Berechtigung der Zeugnisverweigerung

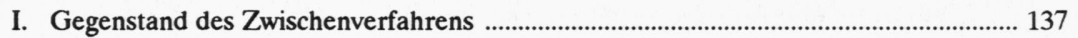

II. Die Durchführung des Zwischenverfahrens bei Beteiligung eines einsichtsfähigen Zeugen

1. Befugnis zur selbständigen Entscheidung ................................................................ 139

2. Die Prozeßfähigkeit Minderjähriger im Zwischenverfahren ................................. 139

a) Verpflichtungsfähigkeit und prozessuale "Wehrbefugnis" ................................. 139

b) Entscheidungsfähigkeit einsichtsfähiger Zeugen ............................................... 140 
III. Das Zwischenverfahren gegen einen nichteinsichtsfähigen Zeugen

1. Aussagepflicht trotz Aussageunwilligkeit und Zustimmungsverweigerung

2. Unzulässigkeit des Zwischenverfahrens wegen fehlendem Rechtsschutzbedürfnis?

a) Rechtsschutzbedürfnis als Verfahrensvoraussetzung

b) Das Rechtsschutzbedürfnis und der Zweck des Zwischenverfahrens

B. Das Rechtsmittel der Beschwerde

I. Gegenstand der Beschwerde durch einen Zeugen 146

II. Die Einlegung der Beschwerde durch einen minderjährigen Zeugen 146

1. Der schuld- und einsichtsfähige Zeuge 146

2. Die Beschwerde des nicht einsichtsfähigen Minderjährigen 147

III. Beschwerderecht der gesetzlichen Vertreter 149

\section{Dritter Abschnitt \\ Das Verfahren vor dem Vormundschaftsgericht bei Verhinderung der gesetzlichen Vertreter}

$\S 11$ Die Entscheidung des Vormundschaftsgerichts

A. Allgemeines

I. Zuständigkeit und Verfahren

II. Die Anrufung des Vormundschaftsgerichts

III. Auswirkungen auf den Zivilprozeß

B. Fürsorgebedürfnis als Voraussetzung der Pflegschaftsanordnung

I. Notwendigkeit der Pflegerbestellung

1. Fehlende Verstandesreife

2. Aussagebereitschaft des minderjährigen Zeugen

3. Rücknahme des Beweisantrages

II. Die vorsorgliche Bestellung eines Pflegers

III. Bindung des Vormundschaftsgerichts 
C. Anhörung der Beteiligten

I. Rechtliches Gehör und Anhörung im Verfahren der freiwilligen Gerichtsbarkeit ... 159

II. Anhörung des Minderjährigen 160

1. Gegenstand und Zweck der Anhörung ................................................................ 160

2. Die Durchführung der Anhörung ........................................................................... 161

3. Absehen von der Anhörung gemäß § 50b III FGG .................................................. 161

III. Anhörung der Eltern .................................................................................................... 162

IV. Die Beteiligung der Prozeßparteien am vormundschaftsgerichtlichen Verfahren ... 163

D. Auswahl des Pflegers durch das Vormundschaftsgericht ................................................... 163

I. Die Person des Pflegers ................................................................................................... 163

II. Die Bestellung des Pflegers ........................................................................................ 165

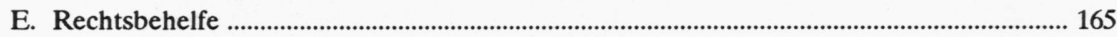

I. Beschwerde gegen die Anrufung des Vormundschaftsgerichts ................................... 166

II. Rechtsschutz gegen die Entscheidung des Vormundschaftsgerichts ........................... 166

1. Erinnerungsbefugnis bei abgelehnter Pflegerbestellung ............................................ 166

2. Erinnerungsbefugnis bei angeordneter Pflegerbestellung ......................................... 167

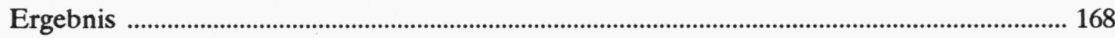

\& 12 Die Rechtsstellung des Ergänzungspflegers ……………………................................... 171

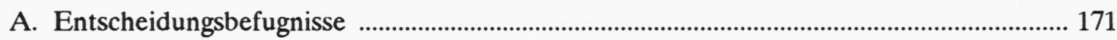

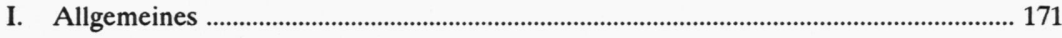

II. Übertragener Wirkungskreis ................................................................................ 172

B. Wirksamkeit der Entscheidung ……………..................................................................... 172

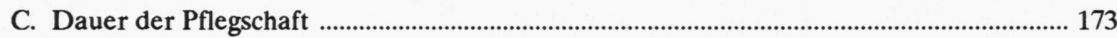

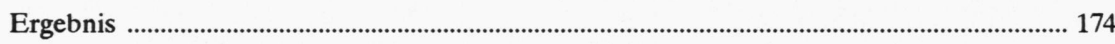

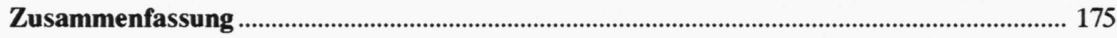

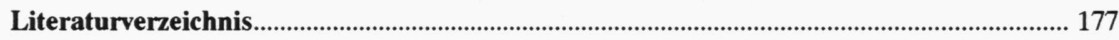





$\begin{array}{ll}\text { Am angegebenen Ort } & \text { Abzungen } \\ \text { Anchiv für die civilistische Praxis } \\ \text { Anm. }\end{array}$


DRiZ

Einf.

FamRZ

Fn

FS

GA

GS

JA

JGG

JR

JurBüro

JuS

JW

LG

MDR

M.E.

M.w.N.

NJW

NJW-RR

NStZ

OLG

OLGZ

OWiG

RdJB
Deutsche Richterzeitung

\section{Einführung}

Zeitschrift für das gesamte

Familienrecht

Fußnote

Festschrift

Goldthammers Archiv

Großer Senat

Juristische Arbeitsblätter

Jugendgerichtsgesetz

Juristische Rundschau

Das juristische Büro

Juristische Schulung

Juristische Wochenschrift

Landgericht

Monatsschrift des Deutschen

Rechts

Meines Erachtens

Mit weiteren Nachweisen

Neue Juristische Wochenschrift

Neue Juristische Wochenschrift -

Rechtsprechungsreport

Neue Strafrechtszeitschrift

Oberlandesgericht

Amtliche Sammlung oberlandesgerichtlicher Entscheidungen

Ordnungswidrigkeitengesetz

Recht der Jugend und des

Bildungswesens 
RiStBV

RKEG

Rn

RPfleger

S.

s.

StPO

VersR

Vgl.

Vorbem.

VRS

ZPO

ZRP

ZZP
Richtlinien für das Strafverfahren und das Bußgeldverfahren in der ab 1.10.1988 geltenden Fassung

Gesetz über die religiöse

Kindererziehung

Randnummer

Der deutsche Rechtspfleger

Seite

Siehe

Strafprozeßordnung

Zeitschrift für Versicherungsrecht

Vergleiche

Vorbemerkung

Verkehrsrechtssammlung

Zivilprozeßordnung

Zeitschrift für Rechtspolitik

Zeitschrift für Zivilprozeßrecht 



\author{
Erster Abschnitt
}

\title{
Der Zeugenbeweisantrag bei einem minderjährigen Zeugen
}

\section{§ 1 Der Minderjährige}

Der Begriff des Minderjährigen ist weder im Zivilprozeßrecht noch im Bürgerlichem Recht legal definiert. Allerdings bestimmt § 2 BGB den Eintritt der Volljährigkeit auf die Vollendung des 18. Lebensjahres. Im Umkehrschlu $\beta^{1}$ kann daher jede natürliche Person vor Erreichen dieser Altersstufe als minderjährig bezeichnet werden.

Mit dieser Eingrenzung sind jedoch noch keine Feststellungen hinsichtlich der verfahrensrechtlichen Stellung des Minderjährigen und hier insbesondere im Hinblick auf seine Position als Zeuge verbunden. Da es dabei stets um die Ausübung prozessualer Rechte und Pflichten geht ${ }^{2}$, richtet sich die Beurteilung allein nach dem Prozeßrecht. Nur soweit dieses auf das materielle Recht verweist oder keine eigene Regelung enthält, kommt ein Rückgriff auf materiell-rechtliche Vorschriften in Betracht.

Nach der Legaldefinition des $\S 52$ ZPO ist eine Person insoweit prozeßfähig, als sie sich selbständig durch Verträge verpflichten kann. Die Prozeßfähigkeit ist zugleich Voraussetzung für die Fähigkeit zur wirksamen Vornahme von Prozeßhandlungen ${ }^{3}$. Es handelt sich hier nicht um eine

\footnotetext{
${ }^{1}$ Moritz, S. 41.

${ }^{2}$ So bleiben etwa nach materiellem Recht begründete Auskunftspflichten außer Betracht, weil diese unabhängig von der öffentlich-rechtlichen Zeugnispflicht bestehen und diese auch nicht beeinflussen.

${ }^{3}$ Rosenberg-Schwab, § 44 III 1 b; Stein-Jonas-Leipold, §51 Rn 8; Thomas-Putzo, § 51 Anm. II; Zöller-Vollkommer, $52 \mathrm{Rn} 1 \mathrm{f}$. Zur Bedeutung der Prozeßhandlungsfähigkeit bei der Ausübung eines Zeugnisverweigerungsrechtes s.u. § 5 B I 1.
} 
eigenständige Bestimmung, da die Frage der Verpflichtungsfähigkeit nach Bürgerlichem Recht zu beantworten ist ${ }^{4}$. Demnach ist jeder voll Geschäftsfähige verpflichtungs- und damit auch prozeßfähig; umgekehrt ist jede nicht voll geschäftsfähige Person prozeßunfähig, da die ZPO keine beschränkte Prozeßähigkeit kennt ${ }^{5}$.

Genießt ein Minderjähriger für einen speziellen Lebensbereich nach $\S \S 112,113$ BGB die volle Geschäftsfähigkeit bereits vor Vollendung des 18. Lebensjahres, so ist er innerhalb dieses Bereiches auch voll prozeßfähig ${ }^{6}$. Für gesetzliche Vertretung bleibt hier, auch soweit es Prozeßhandlungen betrifft, kein Raum?

Als Minderjähriger wird im weiteren Verlauf daher jeder nicht voll $\mathrm{Ge}$ schäftsfähige bezeichnet, mithin jede natürliche Person vor Vollendung des 18. Lebensjahres unter Ausschluß derer, die partiell einem bereits Volljährigen gleichstehen, soweit die Zeugenaussage mit diesem Bereich in Zusammenhang steht.

\footnotetext{
${ }^{4}$ Rosenberg-Schwab, § 44 I; Thomas-Putzo, §52 Anm. 1.

${ }^{5}$ Baumbach-Lauterbach-Hartmann, §51 Anm. 1; Rosenberg-Schwab, §44 II 2; Stein-JonasLeipold, §51 Rn 2 f. Zu den notwendigen Einschränkungen des §52 ZPO wird noch im Zusammenhang mit der Zwischenstreitigkeit gem. $\S 387 \mathrm{f} \mathrm{ZPO} \mathrm{und} \mathrm{der} \mathrm{Beschwerdebefugnis}$ einzugehen sein, dazu unten $\S 10$.
}

${ }^{6}$ Dehmer, S. 64 ff; Kahlke, ZZP 100, 10, 13; Stein-Jonas-Leipold, §52 Rn 4 ff; Thomas-Putzo, $\S 52$ Anm. 1 b; Zölier-Vollkommer, §52 Rn 4. Ebenso Grundmann, Der Minderjährige im Zivilprozeß, 1980, S. $18 \mathrm{ff}$ - Grundmann spricht von relativer Prozeßfähigkeit; m.E. treffender wäre von partieller Prozeßfähigkeit zu sprechen (entsprechend wie bei der Geschäftsfähigkeit, vgl. Flume, Allgemeiner Teil des Bürgerlichen Rechts, 2. Band: Das Rechtsgeschäft, §13, 4 und 5). Wie hier Bruns, Rn 53.

${ }^{7}$ Schefold, AcP 94 (1903), 305 f. 


\section{§ 2 Die Zeugnisfähigkeit}

\section{A. Gegenstand des Zeugenbeweises}

Der Zeuge ist eine am konkreten anhängigen Verfahren nicht als Partei beteiligte natürliche Person. Als Zeugen scheiden neben den Parteien auch die gesetzlichen Vertreter aus, die den Prozeß im Namen eines prozeßunfähigen Vertretenen führen ${ }^{1}$.

Gegenstand des Zeugenbeweises sind eigene, außerhalb des Prozesses gemachte Wahrnehmungen über vergangene Tatsachen oder Zustände ${ }^{2}$. Abzugrenzen ist der Zeuge vom sachverständigen Zeugen im Sinne des $\S 414 \mathrm{ZPO}$ und vom Sachverständigen ${ }^{3}$.

Die Bedeutung des Zeugenbeweises wird sehr unterschiedlich beurteilt; die Gefahr bewußt und unbewußt unrichtiger oder unvollständiger Aussagen läßt die weit verbreitete Zurückhaltung bei der Beweiswürdigung zugunsten anderer, zuverlässigerer Beweismittel verständlich erscheinen ${ }^{4}$. Bei Aussagen minderjähriger Zeugen dürfte die Skepsis sogar noch zunehmen, je niedriger das Alter ist ${ }^{5}$. Umgekehrt kann aber nicht übersehen werden,

${ }^{1} B G H$, FamRZ 1964, 150 ff; Lent, ZZP 52, 14 ff; Bergerfurth, JZ 1971, Rn 182 b; RosenbergSchwab, § 123 II 1.

${ }^{2}$ BGH, DRiZ 1974, 27; LG Bamberg, VersR 1984, 49; Rosenberg-Schwab, § 123 I; Stein-JonasSchumann, Vor § 373 Rn 17; Thomas-Putzo, Vor § 373 Anm. 1; Wieczorek, § 373 Anm. A II; Zöller-Stephan, § $373 \mathrm{Rn} 1$.

${ }^{3}$ Dazu Stein-Jonas-Schumann, Vor § 373 Rn 17; Bruns, Rn 182 c.

${ }^{4}$ Baumbach-Lauterbach-Hartmann, Übers. 373 Anm. 1 C b a.E.; Bull, DRiZ 1972, 205; Heusler; AcP 62, 209, 304 ff (zum Recht vor 1879); Knippel, MDR 1980, 112, 113; MusielakStadler, Rn 43,44; Reinicke, MDR 1986, 630 ff; Schneider, MDR 1965, 14 ff; Stein-Jonas-Schumann, Vor § $373 \mathrm{Rn} 21$.

5 Döhring, Die Erforschung des Sachverhaltes im Prozess, 1964, S. 85, 86 bezügl. Kinderaussagen; Krönig, Die Kunst der Beweiserhebung im ZivilprozeB, S. 82; Prahm, Die ärtzlichpsychologische Beurteilung der Glaubwürdigkeit Minderjähriger und ihre Berücksichtigung im Gerichtsverfahren, 1972, S. 54 ff. Zur Entwicklung im Strafprozeß Orlowsky, S. 6 ff; Als- 
daß der Zeuge das häufigste und damit das praktisch bedeutsamste Beweismittel ist ${ }^{6}$.

\section{B. Die gesetzliche Regelung der Zeugnisfähigkeit}

Die Zivilprozeßordnung kennt in den Vorschriften zum Zeugenbeweis keine Regelung, die eine wie auch immer geartete Eignungsvoraussetzung ausdrücklich normiert ${ }^{7}$. Lediglich $\S 393 \mathrm{ZPO}$ nennt in Übereinstimmung mit $\S 455$ II ZPO ein Mindestalter von 16 Jahren, mit dem die Eidesmündigkeit beginnt. Daraus leitet die Literatur nahezu einhellig und wohl in Übereinstimmung mit der Rechtsprechung, in der das Problem - soweit ersichtlich bisher kaum Niederschlag gefunden hat ${ }^{8}$, im Umkehrschluß ab, daß die ZPO keine zeugnisunfähigen Personen kennt. Von den Parteien und gesetzlichen Vertretern abgesehen ist daher jedermann unterschiedslos als Zeuge zugelassen?.

Als allgemeiner Grundsatz des Prozeßrechtes gilt somit, daß jedermanns Tatsachenkenntnisse für den Prozeß verwendet werden sollen und deshalb der Kreis der Personen, die als Zeugen vernommen werden können, nicht ohne zwingende Gründe eingeschränkt werden darf ${ }^{10}$. Dies ergibt sich auch daraus, daß jede gesetzliche Einschränkung der Zeugnisfähigkeit das Recht

berg/Nüse/Meyer, Der Beweisantrag im Strafprozeß, S. 701; Stern, DJZ 1908, 51 ff. Ferner auch unten $\S 3 \mathrm{~B}$.

${ }^{6}$ Bull, DRiZ 1972, 205; Döhring, S. 21; Meyke, NJW 1989, 2032 f; Musielak-Stadler; Rn 59; K. Peters, Zeugenlüge, 1939, S. 273 ff; derselbe, Strafprozeß, § 44 III 1 a, b; Schneider, Beweis und Beweiswürdigung, S. 167 ff; ders. in MDR 1964, 14 ff.

${ }^{7}$ Anders z.B. noch bis 1969 das Bürgerliche Recht, das in $\S 2237$ Nr. 1 BGB Minderjährige als Testamentszeugen ausschloß.

${ }^{8}$ Lediglich $B G H$ VersR 1956; BGH VRS 33, 174 ff; zur Geeignetheit des Beweismittels unten $\S 3 \mathrm{~B}$.

${ }^{9}$ Bruns, Rn 183 c; Grundmann, S. 89; Musielak-Stadler; Rn 60; Baumbach-LauterbachHartmann, Übers, § 373 Anm. 2 A; Stein-Jonas-Schumann, Vor § 373 Rn 3; Zöller-Stephan, § $373 \mathrm{Rn} 3$.

${ }^{10}$ So $B A G$, JZ 1973, 58, 59 . 
der Parteien auf den Beweis beschränken und gegebenfalls verletzen könnte ${ }^{11}$.

Dieser Grundsatz ist Folge der Einführung der freien richterlichen Beweiswürdigung, die bereits im ersten Entwurf, dem sog. Hannoverschen Entwurf von 1866 zur Reichscivilprozessordnung enthalten war und die auch in das schließlich angenommene Gesetz von 1879 aufgenommen wurde ${ }^{12}$. Die Freiheit der Beweiswürdigung setzte die Aufhebung der zahlreichen Beweisregeln voraus. Dies war, auch soweit sie die Zeugenaussagen Kinder und Jugendlicher betrafen, in den Beratungen der "Commision zur Schaffung einer allgemeinen Civilprozeßordnung für die deutschen Bundesstaaten", die dem Hannoverschen Entwurf vorausgingen, umstritten. Die Befürworter einer wenigstens teilweisen Beibehaltung der Regelungen über den "testes inhabilis", zu dem auch Kinder und Unmündige gehörten, konnten sich jedoch nicht durchsetzen ${ }^{13}$. Der Versuch, die Zeugnisfähigkeit mit der Eidesfähigkeit zu verbinden, was eine Altersgrenze von 16 Jahren bedeutet hätte, scheiterte ebenfalls. Dem Richter sollte keine Quelle zur Erforschung der Wahrheit verschlossen bleiben.

Das Prinzip der freien Beweiswürdigung wird heute als eine der wichtigsten Errungenschaften der CPO angesehen. Diese Entwicklung zeigt, daß das Fehlen einer Regelung der Zeugnisfähigkeit nicht auf einer Regelungslücke beruht, sondern daß der historische Gesetzgeber eine bewußte Entscheidung getroffen hat, keinen Personenkreis nach abstrakt formulierten persönlichen Eigenschaften auszuschließen.

\footnotetext{
${ }^{11}$ So Walter, NJW 1988, 567 f, der in einem "a-priori-Ausschluß fragwürdiger Beweismittel" eine Verletzung des "Rechts auf Beweis" sieht; zu diesem Begriff auch unten § 3 B I.

${ }^{12}$ Stein-Jonas-Schumann, Einl. Rn 105.

${ }^{13}$ So wurde die im Hannoverschen Entwurf als $\S 314$ zunächst vorgeschlagene Regelung, als Zeugen dürften nicht zugelassen werden "[...] Kinder vor erlangter Fähigkeit, einen Eid oder diesem gleichstehende Versicherung abzuleisten [...]" nicht angenommen (bei Schubert, Protocolle Band 7 S. 2144). Die schließlich nach zweiter Lesung als $\S 329$ CPO angenommene Vorschrift lautete: "Als unzulässige Zeugen sind auf Antrag oder von Amtswegen zu verwerfen: 1) Personen, welche zur Mittheilung ihrer Wahrnehmungen unfähig sind, oder welche zu der Zeit, auf welche sich ihre Aussage beziehen soll, zur Wahrnehmung der zu beweisenden Tatsache geisitg oder körperlich unfähig waren. 2) [...]", abgedruckt bei Schubert, Protocolle Band 218. Zu der damals neuen Regelung Schmidt, Lehrbuch des Deutschen Civilprozessrechts, 1906, S. 63 und S. 475; Wetzell, System des ordentlichen Civilprozesses, 1878, S. 207. Zur historischen Entwicklung auch Bosch, S. 25 ff.
} 



\section{§ 3 Die Beweisantretung beim Zeugenbeweis}

\section{A. Der Beweisantrag}

Der Zeugenbeweis wird nur auf Antrag einer Partei erhoben. Dies ist Ausdruck des Verhandlungsgrundsatzes im Zivilverfahren. Er besagt, daß es die Aufgabe der Parteien ist, dem Gericht den für die Entscheidung des Rechtsstreites notwendigen Tatsachenstoff zu unterbreiten ${ }^{1}$. Diese Obliegenheit können die Parteien durch die gesetzlich vorgesehenen Beweismittel erfüllen.

In diesem Zusammenhang kommt es nicht auf die Frage an, ob es sich bei der Beweisführung um einen förmlichen Antrag oder lediglich um ein Beweiserbieten handelt; nachfolgend wird immer von Beweisantrag gesprochen, wenn es um die von einer Partei veranlaßte Erhebung eines Beweises geht. Die beweispflichtige Partei ${ }^{2}$ genügt der Last, wenn sie für eine behauptete beweisbedürftige Tatsache ein geeignetes, gesetzlich vorgesehenes Beweismittel anbietet.

Für den Zeugenbeweis ist die Beweisantretung in $\S 373 \mathrm{ZPO}$ geregelt. Danach hat die beweisführende Partei den Zeugen einschließlich seiner ladungsfähigen Anschrift und die Tatsachen, über die die Vernehmung erfolgen soll, zu benennen.

\footnotetext{
${ }^{1}$ BVerfGE 52, 131, 153. Auf die Besonderheiten in zivilrechtlichen Verfahrensarten mit Untersuchungsgrundsatz ist hier nicht einzugehen; vgl. dazu allg. Bettermann, ZZP 91, 365, 89 ff; Baumbach-Lauterbach-Hartmann, Einf. § 284 Anm. 2; Stein-Jonas-Schumann, Vor § $128 \mathrm{Rn}$ 75 ff und Rn 91 ff. Die dem Richter eingeräumte Möglichkeit, auch im Verfahren mit Beibringungsgrundsatz Beweis von Amts wegen erheben zu können (z.B. §§ 142 ff, § 287 I 3, § 358a, § $448 \mathrm{ZPO}$ ) kann ebenfalls unberücksichtigt bleiben, da die Zeugenvernehmung immer nur auf Parteiveranlassung angeordnet werden kann, Habscheid, ZZP 96, 306, 309; Schneider, ZZP 75, 173, 177; Stein-Jonas-Leipold, Vor § 128 Rn 78; Teplitzky, JuS 1968, 71, 73; Wieczorek, $\S 373$ Anm. D.

${ }^{2}$ Vgl. Stein-Jonas-Leipold, § 284 Rn 29 ff; Habscheid, ZZP 96, 306, 307 f. Zum Begriff der sog. formellen Beweislast Goldchmidt, ZPR, S. 133.
} 


\section{B. Die Ablehnung eines Beweisantrages wegen der Ungeeignetheit eines Zeugen}

\section{Gesetzliche Regelung in der Straf- und der Zivilprozeßordnung}

\section{Ablehnung von Beweisanträgen im Strafprozeßrecht}

Im Strafverfahren soll der staatliche Strafanspruch auf der Grundlage des historisch wahren Sachverhaltes durchgesetzt werden ${ }^{3}$. Angesichts der weitreichenden Bedeutung des Verfahrens für den Angeklagten genießt die Aufklärung des tatsächlichen Geschehens einen hohen Stellenwert ${ }^{4}$. Die Beweisaufnahme wird nicht zuletzt deswegen von Amts wegen durchgeführt; nach § 244 II StPO hat sie sich auf alle für die Entscheidung bedeutsamen Tatsachen und Beweismittel zu erstrecken 5 .

Trotz der umfassenden Aufklärungspflicht können auch in Strafsachen Beweisanträge zurückgewiesen werden. Es handelt sich um eine Einschränkung der Aufklärungspflicht mit Ausnahmecharakter; sie ist nur unter engen Voraussetzungen zulässig. So kann die Beweiserhebung gemäß § 244 III bis V, § 245 II StPO abgelehnt werden, wenn das Beweisbedürfnis fehlt, das beantragte Beweismittel ungeeignet oder nicht erreichbar ist, oder der Antrag nur mit dem Ziel der Prozeßverschleppung gestellt wird. Daneben rechtfertigen besondere Beweisverbote die Verwerfung eines Beweisantrages $^{6}$.

\footnotetext{
${ }^{3}$ Löwe-Rosenberg-Schäfer, Einl. Kap. 6 Rn 5, 7 ff. In BVerfGE 57, 250, 275 wird darauf verwiesen, daß die Ermittlung des wahren Sachverhaltes ein zentrales Anliegen des Strafverfahrens sei, ohne das das materielle Schuldprinzip nicht verwirklicht werden könne.

${ }^{4}$ BVerfGE 57, 250, 275 und $279=$ NJW 1981, 1719, 1723; BVerfGE 63, 45, $61=$ NJW 1983, 1043, 1045; BVerfG, MDR 1984, 284.

5 Zur Aufklärungspflicht des Strafrichters Kleinknecht/Meyer, § $244 \mathrm{Rn} 10 \mathrm{ff}$; Löwe-Rosenberg-Gollwitzer, § $244 \mathrm{Rn} 38 \mathrm{ff}$.

${ }^{6} \mathrm{Zu}$ den Beweisverboten als Grenze der Wahrheitsforschung Kleinknecht/Meyer, Einl. Rn 50
} ff. 
Die Wahrheitserforschung genießt demnach im Strafprozeß nicht die absolute Priorität, sondern unterliegt im Interesse anderer Verfahrensziele Einschränkungen, vorrangig der Rechtsstaatlichkeit des Verfahrens ${ }^{7}$.

\section{Keine Regelung in der Zivilprozeßordnung}

Die Zivilprozeßordnung kennt keine den § 244 III, § 245 II StPO entsprechenden Regelungen. Daraus kann aber weder der Schluß gezogen werden, der Zivilrichter könne den Umfang der Beweisaufnahme im freien Ermessen bestimmen, noch umgekehrt, die Ablehnung von Beweisanträgen sei stets unzulässig.

Für den Zivilprozeß gilt, daß die Aufdeckung des tatsächlichen oder wirklichen Sachverhaltes nicht das primäre Ziel des Verfahrens ist; es genügt regelmäßig die "formelle Wahrheit". Die Parteien bestimmen im Rahmen des Verhandlungsgrundsatzes darüber, welcher tatsächliche Sachverhalt zur Grundlage der richterlichen Entscheidung gemacht wird.

Das Beweisrecht im Zivilprozeß wird durch die Rechtsprechung konkretisiert, soweit gesetzliche Vorschriften fehlen. Im folgenden soll auf die Möglichkeit der Zurückweisung von Beweisanträgen im Zivilverfahren eingegangen werden, wie sie von Literatur und Rechtsprechung entwickelt und anerkannt wurden. Im Anschluß ist zu untersuchen, unter welchen Voraussetzungen die Vernehmung eines minderjährigen Zeugen gerade wegen seines geringen Alters abgelehnt werden kann.

${ }^{7}$ Zum Strafverfahren BGHSt. 14, 358 f; 19, 326; Kleinknecht/Meyer, Einl. Rn 50; LöweRosenberg-Schäfer, Einl. Kap. 6 Rn 5. Ähnlich Schmidt-Aßmann in Maunz-Dürig, Art. 19 IV Rn 224, der die Grenzen der Beweiserhebung durch die Erfüllung des Rechtsschutzauftrages des Gerichtes gezogen sieht.

${ }^{8}$ Wach, Vorträge, S. 199 bezeichnet die materielle Wahrheit als ein erwünschtes, aber nicht verbürgtes Resultat des Zivilprozesses; Prozeßzweck sei sie nicht. Lenckner, NJW 1965, 321 meint, da $\beta$ der Zivilproze $B$ die Wahrheit nicht um jeden Preis zutage bringen soll. Ähnlich auch Grunsky, Grundlagen, S. 4; Stein-Jonas-Schumann, Einl. Rn 21 f, der darauf hinweist, daß die Wahrheitsfindung nur Mittel zum Zweck ist. Anders aber z.B. E. Peters, ZZP 76, 145 ff, der als Ziel sowohl des Straf- als auch des Zivilprozesses die Erforschung des "historisch wahren Sachverhaltes" bezeichnet. Bosch, DRiZ 1951, 107, 108 räumt der Sachverhaltsaufklärung grundsätzlich den Vorrang vor Individualinteressen ein, schränkt dies jedoch durch die Anerkennung von Zeugnis- und Untersuchungsverweigerungsrechten als Ausnahmen ein. Kritisch zum Grundsatz der "formellen Wahrheit" auch Gaul, AcP 168, 27, 49 ff, der aber ebenfalls feststellt, da $B$ Wahrheitsfindung nicht Wahrheitsfindung um jeden Preis bedeute. 


\section{Das "Recht auf Beweis" und der Standpunkt der Rechtsprechung}

\section{Beweisantrag und rechtliches Gehör}

Obwohl die Findung der materiellen Wahrheit kein eigenständiges Verfahrensziel ist, kann das Gericht - wie im Strafprozeß - über die Beweisanträge der Parteien nicht nach freiem Ermessen entscheiden. Nach Art. 103 I GG hat jedermann einen verfassungsrechtlich garantierten Anspruch auf rechtliches Gehör. Dieser Anspruch umfaßt auch die Gewährleistung, ein rechtliches Begehren durch Tatsachenbehauptungen und Beweisangebote durchzusetzen ${ }^{9}$. Das Recht der Parteien, Beweisanträge zu stellen, effektuiert das rechtliche Gehör, indem es den Nachweis beweiserheblicher Tatsachen und damit die Durchsetzung des Rechts ermöglicht ${ }^{10}$.

In Übereinstimmung mit Habscheid läßt sich von einem "Recht auf Beweis" sprechen, das zur Rechtsschutzgewährung erforderlich ist ${ }^{11}$. Der Anspruch auf rechtliches Gehör schließt die Möglichkeit ein, sich zum Sachverhalt $\mathrm{zu}$ äußern ${ }^{12}$.

Die Zivilgerichte sind daher zur vollständigen Ausschöpfung aller angebotenen Beweise verpflichtet ${ }^{13}$. Die Ablehnung eines Beweisantrages stellt

\footnotetext{
${ }^{9}$ Waldner, S. 68 ff; Walter, S. 302; derselbe, NJW 1988, 567 f spricht hier vom Justizgewährleistungsanspruch, während Rosenberg, §3 I als Grundlage des Beweisantragsrechts den Justizanspruch des Rechtssuchenden sieht, der seinerseits seine Stütze im Rechtsstaatsprinzip des Art. 20 GG findet. Praktische Unterschiede sind damit nicht verbunden, so daß auf die Auseinandersetzung über diese Begriffe hier nicht einzugehen ist.

${ }^{10}$ Dazu Söllner, S. 1 ff; derselbe, MDR 1988, 363 ff; Waldner, S. 68.

${ }^{11}$ Habscheid, ZZP 79, 452, 454 f; derselbe, ZZP 96, 306, 307 ff; Walter, S. 298 ff. Schon das $R G$ sprach von einem Recht der Partei, die einen Zeugen vorgeschlagen hat, "daß dieser nicht ohne Grund das Zeugnis weigere", RGZ 20, 378, 379. Ähnlich auch Rosenberg-Schwab, § 85 III 1; Schwab/Gottwald, Verfassung und ZivilprozeB, S. 56 ff, 59 . Zum Strafverfahren Kleinknecht / Meyer, § 244 Rn 29.

${ }^{12}$ Grunsky, Grundlagen, S. 232 ff; Schmidt-Aßmann in Maunz-Dürig, Art. 103 Abs. I Rn 66 ff.

${ }^{13}$ Stein-Jonas-Leipold, § $284 \mathrm{Rn} 51$ unter Hinweis auf den Justizgewährungsanspruch und die Pflicht zur Wahrheitserforschung. Im Ergebnis übereinstimmend Söllner, S. $72 \mathrm{ff}$ und $80 \mathrm{ff}$, der allerdings auf $\S 286$ I ZPO zurückgreift, weil der Beweisantrag den Inhalt der Verhandlung bestimme; ähnlich Wieczorek, §286 Anm. C; Thomas-Putzo, § 284 Anm. 2; Meyke, NJW 1989, 2032, 2035 und die in Fn 15 genannte Rechtsprechung.
} 
eine Ausnahme dar, ohne daß dieser Grundsatz durch die Geltung des zivilprozessualen Beibringungsgrundsatzes eingeschränkt würde. Sie ist nur unter engen Voraussetzungen zulässig, um eine Entscheidung auf unvollständiger oder unrichtig ermittelter Tatsachengrundlage zu vermeiden ${ }^{14}$. Das Prozeßgericht ist zu einer genauen Prüfung verpflichtet, weil die Nichtberücksichtigung von zulässigen und begründeten Beweisanträgen die beweisführende Partei in ihrem durch Art. 103 I GG garantierten Anspruch auf rechtliches Gehör beeinträchtigt ${ }^{15}$.

Anders als im Strafprozeß bedarf die Ablehnung eines Beweisantrages keiner gesonderten Beschlußfassung des Gerichtes ${ }^{16}$, sondern erfolgt in den Urteilsgründen. Die Nichtberücksichtigung der Beweisantretung muß dort allerdings begründet werden, da es sich um eine Belastung der beweispflichtigen und -führenden Partei handelt ${ }^{17}$. Die Verletzung der Begründungspflicht kann zur Aufhebung des Urteils führen, es sei denn, der Grund für die Ablehnung des Beweisantrages ergibt sich schon aus dem Urteil selbst, etwa wegen der fehlenden Beweisbedürftigkeit.

\footnotetext{
${ }^{14}$ Mes, S. 70 ff; Schneider, ZZP 75, 173, 175; Schönke, FS für Rosenberg, S. 217; E. Peters, ZZP 76, 145 f. Auch in BVerfGE 9, 89, 95 wird festgestellt, daß das rechtliche Gehör Voraussetzung für eine richtige Entscheidung ist.

${ }^{15}$ BVerfGE 42, 364, 367 m.w.N.; 50, 32, 35 f; 60, 247, 249; 60, 250, 252; 65, 305, 307; 69, 141, $143 \mathrm{f}=$ NJW 1986, 833 in ständiger Rechtsprechung; $B G H$ FamRZ 1964, 150, 152. Von der Ablehnung wegen fehlender Eignung des Beweismittels ist die Zurückweisung als Folge einer vorweggenommenen Beweiswürdigung abzugrenzen. Letztere ist stets unzulässig. Dazu auch Habscheid, ZZP 96, 306, 308; Söllner, S. 94 ff; Stein-Jonas-Leipold, § 284 Rn 67 m.w.N.; Thomas-Putzo, §284 Anm. 3 d; Walter, S. 310 ff; Wieczorek, § 286 Anm. C II a. Ebenso zum Strafprozeß Kleinknecht/Meyer, § 244 Rn 29 ff.

${ }^{16}$ Musielak-Stadler, JuS 1979, 721, 724; Stein-Jonas-Leipold, § 284 Rn 85; Teplitzky, JuS 1968, 71, 76. Die Ablehnung des Beweisantrages ist nicht der Nichtvernehmung infolge Zeugnisverweigerung gleichzusetzen. Über die Berechtigung zur Zeugnisverweigerung wird gemä $B$ $\S 387 \mathrm{ZPO}$ entschieden; dazu unten $\S 10 \mathrm{~A}$.

${ }^{17}$ Baumbach-Lauterbach-Hartmann, $\$ 286$ Anm. 3 B i; Goldschmidt, Der ProzeB, S. 455; Schneider, ZZP 75, 173, 183 f; Wieczorek, § 286 Anm. D III a 1; OLG Köln, Jur. Büro 1969, 645. Einschränkend BVerfGE 42, 364, 368 wonach sich das Prozeßgericht nicht ausdrücklich mit jedem Vorbringen der Beteiligten in den Gründen befassen muß.
} 


\section{Entsprechende Anwendung des § 244 III StPO}

Der Bundesgerichtshof greift zur Schließung der Gesetzeslücke auf $\S 244$ III StPO zurück, an den sich auch der Zivilrichter anlehnen dürfe ${ }^{18}$. Für das Zivilprozeßrecht gilt damit ähnlich wie im Strafverfahren, daß die Ablehnung eines Beweisantrages aus rein beweisrechtlichen ${ }^{19}$ oder aus verfahrensrechtlichen ${ }^{20}$ Gründen zulässig ist. Nicht geregelt, im Grundsatz jedoch auf der Grundlage der analogen Anwendung des § 244 III StPO anerkannt, ist die Behandlung ungeeigneter Beweismittel ${ }^{21}$. Es wird daher zu prüfen sein, ob das kindliche Alter eines Zeugen dessen Eignung als Beweismittel ausschließen und damit die Zurückweisung des Beweisantrages rechtfertigen kann.

Ein Zeuge, der bereits berechtigterweise von einem Zeugnisverweigerungsrecht Gebrauch gemacht hat, ist ebenfalls nicht mehr als geeignetes Beweismittel anzusehen ${ }^{22}$, es sei denn, es lägen Anhaltspunkte vor, daß der Zeuge nun nicht mehr auf das Weigerungsrecht zurückgreifen will.

\footnotetext{
${ }^{18} B G H Z$ 53, 245, 259 f "Anastasia"; BGH DRiZ 1959, 252; 1966, 381. Söllner, S. 232 ff nimmt ebenfalls die Analogiefähigkeit von $\S 244$ III, IV StPO an. Kritisch dazu Schneider, ZZP $75,173,180$.

${ }^{19} B G H$, FamRZ 1964, 150 ff; Bruns, Rn 173 b; Rosenberg-Schwab, § 119 I; Schönke, FS für Rosenberg, S. 217, 220 f. Söllner, S. 113 ff; Stein-Jonas-Leipold, § 284 Rn 51 ff; Zöller-Stephan, Vor $\$ 284 \mathrm{Rn} 9 \mathrm{ff}$. So stellen fehlende Beweiserheblichkeit oder -bedürftigkeit Zurückweisungsgründe dar.

${ }^{20}$ Dazu gehört auch die Ablehnung verspäteter Beweisanträge und die erleichterte $\mathrm{Zu}$ rückweisung wiederholter Zeugenvernehmung nach § $398 \mathrm{ZPO}$.

${ }^{21}$ BGH, DRiZ 1974, 27; Rosenberg-Schwab, §§ 113 IV 2, 119 I; Söllner, S. 164 ff; ThomasPutzo, § 284 Anm. 3.

${ }^{22}$ BGHSt. 14, 358, 359; 32, 115, 123 zur Funktion der $\$ \S 52,53$ StPO als Grenze der Aufklärungspflicht des Strafrichters; ferner BGH NJW 1983, 1571 und 1589; NJW 1986, 2261; Löwe-Rosenberg-Gollwitzer, § 244 Rn 188 m.w.N.; Kleinknecht/Meyer, Einl. Rn 18 verweist auf das Rechtsstaatsprinzip.
} 


\section{Die Minderjährigkeit als Grund fehlender Eignung}

\section{Zeugeneignung und Beweiswürdigung}

Die Einführung der Reichscivilprozeßordnung von 1879 brachte die Abschaffung auch der Beweisregeln mit sich, die die Zeugnisfähigkeit von Kindern und Jugendlichen beschränkten ${ }^{23}$. Als Folge davon ist bis in die heutige Zeit allgemein anerkannt, daß auch Minderjährige unabhängig von ihrem Alter und ihrem persönlichen Entwicklungsstand als Zeuge benannt und auch vernommen werden können ${ }^{24}$.

Die Besonderheiten, die die Zeugenvernehmung eines Kindes, darunter auch solcher im Vorschulalter, mit sich bringt, werden nach dieser Ansicht erst im Stadium der Beweiswürdigung berücksichtigt. Der Beweiswert von Kinderaussagen wird jedoch allgemein als gering eingeschätzt ${ }^{25}$, so daß sich die Vernehmung häufig nachträglich als nicht notwendig erweist.

Ob man deshalb von einer "Übersteigerung des Prinzips der Zeugnisfähigkeit für jedermann'" sprechen $\mathbf{k a n n}^{26}$, sei dahingestellt. Es ist jedoch nicht ernstlich zu bezweifeln, daß das Anliegen des Minderjährigenschutzes durch die skizzierte Auffassung nicht ausreichend berücksichtigt wird.

Neben der nur im jeweiligen Einzelfall in Betracht kommenden Möglichkeit der Zeugnisverweigerung ist zu prüfen, ob eine Vernehmung des Minderjährigen notwendig ist oder ob sie wegen der Gefährdung des Kindeswohls unterbleiben soll127. Das Ziel ist, die Vernehmung kindlicher und ju-

\footnotetext{
${ }^{23}$ Dazu oben $\S 2$ B.

${ }^{24}$ Anders wohl nur K. Peters, Gutachten, S. 116 f; derselbe, JR 1970, 308 f zum Strafverfahren. Peers will bei fehlender Einsichtsfähigkeit aus Gründen der Fürsorge und der Fairness ganz auf den Zeugen verzichten. Dazu unten § 5 A II 2.

${ }^{25} \mathrm{Zu}$ dem geringen Beweiswert und dem Bemühen, die Bedeutung des Zeugenbeweises zurückzudrängen etwa Baumbach-Lauterbach-Hartmann, Übers. $\$ 373 \mathrm{Anm} .1 \mathrm{Cb}$ a.E.; Knippel, MDR 1980, 112 f; Schneider, MDR 1965, 14; Schreiber, S. 16 ff. Bezüglich Kinderaussagen weiter einschränkend Döhring, S. 85 f ; Prahm, S. 54 ff; Schimmack, JW 1924, 1667, 1668. Zum Strafverfahren K. Peters, Strafprozeß, § 44 III 1 a; Stern, DJZ 1908, 51,52 f; ders., DJZ 1910, 1001, 1002.

${ }^{26}$ Bosch, S. 54.

${ }^{27}$ Auf diese Gefährdung, die im Strafprozeß in noch stärkerem Maß gegeben ist, weist bereits Römer, RdJB 1971, 205 ff hin. Zum Strafverfahren auch Mönkemöller, S. 423 ff. Mönkemöller, S. 318 f, hält umgekehrt im Zivilprozeß eine Schädigung kindlicher Zeugen aufgrund
} 
gendlicher Zeugen nur ausnahmsweise und unter weitgehendem Ausschluß einer Gefährdung für ihre Entwicklung zuzulassen, ohne auf der anderen Seite aber die berechtigten Beweisinteressen der Prozeßparteien zu vernachlässigen.

\section{Der Einfluß natürlicher Grenzen der Wahrnehmungs- und Aussagefähigkeit}

a) Bestimmung der Zeugeneignung im Schrifttum

Eine verwertbare Aussage setzt voraus, daß der Zeuge die Beweistatsache wahrgenommen und sie in sein Gedächtnis aufgenommen hat; darüberhinaus muß er in der Lage sein, seine Beobachtung auch nach - je nach Länge des zwischen Wahrnehmung und Vernehmung liegenden Zeitraumes - längerer Zeit reproduzieren zu können ${ }^{28}$. Diese Fähigkeiten können bei einem erwachsenem Zeugen regelmäßig unterstellt werden. Bei kindlichen Zeugen sind dagegen entwicklungsbedingte Einschränkungen zu be$\operatorname{achten}^{29}$.

Die Bestimmung der Grenzen der Wahrnehmungs- und Aussagefähigkeit, sei es in Form allgemeiner Erfahrungssätze, sei es im Einzelfall, kann letztlich nur durch sachverständige Psychologen und Psychiater erfolgen. Übereinstimmende Altersgrenzen konnten bisher nicht bestimmt werden. So wird teilweise für eine untere Altersgrenze von sechs Jahren plädier ${ }^{30}$;

des verständnisvollen Zusammenwirkens aller Verfahrensbeteiligten für ausgeschlossen. Seine idealisierende Darstellung des Rechtsstreits in Zivilsachen ist jedoch mit der Prozeßwirklichkeit unvereinbar.

${ }^{28}$ Stein-Jonas-Leipold, $\$ 284 \mathrm{Rn} 69$, der die Vernehmung ausschließt, wenn der Zeuge "eindeutig noch zu jung war, um brauchbare Wahrnehmungen [...] machen zu können". Ferner Thomas-Putzo, § 284 Anm. 3 d; Wieczorek, § 286 Anm. C IV a 3; Söllner, MDR 1988, 363, 364. Das BAG stellte im Jahr 1966 fest, daß Beweisanträge nicht zu berücksichtigen sind, denen ein natürliches Hindernis entgegensteht. Ein natürliches Hindernis wurde auch bei fehlender Aussage- und Wiedergabefähigkeit des Zeugen angenommen, BAG, NJW 1966, 2426; Wieczorek, § 286 Anm. C IV a 3.

${ }^{29}$ Für eine bessere Berücksichtigung aussagenpsychologischer Erkenntnisse bei der Beweiserhebung im Zivilprozeß Schneider, ZZP 75, 173, 193; ders. in Beweis und Beweiswürdigung, S. $167 \mathrm{f}$.

${ }^{30}$ So Detterborn-Fröhlich-Szewccyk, Forensische Psychologie, S. 74. 
andere nehmen an, daß bei normaler geistiger Entwicklung von Kindern unter viereinhalb bis fünf Jahren praktisch keine verwertbare Wiedergabe eines in der Vergangenheit liegenden Vorganges erwartet werden könne ${ }^{31}$. Unter günstigen Bedingungen sei aber auch die Aussage eines zwei- bis vierjährigen Kindes zuverlässig ${ }^{32}$.

Die aussagenpsychologischen Untersuchungen, auf denen diese Altersangaben basieren, wurden im Zusammenhang mit Kinderaussagen in Strafverfahren gemacht. Dabei konzentrierten sich die Erhebungen auf Sexualdelikte, in denen die Kinder als Opfer vernommen worden waren. Die unmittelbare physische und psychische Betroffenheit ist eine wesentliche Ursache für die Aufnahme in das Gedächtnis ${ }^{33}$. Daher kann den Untersuchungsergebnissen für das Zivilverfahren allenfalls indizielle Wirkung zukommen, zumal die Beweistatsachen weder aus dem deliktischen Bereich stammen noch gerade für den kindlichen Zeugen von Bedeutung gewesen sein müs$\operatorname{sen}^{34}$.

\section{b) Auswirkungen fehlender Zeugeneignung}

Die Bedenken, die gegen die unbeschränkte Zeugeneignung anzumelden sind, beruhen darauf, daß die natürlichen Grenzen der Wahrnehmungs- und Aussagefähigkeit nicht hinreichend oder zu spät, nämlich erst nach erfolgter Beweisaufnahme im Rahmen der Beweiswürdigung beachtet werden. Der Schutz minderjähriger Zeugen ist aber nicht gewahrt, wenn die Aussage nach der Vernehmung als ungeeignetes Beweismittel unberücksichtigt

\footnotetext{
${ }^{31}$ Arntzen, DRiZ 1976, 20; zustimmend Kleinknecht/Meyer, Vor § 48 Rn 13.

${ }^{32}$ Undeutsch, Beurteilung der Glaubwürdigkeit von Aussagen, in: Forensische Psychologie, 1967, S. $69 \mathrm{ff}$.

${ }^{33}$ Kleinknecht/Meyer, § 244 Rn 60; Musielak-Stadler, Rn 59 ff; Panhuysen, S. 44 f; Stern, DJZ $1908,51,53$.

${ }^{34}$ Ähnlich Schneider, Beweis und Beweiswürdigung, S. 181, wonach die Wahrnehmungsbereitschaft von der Bedeutung des Vorganges für den Zeugen abhängt. Die Wahrnehmung müsse persönlichkeitsadäquat sein. Dazu auch Schönke, FS für Rosenberg, S. 217, 221. Nach Alsberg/Nüse/Meyer, S. 603 f m.w.N. kann bei einem Kind die Wiedergabe eines Geschehens nicht erwartet werden, wenn dessen Wahrnehmung nicht nur einfache Überlegungen erfordert, wie etwa der Abschluß eines schwierigen Vertrages oder der Inhalt eines längeren Gesprächs.
} 
bleibt. Hier kann nur die Zurückweisung des Beweisantrages Abhilfe schaffen.

Auf der anderen Seite stehen der Verwerfung des Antrages die berechtigten Beweisinteressen der Partei entgegen, der mit dem Beweisverlust auch ein Rechtsverlust droht. Die Schwierigkeit der Feststellung der Eignung kindlicher Zeugen führt zu erheblicher Rechtsunsicherheit. Da aber sowohl der Minderjährigenschutz als auch das Beweisantragsrecht der Parteien hohen Stellenwert genießt, kommt es darauf an, den Konflikt in einer beiden Seiten gerecht werdenden Weise zu lösen.

c) Vermutungsregelung als Ausgleich zwischen Zeugenschutz und Beweisinteresse

Ein Ausgleich der gegenläufigen Interessen kann durch eine Vermutung fehlender Eignung bis zum Erreichen eines bestimmten Alters zugunsten des Kindes erreicht werden. Dieses Alter sollte bei sechs Jahren angesetzt werden. Dadurch wird der Minderjährigenschutz umfassend gewährleistet und der Tatsache Rechnung getragen, daß die Beweisthemen im Zivilprozeß über den deliktischen Bereich hinausgehen und aus der Sicht des Kindes "abstrakt" sein können. Die Interessen der Parteien bleiben gewahrt, denn sie können die Vermutung durch Darlegung der Umstände, die im Einzelfall trotz des geringen Alters eine verwertbare Aussage erwarten lassen, widerlegen ${ }^{35}$. Als besondere Umstände sind z.B. unmittelbare körperliche Betroffenheit oder überdurchschnittliche Entwicklung denkbar; eine erhebliche Rolle spielt auch der zwischen Wahrnehmung und Vernehmung verstrichene Zeitraum ${ }^{36}$.

\footnotetext{
${ }^{35}$ Für den Strafprozeß so auch Kleinknecht/Meyer, § $244 \mathrm{Rn} 60$, wenngleich unter Zugrundelegung einer anderen Altersgrenze; vgl. oben Fn. 31.

${ }^{36}$ BGH 1 StR 334/72 bei Dallinger, MDR 1973, 372; BGH 2 StR 260/82 bei Spiegel, DAR 1983, 203. Die Ablehnung ist danach zulässig, wenn nach "feststehender allgemeiner Lebenserfahrung" unmöglich ist, daß sich der Zeuge an die Beweistatsache erinnern kann. So hält der $B G H$ in den genannten Urteilen die Ablehnung des Beweismittels als völlig ungeeignet bereits dann für rechtens, wenn bis zur Vernehmung ein längerer Zeitraum verstrichen ist; bei einem für den Zeugen belanglosen Vorgang wurde bereits nach nur 1 1/2 Jahren angenommen, es sei hinreichend unwahrscheinlich, daß sich der Zeuge noch daran erinnern könne. Diese Entscheidungen dürften bezüglich des als ausreichend erachteten Zeitraumes nicht verallgemeinerungsfähig sein; einen Überblick über die strafrechtliche Rechtsprechung gibt $\mathrm{Alsberg} / \mathrm{Nüse} /$
} 
Die Annahme einer widerlegbaren Vermutung ist dem Zivilprozeßrecht nicht fremd. Eine vergleichbare Problematik tritt beim "Zeugen vom Hörensagen"37 und beim Indizienbeweis ${ }^{38}$ auf. Hier wird die Ablehnung des Antrages auf Vernehmung eines derartigen Zeugen als zulässig angesehen, wenn der Beweisführer den Antrag nicht auf Verlangen des Gerichts über die Anforderungen des $\S 373$ ZPO hinaus substantiier ${ }^{139}$. Ein sachlicher Unterschied zu einer widerlegbaren Vermutung besteht nicht, sieht man davon ab, $\mathrm{da} ß$ es bei einem kindlichen Zeugen vor Vollendung des sechsten Lebensjahres keiner besonderen Aufforderung des Gerichts bedarf.

Die Einführung einer gesetzlichen Vermutungsregelung ist de lege ferenda aus Gründen der Rechtssicherheit zu befürworten ${ }^{40}$. Bereits de lege lata rechtfertigt der gebotene Minderjährigenschutz erhöhte Anforderungen an einen diesbezüglichen Beweisantrag. Bei sehr jungen Zeugen dürfte sich dies bereits aus dem verfassungsrechtlichen Übermaßverbot ergeben ${ }^{41}$. So können zum einen bei fehlender Eignung die Zeugenpflichten objektiv nicht erfüllt werden. Zum anderen ist die Belastung des kindlichen Zeugen mit der Erscheinens- und Aussagepflicht - sofern nicht schon a priori ungeeignet - unverhältnismäßig, wenn die Partei andere Beweismöglichkeiten hat. Voraussetzung wäre demnach die vorherige Ausschöpfung aller anderen im konkreten Fall zur Verfügung stehenden Beweismittel ${ }^{42}$. Die Belastung der beweispflichtigen Partei beschränkt sich dann auf die Einschrän-

Meyer, S. 615 ff. Kritisch auch Wieczorek, § 286 Anm. C II a, der die Ablehnung wegen des geringen Beweiswertes einer erst nach längerer Zeit erstatten Aussage für unzulässig hält.

${ }^{37}$ Stein-Jonas-Schumann, § 373 Rn 1; Wieczorek, § 373 Anm. D I b 3; Bruns, Rn 87, der die Vernehmung eines Zeugen vom Hörensagen weitergehend auf die Fälle einschränken will, wenn kein Augenzeuge vorhanden ist.

${ }^{38} B G H Z$ 53, 245, 261; DRiZ 1974, 27, 28.

${ }^{39}$ Eine verstärkte Substantiierungslast ist auch bei einem Zeugenbeweis über innere Tatsachen anzunehmen. Der $B G H$ verlangt vom Beweisführer die Darlegung der Umstände, aufgrund derer der Zeuge die in sein Wissen gestellte Kenntnis erlangt haben soll, $B G H$, NJW 1983, 2034, 2035 (insoweit nicht veröffentlicht in BGHZ 87, 227 ff).

${ }^{40}$ So auch bereits Bosch, S. 45. Die dagegen geäußerten Bedenken von Orlowsky, S. $68 \mathrm{f}$ überzeugen nicht, zumal er selbst an anderer Stelle, S. 78, Unsicherheiten und Unwägbarkeiten aufgrund der geltenden Regelung einräumen muß.

${ }^{41}$ Zur Geltung des Übermaßverbotes im Zivilprozeß Stein-Jonas-Schumann, Einl. Rn 511; ebenda, Vor § 373 Rn 35 m.w.N..

${ }^{42}$ Ähnlich bei der Frage der Zulässigkeit von Glaubwürdigkeitsgutachten Stein-JonasSchumann, Vor § 373 Rn 39. Zum Strafprozeß wie hier auch Römer, RdJB 1971, $205,208$. 
kung der Wahl der Beweismittel, wenn die Vernehmung eines Minderjährigen nur bei voraussichtlich prozeßentscheidender Bedeutung der Aussage zugelassen wird.

Auch nach der Ausschöpfung der anderen Beweismittel kann der Beweisantrag noch verworfen werden, wenn eine Abwägung der Interessen der beweisführenden Partei und der des minderjährigen Zeugen zugunsten des letzteren ausfällt $t^{43}$. Der Minderjährigenschutz gewinnt besonderes Gewicht, wenn es sich um eine Ehe- und Familiensache handelt; hier ist der jugendliche Zeuge zusätzlich über die Zeugenpflichten als solche in seinen familiären und verwandschaftlichen Beziehungen betroffen. Sein Schutz ist hier nicht allein durch die Einräumung eines Zeugnisverweigerungsrechtes zu gewährleisten, sondern bei kindlichen Zeugen auch durch die Zurückweisung des Beweisantrages.

Die Entscheidung über die Zurückweisung der Beweisantretung wegen Benennung eines minderjährigen Zeugen steht grundsätzlich im pflichtgemäßen Ermessen des Gerichts ${ }^{44}$.

\footnotetext{
${ }^{43}$ So im Ergebnis auch Wieczorek, § 286 Anm. C IV a 3, der in Ehesachen Kinderaussagen "um der Kinder willen" nicht erlauben will; Schönke, FS für Rosenberg, S. 221. Nach Stern, DJZ 1910, 1002, 1003 sollten Kinderaussagen in Bagatellsachen (im Strafverfahren) durch die Einräumung eines besonderen Zeugnisverweigerungsrechtes verhindert werden. Der Schutzbedürftigkeit des Zeugen ist aber durch die Einräumung von Zeugnisverweigerungsrechten nicht ausreichend Rechnung getragen, da sie nur die Aussagepflicht, nicht aber die Erscheinenspflicht endgültig aufhebt. Auch bliebe denkbar, daß über die Berechtigung zur Zeugnisverweigerung ein Zwischenstreit geführt wird, wodurch der Schutz wieder in Frage gestellt wäre. In diesem Zusammenhang auch Stein-Jonas-Schumann, Vor § 373 Rn 33. Zum Zeugnisverweigerungsrecht des minderjährigen Zeugen unten § 6 .

${ }^{44}$ OLG Köln, Jur.Büro 1969, 645. Gegen die Annahme eines Ermessensspielraumes bei der Ablehnung von Beweisanträgen Teplitzky, JuS 1968, 71, 73 - die Bindung der Entscheidung an enge Voraussetzungen schließt jedoch einen Entscheidungs- und Beurteilungsspielraum des Richters nicht aus.
} 


\section{Glaubwürdigkeits- und Eignungsgutachten}

Der Ablehnung eines Zeugenbeweisantrages kann auch nicht durch einen gesonderten Antrag auf Einholung eines Sachverständigengutachtens über die Eignung des Zeugen begegnet werden. Zum einen ist kein Zeuge zur Mitwirkung, sondern allenfalls zur Duldung entsprechender Untersuchungen verpflichtet ${ }^{45}$, zum anderen handelte es sich bei einem derartigen Antrag um einen bloßen Beweisermittlungsantrag, dem nachzukommen das Gericht zumindest im Rahmen des Beibringungsgrundsatzes grundsätzlich nicht verpflichtet ist ${ }^{46}$.

Die Einwilligung in eine Begutachtung zur Feststellung der Zeugeneignung oder der Glaubwürdigkeit ist vom Zeugen zu erklären. Fehlt ihm die Fähigkeit, die Bedeutung seiner Einwilligung zu verstehen und danach zu handeln, entscheiden die gesetzlichen Vertreter kraft ihres Personensorgerechts ${ }^{47}$. Die Vorschriften des materiellen Rechts über die Geschäftsfähigkeit sind nicht anwendbar, weil es sich nicht um eine rechtsgeschäftliche, sondern um eine prozessuale Erklärung handelt; die Einwilligung ist deshalb wie der Verzicht auf ein Zeugnisverweigerungsrecht $\mathrm{zu}$ behandeln ${ }^{48}$.

\footnotetext{
${ }^{45}$ Kritisch Dippel, FS für Tröndle, S. 599, 621 ff; Löwe-Rosenberg-Dahs, § 81 c Rn 8 f; SteinJonas-Schumann, Vor $\$ 373$ Rn 37 ff, 40 Fn 66; Panhuysen, S. 81 ff zum Glaubwürdigkeitsgutachten; Musielak-Stadler, Rn 102 ff.

${ }^{46}$ Vgl. Goldschmidt, Der Prozeß, S. 445 Fn 2339; derselbe, ZPR, S. 136; Stein-Jonas-Leipold, $\S 284 \mathrm{Rn} 40$. Im Strafverfahren ist dagegen grundsätzlich die Einschränkung durch § 244 IV StPO zu beachten; dazu BGHSt. 3, 27, 29 f; 3, 52 ff; 13, 297, 300; BGH, NStZ 1981, 400; 85, 420; Kleinknecht/Meyer, §244 Rn 74. Der BGH vertritt die Auffassung, da 3 das erkennende Gericht im Regelfall selbst ausreichende Sachkenntnis besitzt, um die Glaubwürdigkeit zu beurteilen, nur in Ausnahmefällen wie etwa eigentümlicher Wesensart des Zeugen komme daher eine Untersuchung durch einen Sachverständigen in Betracht. Für den Fall der Zeugeneignung gilt grundsätzlich dasselbe, da es auch dort letztendlich um die Beweiskraft einer erstatteten oder noch zu erstattenden Aussage geht.

${ }^{47}$ Ebenso Löwe-Rosenberg-Dahs, $\$ 81$ c Rn 8, 43 f m.w.N. zum Strafverfahren.

${ }^{48} \mathrm{Vgl}$. unten $\S 6$. Im Strafprozeßrecht ergibt sich dies aus der Verweisung des $\S 81 \mathrm{c}$ III 2 StPO auf $\$ 52$ II 2 StPO. Da der Schutz des kindlichen Zeugen von der Verfahrensart unabhängig zu gewährleisten ist, besteht kein Grund, im Zivilprozeß die Einwilligung in die Begutachtung und den Verzicht auf ein Zeugnisverweigerungsrecht ungleich zu behandeln.
} 


\section{Rechtsbehelfe gegen die Entscheidung über einen Beweisantrag}

Soweit das Gericht einem Beweisantrag auf Vernehmung eines kindlichen Zeugen nachkommt und entsprechend den $\S 358 \mathrm{ff}$ ZPO einen Beweisbeschluß faßt, kommen gegen diesen Beschluß wegen $\S 355$ II ZPO keine Rechtsmittel in Betracht. Die Zivilprozeßordnung kennt keine selbständige Entscheidung über die Erheblichkeit einer Tatsache, die Beweisbedürftigkeit und die -last in Form eines Beweisinterlokutes ${ }^{49}$. Dies gilt auch für die Frage der Zulässigkeit und der Geeignetheit des Beweismittels selbst. Für den Zeugen ergibt sich dies darüberhinaus auch aus dem Umstand, daß seine Zeugenpflichten durch persönliche Eigenschaften im Grundsatz nicht aufgehoben werden und daher kein subjektives Recht auf eine bestimmte Ermessensausübung seitens des Gerichts angenommen werden kann.

Der Gegner des Beweisführers hat allerdings die Möglichkeit, einen Zwischenstreit über die Zulässigkeit der Beweiserhebung zu führen. Umgekehrt hat auch der Beweisführer diese Möglichkeit, wenn das Gericht seinen Antrag erkennbar nicht berücksichtigt. Das Gericht entscheidet in diesen Fällen durch Zwischenurteil gemäß § $303 \mathrm{ZPO}^{50}$. Das Zwischenurteil ist jedoch keine selbständige Entscheidung, sondern Teil des Endurteils; selbständige Rechtsbehelfe scheiden daher aus ${ }^{51}$. Beruht die Sache auf einem nach Ansicht der Parteien unvollständig aufgeklärten Sachverhalt, was die Verwertung der Aussage eines ungeeigneten Zeugen einschließen kann, stehen somit nur Berufung und Revision zur Verfügung ${ }^{52}$.

\footnotetext{
${ }^{49}$ Bruns, Rn 175; Mühl, ZZP 66, 165 ff; Rosenberg-Schwab, § 119 IV 2 c.

${ }^{50}$ Bruns, Rn 173 a; Goldschmidt, Der Prozeß, S. 451; Rosenberg-Schwab, § 58 III 1 a; SteinJonas-Schumann, § $359 \mathrm{Rn} 5$.

${ }^{51}$ Baumbach-Lauterbach-Hartmann, §359 Anm. 3; Stein-Jonas-Schumann, $\$ 359$ Rn 6. Anders bei Entscheidungen des beauftragten oder ersuchten Richters ( $\$ 366 \mathrm{ZPO}$ ), worauf in diesem Zusammenhang aber nicht einzugehen ist, da bei einer Beschwerde gegen dessen Entscheidung das Prozeßgericht entscheidet und insofern wieder das Gesagte gilt; Wieczorek, § 366 Anm. B II.

${ }^{52}$ LG Traunstein, FamRZ 1964, 97; Stein-Jonas-Leipold, § 284 Rn 52 zur Revision.
} 


\section{Ergebnis}

Zusammenfassend ist festzuhalten, daß grundsätzlich jeder Minderjährige als Zeuge vernommen werden kann, das Gericht nach pflichtgemäßem Ermessen aber ungeeignete Beweisanträge verwerfen darf. Bei Kindern unter sechs Jahren ist zu vermuten, daß die Eignung fehlt. Die Parteien haben gegenüber der Zurückweisung keine gesonderte Anfechtungsmöglichkeit, können aber die Vermutung fehlender Zeugeneignung bei Kindern im Vorschulalter widerlegen. Die Widerlegung setzt voraus, daß der Beweisantrag durch Angaben über besondere Umstände substantiiert wird, aufgrund derer erwartet werden kann, daß das Kind über die Beweistatsache doch eine verwertbare Aussage machen kann. 



\section{Die Stellung des Minderjährigen als Zeuge im Zivilprozeß}

\section{§ 4 Die Ladung des minderjährigen Zeugen}

\section{A. Zweck und Inhalt der Ladung}

Nach § 377 II ZPO muß ein Zeuge, dessen Vernehmung das Prozeßgericht durch einen entsprechenden Beweisbeschluß angeordnet hat, von Amts wegen geladen werden. Eine Ausnahme gilt für den gestellten, d.h. einen bereits von der Partei zur mündlichen Verhandlung mitgebrachten Zeugen ${ }^{1}$. Die Ladung erfolgt in der Regel formlos, sofern das Gericht nicht ausnahmsweise die förmliche Zustellung angeordnet hat ${ }^{2}$.

Die Ladung ist die Aufforderung an den Zeugen, am angegebenen Gerichtsort zu erscheinen; sie konkretisiert damit die vorher nur als allgemeine staatsbürgerliche Zeugenpflicht bestehende Erscheinenspflicht ${ }^{3}$ auf das Erscheinen zu einer bestimmten Zeit an einem bestimmten Ort. Die Ausübung eines Zeugnisverweigerungsrechtes suspendiert die Erscheinenspflicht nach Maßgabe des § 386 III ZPO zumindest vorläufigł.

\footnotetext{
${ }^{1}$ Baumbach-Lauterbach-Hartmann, $\$ 377$ Anm. 1 A; Stein-Jonas-Schumann, $\$ 377$ Rn 2. Bis 1976 war dies in § 357a ZPO gesetzlich geregelt; nach dieser Vorschrift konnten Zeugen und Sachverständige sofort vernommen werden, sofern sie zur Stelle waren oder unverzüglich gestellt werden konnten.

${ }^{2}$ Die förmliche Zustellung kann insbes. angeordnet werden, wenn Anhaltspunkte dafür vorliegen, der Zeuge werde sich seiner Erscheinenspflicht entziehen. Aufgrund des sicheren Nachweises des Zugangs der Ladung lassen sich in diesem Fall Ordnungsmittel zügiger verhängen, Baumbach-Lauterbach-Hartmann, § 377 Anm. 1 C.

${ }^{3}$ Bruns, Rn 184 a; näher zu den Zeugenpflichten unten $\$ 5 \mathrm{~A}$.

${ }^{4}$ Zum Zeugnisverweigerungsrecht unten $§ 6$.
} 
Der notwendige Inhalt der Ladung ergibt sich aus § 377 II ZPO. Danach muß die Ladung neben der Bezeichnung der Parteien, des Gerichts und des Gegenstands der Vernehmung auch einen Hinweis auf die Folgen eines unentschuldigten Fernbleibens enthalten. Der Zeuge soll dadurch in die Lage versetzt werden, sich auf seine Aussage vorzubereiten ${ }^{5}$. Darüberhinaus ist eine ordnungsgemäße Ladung Voraussetzung für die Ausübung staatlicher Zwangsmaßnahmen, mit denen das unentschuldigte Fernbleiben des Zeugen sanktioniert wird.

\section{B. Adressat der Ladung}

Bei einem Minderjährigen als Zeugen stellt sich die Frage, ob er selbst Adressat der Ladung ist oder ob statt seiner die Ladung an die gesetzlichen Vertreter zu richten ist.

\section{Der Minderjährige als Ladungsadressat}

Da der Minderjährige selbst Zeuge ist und das Gericht nicht notwendigerweise Kenntnis vom Alter des Zeugen hat ${ }^{6}$, ergibt sich schon daraus die Notwendigkeit, die Ladung an den Minderjährigen persönlich zu richten. Damit ist jedoch noch nicht entschieden, ob die Ladung des Minderjährigen allein bereits ausreicht ${ }^{7}$ oder ob die Beteiligung der Eltern geboten ist, wenn etwa der Geladene der Aufforderung gar nicht folgen kann. Dies wäre beispielsweise der Fall, wenn der minderjährige Zeuge, der noch im Vorschulalter sein kann, an einem nicht am Wohnort befindlichem Gericht vernommen werden soll.

\footnotetext{
${ }^{5}$ Baumbach-Lauterbach-Hartmann, $\$ 377$ Anm. 2; Stein-Jonas-Schumann, $\S 377$ Rn 9; Thomas-Putzo, § 377 Anm. 1.

${ }^{6}$ Dazu noch unter $\$ 4$ B III.

${ }^{7}$ So aber Schimmack, JW 1924, 1667, der de lege ferenda allenfalls erweiterte Möglichkeiten der Ersatzzustellung in Betracht ziehen will.
} 


\section{Ladung der gesetzlichen Vertreter}

\section{Die entsprechende Anwendung des $\$ 171 \mathrm{ZPO}$}

Die Durchführung der Ladung des minderjährigen Zeugen ist nicht unumstritten. So wird teilweise erwogen, § 171 I, III ZPO heranzuziehen. Nach dieser Vorschrift erfolgt die Zustellung bei einem nicht prozeßfähigen Adressaten an mindestens einen der gesetzlichen Vertreter. Die Vorschrift regelt unmittelbar nur die Zustellung an eine prozeßunfähige Partei, nicht aber an den Zeugen. Es käme daher allenfalls eine entsprechende Anwendung in Betracht ${ }^{8}$, da eine spezielle Regelung zum Zeugenrecht nicht besteht. Dagegen spricht jedoch zum einen, daß § $171 \mathrm{ZPO}$ gerade auf die Parteieigenschaft abstellt und für die förmliche Zustellung eine Regelung trifft, die bei der Zeugenladung nur ausnahmsweise anzuordnen ist. Darüberhinaus stellt § 171 ZPO als entscheidendes Kriterium auf die Prozeßfähigkeit $a b$ und entscheidet je nach Vorliegen dieser Eigenschaft über die Frage des Zustellungsadressaten. Auf die Prozeßfähigkeit kommt es aber bei der Ladung des Zeugen nicht an, weil auch der Prozeßunfähige zeugnisfähig und damit zeugnispflichtig ist ${ }^{9}$. Eine analoge Anwendung des $\S 171 \mathrm{ZPO}$ ist daher abzulehnen, zumal sie keine differenzierenden Lösungen zulassen würde, wie sie im Hinblick auf den durchaus unterschiedlichen Entwicklungsstand der Minderjährigen und der damit zusammenhängenden Rechtsposition geboten ist ${ }^{10}$.

Das gesetzliche Vertretungsrecht der Eltern nach § 1629 I BGB ist als Grundlage für die ausschließliche Zustellung der Ladung an die gesetzlichen Vertreter ebenfalls nicht geeignet, da es sich nicht um die Entgegennahme einer Willenserklärung handelt ${ }^{11}$.

${ }^{8}$ So offenbar Baumbach-Lauterbach-Hartmann, $\$ 377$ Anm. 1 C a.E.; auch Skupin, MDR 1965, 865, 866 nimmt an, daß die Erziehungsberechtigten Ladungsadressaten sind, weil sie auch für das Erscheinen des Kindes verantwortlich seien.

${ }^{9}$ Ebenso Wieczorek, $\S 380$ Anm. A I b 1.

${ }^{10}$ Gegen eine undifferenzierte Analogie auch Stein-Jonas-Schumann, § 377 Rn 3.

${ }^{11}$ Die Entgegennahme der Ladung, durch die die öffentlich-rechtliche Erscheinenspflicht konkretisiert wird, wäre eher als amtsähnliche Handlung anzusehen, unter der die Erfüllung einer öffentlich-rechtlichen Pflicht ohne Befugnis der Eltern zu einer eigenen Willensentschließung verstanden wird; dazu Soergel-Strätz, § $1629 \mathrm{Rn} 7$. 


\section{Einsichtsfähigkeit als bestimmendes Merkmal}

Als vermittelnde Lösung wird vorgeschlagen, die Frage des Adressaten der Ladung von der Einsichtsfähigkeit des zu ladenden Minderjährigen abhängig zu machen. Dieser Auffassung zufolge ist derjenige, der bereits in der Lage ist, Inhalt und Bedeutung der Ladung zu verstehen und ihr Folge zu leisten, stets selbst zu laden. In den übrigen Fällen, insbesondere bei sehr jungen Zeugen, die ohne Begleitung ihrer gesetzlichen Vertreter oder sonstiger Erwachsener nicht am Gerichtsort erscheinen können, hat die Ladung der gesetzlichen Vertreter, regelmäßig der Eltern, zu erfolgen ${ }^{12}$. Letzteres soll nach dieser Ansicht auch bei Zweifeln über die Einsichtsfähigkeit gelten; nach Vollendung des 14. Lebensjahres könne ein Zeuge in der Regel bereits selbst geladen werden ${ }^{13}$.

\section{Stellungnahme}

\section{Der Minderjährige als alleiniger Ladungsadressat}

Die Zustellung allein an die gesetzlichen Vertreter berücksichtigt die eigenständige prozessuale Rechtsstellung des Minderjährigen nicht hinreichend. Andererseits besteht die Gefahr, daß die Ladung des Minderjährigen selbst zumindest dann ihren Zweck verfehlt, wenn der Jugendlichen ihre Bedeutung nicht erkennen und der Aufforderung zum Erscheinen nachkommen kann. Dieser Konflikt scheint durch die zuletzt genannte, vermittelnde Ansicht sachgerecht gelöst zu werden, da sie eine Beteiligung der gesetzlichen Vertreter nur insoweit vorsieht, als sie zum Schutz des Minderjährigen erforderlich ist und im übrigen auf die selbständige Zeugenstellung des Minderjährigen abstellt.

Zweifelhaft ist jedoch die Praktikabilität dieses eher theoretischen Lösungsansatzes. Die Verknüpfung der Frage des richtigen Adressaten der Ladung und gegebenenfalls des Zustellungsempfängers mit der Einsichtsfä-

\footnotetext{
${ }^{12}$ So Stein-Jonas-Schumann, § $377 \mathrm{Rn}$ 3; ähnlich für die StPO Kleinknecht-Meyer, § $48 \mathrm{Rn} 7$.

${ }^{13}$ Stein-Jonas-Schumann, § 377 Rn 3.
} 
higkeit des Zeugen belastet bereits die Ladung mit praktisch schwierigen Abgrenzungsproblemen. Die Einsichtsfähigkeit kann stets nur individuell bestimmt werden, sieht man von den eindeutigen Bereichen am untersten und oberen Rand der Altersskala ab. Ohne unmittelbaren Eindruck des Gerichts von der Persönlichkeit des Zeugen ist dieses regelmäßig nicht in der Lage, die Entwicklung und die Reife des Zeugen zu beurteilen. Die Möglichkeit zur persönlichen Beurteilung durch das Gericht soll durch die Ladung aber erst vorbereitet werden ${ }^{14}$. Diese Schwierigkeiten werden noch verstärkt, wenn berücksichtigt wird, daß die Beweisantretung durch die Benennung des Zeugen einschließlich der ladungsfähigen Anschrift und des Beweisthemas geschieht. Sonstige Angaben und hier insbesondere des Alters des Zeugen sind nicht erforderlich ${ }^{15}$.

Die Schwierigkeiten können nicht gänzlich vermieden werden, doch sollte ein Weg gewählt werden, der den praktischen Bedürfnissen am weitesten entgegen kommt. Von daher erscheint es angemessen, die Ladung stets an den Minderjährigen selbst zu richten. Nur dieser unterliegt den Zeugenpflichten und ihm steht auch die Entscheidungsbefugnis zu, ob er den Pflichten Folge leisten will, wenn ein Zeugnisverweigerungsrecht besteht ${ }^{16}$.

\section{Die Benachrichtigung der gesetzlichen Vertreter}

Die Ladung des Zeugen ist um die Benachrichtigung der gesetzlichen Vertreter zu ergänzen, sobald dem Gericht bekannt wird, daß der Zeuge minderjährig ist. Dies erfolgt am zweckmäßigsten durch die Ladung zu Händen der gesetzlichen Vertreter ${ }^{17}$ oder einem nachträglichen Hinweis mit gesondertem Schreiben. Die Eltern sind im Rahmen ihres Personensorge-

\footnotetext{
${ }^{14}$ Ähnlich Löwe-Rosenberg-Gollwitzer, § 214 Rn 3; Skupin, MDR 1965, 865, 866.

${ }^{15}$ Anderes gilt nach hier vertretener Auffassung für Zeugen vor Vollendung des sechsten Lebensjahres; vgl. oben § 3 B III 2 b. Die Ablehnung eines Beweisantrages setzt allerdings voraus, daß das Gericht Anlaß zu Zweifeln an der Eignung des Zeugen hat. Diese Zweifel werden häufig auf einem unmittelbaren Eindruck von der Zeugenpersönlichkeit beruhen.

${ }^{16}$ Zur Ausübung eines Zeugnisverweigerungsrechts durch einen Minderjährigen unten § 6 B, C.

${ }^{17}$ Auch Baumbach-Lauterbach-Hartmann, §377 Anm. 1 C; Zöller-Stephan, §377 Rn 1, empfehlen die Ladung zu Händen der gesetzlichen Vertreter als zweckmäßig. Für die StPO KMR-Paulus, § $48 \mathrm{Rn} 13 ;$ Löwe-Rosenberg-Dahs, § $48 \mathrm{Rn} 10$.
} 
rechts nach § 1626 II BGB verpflichtet dafür Sorge zu tragen, daß der Minderjährige seinen Zeugenpflichten, hier also zunächst der Erscheinenspflicht, nachkommt ${ }^{18}$. Dazu müssen sie jedoch Kenntnis von der Ladung haben, was wiederum die Ladung zu Händen der gesetzlichen Vertreter voraussetzt.

Dies gilt grundsätzlich auch, wenn die Eltern selbst Partei des Prozesses und Beweisführer sind. Nur wenn im Einzelfall eine Interessenkollision zu befürchten ist, etwa wenn im Scheidungsverfahren der Eltern das gemeinsame Kind von dem Elternteil als Zeuge benannt wurde, der das Sorgerecht in tatsächlicher Weise nicht ausübt, kann die Bestellung eines Ergänzungspflegers bereits für die Ladung des Minderjährigen erforderlich werden ${ }^{19}$.

Voraussetzung ist, daß dem Gericht überhaupt Anhaltspunkte für die Minderjährigkeit des Zeugen vorliegen, was bei Zweifeln durch Rückfragen an die beweisführende Partei zu klären ist ${ }^{20}$.

${ }^{18}$ OLG Hamm, NJW 1965, 1613; Skupin, MDR 1965, 865, 867. Das OLG Saarbrücken, MDR 1974, 859, 860 = NJW 1974, 1959, 160 hält die Eltern für berechtigt, ihr Kind von der Hauptverhandlung fernzuhalten, wenn anderenfalls Entwicklungs- oder Erziehungsschäden drohen. Nur unter diesen Voraussetzungen stehe der Zeugenvernehmung ein Hindernis entgegen; kein Hindernis bestehe, wenn die Eltern mangels ausreichendem Grund gezwungen werden können, das Kind zur Verhandlung zu bringen. Das OLG Saarbrücken setzt sich mit der Frage, ob und wie die Eltern bei unberechtigter Weigerung gezwungen werden können, gerade nicht auseinander. Zur Mitwirkungspflicht der Eltern unten $\S 4 \mathrm{C}$ III.

${ }^{19}$ Soweit aufgrund der Interessenskollision Anla $\mathrm{zu}$ der Besorgnis besteht, ein Elternteil werde das Kind davon abhalten oder daran hindern, der Aufforderung der Ladung nachzukommen, sind die zur Ausübung des Zeugnisverweigerungsrechtes bei Beteiligung eines oder beider Elternteile dargelegten Grundsätze entsprechend heranzuziehen; dazu unten $\S 6 \mathrm{C}$ III, IV.

${ }^{20}$ Das ergibt sich aus $\S \S 139,278$ III ZPO und dem Rechtsgedanken des $\S 356$ ZPO. Ist das Gericht in diesem Punkt seiner Aufklärungspflicht nicht nachgekommen, tritt keine Präklusion ein; Stein-Jonas-Leipold, § 296 Rn 73; Thomas-Putzo, § 296 Anm. 1 g; Wieczorek, § 373 Anm. D II. 


\section{Hinweispflicht der Parteien}

Der Beweisführer ist aufgrund seiner Prozeßförderungspflicht ${ }^{21}$ gehalten, entsprechende Angaben von sich aus zu machen und gegebenfalls die Vermutung fehlender Zeugeneignung bei Kindern unter sechs Jahren ${ }^{22}$ zu widerlegen. Unterläßt die beweisführende Partei die erforderlichen Angaben und erreicht die Ladung deshalb ihren Zweck nicht, etwa weil das Gericht nur den Minderjährigen selbst geladen hat und die gesetzlichen Vertreter davon keine Kenntnis hatten oder sie sich nicht verpflichtet fühlten, das Kind zum Termin zu bringen, so geht dies prozessual zu Lasten der Partei. Voraussetzung ist allerdings, da $\beta$ der Beweisführer die erforderlichen Umstände, aus denen sich seine besondere Erklärungspflicht ergibt, selbst kannte oder kennen mußte. Nur bei schuldhaften Verstößen gegen prozessuale Pflichten kommen entsprechende Sanktionen in Betracht ${ }^{23}$. Dabei ist zu unterscheiden, ob der Zeuge bereits verspätet benannt wurde, oder ob er zwar rechtzeitig, aber im oben genannten Sinne unvollständig benannt wurde. Im ersten Fall kann der Beweisantrag nach Maßgabe des $\S 296$ II ZPO als präkludiert zurückgewiesen werden ${ }^{24}$. Anderenfalls muß dem Beweisführer seitens des Gerichts eine Frist gesetzt werden, binnen derer er sich über die Unvollständigkeit zu erklären hat ${ }^{25}$; nach erfolglosem Ablauf der Frist kann der Beweisantrag gemäß § 356 ZPO verworfen werden.

Der Fall des unterbliebenen Hinweises auf das jugendliche Alter durch die Partei ist daher gleich dem Fehlen einer ladungsfähigen Anschrift oder

\footnotetext{
${ }^{21}$ Sie ergibt sich auch aus $\$ 282$ I ZPO und gebietet den Parteien, ihre Beweismittel frühzeitig und umfassend anzubieten, Stein-Jonas-Leipold, § $282 \mathrm{Rn} 19$. Die Parteien entsprechen der Prozeßförderungspflicht nicht durch unvollständige Beweisanträge; ähnlich LG Frankfurt, NJW-RR 1986, 143. Zum Verhältnis von Prozeßförderungspflicht und Präklusion auch Franke, NJW 1986, 3049, 3053; Kallweit, S. 24 ff. Kritisch zu dem Begriff der Prozeßförderungspflicht Leipold, ZZP 93, $237 \mathrm{ff}$.

${ }^{22} \mathrm{Vgl}$. oben § 3 B III 2 c.

${ }^{23}$ So setzt die Präklusion verspäteten Vorbringens als Einschränkung des rechtlichen Gehörs stets die schuldhafte Verletzung prozessualer Obliegenheiten voraus; BGH, NJW 1989, 227 f; Franke, NJW 1986, 3049 ff m.w.N.; Kallweit, S. 66 ff; Leipold, ZZP 93, 237 ff; Schwab/ Gottwald, Verfassung und Zivilprozeß, S. 61 f;

${ }^{24}$ Stein-Jonas-Leipold, § $296 \mathrm{Rn} 45 ;$ Stein-Jonas-Schumann, § $356 \mathrm{Rn} 1$.

${ }^{25}$ Aus Gründen der Rechtsklarheit über den Lauf der Frist muß die Fristsetzung förmlich zugestellt werden, $B G H$, NJW 1989, 227 f; dazu auch $B V e r f G$, NJW 1984, 1026
} 
der Beweisantretung durch den Zeugen "N.N." ${ }^{26}$ zu behandeln. Hier wie dort kann der Zeuge wegen eines Unterlassens der beweisführenden Partei nicht zum Termin geladen werden, was die Gefahr der Verzögerung des Verfahrens schafft.

\section{Ordnungsmittel bei Nichterscheinen}

\section{Darstellung und Verwirkung der Ordnungsmittel}

Erscheint der Zeuge trotz ordnungsgemäßer Ladung nicht, ist er nach $\S 380 \mathrm{ZPO}$ von Amts wegen ${ }^{27}$ zur Tragung der durch sein Ausbleiben verursachten Kosten zu verurteilen und mit Ordnungsmitteln zu belegen.

Als Ordnungsmittel ist in erster Linie die Verhängung eines Ordnungsgeldes vorgesehen, das gemäß Art. 6 EGStGB zwischen fünf und eintausend DM liegen kann. Zugleich ist ersatzweise für den Fall, daß das Ordnungsgeld nicht beigetrieben werden kann, eine Ordnungshaft zwischen einem Tag und sechs Wochen anzudrohen ${ }^{28}$. Bei wiederholtem Ausbleiben sieht $\S 380$ II ZPO darüberhinaus die zwangsweise Vorführung des Zeugen vor. Die Verurteilung in die durch das Ausbleiben verursachten Kosten steht neben der Verhängung der genannten Ordnungsmittel.

Neben einer formal ordnungsgemäßen Ladung setzt die Verhängung von Zwangsmaßnahmen voraus, daß die Ladung statthaft war; dies ist insbesondere dann nicht der Fall, wenn der Zeuge bereits von einem Zeugnisverweigerungsrecht Gebrauch gemacht hat ${ }^{29}$. Dagegen kann die fehlende Eignung

\footnotetext{
${ }^{26}$ Zum Zeugen "N.N." und der nachträglichen Bezeichnung von Zeugen Rixecker, NJW 1984, 2135 f; Baumbach-Lauterbach-Hartmann, § 356 Anm. 1 A b; Stein-Jonas-Leipold, § 296 Rn 45; Thomas-Putzo, § 296 Anm. 2 b; BGH, NJW 1989, $227 \mathrm{f}$.

${ }^{27}$ Bergerfurth, JZ 1971, 84, 86; Baumbach-Lauterbach-Hartmann, § 380 Anm. 1 C a; ZöllerStephan, § $380 \mathrm{Rn} 3$.

${ }^{28}$ Art. 7 EGStGB; Baumbach-Lauterbach-Hartmann, $\$ 380$ Anm. 1 C c.

${ }^{29} \mathrm{Vgl}$. § 386 III ZPO, wonach der Zeuge nach schriftlich oder zu Protokoll der Geschäftsstelle erklärter Zeugnisverweigerung nicht zum Termin zu erscheinen braucht. Ferner Baumbach-Lauterbach-Hartmann, §380 Anm. 1 A; Wieczorek, $\S 380$ Anm. A II c.
} 
des Zeugen allein nicht schon zur Unstatthaftigkeit der Ladung führen, da das Gericht durch die Ladung gerade zu erkennen gegeben hat, daß ein Fall fehlender Eignung nicht vorliegt. Anderenfalls wäre bereits der Beweisantrag verworfen worden und die Ladung unterblieben. Bereits verhängte Maßnahmen sind nach Maßgabe des $\S 381$ ZPO wieder zurückzunehmen, wenn der Zeuge sein Fernbleiben ausreichend entschuldigt.

\section{Die Verhängung von Ordnungsmitteln gegen einen Minderjährigen}

\section{Ordnungsmittel und Kostentragung}

Der Minderjährige ist stets selbst Adressat der Ladung und der damit verbundenen Aufforderung, vor Gericht zu erscheinen. Die Erscheinenspflicht ist höchstpersönlicher Natur ${ }^{30}$, so daß zunächst an die Verhängung von Ordnungsmitteln gegen den Jugendlichen selbst zu denken ist.

Die Verhängung von Ordnungsmitteln gegen Minderjährige wurde nach einer früher vertretenen Auffassung ohne Einschränkung für zulässig erachtet. Begründet wurde dies damit, daß es sich bei den Folgen unentschuldigten Fehlens nicht um eine Strafe für kriminelles Unrecht, sondern lediglich um eine Ordnungsstrafe handele ${ }^{31}$. Dementsprechend konnte auch ein strafunmündiger Minderjähriger mit einer Ordnungsstrafe belegt werden, es sei denn, im Einzelfall entfiel der Schuldvorwurf, etwa weil die Eltern das Kind am Erscheinen vor Gericht hinderten ${ }^{32}$.

Gegen diese Meinung wurde eingewendet, die Verhängung eines Ordnungsgeldes ziehe wegen der regelmäßig vorliegenden Vermögenslosigkeit des Kindes den Vollzug der Ersatzfreiheitsstrafe nach sich, was dem Willen des Gesetzgebers widerspreche ${ }^{33}$. Dabei ist aber $\mathrm{zu}$ beachten, daß es sich nicht nur um ein Problem der Vollstreckbarkeit handelt, sondern um die Frage der Zulässigkeit der Verhängung von Ordnungsmitteln überhaupt.

\footnotetext{
${ }^{30} \mathrm{Zu}$ den Zeugenpflichten näher unten $\S 5 \mathrm{~A}$.

${ }^{31} R G$ GA 59, 131 f; Renner, Das Recht 1917, 378, 379; OLG Köln, NJW 1978, 2515, 2516.

${ }^{32}$ So etwa aus neuerer Zeit OLG Hamm, NJW 1965, 1613.

${ }^{33}$ Skupin, MDR 1965, 865, 866; LG Bremen, NJW 1970, 1429 f.

4 Findeisen
} 
Richtigerweise kann die Unterscheidung zwischen Kriminalstrafe und Ordnungsunrecht nicht mehr als allein maßgeblich angesehen werden. Auch bei den in $\S 380$ ZPO vorgesehenen Maßnahmen handelt es sich um eine Sanktion repressiven Charakters für pflichtwidriges Verhalten ${ }^{34}$. Die Sanktion setzt regelmäßig Vorwerfbarkeit und diese wiederum Schuldfähigkeit voraus $^{35}$. Das Verschulden als Voraussetzung für die Verurteilung in Ordnungsmittel ergibt sich auch aus $\S 381 \mathrm{ZPO}$, wonach bei genügender Ent schuldigung des Zeugen die Pflichtwidrigkeit entfällt. Bei einem minderjährigen Zeugen ist die Schuldfähigkeit positiv festzustellen36.

Der Gesetzgeber hat im Bereich des Strafrechts und des Jugendstrafrechts sowie des Ordnungswidrigkeitenrechts eine Vermutung normiert, wonach Jugendliche unter 14 Jahren nicht schuldfähig sind. Obwohl die Zivilprozeßordnung keine eigene Regelung dieser Frage enthält, entspricht es allgemeiner Überzeugung, die Schuldfähigkeit wegen des strafähnlichen Charakters der Ordnungsmittel ebenfalls dieser Altersgrenze zu unterwerfen $^{37}$. Die Deliktsfähigkeit nach Bürgerlichen Recht ist demgegenüber kein geeigneter Ansatzpunkt, weil § 828 BGB nur die zivilrechtliche Haftung und den Schadensausgleich gegenüber Dritten betrifft, jedoch keinen Strafcharakter besitzt ${ }^{38}$.

\footnotetext{
${ }^{34}$ So auch Schneider, NJW 1979, 987, 988 unter Hinweis auf die "Parallelwertung in der Laiensphäre", die in beiden Fällen eine Bestrafung erblicke. Brehm, NJW 1976, 1730, 1731 stellt (bei Ordnungsmitteln nach $\S 890 \mathrm{ZPO}$ ) auf dieselbe Wirkung beim Betroffenen ab. Ebenso OLG Hamm, NJW-RR 1987, 765, 766.

${ }^{35}$ Ähnlich zu den Ordnungsmitteln nach $\S 890$ ZPO auch BVerfGE 20, 223, 331; 58, 159, 162, wonach der Grundsatz "nulla poena sine culpa" Verfassungsrang genießt und für alle Strafarten mit Sühnecharakter gilt. Ferner Brehm, NJW 1974, 1730 f. Zur Abgrenzung von Ordnungs- und Zwangsmitteln unten $\$ 5 \mathrm{C}$.

${ }^{36}$ Baumbach-Lauterbach-Hartmann, $\$ 380$ Anm. 1 C c.

${ }^{37}$ Baumbach-Lauterbach-Hartmann, ebenda; Stein-Jonas-Schumann, §380 Rn 15; ZöllerStephan, $\S 380$ Rn 7; Musielak-Stadler, Rn 76. Wieczorek, §380 Anm. A I und A II c 2, hält es unter Hinweis auf $\S 107$ BGB für bedenklich, Minderjährige über sieben Jahre mit Ordnungsgeld oder Ersatzordnungshaft zu belegen. Die Bezugnahme auf die Vorschriften des materiellen Rechts über die Geschäftsfähigkeit ist jedoch abzulehnen, da $\S 380$ ZPO keine rechtsgeschäftliche oder rechtsgeschäftsähnliche Haftung betrifft. Ebenso im Strafprozeß KleinknechtMeyer, §51 Rn 15; Löwe-Rosenberg-Dahs, § 51 Rn 2; Skupin, MDR 1965, 865, 866 f; LG Bremen, NJW 1970, 1430 zur StPO. Anders aber bei den Kosten.

${ }^{38}$ MünchKomm-Mertens, Vor $\S \S 823-853$ Rn 41; Erman-Drees ${ }^{7}$, Vor $\S 823$ Rn $48 \mathrm{ff}$. Einschränkend Erman-Schiemann ${ }^{8}$, ebenda Rn 12 ff.
} 
Als Ergebnis ist damit festzuhalten, daß gegen einen schuldunfähigen Minderjährigen, mithin vor Vollendung des 14. Lebensjahres, keine Ordnungsmittel verhängt werden können. Nach Vollendung des 14. Lebensjahres kommen Ordnungsgeld und Ordnungshaft in Betracht, wenn der Zeuge im Einzelfall die notwendige Verstandesreife hatte, die Bedeutung der Ladung und die Folgen seines Ausbleibens zu verstehen. Fehlt es hieran, kann auch dem bereits älteren Minderjährigen kein Schuldvorwurf gemacht werden.

Nach § 381 ZPO entfällt bei hinreichender Entschuldigung auch die Verpflichtung, die durch das Ausbleiben verursachten Kosten zu tragen ${ }^{39}$. Auch insoweit kommt es daher auf Verschulden an. Die Schuldfähigkeit ist als Fähigkeit zu verstehen, die Folgen eigenen Verhaltens vorherzusehen und entsprechend dieser Einsicht zu handeln. Sie ist bezüglich der Ordnungsmittel und der Kostentragungspflicht vom Gericht von Amts wegen getrennt zu prüfen. Es ist nicht auszuschließen, daß der Minderjährige zwar eine Bestrafung, nicht aber die Verurteilung in die Kosten als Folge seines Ausbleibens verstehen konnte ${ }^{40}$.

Da die Auferlegung der Kosten keine Sanktion für pflichtwidriges Verhalten darstellt, sondern ein Ausgleich für die vom Zeugen verursachten Mehrkosten des Verfahrens, die sonst den Parteien zur Last fielen, ist m.E. daran zu denken, die Verantwortung des Minderjährigen insoweit nicht entsprechend § 19 StGB zu regeln, sondern analog der deliktischen Haftung nach Bürgerlichem Recht. Nach § 828 II BGB kommt die Auferlegung der Kosten damit bereits mit Vollendung des 7. Lebensjahres in Betracht, wenn der Minderjährige zur Zeit der Ladung die Fähigkeit zu der Einsicht hatte, daß sein Verhalten zusätzliche Kosten verursacht und ihm auch im übrigen ein Schuldvorwurf im Sinne des $\S 828$ II BGB gemacht werden kann.

\footnotetext{
${ }^{39} \mathrm{Zu}$ den Kosten gehören z.B. Reisekosten der Parteien und gerichtliche Kosten zur Überprüfung eines Entschuldigungsgrundes; Bergerfurth, JZ 1971, 84, 86; Wieczorek, § 380 Anm. B II a 1.
}

${ }^{40}$ Stein-Jonas-Schumann, § $380 \mathrm{Rn} 15$. 


\section{Die zwangsweise Vorführung}

Zu klären bleibt, ob trotz der im Einzelfall unzulässigen Verhängung von Ordnungsmitteln, wenigstens die zwangsweise Vorführung des Zeugen gemäß § 380 II ZPO angeordnet werden kann. Bedeutung gewinnt dies insbesondere, wenn die gesetzlichen Vertreter bereits zu erkennen gegeben haben, daß sie das Kind nicht zum Termin bringen werden.

Für den Strafprozeß, der in § 51 StPO eine ähnliche Regelung enthält, wird die zwangsweise Vorführung eines Schuldunfähigen für zulässig erachtet. Die Vorführung stelle keine Ahndung eines Gesetzesverstoßes dar, sondern diene lediglich dazu, das Erscheinen des Zeugen sicherzustellen ${ }^{41}$.

Dagegen spricht jedoch, daß auch die sonstigen Ordnungsmittel das Ziel verfolgen, den Zeugen zum Erscheinen anzuhalten. Darüberhinaus ist nach $\S 380$ II ZPO die zwangsweise Vorführung anders als im Strafprozeß erst nach erfolgloser Verhängung eines Ordnungsmittels zulässig. Bei einem Minderjährigen, der noch nicht schuldfähig ist, könnte die Vorführung daher nur angeordnet werden, wenn zuvor ein offensichtlich unzulässiges Ordnungsmittel verhängt würde. Die besondere Eingriffsintensität gerade der zwangsweisen und gegen den Willen der Eltern durchgeführten Vorführung $^{22}$ erfordert den besonderen Schutz des Minderjährigen. Dem kann nur dadurch Rechnung getragen werden, die Einsichtsfähigkeit auch gerade im Hinblick auf diese Folge des Ausbleibens als Vorausetzung zu fordern. Die Vorführung ist daher nur rechtmäßig, wenn der Zeuge im strafrechtlichen Sinne schuldfähig ist und hinreichende Verstandesreife zum Zcitpunkt des Vernehmungstermins hatte.

\footnotetext{
${ }^{41}$ Löwe-Rosenberg-Duhs, § 51 Rn 2; Kleinknecht-Meyer, § 51 Rn 20; Günter, JA 1979, 428. OLG Düsseldorf, FamRZ 1973, 547 hält die zwangsweise Vorführung einer nach damaligem Recht noch Minderjährigen für zulässig; zu dieser Entscheidung auch unten § $5 \mathrm{~A} \mathrm{I}$.

${ }^{42}$ Skupin, MDR 1965, 865, 866 weist darauf hin, daß für das Kind regelmäßig die Entscheidung der Eltern maßgebend sein wird, ob es zur Vernehmung erscheint oder nicht, so daß die zwangsweise Vorführung gegen den Willen der Eltern oder sogar gegen deren Widerstand erfolgen müßte.
} 


\section{Ordnungsmittel gegen die gesetzlichen Vertreter}

Die gesetzlichen Vertreter sind im Rahmen ihres Sorgerechts nach $\S 1626$ II BGB verpflichtet, den Minderjährigen zum Erscheinen vor Gericht zu veranlassen und ihn gegebenenfalls selbst zum Termin zu bringen ${ }^{43}$. Dies gilt auch, wenn die gesetzlichen Vertreter selbst nicht als Zeugen oder Partei geladen sind, sondern nur aus Zweckmäßigkeitserwägungen neben dem Zeugen über dessen Ladung in Kenntnis gesetzt wurden ${ }^{44}$.

Soweit der Minderjährige nicht mit Ordnungsmitteln belegt werden kann, weil ihm noch die notwendige Einsichtsfähigkeit fehlt, wurde vereinzelt erwogen, statt dessen die gesetzlichen Vertreter Zwangsmaßnahmen zu unterwerfen, wenn sie schuldhaft ihre Pflichten verletzen ${ }^{45}$. Da $§ 380$ ZPO jedoch nur Sanktionen gegen den Zeugen vorsieht, fehlt für eine Bestrafung der gesetzlichen Vertreter eine gesetzliche Grundlage ${ }^{46}$.

Auch die Verpflichtung der gesetzlichen Vertreter, im Rahmen ihres Personensorgerechts das Kind zum Erscheinen anzuhalten, kann keine Ordnungsmittel rechtfertigen ${ }^{47}$. Es handelt sich nicht um eine prozessuale Pflicht gegenüber den Parteien oder dem Gericht, sondern nur um eine Verpflichtung gegenüber dem Kind. Daher kann die Einhaltung dieser Pflicht nicht mit Mitteln erzwungen werden, die gerade für die prozessualen Zeugenpflichten vorgesehen sind.

Das OLG Saarbrücken hat in einem Strafverfahren infolge der Weigerung der Sorgeberechtigten, den kindlichen Zeugen zum Termin mitzubringen, ein nicht zu beseitigendes Hindernis im Sinne des $§ 251$ I Nr. 2 StPO angenommen ${ }^{48}$. Das Gericht ist daher im Ergebnis ebenfalls von der Unzulässig-

\footnotetext{
${ }^{43}$ Skupin, MDR 1965, 865, 867; OLG Saarbrücken, MDR 1974, 859, 860 = NJW 1974, 1959 f.

${ }^{44}$ Zum Adressaten der Ladung oben $\S 4 \mathrm{~B}$.

${ }^{45}$ So z.B. Bosch, S. 57; Grundmann, S. 98 jedoch ohne Begründung.

${ }^{46}$ OLG Hamm, NJW 1965, 1613; Baumbach-Lauterbach-Hartmann, § 380 Anm. 1 C c; SteinJonas-Schumann, § $380 \mathrm{Rn} 15 ;$ Zöller-Stephan, § $380 \mathrm{Rn} 7$. Zur StPO Kleinknecht/Meyer, § 51 Rn 1; Löwe-Rosenberg-Dahs, § 51 Rn 4; Skupin, MDR 1965, 865, 867;

${ }^{47}$ OLG Hamm, NJW 1965, 1613.

48OLG Saarbrücken, MDR 1974, 859, 860 = NJW 1974, 1959, 1960; dazu auch Kleinknecht / Meyer, § $251 \mathrm{Rn} 7$.
} 
keit des Zeugenzwangs gegen die gesetzlichen Vertreter ausgegangen, denn nur dann kann ein nicht zu beseitigendes Hindernis angenommen werden.

Unabhängig von Sanktionen nach § 380 ZPO soll aber die Möglichkeit bestehen, nach § 1666 BGB vormundschaftsgerichtliche Maßnahmen bis hin zu einer Entziehung des Aufenthaltsbestimmungsrechts anzuregen ${ }^{49}$. Skupin begründet dies damit, daß die grundlose oder sachlich nicht gerechtfertigte Weigerung der Eltern, das Kind als Zeuge vernehmen zu lassen, häufig auf eine "staatsfeindliche Beeinflussung" des Kindes schließen lasse ${ }^{50}$.

Dagegen ist jedoch einzuwenden, daß $\S 1666$ BGB dem Schutz des Kindes vor mißbräuchlicher Sorgerechtsausübung und Versagen der Eltern dient $^{51}$. Soweit diese Voraussetzungen im Einzelfall gegeben sind, mag ein Einschreiten des Vormundschaftsgerichtes gerechtfertigt sein. Es kann aber nicht schon immer dann ein Fall des Mißbrauchs oder des Versagens angenommen werden, wenn die Eltern nicht für das Erscheinen des geladenen Kindes sorgen ${ }^{52}$. Staatliche Eingriffe in das Erziehungsrecht der Eltern, auch in Gestalt vormundschaftsgerichtlicher Maßnahmen, sind ferner nur zulässig, um Schäden für das Kind abzuwenden ${ }^{53}$. Der Einsatz des Vormundschaftsgerichts zur mittelbaren Sanktionierung der Eltern wegen Verletzung ihrer Mitwirkungspflichten ist daher abzulehnen.

Die Erscheinenspflicht des strafunmündigen Minderjährigen ist somit nicht strafbewehrt, insbesondere kann das Erscheinen vor Gericht nicht erzwungen werden ${ }^{54}$. Es handelt sich um eine "lex imperfecta", was jedoch als Folge des Minderjährigenschutzes von den Betroffenen, insbesondere der beweisführenden Partei hinzunehmen ist.

\footnotetext{
${ }^{49}$ Skupin, MDR 1965, 865, 868.

${ }^{50}$ Skupin, ebenda. Zur Aufsicht des Vormundschaftsgerichtes nach §1666 BGB allgemein Beitzke, § $31 \mathrm{I}$; Gernhuber, § 49 VIII 3 bis 5 und unten $\S 6$ C II 2.

${ }^{51}$ Soergel-Strätz, § 1666 Rn 16 ff; MünchKomm-Hinz, § 1666 Rn 20 ff.

${ }^{52}$ Zum Verhältnis zwischen Erscheinenspflicht und Aufenthaltbestimmungsrecht näher unten $\S 5 \mathrm{~A}$ I.

${ }^{53}$ BVerfGE 24, 119, 144 f. Erman-Michalski, $\$ 1666$ Rn 1 unter Hinweis, darauf, daß die Vorschrift dem Schutz des Kindes, nicht der Besserung der Eltern diene.

${ }^{54} \mathrm{Zu}$ den Sanktionen bei Verletzung der sonstigen Zeugenpflichten, insbesondere $\S 390$ $\mathrm{ZPO}$ unten $\S 5 \mathrm{C}$.
} 


\section{Nachträgliche Entschuldigung gemäß § 381 ZPO}

Die Verhängung von Ordnungsmitteln unterbleibt, bereits verhängte Ordnungsmittel sind wieder aufzuheben, wenn der Zeuge sein Fernbleiben genügend entschuldigt und die Gründe glaubhaft macht ${ }^{55}$.

Da gegen den schuldunfähigen Zeugen die Verhängung von Ordnungsmitteln stets unzulässig ist, kommt es auf seine Gründe für das Fernbleiben regelmäßig nicht an. Wurden dennoch Ordnungsmittel verhängt, sind sie von Amts wegen ${ }^{56}$ wieder aufzuheben. Entsprechendes gilt für den bereits strafmündigen Zeugen, der nicht die erforderliche Verstandesreife hatte, die Ladung zu verstehen. Er ist auch dann als entschuldigt anzusehen, wenn die gesetzlichen Vertreter die Möglichkeit der Aufklärung hatten, sie davon aber keinen Gebrauch machten. Anderenfalls würde der Minderjährige aufgrund einer Sorgerechtsverletzung der Eltern mit Ordnungsmitteln belegt.

Der strafmündige Zeuge mit ausreichender Verstandesreife ist einem Volljährigen gleichzustellen, so daß insoweit keine Besonderheiten auftreten $^{57}$. Da das Gericht die Entschuldigungsgründe von Amts wegen zu beachten hat, wenn Umstände für deren Vorliegen gegeben sind und hier auch solche Umstände zu berücksichtigen sind, die dem Gericht von dritter Seite zur Kenntnis gebracht werden ${ }^{58}$, kommt es auf die Vertretungsmacht der Eltern oder ihre Verhinderung im Einzelfall nicht an.

\footnotetext{
${ }^{55}$ Neben der Entschuldigung hat der Betroffene auch die Möglichkeit der Beschwerde nach $\S 380$ III ZPO; Stein-Jonas-Schumann, § 381 Rn 14; Zöller-Stephan, § 381 Rn 4. Dazu unten $\S 10 \mathrm{~B}$.

${ }^{56}$ Stein-Jonas-Schumann, § $381 \mathrm{Rn} 3$.

${ }^{57}$ Die Gleichstellung gilt allerdings auch hinsichtlich der Einlegung der Beschwerde nach $\S$ 380 III ZPO; zur Beschwerde allgemein unten § 10 B II 1.

${ }^{58}$ Stein-Jonas-Schumann, § $381 \mathrm{Rn} 3$.
} 


\section{Beschwerde gegen die Verhängung von Ordnungsmitteln}

Nach § 380 III ZPO findet gegen Gerichtsbeschlüsse, durch die ein Zeuge in Ordnungsgeld, -haft oder die Kosten verurteilt wird, die einfache Beschwerde statt. Im Zusammenhang mit dem Zeugenbeweis sind Beschwerdemöglichkeiten ferner in § 387 III, § 390 III ZPO vorgesehen. Unterschiede liegen hier jeweils nur im Hinblick auf den Beschwerdegegenstand vor; die Rechtsstellung des Minderjährigen im Beschwerdeverfahren soll daher im Zusammenhang mit dem Zwischenstreit nach §§ 387, 388 ZPO dargestellt werden ${ }^{59}$.

\section{Ergebnis}

Adressat der Zeugenladung ist regelmäßig der Zeuge selbst, also auch der minderjährige Zeuge. Nur wenn dem Gericht Anhaltspunkte für die Minderjährigkeit des Zeugen überhaupt vorliegen, erfolgt die Ladung zu Händen der gesetzlichen Vertreter oder durch gesonderte Mitteilung, wenn die Minderjährigkeit dem Gericht erst nach der Ladung, aber vor der Vernehmung bekannt wird. Nur so ist sichergestellt, daß die Ladung ihren Zweck erreichen kann. Auf die Einsichtsfähigkeit des Zeugen hinsichtlich Inhalt und Bedeutung der Ladung braucht das Gericht nicht zu achten.

Erreicht die Ladung ihren Zweck nicht, weil die beweisführende Partei es unterlassen hat, auf das Alter des Zeugen hinzuweisen, geht dies zu ihren Lasten.

Ordnungsmittel können nur dem Jugendlichen auferlegt werden, der zur Zeit der Ladung bereits das 14. Lebensjahr vollendet hat und darüberhinaus die geistige Reife besitzt, seine Erscheinenspflicht und gegebenenfalls die jeweiligen Folgen unentschuldigten Fernbleibens zu begreifen und entsprechend zu handeln. Dies gilt im besonderen Maße für die zwangsweise Vor-

\footnotetext{
${ }^{59}$ Unter $\$ 10$.
} 
führung. Die Kostentragungspflicht entsteht wegen der fehlenden Straffunktion bereits mit Deliktsfähigkeit analog § 828 II BGB.

Die gesetzlichen Vertreter sind aufgrund ihres Sorgerechts verpflichtet, das Kind zur Verhandlung zu bringen oder sonst sein Erscheinen zu gewährleisten. Verstöße der Eltern hiergegen können jedoch nicht nach § 380 ZPO geahndet werden. 



\section{§ 5 Die Zeugnispflichten}

\section{A. Darstellung}

Die Zeugnispflicht ist ein öffentlich-rechtliches Gebot, das sich aus der Pflicht zum Erscheinen vor Gericht, zur Erstattung einer wahrheitsgemäßen und vollständigen Aussage sowie gegebenenfalls zur Eidesleistung zusammensetz ${ }^{1}$. Sie ist nicht ausdrücklich geregelt, wird aber vom Gesetz vorausgesetzt. Dies ergibt sich schon aus der Existenz gesetzlich geregelter Zeugnisverweigerungsrechte und der Möglichkeit, bei Verstößen gegen Zeugenpflichten Ordnungs- und Zwangsmittel zu verhängen ${ }^{2}$. Sie dienen dem Schutz der Rechtsordnung und sind darüberhinaus höchstpersönlicher Natur $^{3}$. Nach dem Gesagten treffen diese Pflichten jedermann, also auch den Minderjährigen, sofern er nur ordnungsgemäß geladen wurde und nicht von der deutschen Gerichtsbarkeit befreit ist ${ }^{4}$.

${ }^{1}$ RGZ 20, 378 ff; Baumbach-Lauterbach-Hartmann, Übers. § 373 Anm. 3 A; Bruns, Rn 184; Goldschmidt, Der ProzeB, S. 109; Niese, S. 142 f; R. Schmidt, S. 478 f; Stein-Jonas-Schumann, Vor § $373 \mathrm{Rn} 31$; ebenda, Einl. Rn 236. Der Hinweis, es handele sich um eine staatsbürgerliche Pflicht ist dagegen ungenau und führt nicht darüber hinweg, da $\beta$ es sich um eine prozessuale Pflicht handelt, weil sie stets nur innerhalb eines Prozesses besteht; dazu Lent, ZZP 67, 344, 353 f.

${ }^{2} \mathrm{Vgl}$. dazu Gebhardt, S. 45.

${ }^{3}$ BGHZ 48, 324; Rosenberg-Schwab, § 123 III; Stein-Jonas-Schumann, Vor § 373 Rn 17.

${ }^{4}$ Baumbach-Lauterbach-Hartmann, Übers. § 373 Anm 3 A; Rosenberg-Schwab, § 123 III. 


\section{Die Erscheinenspflicht und das Aufenthaltsbestimmungsrecht der Sorgeberechtigten}

Die Erscheinenspflicht besagt, daß der Zeuge an dem in der Ladung bestimmten Termin vor Gericht anwesend zu sein hat. Bei einem minderjährigen Zeugen stellt sich in diesem Zusammenhang die Frage, in welchem Verhältnis die Pflicht zum Erscheinen an einem bestimmten Ort und einer bestimmten Zeit zu dem in $\S 1631$ I BGB geregelten Aufenthaltsbestimmungsrecht der gesetzlichen Vertreter steht, mit anderen Worten, ob die gesetzlichen Vertreter ihre Zustimmung zum Erscheinen des Minderjährigen erteilen müssen bzw. sie versagen können ${ }^{5}$. In einem vergleichbaren Fall, der ein Strafverfahren und hier die Teilnahme der nach damaligem Recht mit 19 Jahren noch minderjährigen Zeugin an einem auswärtigem Ortstermin betraf, war vom Ausgangsgericht den Eltern, die ihre Zustimmung nicht erteilt hatten, das Aufenthaltsbestimmungsrecht für die Dauer der Reise entzogen und auf einen Pfleger übertragen worden. Das $O L G$ Düsseldorf rügte diese Maßnahme als nicht notwendig, da zumindest bei ausreichender geistiger Reife das Erscheinen des aussagebereiten Minderjährigen auch ohne Beschränkung des Aufenthaltsbestimmungsrechtes erzwungen werden könne ${ }^{6}$. Eine Begründung enthält die Entscheidung nicht, obgleich ihr im Ergebnis zuzustimmen ist.

Die Pflicht zum Erscheinen wird gemäß § 386 III ZPO nur durch die Ausübung eines Zeugnisverweigerungsrechtes zumindest vorläufig suspendiert $^{7}$. Bei der Entscheidung über die Ausübung des Weigerungsrechtes sind die gesetzlichen Vertreter jedoch nur in Ausnahmefällen beteiligt. Demgegenüber würde ein Rückgriff auf das Aufenthaltsbestimmungsrecht, das seiner Intention nach nicht den kurzfristigen Aufenthaltsort, sondern Wohnsitz und -ort meint ${ }^{8}$, zu einer dem Sinn und Zweck des Zeugnisverweigerungs-

\footnotetext{
${ }^{5}$ Skupin, MDR 1965, 865 ff hält das Aufenthaltsbestimmungsrecht auch im Zusammenhang mit Untersuchungen nach $\S 81 \mathrm{c}$ StPO für bedeutsam.

${ }^{6}$ OLG Düsseldorf, FamRZ 1973, $547 \mathrm{f}$.

${ }^{7}$ Bruns, Rn 184 a; Niese, S. 143.

${ }^{8}$ MünchKomm-Hinz, §1631 Rn 18; Soergel-Strätz, §1631 Rn 17; Staudinger-Donau, $\S 1631 \mathrm{Rn} 315$. Soweit ersichtlich wurde der Konflikt zwischen Erscheinenspflicht und Aufenthaltsbestimmungsrecht der Eltern mit Ausnahme des genannten Urteils des OLG Düsseldorf bisher nicht problematisiert.
} 
rechtes und der gesetzlichen Zeugenpflichten des Minderjährigen widersprechenden Beteiligung der Eltern in jedem Fall führen.

Das Aufenthaltsbestimmungsrecht leitet sich aus dem Recht der elterlichen Sorge gemäß § 1626 BGB ab. Das Sorgerecht der Eltern besteht jedoch nicht unbeschränkt ${ }^{9}$, zumal es auch nicht in vollem Umfang, sondern lediglich in seinem Kernbereich an der Grundrechtsgarantie des Art. 6 II GG teilnimmt, der seinerseits auch verfassungsimmanenten Schranken unterliegt ${ }^{10}$. Die Schranken ergeben sich dabei nicht nur aus der Zielsetzung der elterlichen Sorge selbst, sondern auch aus dem Verhältnis zu anderen, gleichrangigen Rechtsgütern. So enthält beispielsweise die - landesrechtlich normierte - Schulpflicht ${ }^{11}$ eine öffentlich-rechtliche Pflicht, hinter der das elterliche Bestimmungsrecht zurücktritt ${ }^{12}$.

Die Zeugenpflicht ist ebenfalls eine öffentlich-rechtliche Pflicht, die dem hochrangigen öffentlichen Interesse an der Rechtspflege dient. Dies rechtfertigt eine Beschränkung des Aufenthaltsbestimmungsrechtes. Die Eltern sind nicht nur zur Duldung verpflichtet, sondern sie haben das ordnungsgemäß geladene Kind ${ }^{13}$ zum tatsächlichen Erscheinen anzuhalten und gegebenenfalls zum Termin mitzubringen ${ }^{14}$.

\footnotetext{
${ }^{9}$ Beitzke, $\S 27$ IV 1 nimmt es als geradezu selbstverständlich an, daß das elterliche Recht der Aufenthaltsbestimmung gegenüber öffentlich-rechtlichen Aufenthaltspflichten zurücktritt.

${ }^{10}$ BVerfGE 24, 119, 143 zu den Schranken des Art. 6 II GG; Maunz in Maunz-Dürig, Art. 6 $\mathrm{Rn} 24$ b und $\mathrm{Rn} 26$.

${ }^{11}$ Z.B. Art. 35 IV 2 BayEUG: "Er (der Schüler) hat insbesondere die Pflicht, die Schule zu besuchen und sich am Unterricht zu beteiligen." und Art. 54 BayEUG: "Die Erziehungsberechtigten sind verpflichtet, um die gewissenhafte Erfüllung der schulischen Pflichten ... besorgt zu sein ...".

${ }^{12}$ Maunz in Maunz-Dürig, Art. 7 Rn 21 g; Erman-Michalski, § 1631 Rn 5.

${ }^{13}$ Zur Ladung oben $\S 4$.

${ }^{14}$ OLG Hamm, NJW 1965, 1613; OLG Saarbrïcken, MDR 1974, 859; Skupin, MDR 1965, 865, 876. Zur Verhängung von Ordnungsmitteln gegen die Eltern, die das Kind am Erscheinen hindern s.o. \& 4 C III.
} 


\section{Die Aussagepflicht}

\section{Inhalt und Umfang der Aussagepflicht}

Die eigentliche Zeugenpflicht ist unzweifelhaft die Pflicht zur wahrheitsgemäßen und vollständigen Aussage; sie umfaßt die Beantwortung aller Fragen zu dem in der Ladung gem. §377 II Ziff. 2 ZPO benannten Beweisthema sowie gem. § 395 II ZPO Fragen zu den persönlichen Verhältnissen des Zeugen ${ }^{15}$. Soweit es bei der Ausübung eines Zeugenrechts auf eine bestimmte geistige Reife ankommt, wird das erkennende Gericht ${ }^{16}$, das die Beweisaufnahme durchführt, nicht umhin kommen, gegebenenfalls durch geeignete Fragen festzustellen, ob bei einem Minderjährigen diese Voraussetzung vorliegt. Das Gericht ist hierbei aber gerade in den Fällen, in denen Zweifel an der Verstandesreife des Zeugen bestehen, durch den Verhältnismäßigkeitsgrundsatz und die Pflicht zur Wahrung der Menschenwürde ${ }^{17}$ zur Zurückhaltung bei der Prüfung der geistigen Fähigkeiten verpflichtet.

\section{Befreiung von der Aussagepflicht bei fehlender Verstandesreife}

a) Die Ansicht von Karl Peters

Die Aussagepflicht wird durch die Ausübung eines Zeugnisverweigerungsrechtes zunächst vorläufig, bei Verzicht der Parteien auf den Zeugen oder nach rechtskräftiger Entscheidung über die Rechtmäßigkeit der Weigerung endgültig aufgehoben ${ }^{18}$. Zum Strafprozeßrecht hat Karl Peters die Ansicht entwickelt, die Vernehmung eines Zeugen sei gänzlich ausgeschlossen, wenn dem Zeugen die für die Lösung der Konfliktslage durch wirksamen Verzicht auf sein Zeugnisverweigerungsrecht erforderliche Verstandes-

\footnotetext{
${ }^{15}$ Bruns, Rn 184 b, c; Rosenberg-Schwab, § $123 \mathrm{~V}$.

${ }^{16}$ BGHSt 14, 159, 160; BayObLG NJW 1967, 206, 209.

${ }^{17}$ Vgl. allgemein Stein-Jonas-Schumann, Vor § $373 \mathrm{Rn} 35$; derselbe, §395 Rn 3. Zur Durchführung der Vernehmung unter Berücksichtigung des Zeugen- und Minderjährigenschutzes vgl. unten $\S 8 \mathrm{~B}, \mathrm{C}$.

${ }^{18}$ Zur Ausübung des Zeugnisverweigerungsrechtes vgl. § 6.
} 
reife fehle ${ }^{19}$. Danach käme es bei einem nicht einsichtsfähigen minderjährigen Zeugen ${ }^{20}$ nicht auf die wirksame Ausübung eines Weigerungsrechtes an, weil bereits bei Vorliegen der Weigerungsvoraussetzungen eine dauerhafte Befreiung von der Aussagepflicht gegeben wäre.

Peters begründet seine Meinung damit, daß eine Zustimmung der gesetzlichen Vertreter nicht möglich sei, weil ihre Interessen an einer Aussage denen des Kindes völlig entgegengesetzt sein können und die als Folge der Aussage drohenden seelischen Belastungen des Zeugen nicht von Dritten auferlegt werden dürfen. Die gerichtliche Fürsorgepflicht gebiete daher den Verzicht auf das Beweismittel ${ }^{21}$. Dafür spricht nach Peters auch die Vermeidung praktischer Schwierigkeiten bei der Bestimmung der zur Zustimmungserteilung befugten gesetzlichen Vertreter sowie der Ausschluß der Gefahr einer Beeinflussung der Aussage ${ }^{22}$.

\section{b) Stellungnahme}

Der dargestellten Ansicht kann nicht beigetreten werden. Der Strafgesetzgeber hat durch die Einführung des § 52 II StPO nunmehr entschieden, daß eine Zeugenvernehmung auch bei Verstandesunreife nicht ipso iure ausgeschlossen ist. Die Bedenken, die sich aus der Beeinflussungsmöglichkeit des Zeugen ergeben, können das Beweisinteresse nicht aufwiegen. Dies gilt ebenso im Zivilprozeß.

Darüberhinaus ist die Argumentation von Peters insoweit bedenklich, als er die Fürsorgepflicht in der Form des Verzichts auf das Beweismittel nur weigerungsberechtigten Zeugen zugute kommen lassen will. Das Fürsorgebedürfnis beruht in erster Linie auf der Unreife des Minderjährigen. Zwar wirkt sich die Entscheidungsunreife nur dann aus, wenn eine Entscheidung zu treffen ist. Dies ist wiederum nur bei gegebenem Zeugnisverweigerungsrecht der Fall. Angesichts der Tatsache, daß gegen schuldunfähige Minder-

\footnotetext{
${ }^{19}$ K. Peters, Gutachten, S. 115 ff; derselbe, JR 1970, 308 f. Ähnlich will Moritz, S. 412 auf die Ladung und damit auch auf die Vernehmung solcher Zeugen verzichten. Hauser, S. $68 \mathrm{f}$ hält in Zivilverfahren einen Verzicht auf Kinder als Beweismittel für denkbar.

${ }^{20}$ Zur Einsichtsfähigkeit als Ausübungsvoraussetzung unten $\S 6$ A.

${ }^{21}$ K. Peters, JR 1970, 308 f.

${ }^{22}$ K. Peters, Gutachten, S. 116 f.
} 
jährige Ordnungs- und Zwangsmittel nicht verhängt werden dürfen ${ }^{23}$, besteht rein faktisch ein Entscheidungsspielraum bei jedem dieser Zeugen. Darüberhinaus läßt der Verzicht auf das Beweismittel keine Möglichkeit zur Prüfung offen, ob der Schutz des Minderjährigen nicht auf andere Weise gewahrt werden kann, ohne die Parteien übermäßig in ihrem rechtlichen Gehör und Beweismöglichkeiten einzuschränken ${ }^{24}$.

Zum anderen geht auch die Annahme fehl, daß die seelischen Belastungen dem Zeugen nicht von Dritter Seite auferlegt werden dürfen. Diese Gefahr besteht nicht, weil die Zustimmung der Eltern weder Aussagepflicht noch Aussagezwang des Kindes ${ }^{25}$ begründet. Die Einverständniserklärung der Eltern hat daher nur bei fortdauernder Aussagebereitschaft des Kindes Auswirkungen.

Es ist daher festzuhalten, daß die fehlende Verstandesreife die Aussagepflicht nicht berührt. Eine Befreiung tritt erst mit wirksamer Zeugnisverweigerung ein. Dies gilt im übrigen auch dann, wenn das Gericht wegen fehlender Zeugeneignung bereits den Beweisantrag auf Vernehmung des Zeugen abgelehnt hat ${ }^{26}$. Mangels Ladung des Zeugen konkretisieren sich die Zeugenpflichten gar nicht, so daß es keiner Befreiung von der Aussagepflicht bedarf.

\section{Die Eidespflicht}

Unter den Voraussetzungen des $\S 391 \mathrm{ZPO}$ ist jeder Zeuge auch verpflichtet, seine Aussage zu beeiden oder zumindest nach $\S 484$ ZPO eidesgleich zu bekräftigen ${ }^{27}$.

Anders als die Zeugnisfähigkeit und die Zeugeneignung ist die Eidesfähigkeit ausdrücklich gesetzlich geregelt. Nach § 393 ZPO dürfen Personen,

\footnotetext{
${ }^{23}$ Vgl. unten $\S 5 \mathrm{C}$.

${ }^{24} \mathrm{Zu}$ denken ist hier insbesondere an die nichtöffentliche Vernehmung unter Ausschluß auch der Parteien; vgl. unten $\S 8 \mathrm{~B}, \mathrm{C}$.

${ }^{25}$ BGHSt 14, 159, 160; 21, 303, 306; OLG Stuttgart, FamRZ 1965, 515, 516; Staudinger-Donau, $\S 1626 \mathrm{Rn} 75$; Stein-Jonas-Schumann, § $383 \mathrm{Rn} 7$; ferner unten § 5 C I.

${ }^{26} \mathrm{Im}$ Einzelnen dazu oben $\S 3 \mathrm{~B}$.

${ }^{27}$ Zöller-Stephan, § $391 \mathrm{Rn} 1$.
} 
die nicht die Verstandesreife besitzen, die Bedeutung des Eides zu verstehen sowie generell alle Minderjährigen vor Vollendung des 16. Lebensjahres nicht vereidigt werden ${ }^{28}$.

Sofern diese Ausnahmen nicht gegeben sind, liegt die Vereidigung im Ermessen des Gerichts ${ }^{29}$. Erst mit der Anordnung entsteht die Eidespflicht im konkreten Fall. Die Beeidigung erfolgt stets nach Erstattung der Aussage, da die Zivilprozeßordnung nur noch den Nacheid kennt ${ }^{30}$.

\section{B. Die Weigerungsrechte als Einreden gegen Zeugnispflichten}

\section{Rechtliche Einordnung}

\section{Die Zeugnisverweigerungsrechte}

Das Zeugnisverweigerungsrecht läßt sich als eine Art prozessuale Einrede qualifizieren ${ }^{31}$, deren Geltendmachung die Möglichkeit einer Befreiung von Erscheinens-, Aussage- und Eidespflicht schafft. Die Befreiung tritt daher nicht bereits bei Vorliegen der objektiven Voraussetzungen der $\S 383 \mathrm{ff}$ ZPO ein, sondern erst, wenn der Zeuge eine entsprechende Erklärung, die den Erfordernissen des $\S 386$ ZPO zu entsprechen hat, abgibt.

Orlowsky vertritt die Ansicht, daß die Erklärung über die Ausübung eines Zeugnisverweigerungsrechtes wegen des öffentlich-rechtlichen Charakters

\footnotetext{
${ }^{28}$ Eine inhaltlich übereinstimmende Regelung enthält $\S 60 \mathrm{Nr}$. 1 StPO für das Strafverfahren. Infolge der Einschränkung der Eidesfähigkeit entfallen größtenteils die Probleme, die sich im Zusammenhang mit der selbständigen Ausübung des Zeugnisverweigerungsrecht und der Belehrung des Minderjährigen ergeben. Dazu auch unten § 5 B I 2.

${ }^{29}$ Baumbach-Lauterbach-Hartmann, § 391 Anm. 2 D; Stein-Jonas-Schumann, § 391 Rn 1, 6 f; Thomas-Putzo, § 391 Anm. 1.

${ }^{30}$ Zum Nacheid auch Rosenberg-Schwab, § 123 VI; Stein-Jonas-Schumann, § 392 Rn 1; ferner unten $\S 5$ B I 2.

${ }^{31}$ Niese, S. 143 f; Rosenberg-Schwab, § 123 V 2. Goldschmidt, ZPR, $\$ 472 \mathrm{~b}$ bezeichnet das Zeugnisverweigerungsrecht als Beweishindernis; ähnlich auch E. Peters, ZZP 77, 444, 459, der von einer "Rechtswohltat für den Zeugen" spricht.
} 
der Zeugenpflichten eine ebenfalls öffentlich-rechtliche Willenserklärung $\operatorname{sei}^{32}$. Damit sind jedoch keine weiteren Schlußfolgerungen verbunden, die eine Beantwortung der offenen Fragen gestatten, die sich im Zusammenhang mit einem minderjährigen Zeugen stellen. Offen bleibt nach dieser Ansicht auch, nach welchem Recht die Ausübungserklärung in anderen Rechtswegen, etwa im Zivilprozeß oder im Verwaltungsverfahren zu beurteilen wäre.

Die Qualifizierung als öffentlich-rechtliche Willenserklärung erschwert den Blick darauf, daß es sich um ein rein prozessuales Problem handelt ${ }^{33}$, wann und unter welchen Voraussetzungen ein Minderjähriger ein Zeugnisverweigerungsrecht wirksam selbständig aussüben kann.

Das Zeugnisverweigerungsrecht hat auch keinen Doppelcharakter in dem Sinne, daß es sich um eine materiell-rechtliche und prozessuale Handlung zugleich handelt ${ }^{34}$. Soweit damit die Vorstellung verbunden ist, daß neben den prozessualen Voraussetzungen weitere Ausübungsvoraussetzungen vorliegen müssen, liegt darin eine gesetzlich nicht geregelte und sachlich nicht gerechtfertigte Einschränkung des Zeugnisverweigerungsrechts ${ }^{35}$.

Problematischer ist der Hinweis, daß die Geltendmachung eines Zeugnisverweigerungsrechts im Prozeß eine Prozeßhandlung darstelle ${ }^{36}$. Danach müßten die Prozeßhandlungsvoraussetzungen vorliegen, um die Einrede gegen die Zeugenpflichten wirksam erheben zu können. Dazu gehören unter anderem die Partei- und die Prozeßfähigkeit ${ }^{37}$. Bei konsequenter Betrach-

\footnotetext{
${ }^{32}$ Orlowsky, S. 47 zur StPO.

${ }^{33}$ Dies gilt auch für die Feststellung, daß das Prozeßrecht als solches öffentliches Recht ist, da sich daraus ebenfalls keine konkreten Folgen ableiten und begründen lassen; ähnlich SteinJonas-Schumann, Einl. Rn 95.
}

${ }^{34}$ So aber Niese, S. 53 u. 142 f und Orlowsky, S. 47 ff, die dies durch die Bezeichnung als "doppelfunktionelle Prozeßhandlung" zum Ausdruck bringen.

${ }^{35}$ Allgemein zum Doppelcharakter von Prozeßhandlung und materiell-rechtlicher Handlung Stein-Jonas-Leipold, Vor $\$ 128$ Rn $254 \mathrm{ff}$, der eine Kumulation verschiedener Voraussetzungen ablehnt; ebenso Rosenberg-Schwab, § 63 I a.E.

${ }^{36}$ So Bosch, S. 39; Gebhardt, S. 79; E. Schmidt, JR 59, 369 f (zur StPO). Zum Begriff der Prozeßhandlung allgemein Rosenberg-Schwab, §63 IV; Stein-Jonas-Leipold, Vor § 128 Rn 157 ff m.w.N.; OLG Stuttgart, NJW 1971, 2237, 2239.

${ }^{37}$ Bosch, S. 40; Grundmann, S. 11; Stein-Jonas-Leipold, Vor $\S 128$ Rn 183; Thomas-Putzo, Einl. III 2; Zöller-Vollkommer, Vor § $50 \mathrm{Rn} 17 \mathrm{ff}$. 
tung könnte ein Minderjähriger nie ein Zeugnisverweigerungsrecht selbständig geltend machen, weil ihm regelmäßig die Prozeßfähigkeit fehlt ${ }^{38}$.

Diese Konsequenz wird jedoch weder von der Literatur noch von der Rechtsprechung gezogen ${ }^{39}$. Die Prozeßfähigkeit als Ausübungsvoraussetzung würde dem Wesen des Zeugnisverweigerungsrechtes als höchstpersönliche Entscheidung nicht gerecht, weil die Folgen der Entscheidung über Aussage oder Verweigerung und der damit verbundene Gewissenskonflikt von dem Erreichen der Prozeßfähigkeit unabhängig sind ${ }^{40}$. Im übrigen definiert § 51 ZPO Prozeßfähigkeit als Fähigkeit einer Partei, vor Gericht zu stehen. Dies spricht ebenfalls gegen eine Erstreckung dieser Voraussetzungen auf Dritte, soweit sie keine parteiähnliche Stellung inne haben ${ }^{41}$.

Nach Meinung von Bosch wird der Zeuge durch die Ausübung eines Zeugnisverweigerungsrechts aber Verfahrensbeteiligter im weiteren Sinne ${ }^{42}$. Der Minderjährige sei insoweit teilprozeßfähig. Die von Bosch zum Vergleich herangezogenen Vorschriften betreffen jedoch Situationen, in denen der Minderjährige tatsächlich Beteiligter ist. Dies gilt etwa für die minderjährige Ehefrau im Eherechtsstreit oder für Streitigkeiten des Minderjährigen im Zusammenhang mit einem Lebensbereich im Sinne der $\S \S 112,113 \mathrm{BGB}^{43}$. Die bloße Ausübung eines Zeugnisverweigerungsrechts macht den Zeugen demgegenüber noch nicht zum Verfahrensbeteiligten, sie soll seine Mitwirkung am Verfahren, d.h. hier an der Beweisaufnahme gerade verhindern. Etwas anderes kommt allenfalls dann in Betracht, wenn über die Weigerung des Zeugen ein Zwischenstreit geführt wird. In diesem Verfahren ist der Zeuge Beteiligter ${ }^{44}$.

\footnotetext{
${ }^{38}$ Zur Prozeßfähigkeit und zum Begriff des Minderjährigen s. oben $\S 1$.

${ }^{39}$ Auch das Gesetz stellt in $\S 52$ II StPO auf die Einsichtsfähigkeit, nicht aber auf sonstige prozessuale Voraussetzungen ab. Zur Erklärung des Zeugen im Strafverfahren, aussagen zu wollen, wie hier K. Peters, JR 1970, 308.

${ }^{40} \mathrm{Zum}$ Charakter des Zeugnisverweigerungsrechtes als höchstpersönliches Recht OLG Düsseldorf, FamRZ 1973, 547; Orlowsky, S. 59 ff; Soergel-Strätz, §1629 Rn 18; SteinJonas-Schumann, § $383 \mathrm{Rn} 2$.

${ }^{41}$ Zöller-Stephan, Vor § $128 \mathrm{Rn} 17$.

${ }^{42}$ Bosch, S. 39. Zum Versuch Bosch's, für den Beginn der Zeugenmündigkeit eine feste Altersgrenze zu begründen, vgl. unten § 5 B II 1.

${ }^{43}$ Gegen die Annahme einer prozessualen Teilmündigkeit auch Moritz, S. $411 \mathrm{f}$.

${ }^{44}$ Stein-Jonas-Leipold, Vor $\S 128 \mathrm{Rn} 167$. Zum Zwischenstreit über das Zeugnisverweigerungsrecht unten $\S 10 \mathrm{~A}$.
} 
Auf die Beurteilung der Ausübung eines Zeugnisverweigerungsrechtes als Prozeßhandlung sollte m.E. verzichtet werden. Sie ist ähnlich der Qualifizierung als öffentlich-rechtliche Willenserklärung hier ohne tatsächlichen Erkenntniswert und kann zu dem Mißverständnis führen, die Prozeßhandlungsfähigkeit als notwendige Voraussetzung der Ausübung des Zeugnisverweigerungsrechtes anzusehen. Die Prozeßhandlungsfähigkeit ist aber nur bei Prozeßhandlungen im engeren Sinne, also solcher der Parteien erforderlich. Der Begriff der Prozeßhandlung sollte daher von vorneherein auf Handlungen der Parteien und des Gerichts beschränkt bleiben und allenfalls auf Personen mit parteiähnlicher Stellung, etwa dem Zeugen im Zwischenstreit über sein Zeugnisverweigerungsrecht nach §§ 387, 388 ZPO erstreckt werden $^{45}$. Auf Prozeßhandlungsfähigkeit kommt es daher bei der Erklärung über die Zeugnisverweigerung nicht an.

\section{Das Eidesverweigerungsrecht}

Es ist anerkannt, daß die Eidesleistung unter denselben Voraussetzungen verweigert werden kann, die auch zur Zeugnisverweigerung berechtigen ${ }^{46}$. Damit ist grundsätzlich die selbständige Eidesverweigerung gemeint, weil bei Verweigerung bereits der Aussage eine Beeidigung gar nicht vorgenommen werden könnte ${ }^{47}$. Die selbständige Eidesverweigerung ist gesetzlich nicht geregelt ${ }^{48}$, doch ergibt sich ihre Zulässigkeit aus § 390 I ZPO und aus $\S 63 \mathrm{StPO}^{49}$.

\footnotetext{
${ }^{45}$ Dies entspricht auch im wesentlichen dem Gebrauch des Begriffs im Schriftum, Baumbach-Lauterbach-Hartmann, Grundz. § 128 Anm. 5 B; Zöller-Stephan, Vor § 128 Rn 17. Noch enger Thomas-Putzo, Einl. III 1, wonach Prozeßhandlungen nur Handlungen der Parteien, der Nebenintervenienten und deren Vertreter sind. Handlungen des Gerichtes wären damit ebenfalls keine Prozeßhandlungen.

${ }^{46}$ BGHZ 43, 368; Stein-Jonas-Schumann, § 392 Rn 6; Zöller-Stephan, § 391 Rn 1.

${ }^{47}$ Anders nur beim Voreid, der aber bereits im Jahre 1909 aus der ZPO gestrichen wurde; dazu Bruns, Rn 186 a.

${ }^{48}$ Bis 1933 galt in $\S 393$ Satz 1 Nr. 3 und 4 ZPO die Regelung, daß Zeugen, denen nach $\S 383$ I Nr. 1 bis 3 , $\$ 384 \mathrm{Nr} .1$ und $2 \mathrm{ZPO}$ ein Zeugnisverweigerungsrecht zustand, von dem sie jedoch keinen Gebrauch gemacht haben, den Eid verweigern konnten. Darüberhinaus hatten diejenigen, die am Ausgang des Verfahrens ein rechtliches Interesse hatten, ebenfalls ein Eidesverweigerungsrecht. Allerdings konnte das Gericht nach § 393 Satz 2 ZPO trotz der Weigerung die Beeidigung anordnen. Diese Regelung wurde durch das "Gesetz zur Änderung des
} 
Die Eidesverweigerung kommt nur bei gegebener Eidespflicht in Betracht, mithin nur bei Minderjährigen nach Vollendung des 16. Lebensjahres und hinreichender Verstandesreife im Sinne des $\$ 393$ ZPO. Eine besondere Darstellung des Eidesverweigerungsrechts neben dem Zeugnisverweigerungsrecht erübrigt sich daher, weil bei Vorliegen der Eidespflicht gleichzeitig die Voraussetzungen für eine Gleichstellung des Minderjährigen mit einem Volljährigen vorliegen ${ }^{50}$. Auch hinsichtlich der rechtlichen Einordnung kann mangels grundlegender Unterschiede auf die entsprechenden Ausführungen beim Zeugnisverweigerungsrecht verwiesen werden ${ }^{51}$.

\section{Gesetzliche Regelung in der Zivilprozeßordnung}

\section{Die Zeugenmündigkeit im geltenden Recht nach der Auffassung von F. W. Bosch}

Das Recht zur Verweigerung einer Zeugenaussage ist in den $\S \S 383 \mathrm{ff}$ ZPO abschließend ${ }^{52}$ geregelt. Eine besondere Regelung über die Ausübung dieses Rechts durch Minderjährige enthalten diese Vorschriften nicht. Diese Gesetzeslücke ${ }^{53}$ betrifft neben dem Zeugnisverweigerungsrecht selbst auch die nach § 383 II ZPO zwingend vorgeschriebene Belehrung des Zeugen.

Einen Versuch zur Schließung der Regelungslücke hat F.W. Bosch in seiner 1963 erschienenen Monographie "Grundsatzfragen des Beweisrechts"

Verfahrens in bürgerlichen Rechtsstreitigkeiten" vom 27.10.1933 zugunsten der heute geltenden Fassung geändert. Die Änderung erfolgte, weil die Beeidigung überhaupt in das Ermessen des Gerichts gestellt wurde, während zuvor ein Zeuge nur ausnahmsweise unbeeidigt bleiben durfte. Eine Abschaffung des Eidesverweigerungsrechts war durch die Gesetzesänderung somit nicht beabsichtigt; dazu Rosenberg, ZZP 58, 283, $311 \mathrm{ff}$.

${ }^{49}$ Baumbach-Lauterbach-Hartmann, § 391 Anm. 2 C; Stein-Jonas-Schumann, § 392 Rn 6.

${ }^{50}$ Zur Gleichstellung s.u. § 6 B.

${ }^{51}$ Oben $\S 5$ B I 1.

${ }^{52}$ Baumbach-Lauterbach-Hartmann, Einf. Vor §§ 383-389 Anm. 1; Stein-Jonas-Schumann, $\S 383 \mathrm{Rn}$ 13, 16; Thomas-Putzo, § 383 Anm. 1; Wieczorek, § 383 Anm. A.

${ }^{53}$ Bosch, S. 36; ebenso Orlowsky, S. 10 zur StPO vor 1974. 
unternommen. Seiner Ansicht nach kann jeder Zeuge nach Vollendung des 14. Lebensjahres die Rechte und Pflichten eines Zeugen selbständig wahrnehmen. Mit diesem Alter setze die Zeugenmündigkeit ein, der Minderjährige sei dann insoweit auch prozeß- und prozeßhandlungsfähig ${ }^{54}$. Die genannte Altersgrenze, die er als "Zeugendiskretionsalter" bezeichnet, hält Bosch bereits de lege lata für verbindlich ${ }^{55}$. Er begründet dies mit einer von ihm angenommenen besonderen Bedeutung des Erreichens dieses Lebensalters, die er durch eine Analogie zu Vorschriften aus dem Gesetz zur religiösen Kindererziehung ( $\$ 5$ RKEG - Religionsmündigkeit), dem Bürgerlichem Recht ( $\$ 1751$ II BGB a.F., jetzt § 1746 BGB - Zustimmung zum Adoptionsvertrag), dem Gesetz über die Angelegenheiten der freiwilligen Gerichtsbarkeit (§59 FGG - Beschwerdebefugnis) und nicht zuletzt dem Strafrecht (§£ 1, 3 JGG - Strafmündigkeit) nachzuweisen versucht ${ }^{56}$.

\section{Stellungnahme}

Die Thesen Boschs haben viel Beachtung, aber kaum Zustimmung gefunden, soweit es um die Verbindlichkeit der Altersgrenze für die Zeugenmündigkeit bereits im geltenden Recht geht ${ }^{57}$. Zum einen, so die Gegenargumentation, handele es sich bei der genannten Altersgrenze um eine willkürlich gezogene, für die es im Gesetz keinen Anhaltspunkt gebe ${ }^{58}$. Zum anderen obliege es allein dem Gesetzgeber, eine für das Prozeßrecht nicht unwichtige Altersgrenze festzulegen ${ }^{59}$. Das gilt umso mehr, als der Gesetzgeber für das Strafverfahrensrecht nun eine entsprechende gesetzliche Regelung geschaffen hat, dabei aber gerade keine feste Altersgrenze festgelegt wurde ${ }^{60}$. Den gleichen Bedenken muß auch die Ansicht Donaus begegnen, der unter Berufung auf die Eidesmündigkeit nach § 393 ZPO und die

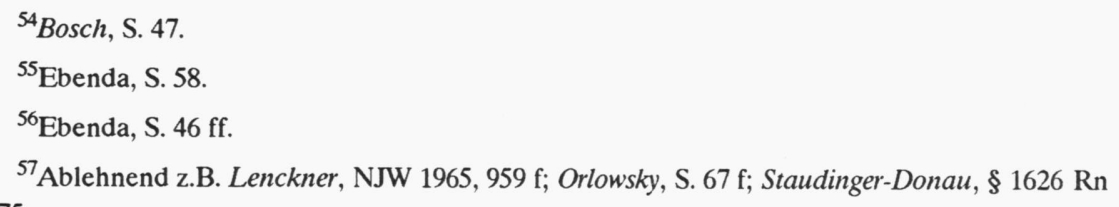
75.

${ }^{58}$ Bruns, ZZP 77, 318; Rosenberg-Schwab, § 123 V 3 Fn 24; Staudinger-Donau, § 1626 Rn 55 Fn 2.

${ }^{59}$ OLG Stuttgart, FamRZ 1965, 515, 516; Grundmann, S. 92; Hampel, FamRZ 1964, 124, 128; MünchKomm-Hinz, § 1626 Rn 46.

${ }^{60} \mathrm{Zu} \S 52$ II StPO unten $\S 5$ B III. 
Zulässigkeit der Vernehmung der minderjährigen Partei gemäß § 455 II ZPO für eine Altersgrenze von 16 Jahren plädiert, ab der die Rechte eines Zeugen selbständig ausgeübt werden dürfen ${ }^{61}$.

Die besondere und vom Gesetzgeber in anderen Bereichen durchaus anerkannte Schutzbedürftigkeit des Minderjährigen ${ }^{62}$ läßt es, trotz der Ablehnung der eben dargestellten Meinungen, erforderlich erscheinen, auch den Minderjährigen als Zeugen stärker zu schützen. Nur so kann der Gewissenskonflikt, vor dem jedes Zeugnisverweigerungsrecht schützen will, in einer die verschiedenen Interessen berücksichtigenden Weise zum Ausgleich gebracht werden.

\section{Gesetzliche Regelung in der Strafprozeßordnung, insbesondere § 52 II StPO}

Auf die Zeugnisverweigerungsrechte, die in der Strafprozeßordnung geregelt sind, soll hier nur im Zusammenhang mit § 52 II StPO eingegangen werden. Diese Vorschrift wurde im Jahre 1974 durch das 9. Strafrechtsänderungsgesetz neu geschaffen ${ }^{63}$. Es stellt sich die Frage, ob eine analoge Anwendung auf den Zivilprozeß möglich und zweckmäßig ist. Darüberhinaus ist aber die Entwicklung der Rechtsprechung kurz darzustellen, da die hier deutlich gewordenen Gegensätze und Konflikte auch im Zivilverfahren von Bedeutung sind.

Nach $\S 52$ II StPO darf ein aussagebereiter Minderjähriger, dem die zum Verständnis der Bedeutung des Zeugnisverweigerungsrechtes erforderliche Verstandesreife fehlt, nur dann als Zeuge vernommen werden, wenn auch die gesetzlichen Vertreter der Vernehmung zustimmen. Die Zustimmungs-

\footnotetext{
${ }^{61}$ So Staudinger-Donau, § $1626 \mathrm{Rn} 75$; dazu auch unten B II 2.

${ }^{62}$ Der Minderjährigenschutz im Prozeßrecht ist im Vergleich zum materiellen Recht lückenhaft, Dehmer, S. $11 \mathrm{ff}, 14 \mathrm{ff}$. Moritz, S. $42 \mathrm{ff}$ weist auf den anthropologischen Ursprung des Gedankens der besonderen Schutzbedürftigkeit hin. Die Schutzbedürftigkeit rechtfertigt nach BVerfGE 24, 119, 144 die Anerkennung der Verantwortung der Eltern und der damit verbundenen Rechte. Erst die Schutz- und Hilfsbedürftigkeit macht das Institut der elterlichen Sorge, aber auch das "Wächteramt des Staates" erforderlich.
}

${ }^{63}$ BGBI. I, S. 3393 vom 9.12.1974. 
befugnis der gesetzlichen Vertreter entfällt, wenn auch nur einer von ihnen selbst Beschuldigter des Strafverfahrens ist. In diesem Fall ist gemäß $\S 1909$ BGB ein Ergänzungspfleger zu bestellen, dessen Entscheidung die der Eltern ersetzt ${ }^{64}$. Hat der Minderjährige dagegen die erforderliche Verstandesreife, ist er nach überwiegender Meinung, die sich auf einen Umkehrschluß aus $\S 52$ II 1 StPO stützt, zur selbständigen Ausübung des Zeugnisverweigerungsrechts befugt ${ }^{65}$.

Die besondere Schutzbedürftigkeit von Minderjährigen, auch im Zusammenhang mit der Ausübung eines Zeugnisverweigerungsrechts, war keineswegs von Anfang an selbstverständlich. So hielt das Reichsgericht in seiner ersten Entscheidung zu diesem Problem ${ }^{66}$ die Belehrung des jugendlichen Zeugen für erforderlich und ausreichend. Auf das Verständnis des Minderjährigen bezüglich seines Zeugnisverweigerungsrechts sollte es nicht ankommen, solange nur der Pflicht ordnungsgemäßer Belehrung genügt war. Diese Rechtsprechung hielt das Reichsgericht in einer weiteren Entscheidung aufrecht, in der es den Einwand der Revision zurückwies, ein beschränkt Handlungs- und Zeugnisfähiger könne eine Erklärung über die Nichtausübung des Zeugnisverweigerungsrechts nicht rechtsverbindlich abgeben. Zur Begründung wurde angeführt, es komme lediglich auf die gesetzlich geforderte Belehrung an; ein ausdrücklicher Verzicht auf das Zeugnisverweigerungsrecht sei im übrigen nicht erforderlich ${ }^{67}$.

Auch der Bundesgerichtshof lag zunächst auf dieser Linie, nachdem er wegen der Höchstpersönlichkeit des Zeugnisverweigerungsrechts und der Qualifizierung der Ausübung als Prozeßhandlung eine Vertretung des Minderjährigen für ausgeschlossen hielt ${ }^{68}$. Erst in einer weiteren Entscheidung im Jahre 1958 änderte sich die Rechtsprechung. Nach Auffassung des Bundesgerichtshofs soll § 52 I StPO dem Zeugen die Möglichkeit geben, den Konflikt, einen Familienangehörigen durch eine wahrheitsgemäße Aussage zu belasten oder aber die Unwahrheit sagen zu müssen, durch Verweigerung der Aussage zu lösen; nur auf diese objektive Konfliktslage komme es

\footnotetext{
${ }^{64}$ Kleinknecht/Meyer, § $52 \mathrm{Rn} 20$; KMR-Paulus, § $52 \mathrm{Rn} 24$; Löwe-Rosenberg-Dahs, § $52 \mathrm{Rn}$ 32.

${ }^{65}$ Löwe-Rosenberg-Dahs, § $52 \mathrm{Rn} 22$ m.w.N.; zur Gleichstellung des Minderjährigen mit ausreichender Verstandesreife mit einem Volljährigen auch unten $\S 6 \mathrm{~B}$.

${ }^{66}$ RGSt 4, 398 v. 14.7.1881.

${ }^{67}$ RGSt $12,403,404$ v. 17.9.1885.

${ }^{68}$ BGH 5 StR 394/54 v. 30.11.1954 zit. bei BayObLG NJW 67, 206, 207.
} 
$a^{69}$. Hat der Zeuge nicht die Verstandesreife, die Konfliktslage zu begreifen, so müsse er bei der Entscheidung durch einen vom Vormundsschaftsgericht zu bestellenden Ergänzungspfleger vertreten werden. Nur dieser Vertreter sei dann über das Zeugnisverweigerungsrecht zu belehren, da sich die Vertretung auch darauf erstrecke ${ }^{70}$. In der Folgezeit wurde die Judikatur des Bundesgerichtshofs differenzierter. Als wesentliche Ergänzung war die Feststellung anzusehen, ein Minderjähriger ohne ausreichende Verstandesreife dürfe auch trotz Zustimmung der gesetzlichen Vertreter das Zeugnis verweigern ${ }^{71}$. Damit wurde anerkannt, daß die Zustimmung der gesetzlichen Vertreter keine Aussagepflicht zu Lasten des Minderjährigen begründen kann, wenn diesem die Aussagebereitschaft fehlt. Der Anerkennung einer eigenen Entscheidungsbefugnis auch des unreifen Zeugen korrespondierte konsequenterweise eine eigene Pflicht zur Belehrung des Zeugen selbst ${ }^{72}$. In einer weiteren Entscheidung machte der Bundesgerichtshof noch einmal deutlich, daß der gesetzliche Vertreter nicht an Stelle des kindlichen Zeugen handelt ${ }^{\text {t3 }}$.

Die Änderung des $§ 52$ II StPO stellt sich nach alledem als Abschluß einer durch die Rechtsprechung begonnenen Entwicklung dar, in der immer stärker versucht wurde, die Interessen der Rechtsordnung an einer möglichst umfassenden Verwertung aller zugänglichen Beweismittel einerseits, die des Minderjährigenschutzes anderseits auszugleichen ${ }^{74}$ und dem Zweck des Zeugnisverweigerungsrechts gerecht zu werden.

Für den Zivilprozeß fehlt eine entsprechende Regelung noch und auch bei Zugrundelegung einer aus der Sicht des Zeugen vergleichbaren Konflikts- und Interessenlage erscheint es fraglich, ob der Rückgriff auf die strafprozessuale Regelung allein ausreichend ist, auch im Zivilprozeß zu einer befriedigenden Lösung zu gelangen. Zweifel ergeben sich zum einen daraus, daß die ZPO weitergehende Weigerungsmöglichkeiten einräumt ${ }^{75}$,

${ }^{69} B G H$ GS St. $12,235,239$ f v. 8.12.1958.

${ }^{70}$ BGHSt 12, 235, 242.

${ }^{71}$ BGHSt $14,159,160$ v. 2.3.1960.

${ }^{72}$ BGHSt 21, 303, 306 v. 19.9.67. Zur Belehrung im einzelnen s. unten $\S 7$.

${ }^{73}$ B GHSt 23, 221, 222 v. 27.1.1970.

${ }^{74} \mathrm{Zu}$ den sich gegenüberstehenden Aspekten Orlowsky, S. 5 ff.

${ }^{75}$ E. Peters, ZZP 77, 444, 449 f erklärt dies damit, daß dem Gesetzgeber die Wahrheitsfindung im Strafprozeß besonders vordringlich erschien. Im einzelnen unten $\S 6$. 
zum anderen an den unterschiedlichen Konkliktslagen der sonstigen Verfahrensbeteiligten und der für Straf- und Zivilprozeß jeweils unterschiedlichen Gewichtung der Verfahrensziele, die auch in differierenden Beweisverfahren Ausdruck findet ${ }^{76}$.

\section{Die Ausübung von Zeugniszwang gegenüber einem minderjährigen Zeugen}

\section{Ordnungsmittel gemäß § 390 I ZPO}

Bei unberechtigter Zeugnis- oder Eidesverweigerung durch den erschienenen ${ }^{77}$ Zeugen kommt gemäß $§ 390$ I ZPO die Auferlegung der dadurch verursachten Kosten sowie die Verhängung von Ordnungsgeld und -haft in Betracht. Unberechtigt ist die Zeugnisverweigerung, wenn der Zeuge überhaupt keinen oder keinen ernsthaften oder einen bereits rechtskräftig für unerheblich erklärten Grund angibt bzw. den Grund nicht glaubhaft macht ${ }^{78}$.

Die Verurteilung erfolgt, ohne daß es eines hierauf gerichteten Antrags bedarf, mithin von Amts wegen ${ }^{79}$. Im übrigen gilt für die erstmalige Verhängung von Ordnungsmitteln aufgrund $\S 390 \mathrm{I}$ ZPO dasselbe, wie für Maßnahmen nach $\S 380 \mathrm{ZPO}^{80}$.

\footnotetext{
${ }^{76}$ Die Ausübung des Zeugnisverweigerungsrecht ist wegen der besonderen Bedeutung des Problems in $\S 6$ gesondert dargestellt.

${ }^{77}$ Die Folgen des Nicht-Erscheinens sind ausschließlich in $\S 380 \mathrm{ZPO}$ geregelt; dazu oben $\S 4$ C. Zum Verhältnis von $\S 380 \mathrm{ZPO}$ und $\S 390 \mathrm{ZPO}$ Wieczorek, $\S 390 \mathrm{Anm}$. A I a.

${ }^{78}$ Baumbach-Lauterbach-Hartmann, $\S 390$ Anm. 2 A.

${ }^{79}$ Stein-Jonas-Schumann, § $390 \mathrm{Rn} 5$.

${ }^{80}$ Ebenda. Zu § $380 \mathrm{ZPO}$ oben $\S 4 \mathrm{C}$ II.
} 


\section{Die Erzwingungshaft nach § 390 II ZPO}

Nach allgemeiner Ansicht sind die genannten Ordnungsmittel von der Verhängung der Erzwingungshaft im Sinne des § 390 II ZPO zu unterscheiden. Der maßgebliche Differenzierungsgrund wird darin gesehen, da $\beta$ es sich bei der Erzwingungshaft nicht um eine Ordnungsstrafe mit echtem Strafcharakter handelt, sondern um ein Beugemittel ${ }^{81}$. Die Vorschriften der $\S \S 904 \mathrm{ff}$ ZPO sind auf die Beugehaft anwendbar.

Voraussetzung für die Anordnung der Zwangshaft ist neben der wiederholt unberechtigten Zeugnis- bzw. Eidesverweigerung ein Antrag der beweisführenden Partei ${ }^{82}$. Nicht notwendig ist, daß das verhängte Ordnungsmittel im Sinne des $§ 390$ I ZPO bereits vollstreckt wurde ${ }^{83}$.

\section{Die Verhängung gegen einen schuldunfähigen Zeugen}

Die Unterscheidung zwischen Ordnungs- und Zwangsmitteln gewinnt Bedeutung bei der Frage, ob sie auch gegen einen Minderjährigen ausgesprochen werden dürfen. Für Ordnungsmittel als Folge unentschuldigten Fernbleibens nach $\S 380 \mathrm{ZPO}$ ist dies zu verneinen, soweit der Minderjährige schuldunfähig oder nicht einsichtsfähig ist. Dies gilt für alle Ordnungsmittel, also auch solche nach § 390 I ZPO.

Die Verhängung der Erzwingungshaft gegen Jugendliche ist weder in der Literatur noch, soweit ersichtlich, in der Rechtsprechung angesprochen ${ }^{84}$.

\footnotetext{
${ }^{81}$ Stein-Jonas-Schumann, §390 Rn 11; Wieczorek, §390 Anm. B IV; Zöller-Stephan, § 390 Rn 7 spricht von Zwangsmaßregel; Pastor, S. 4 ff zu $\S 888,890 \mathrm{ZPO}$, wo die Unterscheidung in gleicher Weise getroffen wird. Ähnlich auch BVerfGE 43, 101, 105 (zu § 96 OWiG) wonach die Erzwingungshaft mit keinerlei Unwerturteil verbunden sei, sondern den Betroffenen vielmehr dazu mahnen solle, seine Pflicht zu erfüllen.

${ }^{82}$ Der Antrag kann unter den Voraussetzungen des § 399 ZPO auch von der gegnerischen Partei gestellt werden. In Entmündigungsverfahren ist ein Antrag stets entbehrlich; Baumbach-Lauterbach-Hartmann, § 390 Anm. 4; Stein-Jonas-Schumann, § 390 Rn 7 f.

${ }^{83}$ Thomas-Putzo, $\S 390$ Anm. 2.

${ }^{84}$ Soweit überhaupt Äußerungen im Zusammenhang mit Minderjährigen vorliegen, beziehen sie sich auf Ordnungsmittel.
} 
Allenfalls wird von der Unzweckmäßigkeit von Beugemitteln gegen Minderjährige gesprochen ${ }^{85}$.

Sowohl die Aussage als auch die Eidesleistung sind höchstpersönliche Handlungen; es bietet sich daher ein Vergleich mit § 888 ZPO an. Nach dieser Vorschrift wird ein Titel auf Vornahme einer nicht vertretbaren Handlung vollstreckt, indem der sich weigernde Schuldner zur Vornahme der Handlung durch Zwangsgeld und Zwangshaft angehalten wird ${ }^{86}$. Die Möglichkeit der Verhängung gegen einen minderjährigen Schuldner wird damit gerechtfertigt, die Erzwingungsmaßnahme beziehe sich auf die geschuldete Handlung, und diese sei keine Prozeßhandlung ${ }^{87}$.

Obgleich auch die vom Zeugen vorzunehmenden Handlungen keine Prozeßhandlungen sind ${ }^{88}$, kann eine Verhängung von Erzwingungshaft gegen einen Schuldunfähigen oder nicht Einsichtsfähigen nicht überzeugen. Sowohl die Ordnungs- als auch die Zwangsmittel sind eine Folge der Nichterfüllung gesetzlicher Zeugenpflichten. Ihre Verhängung ersetzt weder das Erscheinen noch die Aussage des Zeugen; sie sollen diesen vielmehr dazu veranlassen, seinen Zeugenpflichten noch nachzukommen. Gegenüber dieser Funktion der Sanktionsmittel kann die Unterscheidung von Ordnungs- und Zwangsmitteln anhand ihres Straf- oder Beugecharakters keine grundlegende Bedeutung erlangen ${ }^{89}$.

Für eine Gleichstellung bezüglich der Schuld- und Einsichtsfähigkeit spricht auch, daß nach $\$ 390$ II ZPO Voraussetzung für die Anordnung der Erzwingungshaft die vorherige Verhängung von Ordnungsmitteln ist. Letztere ist jedoch nur bei einsichtsfähigen Minderjährigen zulässig ${ }^{90}$.

\footnotetext{
${ }^{85}$ So etwa Lenckner, NJW 1965, 959, 960.

${ }^{86}$ Auch hier handelt es sich um reine Beugemaßnahmen ohne Strafcharakter, OLG Hamm, NJW-RR 1988, 1087, 1088; Baumbach-Lauterbach-Hartmann, § 888 Anm. 3 B a ("andersartige Rechtsnachteile"); Brehm, NJW 1976, 1730; Zöller-Stöber, § 888 Rn 7.

${ }^{87}$ In dieser Weise etwa Stein-Jonas-Münzberg, § $888 \mathrm{Rn} 43$, nach dessen Ansicht es im Rahmen des § 888 ZPO nicht auf Verschulden ankommt. Danach wäre die Verhängung auch gegen Schuldunfähige zulässig. Inkonsequent ist es aber, dennoch auf die Einsichtsfähigkeit in die Bedeutung des Zwangs- und Ordnungsmittels abzustellen, weil Schuldfähigkeit die Einsichtsfähigkeit voraussetzt; Thomas-Putzo, $\S 888$ Anm. $3 \mathrm{c}$ aa a.E.; Zöller-Stöber, ebenda (Fn 86).

${ }^{88}$ Dazu oben $\S 5$ B I 1.

${ }^{89}$ So wohl auch Grunsky, S. 25 zu § 888 ZPO.

${ }^{90}$ Oben $\S 5 \mathrm{C}$ I und $\S 4 \mathrm{C}$ I.
} 


\section{Zwangsmittel gegen die gesetzlichen Vertreter}

Nach hier vertretener Auffassung hat die Unterscheidung zwischen Ordnungs- und Zwangsmitteln keine Auswirkungen auf die Frage, ob sie gegen Minderjährige verhängt werden können. Dasselbe gilt im Zusammenhang mit der Frage, ob an Stelle des Zeugen gegen die gesetzlichen Vertreter Zeugniszwang ausgeübt werden darf. Mangels gesetzlicher Regelung fehlt die Rechtsgrundlage für Sanktionen gegen die Eltern ${ }^{91}$.

\section{Die Beschwerde gegen die Verhängung von Ordnungsmitteln}

Die Verhängung von Ordnungsmitteln ist durch den Zeugen gemäß § 390 III ZPO mit dem Rechtsmittel der Beschwerde anfechtbar. Die Besonderheiten, die sich aus der Minderjährigkeit des Zeugen und gegebenenfalls Beschwerdeführers ergeben sind im Zusammenhang mit den weiteren Rechtsschutzmöglichkeiten dargestellt ${ }^{92}$

\section{Ergebnis}

Die Zeugenpflicht besteht aus der Pflicht zum Erscheinen, zur Aussage und zur Eidesleistung. Es handelt um eine prozessuale Pflicht, der jeder Zeugnisfähige unterworfen ist.

Die Erscheinenspflicht schränkt das Aufenthaltsbestimmungsrecht der gesetzlichen Vertreter ein, ohne daß eigene Maßnahmen des Prozeß- oder des Vormundschaftsgerichts erforderlich wären. Die Aussagepflicht besteht bei einem Minderjährigen anders als die Eidespflicht unabhängig von seiner Einsichtsfähigkeit in ein Zeugnisverweigerungsrecht.

\footnotetext{
${ }^{91}$ Im einzelnen oben § 4 C III zur Verhängung von Ordnungsmitteln nach $\S 380$ ZPO gegen die gesetzlichen Vertreter.

${ }^{92}$ Zur Beschwerde s.u. § 10 B.
} 
Das Zeugnisverweigerungsrecht ist eine Art prozessuale Einrede des Zeugen. Mit seiner Qualifizierung als öffentlich-rechtliche Willenserklärung oder als Prozeßhandlung können keine besonderen Ausübungsvoraussetzungen verbunden werden. Eine gesetzliche Zeugenmündigkeit kennt die Zivilprozeßordnung nicht.

Die Ausübung von Zeugniszwang gegen schuldunfähige Minderjährige ist unzulässig. Schuldfähigkeit setzt die Vollendung des vierzehnten Lebensjahres und die Reife voraus, die Ordnungs- und Zwangsmaßnahmen als Folgen des eigenen pflichtwidrigen Verhaltens zu verstehen. Zwangsmaßnahmen gegen die gesetzlichen Vertreter entbehren der gesetzlichen Grundlage. 


\section{§ 6 Die Ausübung des Zeugnisverweigerungsrechts im Zivilprozeß durch einen Minderjährigen}

\section{A. Einsichtsfähigkeit als Ausübungsvoraussetzung}

Voraussetzung für die selbständige Entscheidung, als Zeuge aussagen zu wollen oder von einem Weigerungsrecht Gebrauch machen zu wollen, ist nach allgemeiner Überzeugung und der überwiegend strafgerichtlichen Rechtsprechung des Bundesgerichtshofs, daß der Minderjährige die Einsichtsfähigkeit in den Zweck des ihm zustehenden Zeugnisverweigerungsrechts besitzt. Eine früher vertretene Ansicht, wonach es auf die Einsichtsfähigkeit nicht ankomme, weil umgekehrt auch die Zeugenpflicht ohne diese Voraussetzung besteht ${ }^{1}$, ist heute unabhängig von der Prozeßart nach der dargestellten Rechtsprechung nicht mehr vertretbar.

Die herrschende Ansicht führt dazu, daß die Eltern im Fall gegebener Einsichtsfähigkeit von der Mitwirkung ausgeschlossen sind. Sie können also weder die Aussage verhindern oder erzwingen noch sind sie selbst über das Zeugnisverweigerungsrecht zu belehren. Das wirft die Frage auf, ob nicht umgekehrt das elterliche Sorgerecht gem. §§ 1626 ff BGB zu einer Beteiligung der gesetzlichen Vertreter zwingt ${ }^{2}$.

Der Konflikt zwischen der elterlichen Sorge und dem Selbstbestimmungsrecht des Kindes hat in der Literatur und der Rechtsprechung breiten Raum eingenommen ${ }^{3}$; im vorliegenden Zusammenhang sollen lediglich die Grundpositionen kurz dargelegt werden.

Ausgangspunkt muß das Selbstbestimmungsrecht des Minderjährigen sein, dem als eigenständige Rechtspersönlichkeit ein entsprechendes allge-

\footnotetext{
${ }^{1}$ E. Schmidt, JR 1959, 369, 370.

${ }^{2}$ Ähnlich BayObLG NJW 1967, 206, 208 = FamRZ 1966, 644, 646; Orlowsky, S. 73 ff.

${ }^{3}$ Gernhuber, Familienrecht, $\S 49$ VI; Moritz, S. 57 ff jeweils m.w.N.
} 
meines Persönlichkeitsrecht mit Grundrechtsrang zukommt ${ }^{4}$. Dieses Persönlichkeitsrecht, abgeleitet aus Art. 2 I GG ${ }^{5}$, umfaßt auch das Zeugnisverweigerungsrecht als subjektives Recht des Betroffenen ${ }^{6}$. Das Bundesverfassungsgericht und Teile der Literatur nehmen an, daß dem Selbstbestimmungsrecht des Kindes in der Person der Eltern ein Gegenrecht aus Art. 6 I GG, nämlich das Recht der elterlichen Sorge und Erziehung erwächst ${ }^{7}$ und daher eine Abwägung kollidierender Grundrechte unter Berücksichtigung des jeweiligen Schutzzweckes zu erfolgen hat ${ }^{8}$. Dagegen steht die Auffassung Gernhubers, das Persönlichkeitsrecht des Kindes enthalte die elterliche Gewalt, da diese die Entfaltung der Persönlichkeit in Lebensphasen sichere, die selbstverantwortliche Entscheidungen noch nicht zulassen ${ }^{9}$. Einer Entscheidung bedarf die Streitfrage hier nicht, denn die praktische Bedeutung der Auseinandersetzung ist gering. Maßgeblich ist die Übereinstimmung der verschiedenen Ansichten, wonach die Selbstbestimmung des Minderjährigen durch das Sorgerecht der Eltern nur eingeschränkt ist, solange und soweit dies nach dem Entwicklungsstand und zur Förderung des Wohls des Kindes geboten ist. Erlaubt der Entwicklungsstand des Minderjährigen eigenverantwortliche Entscheidungen, ist er dazu somit grundsätzlich berechtigt ${ }^{10}$. Sobald die Verstandesreife vorliegt, ein eigenes Recht $\mathrm{zu}$ verstehen und verantwortlich auszuüben, tritt daher, zumindest in personensorgerechtlichen Fragen ${ }^{11}$, die Entscheidungskompetenz der gesetzlichen Vertreter zurück ${ }^{12}$.

\footnotetext{
${ }^{4}$ Bosch, FamRZ 1959, 160 u. 203; Krüger, FamRZ 1956, 329, 333. Zur Fähigkeit Minderjähriger, Träger von Grundrechten zu sein und zur Grundrechtsmündigkeit als Ausübungsbeschränkung Hohm, NJW 1986, 3107, 3108 ff.

${ }^{5}$ Gernhuber, FamRZ 1962, 89, 91 ff.

${ }^{6}$ OLG Stuttgart, FamRZ 1965, 515; Erman-Michalski, § 1626 Rn 21.

${ }^{7}$ Dazu BVerfG DAV 1989, 149 ff.

${ }^{8}$ BVerfG, FamRZ 1986, 871, 874 = NJW 1986, 3129, 3130; Moritz, JA 1981, 186 f.

${ }^{9}$ Gernhuber, FamRZ 1962, 89, 92 f. Ebenso aus neuerer Zeit OLG Karlsruhe, NJW 1989, $2398 \mathrm{f}$.

${ }^{10}$ Dies ergibt sich schon aus $\S 1626$ II BGB, wonach die Eltern die wachsende Fähigkeit und das verantwortungsbewußte Handeln des Kindes berücksichten und fördern sollen; es handelt sich eine immanente, nicht dem Verhältnismäßigkeitsgrundsatz unterliegende Einschränkung des elterlichen Sorgerechts.

${ }^{11} B G H Z$ 29, 33, 36 f; ähnlich BVerfGE 72, 155, 170 ff; MünchKomm-Hinz, § 1629 Rn 1a.

${ }^{12} \mathrm{Im}$ Ergebnis ähnlich entscheidet die h.M. im Strafrecht zur Frage, ob und unter welchen Voraussetzungen ein Jugendlicher wirksam auf ein strafrechtlich geschütztes Rechtsgut verzichten kann. Ein Verzicht kann selbständig geäußert werden, wenn der Betroffene die aus-
} 
Bezogen auf das Zeugnisverweigerungsrecht soll die erforderliche Einsichtsfähigkeit vorliegen, wenn der Minderjährige aufgrund seiner geistigen und intellektuellen Fähigkeiten und Entwicklung zu erkennen in der Lage ist, daß der angeklagte Angehörige etwas Unrechtes getan hat, daß ihm dafür Strafe droht und daß die eigene Aussage möglicherweise zu der Bestrafung beitragen kann ${ }^{13}$. Für den Bereich des Zivilprozesses dürfte die so verstandene, an strafprozessualen Erfordernissen orientierte Definition der Einsichtsfähigkeit jedoch nicht genügen, da die potentiellen Konfliktslagen, derentwegen die ZPO ein Zeugnisverweigerungsrecht einräumt, nicht nur in den auch gefühlsmäßig zu erfassenden Kategorien von Recht und Unrecht zu erfassen sind. Dies beruht zum einen an der praktisch unbegrenzten Zahl der denkbaren Interessenskonflikte, die im Einzelfall hinter einem Rechtsstreit stehen mögen, zum anderen an den der StPO unbekannten Zeugnisverweigerungsrechten wie z.B. dem des $\S 384$ Ziff. 1 ZPO, der den Zeugen vor der Gefahr vermögensrechtlicher Schäden durch die Erfüllung der Aussagepflicht bewahren will ${ }^{14}$. Nachdem das Postulat hinreichender Einsichtsfähigkeit für die selbständige Ausübung des Weigerungsrechts als Sicherung einer verantwortungsbewußten Entscheidung gedacht ist ${ }^{15}$, sollte es für den Zivilproze $ß$ genügen, daß der Minderjährige im Einzelfall versteht, daß seine Aussage ihm selbst oder einem Angehörigen zum Nachteil geraten kann und die Prozeßordnung ihn daher von seiner Zeugenpflicht freistellt, sofern er dies zur Vermeidung eines Gewissenskonfliktes wünscht ${ }^{16}$. Die Konsequenz dieser Ansicht ist allerdings, daß die Frage der Einsichtsfähigkeit nicht abstrakt, sondern im Einzelfall und gerade im Hinblick auf den geltend gemachten Grund der Weigerung zu überprüfen ist, da es ohne weiteres denkbar ist, daß der Minderjährige zwar die Konfliktsituation durch Belastung eines engsten Familienangehörigen durch seine Aussage versteht, nicht aber die drohende Gefahr eines unmittelbaren Vermögensschadens zu begreifen vermag. Kann die Verstandesreife nicht soweit festgestellt werden, daß sie zur Überzeugung des Gerichts vorliegt, ist im Zwei-

reichende Verstandesreife besitzt, die Folgen zu übersehen. Zur Begründung wird auf das Selbstbestimmungsrecht des Kindes verwiesen; dazu Lesch, NJW 1989, 2309, 2310 ff m.w.N.

${ }^{13}$ BGHSt 12, 235, $239 \mathrm{f} ; 14,159,161 \mathrm{f}$.

${ }^{14} \mathrm{Zu} \S 384$ Ziff. 1 ZPO Stein-Jonas-Schumann, § $384 \mathrm{Rn} 3 \mathrm{f}$.

${ }^{15}$ Orlowsky, S. 53 (zur StPO).

${ }^{16}$ OLG Stuttgart, FamRZ 1965, 515, 517. 
fel von der Unreife des Zeugen auszugehen ${ }^{17}$. Die Beteiligung der Eltern kann dann zwar im Einzelfall einen Eingriff in das Selbstbestimmungsrecht des Kindes darstellen, doch ist dies gerade im Interesse des Minderjährigen eher hinzunehmen, als der unwirksame Verzicht auf das Zeugnisverweigerungsrecht mit seinen unabsehbaren Folgen im umgekehrten Fall.

Die Feststellung der geistigen Reife ist Aufgabe des erkennenden Gerichts, das die Beweisaufnahme und damit auch die Zeugenvernehmung durchführt ${ }^{18}$. Dabei muß die Verstandesreife positiv festgestellt werden ${ }^{19}$, weil sich das Gericht selbst von den das Verfahren und dessen Zulässigkeit betreffenden Voraussetzungen überzeugen muß ${ }^{20}$. Eine Übertragung der Entscheidung dieser Teilfrage auf das Vormundschaftsgericht kommt daher nicht in Betracht ${ }^{21}$.

Feste Altersgrenzen, an Hand derer eine bestimmte Verständnisreife zumindest zu vermuten wäre und die den Vorteil zusätzlicher Rechtssicherheit für alle Beteiligten hätte, werden somit de lege lata weder von der Rechtsprechung noch von der Literatur ${ }^{22}$ anerkannt; die Anforderungen werden von der Rechtsprechung nicht allzu hoch angesetzt ${ }^{23}$, auch wenn vielfach im Schriftum der Hinweis zu finden ist, die Altersgrenze von 14 Jahren sei als Indiz für ausreichende Reife brauchbar ${ }^{24}$, ohne allerdings nach dem geltend gemachten Weigerungsgrund zu differenzieren.

\footnotetext{
${ }^{17}$ OLG Stuttgart, ebenda (Fn 16); Schimansky, FS f. Pfeiffer, S. 297, 305.

${ }^{18}$ BayObLG, NJW 1967, 206, 209 = FamRZ 1966, 644, 647; Schneider, JurBüro 1970, $1021 \mathrm{f}$. Zum Strafverfahren RGSt 33, 393, 394; BGHSt 14, 159, 160. Im Strafprozeß kann die Prüfung auch während der Vernehmung durch die Staatsanwaltschaft oder der Polizei erfolgen, OLG Stuttgart, OLGZ 1985, 385, 386 = FamRZ 1985, 1154, 1155.
}

${ }^{19}$ So RGSt 47, 297 zur Eidespflicht nach $\S 60$ Ziff. 1 StPO. Auch im Zivilprozeß ist eine exakte Beurteilung notwendig, weil durch die Nicht-Hinzuziehung der gesetzlichen Vertreter eine Gefährdung des Zeugen nicht ausgeschlossen werden kann. Umgekehrt kann bei irrtümlicher Verneinung der Verstandesreife durch die Zustimmungsverweigerung der Eltern das Recht der Parteien auf Beweis verletzt werden; dazu auch unten $\$ 9 \mathrm{C}$ III.

${ }^{20}$ BayObLG NJW 1967, 206, 209 = FamRZ 1966, 644, 647.

${ }^{21}$ BayObLG, ebenda (Fn 20). Zum vormundschaftsgerichtlichen Verfahren unten $\S 11$.

${ }^{22}$ Mit Ausnahme von Bosch, S. 47 (14 Jahre) und Staudinger-Donau, § 1626 Rn 75 (16 Jahre); dazu oben $\S 5$ B II 1.

${ }^{23}$ Das läßt sich allerdings nur aus der Zulassung auch sehr junger Zeugen schließen; beispielhaft $R G$ GA 59, 131, das auch bei Zeuginnen im Alter von $31 / 2$ und $41 / 2$ Jahren erst nach der Vernehmung prüfen will, ob ausreichende Verständisfähigkeit vorliegt. Ähnlich auch Bosch, S. 47 Fn 140. 


\section{B. Minderjährige mit ausreichender Verstandesreife}

Hat der minderjährige Zeuge die Fähigkeit bereits in ausreichendem Maße entwickelt, seine Zeugenpflichten sowie ein etwaiges Weigerungsrecht zu verstehen, d.h. die sich dahinter verbergende und je nach geltend gemachtem Weigerungsgrund differierende Konfliktsituation zu begreifen und in verantwortlicher Weise zu lösen, ist er nach ganz überwiegender Auffassung einem bereits volljährigem Zeugen gleichzustellen ${ }^{25}$. Der Umstand der Minderjährigkeit hat danach keine Auswirkung auf die prozessuale Stellung des Zeugen. Der Minderjährige entscheidet daher allein über die Ausübung eines Zeugenisverweigerungsrechts; die gesetzlichen Vertreter sind weder an der Belehrung noch an der Willensbildung beteiligt.

Für den Strafprozeß wurde vor dem Inkrafttreten des $\S 52$ II StPO in der heutigen Fassung eine abweichende Meinung vertreten, wonach auch in diesem Fall die Zustimmung der gesetzlichen Vertreter erforderlich $\operatorname{se}^{26}$. Die Aussagebereitschaft des Minderjährigen sollte nach dieser Auffassung allein nicht ausreichen, da aus Gründen des Minderjährigenschutzes die elterliche Sorge, die der Fürsorge zum Wohl des Kindes diene, keine Einschränkung erlaube, mithin eine Beteiligung der Eltern auch hier geboten sei. Zur Begründung wurde ausgeführt, daß der Minderjährige im Zweifel nicht in der Lage sei, den Interessenkonflikt wirklich zu überschauen ${ }^{27}$. Darüberhinaus müsse die elterliche Sorge als Regulativ der möglichen Unsicherheiten bei der Feststellung der geistigen Reife des Zeugen dienen ${ }^{28}$.

Für den Zivilprozeß wird eine Beteiligung der Eltern auch beim einsichtsfähigen Jugendlichen nur noch insoweit erwogen, als es sich um das Zeugnisverweigerungsrecht nach § $384 \mathrm{Nr} .1$ ZPO handelt, da hier keine persönlichen, sondern nur vermögensrechtliche Erwägungen für die Aussagever-

\footnotetext{
${ }^{24}$ OLG Stuttgart, FamRZ 1965, 515, 517; Grundmann, S. 92; MünchKomm-Hinz, § 1626 Rn 46. Weiter Schneider, JurBüro 1970, 1021, der bis zum achten Lebensjahr fehlende Reife annimmt.

${ }^{25}$ Erman-Michalski, § 1626 Rn 21; Stein-Jonas-Schumann, § 383 Rn 5.

${ }^{26}$ Orlowsky, S. $73 \mathrm{ff}$.

${ }^{27}$ Orlowsky, S. 75 ff. Ähnlich für den Zivilprozeß Bruns, Rn 185 d; derselbe, ZZP 77, 318.

${ }^{28}$ Orlowsky, S. 78.
} 
weigerung maßgeblich seien und diese der Vermögenssorge zuzurechnen seien, für die die gesetzliche Vertreter offenbar uneingeschränkt zuständig sein sollen ${ }^{29}$.

Diese Ansichten dürften jedoch heute nicht mehr vertretbar $\operatorname{sein}^{30}$ und sind im übrigen auch nicht sachgerecht, da sie inkonsequent die zunächst bejahte Einsichtsfähigkeit wieder in Frage stellen, weil die Folgen einer Aussage bei gegebenem Weigerungsgrund unübersehbar $\operatorname{sind}^{31}$. Darüberhinaus bleibt die Frage unbeantwortet, wodurch die Einschränkung des Selbstbestimmungsrechts noch gerechtfertigt ist, wenn mangels Schutzbedürftigkeit der Grund der Einschaltung der gesetzlichen Vertreter entfallen ist. Schwerer wiegt die Befürwortung der Beteiligung der Eltern bei dem Weigerungsrecht aus § $384 \mathrm{Nr} .1 \mathrm{ZPO}$ mit der genannten Begründung, doch spricht dagegen, daß nicht die Wahrung von Vermögensinteressen im Vordergrund steht, sondern die Vermeidung eines Gewissenskonfliktes. Die Entscheidung über die Ausübung eines Zeugnisverweigerungsrechtes ist daher unabhängig vom Weigerungsgrund immer eine personenrechtliche Frage $^{32}$, so daß das Argument letztlich nicht greift. Auch die angeführten praktischen Erwägungen zwingen m.E. nicht zu einer anderen Beurteilung; konzediert man praktische Schwierigkeiten bei der Prüfung der Verständnisreife eines jugendlichen Zeugen, sollte man diese durch eine generelle Regelung der Zeugenmündigkeit lösen, nicht aber durch eine vom Einzelfall abhängige Beteiligung der Eltern. Von daher erscheint die von der Praxis durchgeführte Gleichstellung des Minderjährigen mit einem Volljährigen als sachgerechtere Lösung.

\footnotetext{
${ }^{29}$ Staudinger-Donau, § 1626 Rn 75 a.E.. Ähnlich Bosch, S. 48 f.

${ }^{30}$ Ebenso für die StPO Löwe-Rosenberg-Dahs, § $52 \mathrm{Rn} 22$.

${ }^{31}$ So aber Orlowsky, S. 76.

${ }^{32}$ OLG Stuttgart, NJW 1971, 2237, 2238; MünchKomm-Hinz, § 1626 Rn 47; Schaub, FamRZ 1966, 134, 135.
} 


\section{Minderjährige ohne ausreichende Verstandesreife}

\section{Wegfall des Zeugnisverweigerungsrechts wegen fehlendem Konfliktsempfinden?}

Es ist der Sinn des Zeugnisverweigerungsrechts, den Zeugen vor einem Gewissenskonflikt zwischen Aussage und Verletzung eigener, als schutzwürdig anerkannter Interessen, sei es der Schutz der Familienbande im Fall des $\$ 383$ I Nr. 3 ZPO, seien es vermögensrechtliche Interessen wie bei $\S 384 \mathrm{Nr} .1 \mathrm{ZPO}$ zu bewahren. Daher erscheint es zunächst naheliegend, die Ausübung des Weigerungsrechts davon abhängig zu machen, daß der Gewissenskonflikt subjektiv auch als solcher empfunden wird ${ }^{33}$.

Soweit eine derartige Einschränkung früher erörtert wurde, ist sie heute nicht mehr vertretbar, da sie einseitig auf das Zeugnisverweigerungsrecht als Einrede im ausschließlichem Interesse des - bei fehlendem Konfliktsempfinden nicht schutzbedürftigen - Zeugen abstellt ${ }^{34}$. Diese Ansicht würde zum einen auf Bedenken stoßen, weil die bloße Unkenntnis oder auch die Unfähigkeit zur Kenntnisnahme ebensowenig Anlaß bietet, das gesetzlich normierte Recht entfallen zu lassen, wie sachfremde Erwägungen bei der Entscheidung über die Wahrnehmung des Rechts. Darüberhinaus muß berücksichtigt werden, daß die Zeugnisverweigerungsrechte auch objektive Zwecke neben dem Schutz des Zeugen verfolgen, nämlich die Vermeidung einer von vornherein mit geringem Beweiswert belasteten Aussage ${ }^{35}$. Es besteht ein öffentliches Interesse daran, nur solche Beweispersonen unbedingt

\footnotetext{
${ }^{33} \mathrm{Zu}$ der weitergehenden Ansicht, nach der von der Verstandesreife nicht nur die selbständige Zeugnisverweigerung, sondern auch das Bestehen der Aussagepflicht abhängt oben § 5 A II 2.

${ }^{34}$ Z.B. Schimmack, JW 1924, 1667 f. Bruns, Rn 185 d, will wegen des fehlenden inneren Konfliktes nur auf die gesetzlichen Vertreter abstellen.

${ }^{35}$ BayObLG, NJW 1967, 206, 209 = FamRZ 1966, 644, 647; Hahn, Materialien I, S. 312; Hampel, FamRZ 1974, 53, 54 f; Stein-Jonas-Schumann, § 383 Rn 1. E. Peters, ZZP 76, 145, 161 weist unter Bezug auf die Motive darauf hin, daß der Zweck der Zeugnisverweigerungsrechte auch der Schutz der Rechtspflege vor falschen Zeugnissen ist. Zum Strafprozeß Orlowsky, S. 46; BGHSt. 12, 235, 239. A.A. KMR-Paulus, Vorbem. $\$ 48$ Rn 82 m.w.N.; BGHSt GS $11,213,216$ zu $§ 52$ II StPO.
} 
zur Wahrheitsfindung heranzuziehen, die nicht aufgrund typischer, d.h. einzelfallunabhängiger Konfliktsituationen die Gefahr erheblicher Voreingenommenheit in sich bergen. Hinzu treten weitere objektive Schutzzwecke neben den Genannten, was insbesondere bei dem Zeugnisverweigerungsrecht zum Schutz von Berufsgeheimnissen deutlich wird. Die Möglichkeit der Aussageverweigerung soll hier auch das als sozial wichtig anerkannte Vertrauensverhältnis zwischen Weigerungsberechtigtem und Dritten, etwa Arzt und Patient, schützen ${ }^{36}$.

Die objektiven Seiten des Zeugnisverweigerungsrechts würden vernachlässigt, wollte man ihre Ausübung zusätzlich von einem Konfliktsempfinden oder anderen subjektiven Voraussetzungen abhängig machen, zumal diese Voraussetzungen dann nicht ohne Grund auf Minderjährige beschränkt werden könnten.

Festzuhalten bleibt somit, daß auch derjenige grundsätzlich zeugnisverweigerungsberechtigt ist, der nicht die notwendige Einsichtsfähigkeit besitzt, zu verstehen, weshalb er dies darf. Zu klären bleibt, wie das Recht in diesen Fällen ausgeübt wird. Im Folgenden wird danach zu unterscheiden sein, ob die gesetzlichen Vertreter, im Regelfall die Eltern, an dem Verfahren, in dem der Minderjährige als Zeuge aussagen soll, selbst als Partei beteiligt sind oder nicht, da sich danach die Frage bemißt, wem gegebenenfalls das Recht zur Vertretung zusteht.

${ }^{36} B G H$, NJW 1964, 449, 450 zu $\S 383$ I Nr. 5 a.F., jetzt Nr. 6. Lenckner, NJW 1965, 321, 322. Ähnlich E. Peters, ZZP 76, 145, 162; Rupp, Gutachten, S. 199 f; Habscheid, ZZP 96, 306, 317 ff begründet die Zeugnisverweigerungsrechte ebenfalls auch objektiv als Ausprägungen verfassungsrechtlicher Wertentscheidungen. 


\section{Beteiligung keines Elternteils am Verfahren}

\section{Zeugnisverweigenung und Zustimmungserfordernis}

\section{a) Personensorgerecht und Höchstpersönlichkeit der Zeugnisverweigerung}

Soll der Minderjährige in einem Prozeß als Zeuge aussagen, in dem keiner seiner Eltern als gemeinsame gesetzliche Vertreter beteiligt sind, entspricht es allgemeiner Meinung, die Entscheidung über die Ausübung des Zeugnisverweigerungsrechtes den Eltern zuzuweisen ${ }^{37}$. Grundlage dieser Zuweisung ist die elterliche Sorge im Sinne des §§ 1626, 1629 I BGB, wonach beide Elternteile das Kind gemeinsam vertreten. Im folgenden wird von diesem Regelfall ausgegangen; ist nur ein Elternteil sorgeberechtigt, so entscheidet er allein.

Die Zulässigkeit der Vertretung begegnet Bedenken weder im Hinblick auf die materiell-rechtliche Seite der Ausübung des Zeugnisverweigerungsrechtes durch öffentlich-rechtliche Willenserklärung, noch im Hinblick auf ihre Einordnung als Prozeßhandlung ${ }^{38}$. Fraglich ist aber, ob nicht der höchstpersönliche Charakter der Gewissensentscheidung ${ }^{39}$ einer Übertragung auf einen Vertreter entgegen steht.

Einen allgemeinen Rechtssatz des Inhaltes, bei höchstpersönlichen Geschäften sei Vertretung grundsätzlich unzulässig, gibt es nicht ${ }^{40}$. Dement-

\footnotetext{
${ }^{37}$ OLG Stuttgart, NJW 1971, 2237, 2238; Stein-Jonas-Schumann, § 383 Rn 7.

${ }^{38}$ BayObLG, NJW 1967, 206, 208 = FamRZ 1966, 644, 646; Bosch, S. 39; E. Schmidt, JR 1959, 369, 370. Dazu auch oben § 5 B I 1.

${ }^{39}$ Bosch, S. 50 f; Hampel, FamRZ 1964, 125, 128. K. Peters, Gutachten, S. 115 f zu $\$ 52$ StPO.

${ }^{40}$ Soergel-Leptien, Vor $\S 164 \mathrm{Rn} 89,93$; a.A. Flume, $\S 13,10$. Flume hält die Vornahme höchstpersönlicher Rechtsgechäfte durch den gesetzlichen Vertreter bei Geschäftsunfähigen für ausgeschlossen, während der beschränkt Geschäftsfähige in Einzelfällen eine wirksame rechtsgeschäftliche Erklärung abgeben könne, wenn die gesetzlichen Vertreter zustimmen. Zumindest für das Prozeßrecht kann dieser Ansicht - auch unter Berücksichtigung des Umstandes, daß es hier auf Geschäftsfähigkeit nicht ankommt - nicht gefolgt werden. Es ist nicht einzusehen, dem geschäftsunfähigen Minderjährigen das Recht zur Zeugnisverweigerung ganz zu nehmen, weil es sich um eine höchstpersönliche Handlung handelt; der Gedanke des Minderjährigenschutzes würde dadurch geradezu ins Gegenteil verkehrt. Im Ergebnis ebenso BayObLG, NJW 1967, 206, 208 f = FamRZ 1966, 644, 646.
} 
sprechend wird §52 II 2 StPO analog auf den Zivilprozeß angewendet, so daß der nicht einsichtsfähige Minderjährige nur mit Zustimmung seiner gesetzlichen Vertreter aussagen darf, aber nicht muß $\beta^{41}$. Diese Vorschrift wird als Ausdruck eines allgemeinen Rechtsgedankens angesehen, der von der Verfahrensart unabhängig ist ${ }^{42}$. Grundsätzlich wird damit die Entscheidungsbefugnis über die Ausübung des Zeugnisverweigerungsrechtes in die Hand der gesetzlichen Vertreter gelegt; um jedoch bei fehlender Aussagebereitschaft des Minderjährigen nicht zu einem Aussagezwang zu kommen, wird auch dem nicht einsichtsfähigem Jugendlichen das Recht zugestanden, trotz Zustimmung der gesetzlichen Vertreter die Aussage zu verweigern. Begründet wird dies mit der Unzulässigkeit von Beugemitteln gegenüber Minderjährigen ${ }^{43}$, dem geringen Beweiswert einer erzwungenen Aussage ${ }^{44}$, mit der Notwendigkeit, einen Gewissenskonflikt des Minderjährigen zu verhindern ${ }^{45}$ oder - allgemeiner - mit dem Persönlichkeitsrecht des Minderjährigen, dessen Schutz es verbiete, gegen seinen Willen zur Aussage gezwungen zu werden ${ }^{46}$.

Im Ergebnis ist diesen Auffassungen zuzustimmen, da sie der Schutzbedürftigkeit des Minderjährigen und dem Grundsatz vom Vorrang des Kindeswohls gegenüber den Interessen der Eltern ${ }^{47}$ in wohl ausreichendem Maße Rechnung tragen. Allerdings scheint die Annahme, der Minderjährige werde bei fehlender Einsichtsfähigkeit von den Eltern vertreten, dem Zeugnisverweigerungsrecht als Gewissensentscheidung nicht gerecht zu werden ${ }^{48}$. Überzeugender ist es m.E. von der Unvertretbarkeit einer Gewissensentscheidung auszugehen ${ }^{49}$ und damit die Entscheidungsbefugnis grund-

\footnotetext{
${ }^{41}$ BayObLG, ebenda (Fn 40); Baumbach-Lauterbach-Hartmann, Einf. vor $\S 383$ - 391 Rn 3; Bruns, Rn 185 d; Erman-Michalski, § 1626 Rn 20; Grundmann, S. 91; MünchKomm-Hinz, § 1626 Rn 46; RGRK-Wenz, §1626 Rn 20; Stein-Jonas-Schumann, § 383 Rn 6 f; Wieczorek, $\S 383$ Anm. A IV a; Zöller-Stephan, § $383 \mathrm{Rn} 4$.

${ }^{42}$ Soergel-Strätz, § $1629 \mathrm{Rn} 18$.

${ }^{43}$ So Lenckner, NJW 1965, 959, 960; OLG Stuttgart, FamRZ 1965, 515, 516.

${ }^{44}$ Grundmann, S. 93; Erman-Michalski, § 1626 Rn 20; OLG Stuttgart, FamRZ 1965, 517.

${ }^{45}$ Stein-Jonas-Schumann, § 383 Rn 7; Orlowsky, S. 70 (zur StPO vor 1974).

${ }^{46}$ Erman-Michalski, § 1626 Rn 21; Staudinger-Donau, § 1626 Rn 75.

${ }^{47}$ BVerfGE 61, 358, 378 = FamRZ 1982, 1179, 1181; FamRZ 1986, 871, $874=$ NJW 1986, 3129,3130 .

${ }^{48}$ Zweifelnd auch Bosch, S. 50 f; Hanack, JZ 1971, 126; Weber, MDR 1962, 169 ff.

${ }^{49}$ Hampel, FamRZ 1964, 125, 128; ähnlich Soergel-Strätz, § 1629 Rn 22.
} 
sätzlich dem Minderjährigen selbst zuzusprechen ${ }^{50}$. Nur aus Gründen der Schutzbedürftigkeit des Rechtsträgers, der eine Gewissensentscheidung möglicherweise gerade nicht zu treffen vermag, tritt neben die eigenständige Entscheidung des Zeugen, auch wenn sie auf sachfremden Erwägungen beruht, das Erfordernis der Zustimmung der gesetzlichen Vertreter hinzu. Das Zustimmungsrecht der Eltern ergibt sich daher nicht aus § 1629 I BGB, sondern ist Ausfluß des Personensorgerechts aus $\S 1626$ I BGB $^{51}$.

b) Aussagebereitschaft als Voraussetzung der Zustimmung der gesetzlichen Vertreter

Bei fehlender Aussagebereitschaft braucht die Zustimmung der Eltern nicht eingeholt zu werden, da es dann an der Schutzbedürftigkeit des Kindes fehlt, das nur vor den unbedachten Folgen einer Aussage, nicht aber vor den Folgen einer Aussageverweigerung geschützt werden soll. Das bedeutet im übrigen zugleich, daß erst die Aussagebereitschaft des Minderjährigen festzustellen ist, bevor die gesetzlichen Vertreter gefragt werden ${ }^{52}$. Damit wird auch die Gefahr gemindert, daß der Minderjährige meint, schon allein aufgrund der Zustimmung seiner Eltern zur Aussage verpflichtet zu sein, eine Gefahr, der durch die eigene Belehrung auch gerade über diesen Punkt ${ }^{53}$ sonst nur unzureichend entgegen getreten würde.

\footnotetext{
${ }^{50}$ Ebenso Gernhuber, FamRZ 1962, 89, 95 Fn 48 zu $§ 52$ StPO a.F.; Stein-Jonas-Schumann, $\S 383$ Rn 9 Fn 9; OLG Düsseldorf, FamRZ 1973, 547 f. Für das Strafverfahren hat der BGH ebenfalls angenommen, ein Amtsvormund handle insoweit nicht an Stelle des Zeugen, BGHSt 23, 221, 222. Der BGH gab damit seine in BGHSt 12, 235, 242 geäußerte Rechtsansicht auf.

${ }^{51}$ LG Mannheim, FamRZ 1974, 158; Schaub, FamRZ 1966, 134, 135 nimmt ähnlich an, daß die Vertretung des Minderjährigen "im Willen" in den Bereich der Vertretung in persönlichen Angelegenheiten handelt.

${ }^{52}$ Grundmann, S. 93. Entsprechend bei der Bestellung eines Ergänzungspflegers; dazu unten $\S 11 \mathrm{ff}$.
}

${ }^{53}$ BGHSt 21, 303, 306. Zur Belehrung vgl. unten $§ 7$. 
c) Zeitpunkt der Zustimmung

Der Schutz des Minderjährigen gebietet, die Vernehmung erst vorzunehmen, wenn zum die Notwendigkeit der Zustimmung durch die gesetzlichen Vertreter festgestellt ist, die sich aus fehlender Einsichtsfähigkeit und gleichzeitiger Aussagebereitschaft ergibt und die Zustimmung auch erklärt wurde. Eine nur nachträgliche Beteiligung der Eltern erspart dem Jugendlichen im Zweifel nicht eine prozeßrechtswidrige Vernehmung bei nachträglicher Zustimmungsverweigerung. Die nachträgliche Beteiligung kommt daher nur in Betracht, wenn das Gericht die Notwendigkeit der Beteiligung anfänglich verkannt hat. In diesem Fall muß der Minderjährige aber noch aussagebereit sein oder an seiner bereits geleisteten Aussage festzuhalten bereit $\operatorname{sein}^{54}$.

Ungeklärt ist aber, ob die gesetzlichen Vertreter nach einer zu Unrecht erfolgten Vernehmung des Minderjährigen nachträglich die Zustimmung verweigern und die Aussage damit unverwertbar machen können.

Zunächst ist bei der unterbliebenen Einholung der Zustimmung der gesetzlichen Vertreter fraglich, ob nach erstatteter Aussage durch den Minderjährigen überhaupt noch ein Bedürfnis für die Beteiligung der Eltern besteht. Dies ist jedoch zu bejahen, da die Beteiligung der Eltern dem Schutz des Minderjährigen vor für ihn nicht absehbaren Folgen seiner Aussage dient und die Schutzbedürftigkeit nach der Aussage nicht entfallen ist, wenn die Aussage nach einer Zustimmungsverweigerung nicht mehr verwertet werden darf. Von einem derartigen Verwertungsverbot ${ }^{55}$ ist auszugehen, da im Falle des § 383 I Nr. 3 ZPO die Belastung der engen familiären Beziehung nicht schon mit der Aussage, sondern erst mit deren Verwertung im Verfahren und im Urteil vollendet ist ${ }^{56}$. Aus demselben Grund wird man für die Aussage des Minderjährigen das Vorliegen einer eindeutigen Zustimmung durch die gesetzlichen Vertreter auch noch nach der Aussage verlangen müssen, eine bloße Duldung genügt m.E. nicht ${ }^{57}$.

\footnotetext{
${ }^{54}$ So auch $B G H S t$ 23, 221, $223 \mathrm{f}$, der als weiteres Argument anführt, der Jugendliche könne nicht darüber belehrt werden, daß er trotz Zustimmung der gesetzlichen Vertreter nicht aussagen müsse, wenn die Zustimmung zur Zeit der Aussage noch gar nicht eingeholt ist. Ähnlich Schimansky, FS f. Pfeiffer, S. 297, 305.

${ }^{55}$ Dazu allgemein unter $\S 9$.

${ }^{56}$ Ebenso Habscheid, ZZP 96, 306, $320 \mathrm{f}$.

${ }^{57} \mathrm{Im}$ Strafverfahren wird die nachträgliche Genehmigung der Aussage durch die gesetzlichen
} 
Die Verweigerung der Zustimmung kommt nach dem Gesagtem nur bei Aussagebereitschaft des Minderjährigen in Betracht. Die Zeugnisverweigerung ist keine Prozeßhandlung ${ }^{58}$. Auch die Zustimmungsverweigerung ist wegen ihrer Nähe zur Zeugnisverweigerung nicht als Prozeßhandlung im engeren Sinne zu qualifizieren. Obwohl daher keine Prozeßhandlung der Eltern vorliegt, kommen die bürgerlichrechtlichen Vorschriften über Einwilligung und Genehmigung nicht zur Anwendung ${ }^{59}$. Der Fall der nachträglichen Zustimmungsverweigerung ist einer nachträglichen Zeugnisverweigerung entsprechend zu behandeln, da hier wie dort eine Abwägung der Interessen durch den bzw. die Ausübungsbefugten gegen eine Aussage stattgefunden hat und diese Entscheidung zu respektieren ist.

Das Zeugnisverweigerungsrecht kann bis zum Ende der Vernehmung ausgeübt werden ${ }^{60}$, bis zu diesem Zeitpunkt kann daher auch nachträglich die Zustimmung verweigert werden, sofern die gesetzlichen Vertreter selbst über ihr Recht belehrt wurden. Eine erst nach Abschluß der Vernehmung erteilte Zustimmung vermag dagegen keine Wirkung mehr zu entfalten. Der Verzicht auf ein Zeugnisverweigerungsrecht ist innerhalb der mündlichen Verhandlung und unter unmittelbarem Eindruck der Konfliktsituation zu erklären $^{61}$. Dasselbe hat für die Erteilung des Einverständnisses mit der Aussage zu gelten, weil sie rechtlich dieselbe Wirkung wie der mit der Aussagebereitschaft einhergehende Verzicht auf das Weigerungsrecht hat.

\footnotetext{
Vertreter ebenfalls für zulässig erachtet, Kleinknecht/Meyer, § 52 Rn 19; KMR-Paulus, § 52 Rn 23; Löwe-Rosenberg-Dahs, $\$ 52 \mathrm{Rn} 26$. Dagegen verweist der BGH darauf, daß der Zeuge nicht darüber belehrt werden könne, daß er trotz der Zustimmung der gesetzlichen Vertreter nicht auszusagen brauche, wenn diese ihre Zustimmung noch gar nicht erklärt haben, BGHSt 23, 221, 222 f. Dies kann nicht überzeugen, zumal sich die Zustimmungsbedürftigkeit unter Umständen erst während der Vernehmung und damit nach der Belehrung des Minderjährigen herausstellen kann. In einem derartigen Fall ist auch im Strafprozeß von der Verwertbarkeit der Aussage auszugehen, wenn die Eltern nachträglich zustimmen und der Zeuge an seiner Aussage festhält.

${ }^{58}$ Zur Gegenansicht vgl. oben § 5 B I 1.

${ }^{59}$ Soergel-Leptien, Vor $\S 182 \mathrm{Rn} 17$, der aber in Einzelfällen eine Anwendung für möglich hält.
}

${ }^{60}$ Stein-Jonas-Schumann, § $383 \mathrm{Rn} 19$; Thomas-Putzo, § 383 Anm. 1; Wieczorek, §383 Anm. B I.

${ }^{61}$ K. Peters, Gutachten, S. 121 zum Strafverfahren. 


\section{Zustimmungsbefugnis bei Meinungsverschiedenheiten der Eltern}

a) Entscheidung des Vormundschaftsgerichts gemäß § 1628 BGB?

Soweit beide Eltern das Sorgerecht gemeinsam inne haben ${ }^{62}$, üben sie die Zustimmungsbefugnis zur Aussage des nicht einsichtsfähigen Minderjährigen gemeinsam aus. Allerdings stellt sich die Frage, ob eine Vernehmung des Kindes zulässig ist, wenn die Eltern sich trotz des Einigungsgebotes nach $\S 1627$ Satz 2 BGB $^{63}$ nicht auf Zustimmung oder Verweigerung verständigen können.

Nachdem es keine Entscheidungsprärogative zugunsten eines Elternteiles gibt $^{64}$, käme entweder eine Entscheidung des Vormundschaftsgerichts oder die Durchsetzung des sich weigernden Elternteiles in Betracht ${ }^{65}$.

Das Gesetz sieht bei Meinungsverschiedenheiten der Eltern gemäß $\S 1628$ BGB eine Mitwirkung des Vormundschaftsgerichtes nur dann vor, wenn es sich um eine Angelegenheit von erheblicher Bedeutung für das Kind handelt. Der Begriff der erheblichen Bedeutung ist unbestimmt ${ }^{66}$; gleichwohl kann die Entscheidung über die Ausübung eines Zeugnisverweigerungsrechtes diese Voraussetzung erfüllen ${ }^{67}$. Dies wäre beispielsweise denkbar, wenn mittelbar erhebliche Vermögensinteressen des Kindes von dem Ausgang des Rechtstreites berührt werden ${ }^{68}$. Das Vormundschaftsge-

\footnotetext{
${ }^{62}$ Zum Problem der Ausübung nur durch einen Elternteil vgl. auch unten § 6 C IV 2.

${ }^{63}$ Diese Vorschrift bezeichnet Gernhuber, $\S 50$ II 2 als eine der trivialsten Aussagen des BGB.

${ }^{64} \mathrm{Nach} \S 1628$ I BGB a.F. konnte der Vater bei Meinungsverschiedenheiten allein entscheiden; er hatte auf die Auffassung der Mutter lediglich Rücksicht zu nehmen.

${ }^{65}$ Die zweite Alternative wird vertreten von Bosch, S. 52; Grundmann, S. 96; Stein-JonasSchumann, § $383 \mathrm{Rn} 9$.

${ }^{66}$ Gernhuber, $\$ 50$ II 4 unter Hinweis auf die praktischen Schwierigkeiten einer Regelung, die Eingriffe in das Sorgerecht in Ausnahmefällen zuläßt.

${ }^{67}$ Gernhuber, §49. VI 7; a.A. Grundmann, S. 96 ohne zwingende Begründung. Das Bestehen eines Zeugnisverweigerungsrechtes, auf das Grundmann abstellt, ist notwendige Voraussetzung für die Beteiligung der gesetzlichen Vertreter an der Entscheidung und sagt über die tatsächliche Bedeutung des Gewissenskonfliktes für das Kind nichts aus.

${ }^{68}$ Soweit ersichtlich wurde die Ausübung eines Zeugnisverweigerungsrechtes bisher nicht unter dem Gesichtspunkt betrachtet, ob bei Meinungsverschiedenheiten der Eltern das Vormundschaftsgericht angerufen werden kann. Anerkannt ist dies aber bei Differenzen der
} 
richt darf jedoch selbst bei Annahme einer Angelegenheit von erheblicher Bedeutung nur auf Antrag eines Elternteiles im Rahmen des § 1628 BGB tätig werden ${ }^{6}$. Das Tätigwerden des Vormundschaftsgerichtes wäre daher in der Hand der antragsberechtigten Eltern. Eine Verpflichtung zur Anrufung des Vormundschaftsgerichtes besteht nicht. Der Rechtsstreit, in dem das Kind als Zeuge aussagen soll, würde dadurch mit einer erheblichen Unsicherheit belastet, solange unklar ist, ob die Eltern sich noch einigen oder ein Antrag im Sinne des $\S 1628$ BGB gestellt wird. Damit besteht zugleich die Gefahr, daß das Verfahren verzögert wird, weshalb § 1628 BGB als Lösung unbefriedigend bleibt ${ }^{70}$.

Der Notwendigkeit der schnellen Klärung der Entscheidung der Eltern und damit der Zulässigkeit der Vernehmung des minderjährigen Zeugen wird Rechnung getragen, wenn die Weigerung eines Elternteiles ausreicht. Der Schutz des Minderjährigen ist gewahrt, wenn die Vernehmung und Aussage im Zweifel unterbleibt. Im Ergebnis setzt sich somit der zustimmungsverweigernde Elternteil durch. Zu diesem Ergebnis gelangt im übrigen auch, wer die Ausübung des Zeugnisverweigerungsrechts nicht als erhebliche Angelegenheit im Sinne des § 1628 BGB einordnet, denn in solchen Angelegenheiten minderer Bedeutung setzt sich stets der weigernde Elternteil durch ${ }^{71}$. Ferner spricht die Kontrollfunktion der Beteiligung der gesetzlichen Vertreter für die Annahme eines Vetorechtes jedes Ehegat$\operatorname{ten}^{72}$. Als praktische Erwägung ist zu berücksichtigen, daß die Frage der Zulässigkeit der Vernehmung nicht noch zusätzlich mit der im Einzelfall schwierigen Abgrenzungsfrage, ob eine erhebliche Angelegenheit vorliegt, belastet wird.

Eltern über die Anlage größeren Kindesvermögens, so MünchKomm-Hinz, §1628 Rn 11; Soergel-Strätz, § $1628 \mathrm{Rn} 9$.

${ }^{69}$ Erman-Michalski, $\quad \S 1628 \mathrm{Rn} 4 ; \quad$ MünchKomm-Hinz, $\$ 1628 \mathrm{Rn} 4 ; \quad$ Soergel-Strätz, $\S 1628 \mathrm{Rn} 8$.

${ }^{70}$ Auch Bosch, S. 52 hält die Belastung und die Verzögerung des Rechtsstreites für beachtlich. Zustimmend Orlowsky, S. 150 f.

${ }^{71}$ Gernhuber, § 50 II 4; MünchKomm-Hinz, § 1628 Rn 12.

${ }^{72}$ So Orlowsky, S. 152 f. 
b) Vormundschaftsgerichtliche Maßnahmen nach § 1666 BGB?

Ein amtswegiges Einschreiten des Vormundschaftsgerichts kommt allenfalls nach Maßgabe des § 1666 I, II BGB in Betracht. Die bloße Meinungsverschiedenheit der Eltern genügt nicht, es muß eine pflichtwidrige, nicht notwendig schuldhafte Gefährdung des Kindeswohles hinzutreten ${ }^{73}$. Geht man davon aus, daß sich im Zweifel die Zustimmungsverweigerung durchsetzt, stellt sich die Frage, ob gerade das Unterbleiben der Vernehmung trotz Aussagebereitschaft des Kindes einschließlich der Verwertung der Aussage im Urteil die Eingriffsvoraussetzungen des § 1666 BGB erfüllen kann. Angesichts der hohen Anforderungen, die insbesondere an die Gefährdung des Kindeswohles gestellt werden ${ }^{74}$, ist dies nur in seltenen Fällen denkbar. Dennoch ist dies im Einzelfall stets zu prüfen ${ }^{75}$ und gegebenfalls ein Ergänzungspfleger zu bestellen, der an Stelle der sich nicht einigenden Eltern entscheidet.

Die Ersetzung der Zustimmung der gesetzlichen Vertreter durch die vormundschaftsgerichtliche Entscheidung ohne Bestellung eines Pflegers ist gemäß § 1666 II BGB möglich. In Anbetracht der Tatsache, daß im Laufe der Vernehmung weitere Entscheidungen eines Sorgeberechtigten notwendig werden können, etwa im Zusammenhang mit einem Zwischenstreit nach $\S 387$ III ZPO erscheint dies jedoch nicht zweckmäßig. Entweder ist dann nachträglich ein Pfleger zu bestellen oder das Vormundschaftsgericht muß erneut entscheiden. Auf jeden Fall ist die mögliche Beschleunigung des Verfahrens zweifelhaft und gering.

\footnotetext{
${ }^{73}$ Gernhuber, $\$ 49$ VIII $3,4$.

${ }^{74}$ Vgl. etwa Erman-Michalski, § 1666 Rn 8 ff; MünchKomm-Hinz, § 1666 Rn 29 ff; SoergelSträtz, § 1666 Rn 32 wonach eine erhebliche Schädigung des körperlichen, geistigen oder seelischen Wohls mit ziemlicher Sicherheit zu erwarten sein muß. Einschränkend bei der Frage der Zustimmungsbefugnis zur Aussage des Minderjährigen auch Roestel, NJW 1967, 967 f.

${ }^{75}$ RGRK-Wenz, Vor § 1626 Rn 36; ebenda, § 1666 Rn 6.
} 


\section{Beteiligung beider Elternteile am Verfahren}

\section{Ausschlu $\beta$ der gesetzlichen Vertreter}

a) Darstellung des Streitstandes

Sind beide Elternteile am Verfahren als Partei beteiligt, sei es, da $ß$ sie gemeinsam klagen oder verklagt werden, sei es, daß sie sich beispielsweise im Scheidungsverfahren gegenüberstehen, ändert sich zunächst nichts an der festgestellten eigenen Entscheidungsbefugnis des minderjährigen Zeugen. Ebenso unzweifelhaft ist wegen der sich aufdrängenden Gefahr einer Interessenkollision, daß die gesetzlichen Vertreter infolge ihrer Verfahrensbeteiligung bei der Zustimmung zur Aussage des Kindes ausgeschlossen sind ${ }^{76}$. Die Einzelheiten sind jedoch nach wie vor ungeklärt.

Im wesentlichen werden hierzu zwei Auffassungen vertreten, die in ihren unterschiedlichen Auswirkungen stark voneinander abweichen. Nach einer vorwiegend von der Rechtsprechung vertretenen Meinung kann den Eltern durch das Vormundschaftsgericht die gesetzliche Vertretungsmacht durch die Bestellung eines Ergänzungspflegers analog den § 1629 II 3, § 1796 BGB entzogen werden ${ }^{7}$. Die Gegenmeinung nimmt dagegen keine Entziehung der Vertretungsmacht, sondern in analoger Anwendung der § 1629 II 1 , $\S 1795$ I Nr. 3 BGB den Ausschluß der Eltern kraft Gesetzes an ${ }^{78}$. Nach der

\footnotetext{
${ }^{76}$ Gernhuber, § 51 IV 1; Soergel-Strätz, § 1629 Rn 18; Stein-Jonas-Schumann, § 383 Rn 10. Das OLG Frankfurt, MDR 1987, 151, 152 hält die Frage des Ausschlusses für unerheblich, weil der Pfleger keinen Entscheidungsspielraum habe und im Interesse des Kindes stets das Zeugnisverweigerungsrecht ausüben müßte. Eine Verallgemeinerung dieser Feststellung liefe darauf hinaus, bei kindlichen Zeugen bei Vorliegen eines Zeugnisverweigerungsrechts auf den Zeugen zu verzichten. Dies entspricht im Ergebnis der Auffassung von K. Peters; dazu oben § 5 A II 2 a.

${ }^{7}$ BayObLG, NJW 1967, 206, 209 = FamRZ 1966, 644, 647; OLG Stuttgart, NJW 1971, 2237, 2239 f; OLG Hamm, OLGZ 1972, 157, 158; LG Mannheim, FamRZ 1974, 158; LG Frankfurt, FamRZ 1974, 378, 379; Bosch, S. 54; Stein-Jonas-Schumann, § 383 Rn 10; Zöller-Stephan, § 383 $\mathrm{Rn} 4$;

${ }^{78}$ OLG Stuttgart, FamRZ 1965, 515, 517; wohl auch OLG Zweibrücken, FamRZ 1980, 911; Gernhuber, § 51 IV 1 Fn 3; Grundmann, S. 94 f zum Scheidungsrechtsstreit; Hampel, FamRZ 1964, 125, 128; MünchKomm-Hinz, § 1626 Rn 50; Schaub, FamRZ 1966, 134, 136 zur StPO vor 1974; Schneider, JurBüro 1970, 1021, 1022; Soergel-Damrau, § 1795 Rn 8; ebenda, § 1629 Rn 18; Staudinger-Donau, § 1626 Rn 75. Unklar Roestel, NJW 1967, 967 f.
} 
erstgenannten Auffassung entfällt die Zustimmungsbefugnis daher erst mit der konstitutiv wirkenden Entscheidung des Vormundschaftsgerichtes; der Beschluß des Vormundschaftsgerichtes wird gemäß § 16 I FGG mit der Bekanntmachung gegenüber den Eltern wirksam. Die Unterschiede beider Auffassungen werden deutlich, wenn man bedenkt, daß die Vernehmung des aussagebereiten Minderjährigen nur nach der vorher erklärten eindeutigen Zustimmung der gesetzlichen Vertreter zulässig ist ${ }^{79}$. Geht man daher von der Verhinderung der gesetzlichen Vertreter von Anfang an aus, so bedeutet dies, daß vor der Vernehmung des Minderjährigen immer die Bestellung eines Ergänzungspflegers gem. § 1909 I BGB und damit die Durchführung des vormundschaftsgerichtlichen Verfahrens abgewartet werden müsste.

b) Stellungnahme

Die insbesondere vom $O L G$ Stuttgart ${ }^{80}$ zum Strafprozeß vertretene Anwendung der § 1629 II 3, § 1796 BGB nimmt für sich in Anspruch, eine Verzögerung des Verfahrens durch die Einschaltung des Vormundschaftsgerichtes zu vermeiden. Darüberhinaus besteht nach dieser Ansicht nur dann ein konkreter Interessenskonflikt zwischen den gesetzlichen Vertretern und dem aussagebereiten Kind, wenn die Zustimmung verweigert wird ${ }^{81}$. Eine abstrakte Gefährdung der Kindesinteressen, wie sie nach § 1795 I Nr. 3 BGB vorliegen müßte ${ }^{82}$, wird damit für den vorliegenden Fall abgelehnt. Als weiteres Argument ließe sich anführen, daß ein gesetzlicher Ausschluß der Vertretungsmacht nur in den in § 1795 I BGB enumerierten Fällen vorgese-

\footnotetext{
${ }^{79}$ OLG Stuttgart, OLGZ 1985, 385, 388 = FamRZ 1985, 1154, 1155; oben § 6 C II 1 c. Daran ändert auch die Möglichkeit nachträglicher Genehmigung durch die gesetzlichen Vertreter nichts, weil diese nur dann wirksam werden kann, wenn die Aussagebereitschaft fortbesteht und am Inhalt der Aussage festgehalten wird.

${ }^{80} \mathrm{AaO}$ (Fn 77).

${ }^{81}$ Ebenda. Das Argument des LG Frankfurt, FamRZ 1974, 378, 379, der Ausschluß der Eltern kraft Gesetzes sei mit der Unschuldsvermutung des Strafverfahrens unvereinbar, kann auf den Zivilprozeß nicht übertragen werden.

${ }^{82}$ Bosch, S. 53 stellt darauf ab, daß $\S 1795$ I Nr. 3 BGB einen Rechtsstreit zwischen Sorgeberechtigten und Kind voraussetze und dies erst bei einem Zwischenstreit gemäß § 387 ZPO gegeben sein könne. Eine analoge Anwendung zieht Bosch nicht in Betracht. MünchKommSchwab, § 1795 Rn 1; Soergel-Damrau, § 1795 Rn 1; Schwab, Rn 452.
} 
hen ist; der Anwendungsbereich der Vorschrift soll aus Gründen der Rechtssicherheit nicht in extensiver Analogie erweitert werden ${ }^{83}$.

Im Schriftum wird dieser Meinung zu Recht entgegen gehalten, daß schon die prozessuale Beteiligung der Eltern unter dem Gesichtspunkt der Interessenkollision und dem Rechtsgedanken des §181 BGB ihren Auschluß ex lege und von Anfang an geboten sein läßt ${ }^{84}$. In diesen Fällen ist von einer dem Regelungszweck des § 1795 BGB entsprechenden abstrakten Gefährdung der Kindesinteressen auszugehen, da nicht, wie vom $O L G$ Stuttgart angenommen, nur die Zustimmungsverweigerung eine Interessenkollision offenbart, sondern bei jeder Entscheidung der Eltern denkbar ist, die sich nicht am objektiv zu bestimmenden Kindeswohl orientiert. Die Zustimmungsbefugnis dient gerade dem Schutz des aussagebereiten Minderjährigen, da er die Folgen seiner Aussage für sich selbst oder seine Angehörigen nicht überblicken und daher keine verantwortliche Entscheidung über sein Zeugnisverweigerungsrecht treffen kann. Es kommt daher nicht auf den formalen Gegensatz zwischen Zustimmungsverweigerung und Aussagebereitschaft an, sondern auf die Abwägung zwischen den Folgen einer Aussage und einer Aussageverweigerung. Danach kann aber die Zustimmung der Eltern den objektiv verstandenen Interessen des Kindes ebenso widerstreiten wie eine Zustimmungsverweigerung.

Auch die Gefahr der Verzögerung des Zivilprozesses durch die regelmäBig erforderliche vormundschaftsgerichtliche Entscheidung kann keine andere Beurteilung rechtfertigen. Die Verzögerung ist eine unmittelbare und notwendige Folge des Minderjährigenschutzes und daher von den Beteiligten hinzunehmen ${ }^{85}$. Im übrigen kann auch die gegenteilige Ansicht die nachträgliche Anrufung des Vormundschaftsgerichts nicht in jedem Fall vermeiden. Stellt sich nach Abschluß der Vernehmung ein erheblicher Interessenkonflikt zwischen kindlichem Zeugen und zustimmenden Eltern

\footnotetext{
${ }^{83}$ MünchKomm-Schwab, ebenda (Fn 82).

${ }^{84}$ Gernhuber, § 49 VI 7; § 51 IV 1. Für die Anwendung der § 1629 II 1, § 1795 I Nr. 3 BGB im Strafverfahren vor der Einführung des $\S 52$ II StPO Orlowsky, S. 166 ff. Schimansky, FS f. Pfeiffer, S. 297, 299 sieht in der Neufassung des § 52 II StPO eine Entscheidung des Gesetzgebers zugunsten dieser Ansicht - im Ergebnis zu Recht.

${ }^{85}$ So auch Bosch, S. 54. Nach BVerfG, FamRZ 1986, 871, 874 = NJW 1986, 3129, 3130 ist bei einer Kollision des Eltern- und des Kindesinteresses stets den Interessen des Kindes der Vorrang einzuräumen. Der nachgeordnete Rang der Eltern kommt m.E. auch darin zum Ausdruck, da B sie im Interesse des Kindes Einschränkungen in ihrem Recht auf Beweis hinnehmen müssen. Im Verhältnis zu Dritten gilt dies ebenfalls.
} 
heraus, müßte auch nach der Gegenansicht ein Ergänzungspfleger bestellt werden. Erst nach dessen Zustimmung könnte die Aussage bei noch fortdauernder Aussagebereitschaft des Zeugen verwertet werden. Dem kann nicht entgegengehalten werden, daß es nach der Vollendung der Aussage am Fürsorgebedürfnis und damit an einer Voraussetzung für die partielle Entziehung der elterlichen Sorge fehle, weil der Interessenkonflikt sonst regelmäßig ohne Auswirkungen bliebe. Das Argument der Verfahrensbeschleunigung greift daher nicht; es rechtfertigt insbesondere keine Verkürzung des notwendigen Schutzes des minderjährigen Zeugen.

Für den gesetzlichen Ausschluß der Eltern spricht auch die Übereinstimmung mit der strafprozessualen Regelung in $\$ 52$ II 2 StPO, die ebenfalls den automatischen Ausschluß der gesetzlichen Vertreter anordnet ${ }^{86}$. Eine entsprechende Handhabung im Zivilprozeß ist sachlich gerechtfertigt und vermeidet im übrigen Differenzen zwischen Adhäsionsverfahren und normalem Zivilproze $\beta^{87}$.

Desweiteren führt die entsprechende Anwendung des § 1796 BGB nicht zu größtmöglichem Schutz des Minderjährigen, weil die Gefahr nicht vermieden wird, daß der Minderjährige zunächst, gestützt auf die Zustimmung der Eltern, aussagt, die Aussage im nachhinein aber durch die Zustimmungsverweigerung des später bestellten Pflegers unverwertbar wird. Die Belastung des Kindes durch die Vernehmungssituation läßt sich nicht rückgängig machen.

Zusammenfassend ist festzustellen, daß die gesetzlichen Vertreter im Fall der eigenen Beteiligung an dem Verfahren von der Zustimmungsbefugnis entsprechend den $\S 1629$ II 1, 1795 I Nr. 3 BGB ausgeschlossen sind und immer ein Ergänzungspfleger zu bestellen ist.

Eine Genehmigung der Zustimmung der ausgeschlossenen Eltern durch den Pfleger ist nicht ohne weiteres möglich; die §§ 184, 185 BGB sind im Prozeßrecht nicht entsprechend heranzuziehen ${ }^{88}$. Wie bei der nachträglichen Zustimmung durch die nicht beteiligten gesetzlichen Vertreter muß auch hier der Wille des Kindes hinzutreten, an der einmal gemachten Aussage festhalten zu wollen ${ }^{89}$.

\footnotetext{
${ }^{86}$ Löwe-Rosenberg-Dahs, § $52 \mathrm{Rn} 32$.

${ }^{87}$ Vgl. unten § 6 C IV.

${ }^{88}$ Soergel-Leptien, Vor § $182 \mathrm{Rn} 17$.

${ }^{89} \mathrm{Vgl}$. oben $\S 6 \mathrm{C}$ II $1 \mathrm{c}$.
} 


\section{Bestellung eines Pflegers durch das Vormundschaftsgericht}

Das Verfahren vor dem Vormundschaftsgericht wird im dritten Abschnitt dieser Untersuchung dargestellt. Soweit im folgenden von der Entscheidung des Ergänzungspflegers die Rede ist, wird davon ausgegangen, daß der Pfleger wirksam bestellt wurde. Seine Entscheidung tritt an die der Eltern, die diese als gesetzliche Vertreter sonst in der entsprechenden Situation treffen könnten.

\section{Beteiligung eines Elternteils am Verfahren}

Die letzte zu behandelnde Fallgruppe betrifft die Beteiligung nur eines Elternteiles am Verfahren. Im Regelfall üben beide Eltern das Sorgerecht gemeinsam aus, §1626 I 1, §§ 1627, § 1629 I 2 BGB. Dennoch sind Fälle denkbar, in denen das Sorgerecht bereits vor dem Zivilverfahren, in dem ein Elternteil beteiligt ist, nur einem Ehegatten zustand. Es ist daher zu differenzieren, je nach dem, ob der beteiligte Elternteil zugleich alleiniger Sorgerechtsinhaber ist, ob dem anderen Teil das Sorgerecht ausschließlich zusteht oder ob, wie im genannten Regelfall, beide Eltern gemeinsam sorgeberechtigt sind.

\section{Gemeinsame Sorgerechtsausübung beider Eltern}

Auch hier gilt, daß der Grundsatz der Gesamtvertretung wegen der Gefahr der Interessenkollision nicht zum Tragen kommen kann, mithin zumindest der am Prozeß beteiligte Elternteil nicht befugt ist, die erforderliche Zustimmung zur Aussage des minderjährigen Zeugen zu erteilen oder zu versagen. Offen ist allerdings, ob die Zustimmungsbefugnis auf den nicht beteiligten Ehegatten übergeht, ob also dessen Vertretungsmacht analog $\S 1680$ II 2 BGB zur Alleinvertretungsmacht erstarkt, sofern für ihn nicht zusätzliche Ausschlußgründe vorliegen ${ }^{90}$, oder ob auch hier, wie die Gegen-

\footnotetext{
${ }^{90}$ BayObLG, NJW 1967, 206, 209 = FamRZ 1966, 644, 647; OLG Stuttgart, NJW 1971, 2237,
} 2239 f; LG Frankfurt, FamRZ 1974, 378, 379; Baumbach-Lauterbach-Hartmann, Einf. §§ 383- 
ansicht annimmt, analog § 52 II 2 StPO ein Ausschluß beider Elternteile anzunehmen ist ${ }^{11}$.

Die Annahme eines Erstarkens der Gesamt- zur Alleinvertretungsmacht oder besser Alleinzuständigkeit des nicht Beteiligten bedeutet eine Abweichung von der Rechtslage im Strafprozeß, in dem immer beide Eltern ausgeschlossen sind. Eine entsprechende Regelung im Zivilprozeß ist zwar nicht allein schon mit dem Argument zu begründen, der Lückenhaftigkeit der Zivilprozeßordnung sei anders nicht zu begegnen, zumal § 52 II 2 StPO in seinen Auswirkungen noch nicht als abschließend geklärt angesehen werden kann ${ }^{92}$. Für die Zweckmäßigkeit einer gleichartigen Handhabung spricht aber neben familienrechtlichen Erwägungen auch die Vermeidung eines kaum lösbaren Widerspruchs zur Rechtsfolge im Adhäsionsverfahren. In diesem Verfahren können nach $\S \$ 403 \mathrm{ff}$ StPO vermögensrechtliche Ansprüche, die auf einer Straftat beruhen, im Strafverfahren geltend gemacht und entschieden werden. Die Entscheidung über den Schadensersatzanspruch folgt strafprozessualen Regelungen, insbesondere soweit es die Beweisaufnahme betrifft ${ }^{93}$. Dementsprechend käme $\S 52$ II 2 StPO uneingeschränkt zur Anwendung. Würde derselbe Anspruch in einem gesondertem Zivilverfahren geltend gemacht, so könnte nach der dargestellten Meinung der nicht beteiligte Ehegatte der Vernehmung eines kindlichen Zeugen zustimmen, im Adhäsionsverfahren müßte zuerst ein Pfleger bestellt werden. Eine sachliche Rechtfertigung für diese unterschiedliche Zustimmungsbefugnis ist unbeschadet der geringen praktischen Bedeutung des Adhäsionsverfahrens ${ }^{94}$ nicht ersichtlich.

Nach der hier vertretenen Auffassung sind bei Beteiligung beider Eltern die gesetzlichen Vertreter von Anfang an und kraft Gesetzes von der Vertretung ausgeschlossen ${ }^{95}$, weil wegen der Beteiligung eine abstrakte Gefährdung der vorrangig schutzwürdigen Kindesinteressen gegeben ist. Die

389 Anm. 3; Stein-Jonas-Schumann, §383 Rn 10; Wieczorek, §383 Anm. A IV a; ZöllerStephan, § $383 \mathrm{Rn} 4$; ebenso wohl auch Grundmann, S. 95 f; MünchKomm-Hinz, § $1626 \mathrm{Rn}$ 48.

${ }^{91}$ Bosch, S. 55; Ern:an-Holzhauer, § 1909 Rn 4; Gernhuber, §49 VI 7; Schoene, NJW 1972, 930; Soergel-Strätz, § 1629 Rn 18. Unklar Erman-Michalski, § $1626 \mathrm{Rn} 20$.

${ }^{92} \mathrm{Vgl}$. dazu auch unten $\S 6 \mathrm{C}$ IV 2 zur Frage der teleologischen Reduktion der Vorschrift.

${ }^{93}$ Löwe-Rosenberg-Wendisch, § $404 \mathrm{Rn} 8$.

${ }^{94}$ Ebenda, Vor $\S 403 \mathrm{Rn} 8 \mathrm{f}$.

${ }^{95}$ Oben, § 6 C III 1. 
§ 1629 II 1, § 1795 I BGB sind entsprechend anwendbar. Gerade für den Anwendungsbereich dieser Vorschriften entspricht es aber allgemeiner Überzeugung, daß die Interessen des Kindes auch dann gefährdet sind, wenn die Vertretungsmacht auf den nicht beteiligten Ehegatten übergeht, besonders wenn zwischen den Eltern gutes Einvernehmen herrscht. In diesem Fall soll daher auch der Unbeteiligte von der gesetzlichen Vertretung ausgeschlossen $\operatorname{sein}^{96}$. Begründet wird dies mit dem Wortlaut des § 1629 II 1 BGB, der beide Elternteile nennt und einem Umkehrschluß aus $\S 1678$ BGB, der in den Fällen des § 1795 BGB keine Sorgerechtsausübung durch den nicht ausgeschlossenen Ehegatten vorsieht ${ }^{97}$.

Für die Ausübung des Zeugnisverweigerungsrechtes durch den Minderjährigen bedeutet dies, da $\beta$ auch bei Beteiligung nur eines Elternteiles ein Ergänzungspfleger nach § 1909 BGB bestellt werden muß. Dessen Entscheidung ersetzt die der ausgeschlossenen gesetzlichen Vertreter. Das Verfahren entspricht dem bei Beteiligung beider Elternteile, so daß auf die dortigen Ausführungen verwiesen werden kann ${ }^{98}$.

\section{Sorgerechtsausübung durch einen Elternteil}

Die oben erwähnte Frage nach dem Erstarken der Gesamtvertretung zur Alleinvertretung bzw. alleinigen Zuständigkeit des nicht beteiligten Ehegatten kann sich naturgemäß nur stellen, wenn beide Eltern das Sorgerecht gemeinsam ausüben. Soweit dies nicht der Fall ist ${ }^{99}$, bestehen keine Besonderheiten. Es muß aber unterschieden werden, ob der Sorgerechtsinhaber oder der ausgeschlossene Elternteil am Verfahren beteiligt ist.

\footnotetext{
${ }^{96}$ BayObLG, NJW 1976, 168; BGH, NJW 1972, 1708; OLG Zweibrücken, FamRZ 1980, 911 f; MünchKomm-Hinz, § 1629 Rn 18; RGRK-Wenz, § 1626 Rn 24; Schwab, Rn 453; Soergel-Strätz, $\S 1629 \mathrm{Rn} 25$. Aus diesem Grund kann auch dem Vorschlag von Bosch, S. 55, der einen TeilAusschluß nur des beteiligten Ehegatten in Erwägung zieht und den unbeteiligten Elternteil zusammen mit dem Ergänzungspfleger entscheiden lassen will, nicht gefolgt werden. Wie hier im Strafverfahren bei Anklage gegen einen Elternteil Schaub, FamRZ 1966, 134, 135 f; Roestel, RdJB 1971, 218, 219 f.

${ }^{97}$ Soergel-Strätz, § $1629 \mathrm{Rn} 25$.

${ }^{98}$ Oben $\S 6$ C III.

${ }^{99}$ Etwa weil ein Ehegatte aus rechtlichen oder tatsächlichen Gründen, die von dem anhängigen Zivilverfahren, in dem das Kind als Zeuge aussagen soll, unabhängig sind, verhindert ist und das Sorgerecht daher gem. §§ $1673 \mathrm{ff}$ BGB ruht; allgemein dazu Gernhuber, § 55.
} 
Der alleinige Sorgerechtsinhaber, der zugleich Partei des Prozesses ist, kann die Zustimmung oder die Ablehnung zu der Aussage des Kindes als Zeuge nicht erklären. Der Interessenkonflikt und die daraus folgende Gefährdung des Kindeswohles ist in gleicher Weise gegeben, als wären beide sorgerechtsberechtigte Eltern am Prozeß beteiligt ${ }^{100}$.

Im umgekehrten Falle fehlt es an einer vergleichbaren Konfliktsituation, weil Zustimmungsbefugnis und Prozeßbeteiligung nicht in einer Person zusammentreffen. In Übereinstimmung mit der Rechtslage im Strafverfahren ist der nichtbeteiligte Elternteil zur Entscheidung über die Aussage des Kindes befugt.

Schimansky vertritt zu §52 II 2 StPO die Meinung, die Einschränkung des Ausschlusses beider Ehegatten auf die Fallgestaltung, daß beiden Eltern das Sorgerecht gemeinsam zusteht, beruhe auf einem Redaktionsversehen und sei eine gesetzgeberische Fehlleistung ${ }^{101}$. Die Gefahr der Interessenkollision beruhe nicht auf der Verteilung des Sorgerechts, sondern auf der ehelichen Bindung der Ehegatten. Deshalb habe der Strafgesetzgeber einen falschen Ansatzpunkt gewählt, als er in §52 II 2 StPO an die gesetzliche Vertretung angeknüpft habe ${ }^{102}$. Nach dieser Auffassung ist $\S 52$ II StPO im Wege der teleologischen Reduktion so auszulegen, daß, entsprechend dem Grundgedanken des $\S 1629$ II 1, § 1795 I Nr. 3 BGB, unabhängig von der Verteilung des Sorgerecht immer beide Eltern von der Zustimmungsbefugnis ausgeschlossen sind ${ }^{103}$.

Die vorgestellte Argumentation ist auf das Zivilverfahren an sich ohne weiteres übertragbar. Gleichwohl kann ihr nicht gefolgt werden. Der Kritik an $\S 52$ II 2 StPO ist zuzugeben, daß die Gefährdung des Kindeswohls nicht von der Frage abhängt, ob Gesamt- oder Alleinvertretung vorliegt, sondern auf der engen familiären Beziehung der Eltern untereinander und gegen-

\footnotetext{
${ }^{100} \mathrm{Vgl}$. dazu oben $\S 6$ C III.

${ }^{101}$ Schimansky, FS f. Pfeiffer, S. 297, 298 ff; KMR-Paulus, § 52 Rn 25.

${ }^{102}$ Schimansky, ebenda, S. $297,300 \mathrm{ff}$.

${ }^{103}$ Auf die weitergehende Streitfrage, ob ein Ausschluß der Eltern auch anzunehmen sei, wenn Beschuldigter nicht der Ehegatte, sondern ein naher Verwandter des alleinigen Sorgerechtsinhabers ist, braucht im vorliegenden Zusammenhang nicht näher eingegangen werden, weil nach hier vertretener Ansicht schon bei Beteiligung eines Elternteiles ohne gemeinsames Sorgerecht kein automatischer Ausschluß angenommen werden kann. Zur Rechtslage bei gemeinsamer Sorgerechtsausübung oben § 6 C IV 1.
} 
über dem Kind ${ }^{104}$. Dennoch ist eine teleologische Reduktion contra legem und gegen den eindeutigen Wortlaut weder im Strafverfahren noch im Zivilverfahren erforderlich ${ }^{105}$.

Der Ausschluß des beteiligten Ehegatten von der Zustimmungsbefugnis beruht auf der abstrakten Gefährdung des Kindeswohls durch die stets gegebene Interessenkollision. Diese abstrakte Gefährdung kann nicht in gleichem Maße angenommen werden, wenn von vornherein nur der Elternteil zur Zustimmung bzw. Verweigerung derselben befugt ist, der am Verfahren nicht unmittelbar beteiligt ist. Auch kann gerade die Tatsache, daß nur ein Elternteil sorgeberechtigt ist, dazu führen, daß keine Interessenkollision wahrscheinlich ist. So kann bei einem Rechtsstreit des geschiedenen oder getrenntlebenden Ehegatten ${ }^{106}$ die familiäre Beziehung bereits mehr oder weniger gelockert sein und somit eine abstrakte Gefährdung der Kindesinteressen durch die Entscheidungsbefugnis des sorgeberechtigten Elternteiles nicht unterstellt werden. Durch eine teleologische Reduktion des $\S 52$ II 2 StPO und die entsprechende Anwendung der so verstandenen Vorschrift im Zivilprozeß würde der Grund der besonderen Sorgerechtsregelung unberücksichtigt bleiben, obwohl sie notwendig Auswirkungen auf die familiären Beziehungen hat, die nach der Ansicht Schimanskys gerade entscheidend sind.

Das schließt im Einzelfall eine Interessenkollision nicht aus; der Schutz des Minderjährigen verlangt aber m.E. hier erst bei Vorliegen konkreter Anhaltspunkte ein Eingreifen des Vormundschaftsgerichtes ${ }^{107}$. Im Ergebnis

${ }^{104}$ Ähnlich auch Roestel, RdJB 1971, 218, 220, der eine Art "Gesamthaftung" der Eltern annimmt.

${ }^{105}$ Auch die wohl überwiegende Ansicht zum Strafprozeß lehnt eine Reduktion des §5 II 2 StPO ab; Kleinknecht/Meyer, § 52 Rn 20; Löwe-Rosenberg-Dahs, § 52 Rn 32 jeweils m.w.N.; Rieß, NJW 1975, 81, 83 Fn 42 erwägt eine Reduktion allenfalls bei anderen Verwandten als Beschuldigte, hält den Gesetzestext ansonsten aber für eindeutig. Offen gelassen in $B G H$ NStZ 1988, 17 f.

${ }^{106}$ Nach $\S \S 1671,1672$ BGB kann das Sorgerecht auf einen Elternteil übertragen werden, wenn dies zum Wohl des Kindes erforderlich ist; daran hat die Entscheidung BVerfGE 61, $358 \mathrm{ff}=$ FamRZ 1982, 1179, durch die § 1671 IV 1 BGB für verfassungswidrig erklärt wurde, nichts geändert; dazu MünchKomm-Hinz, § $1671 \mathrm{Rn} 4$; Soergel-Strätz, § $1671 \mathrm{Rn} 1,18$ f. Das Zeugnisverweigerungsrecht des Kindes nach $\S 383$ I Nr. 3 ZPO wird durch die Scheidung der Eltern nicht berührt, da das Verwandschaftsverhältnis fortbesteht.

${ }^{107}$ Auch Schimansky, FS f. Pfeiffer, S. 297, 302, räumt ein, daß der Schutz des Minderjährigen durch die Möglichkeit der Entziehung des Sorgerechts hinreichend gewahrt ist, wenn - im Strafverfahren - Beschuldigter nicht der Ehegatte, sondern ein naher Verwandter ist. Den 
bedeutet dies, daß der Sorgerechtsinhaber zur Entscheidung über die Zeugenaussage des Kindes zuständig bleibt, bis daß Vormundschaftsgericht analog den § 1629 II 3, § 1796 BGB das Sorgerecht insoweit auf einen Ergänzungspfleger überträgt.

\section{Ausübung des Zeugnisverweigerungsrechts}

\section{Die Erklärung der Zeugnisverweigerung durch einen einsichtsfähigen Minderjährigen}

Jedes Zeugnisverweigerungsrecht muß gemäß §386 I ZPO entweder schriftlich oder zu Protokoll der Geschäftsstelle oder mündlich im Vernehmungstermin durch Erklärung geltend gemacht werden ${ }^{108}$. Eine Beachtung des Zeugnisverweigerungsrechtes von Amts wegen kommt nicht in Betracht, da es in der Entscheidung des Zeugen liegt, ob er die prozessuale Einrede geltend macht oder aussagt.

Die Erklärung über die Zeugnisverweigerung muß auch den oder die Weigerungsgründe enthalten, die glaubhaft zu machen sind; insoweit ist der Zeuge im Rahmen der Pflicht zur wahrheitsgemäßen Aussage wenigstens verpflichtet, die Umstände, aus denen er sein Weigerungsrecht ableitet, zu offenbaren ${ }^{109}$.

\footnotetext{
Grund sieht er darin, daß die Entscheidung über das Zeugnisverweigerungsrecht in einem staatlich geordnetem Verfahren und meist in Anwesenheit eines Richters erfolge. Da dies aber immer der Fall ist, weil die Zeugnisverweigerung stets gegenüber dem Gericht erklärt werden muß, kann dieses Argument die differenzierende Haltung Schimanskys nicht begründen.

${ }^{108}$ Kommen mehrere Zeugnisverweigerungsrechte in Betracht, so genügt die Angabe eines Grundes. Hinsichtlich des (noch) nicht geltend gemachten Umstandes tritt keine Verwirkung oder Präklusion ein, Stein-Jonas-Schumann, §386 Rn 6 m.w.N.. Dies ist insbesondere von Bedeutung, wenn der minderjährige Zeuge die erforderliche Einsichtsfähigkeit nur bezüglich eines Zeugnisverweigerungsrechtes besitzt. Die gesetzlichen Vertreter sind dann nach der erfolglosen Geltendmachung der Zeugnisverweigerung durch das Kind nicht an der Erhebung anders begründeter Zeugnisverweigerungsrechte, etwa nach $\$ 384 \mathrm{Nr} .1 \mathrm{ZPO}$, gehindert, indem sie dennoch die Zustimmung verweigern und die Gründe für das Zeugnisverweigerungsrecht glaubhaft machen.

${ }^{109}$ Baumbach-Lauterbach-Hartmann, § 386 Anm. 1; Stein-Jonas-Schumann, § 386 Rn 1 Fn 4.
} 
Beim minderjährigen Zeugen ist auch hier grundsätzlich danach zu unterscheiden, ob die notwendige Verstandesreife vorliegt, sich von dem Zeugnisverweigerungsrecht eine genügende Vorstellung machen zu können. Sind die geistigen Fähigkeiten bereits hinreichend entwickelt, so ist die Minderjährigkeit kein wesentlicher Faktor, da hier eine Gleichstellung zum voll Geschäfts- und Prozeßfähigen gerechtfertigt ist ${ }^{110}$. Die Gleichstellung ergreift auch das Verfahren zur Ausübung des Rechtes, wie es in § 386 ZPO geregelt ist, es sei denn, die Einsichtsfähigkeit läge nur hinsichtlich des Aussageverweigerungsrechtes vor, nicht aber in Bezug auf die vom Zeugen vorzunehmenden Verfahrenshandlungen, die zur Herbeiführung der Befreiung von den Zeugenpflichten notwendig sind. Ein derartiger Fall erscheint jedoch kaum denkbar, da die Entscheidung über die Ausübung des Zeugnisverweigerungsrechtes komplexer Natur ist, während die Art der Geltendmachung im Verfahren demgegenüber keine besonderen geistigen Fähigkeiten erfordert. Darüberhinaus bestehen hier keine Bedenken gegen eine Mitwirkung der gesetzlichen Vertreter, die insoweit keine eigene Entscheidung treffen.

\section{Ausübung durch einen nicht Einsichtsfähigen}

Der nicht einsichtsfähige Minderjährige darf nur aussagen, wenn er hierzu bereit ist und die gesetzlichen Vertreter oder bei deren Verhinderung der Ergänzungspfleger der Aussage zustimmt. Dieser Zeuge kann somit als Beweismittel entweder wegen fehlender Aussagebereitschaft oder wegen fehlender Zustimmung ausfallen.

Fehlt lediglich die Zustimmung zur Aussage des selbst aussagebereiten Zeugen, so spricht nichts dagegen, daß der Minderjährige bei Abgabe der Erklärung im Sinne des §386 I ZPO von dem gesetzlichen Vertreter bzw. dem Pfleger vertreten wird und dieser auch die tatsächlichen Gründe, aus denen das Weigerungsrecht abgeleitet wird, darlegt und glaubhaft macht. Die Geltendmachung ist nicht mit der Entscheidung über die Ausübung des Zeugnisverweigerungsrechtes identisch, insbesondere handelt es sich nicht um eine höchstpersönliche Handlung.

${ }^{110} \mathrm{Vgl}$. oben $\S 6 \mathrm{~B}$. 
Sind die Eltern nicht am Verfahren beteiligt und besteht daher nicht die abstrakte Gefahr einer Interessenkollision, können sie den Minderjährigen auch bei fehlender Aussagebereitschaft bei der Erklärung und der Glaubhaftmachung vertreten; auf ihre Zustimmung oder Ablehnung kommt es dann nicht an.

\section{Ausschluß der nicht am Verfahren beteiligten gesetzlichen Vertreter bei konkreter Interessenkollision}

Etwas anders ist jedoch der Fall zu beurteilen, wenn dem Minderjährigen von Anfang an die Aussagebereitschaft fehlt und die Eltern am Verfahren beteiligt sind. Ein Ergänzungspfleger, dem die Entscheidungsbefugnis über die Zustimmung übertragen wird, darf dann nicht bestellt werden, weil eine Vernehmung gegen den erklärten Willen des weigerungsberechtigten Minderjährigen auch mit Zustimmung des gesetzlichen Vertreters auf keinen Fall in Betracht kommt. Eine Interessenkollision und die mögliche Gefährdung der Kindesinteressen ist dann ausgeschlossen; damit entfällt aber auch die Rechtfertigung für den Ausschluß der Eltern und die teilweise Übertragung des Personensorgerechts auf einen Pfleger ${ }^{111}$. Da wegen der fehlenden Einsichtsfähigkeit eine verantwortliche Ausübung des Zeugnisverweigerungsrechtes ebensowenig wie die Glaubhaftmachung der maßgeblichen Umstände erwartet werden kann, andererseits die auf der Höchstpersönlichkeit einer Gewissensentscheidung beruhenden Bedenken hier nicht Platz greifen, sind die Eltern als gesetzliche Vertreter zur Abgabe der erforderlichen Erklärung berufen, wenn die Aussagebereitschaft von Anfang an fehlt. Die Schutzbedürftigkeit des Kindes ist in diesen Fällen geringer, da der Minderjährige nur vor den Folgen einer Aussage, nicht vor denen der Aussageverweigerung geschützt werden soll.

Eine Gefährdung der Interessen des Kindes durch die zustimmungsbefugten Eltern ist jedoch in der Form denkbar, daß diese - etwa beim Aussageverweigerungsrecht gem. §384 Nr. 1 ZPO - die maßgeblichen Umstän-

\footnotetext{
${ }^{111}$ So im Ergebnis (Pflegerbestellung erst zulässig, wenn Aussagebereitschaft festgestellt) OLG Hamm, OLGZ 1972, 157; LG Mannheim, FamRZ 1974, 158; OLG Stuttgart, OLGZ 1985, 385, $386=$ FamRZ 1985, 1154, 1155. Dazu auch unten $\S 11$ B I.
} 
de nicht hinreichend glaubhaft machen und damit mittelbar zu einem prozeßrechtswidrigen Aussagezwang beitragen könnten. Diese Gefahr ist jedoch mit der Gefährdung des Minderjährigen bei gegebener Aussagebereitschaft nicht vergleichbar; daher genügt es m.E. in einem solchen Fall, den Eltern die Personensorge und damit die Zustimmungsbefugnis zu belassen, bis aufgrund konkreter Umstände die Entziehung der Vertretungsmacht und Übertragung auf einen Ergänzungspfleger analog §§ 1629 II 3, 1796 BGB möglich ist.

Bei Beteiligung nur eines Elternteils steht dann bis zu einer konstitutiv wirkenden Entscheidung des Vormundschaftsgerichts die Entscheidungsbefugnis ebenfalls beiden Eltern zu; das Vormundschaftsgericht kann bei Vorliegen einer Interessenkollission gem. §1680 I BGB die alleinige Vertretungsbefugnis auf den unbeteiligten Ehegatten oder einen Ergänzungspfleger übertragen ${ }^{112}$.

Als Ergebnis bleibt festzuhalten, daß die Eltern bezüglich der Erklärungshandlung im Sinne des $\$ 386$ ZPO den nicht einsichtsfähigen Jugendlichen vertreten, es sei denn, wegen der Beteiligung der Eltern und der anfänglichen oder im Laufe des Verfahrens festgestellten Aussagebereitschaft des Zeugen wurde analog §§ 1629 II 1, 1795 I Nr. 3 BGB ein Ergänzungspfleger bestellt. Bei fehlender Aussagebereitschaft von Anfang an kommt die Entziehung der Vertretungsmacht durch die Bestellung eines Ergänzungspflegers nur bei konkreter Interessensgefährdung nach Maßgabe der $\S \S 1629$ II 3, 1796 BGB in Betracht.

\section{Ergebnis}

Steht dem Minderjährigen ein Zeugnisverweigerungsrecht zu, so wird es wegen der Höchstpersönlichkeit dieses Rechts stets vom Betroffenen selbst ausgeübt.

Eine Einschränkung der eigenen Entscheidungsbefugnis des Minderjährigen ist nur dort gerechtfertigt, wo dem Zeugen die notwendige Einsichtsfähigkeit fehlt, den Konflikt zwischen Aussage, Aussagepflicht und eigenen

\footnotetext{
${ }^{112}$ MünchKomm-Hinz, § 1680 Rn 1, 7; Soergel-Strätz, § 1629 Rn 26, 38.
} 
Interessen, derentwegen die Zivilprozeßordnung ein Zeugnisverweigerungsrecht einräumt, zu begreifen. Der Schutz des Minderjährigen gebietet hier, den aussagebereiten Zeugen nur dann einzuvernehmen, wenn die gesetzlichen Vertreter, also im Regelfall die Eltern, dem zustimmen. Soweit das Sorgerecht nur einem Elternteil zusteht, genügt dessen Zustimmung. Der nicht aussagebereite Zeuge darf, ungeachtet der subjektiven Motivation für die Weigerung, auch mit Zustimmung der Eltern nicht gegen seinen Willen vernommen werden.

Die Zustimmungsbefugnis der Eltern entfällt, wenn beide Elternteile oder auch nur einer von ihnen selbst an dem Verfahren beteiligt ist, in dem der Jugendliche aussagen soll. Hier muß analog den §1629 II 1, $\S 1795$ I Nr. 3 BGB ein Ergänzungspfleger bestellt werden, sobald die Aussagebereitschaft des nicht einsichtsfähigen Zeugen festgestellt wird. Die Entscheidung des Pflegers tritt dann an die der Eltern; ein Aussagezwang wird auch hier nicht begründet.

Die Erklärung der Ausübung des Zeugnisverweigerungsrechts gem. § 386 I ZPO erfolgt beim einsichtsfähigen Minderjährigen durch diesen selbst; allerdings ist eine Vornahme durch den gesetzlichen Vertreter mangels Höchstpersönlichkeit der Erklärungshandlung möglich. Beim nicht einsichtsfähigen Zeugen erfolgt die Erklärung durch die Eltern oder bei deren Ausschluß durch den Pfleger. Die Bestellung eines Pflegers nur für die Erklärung und die Glaubhaftmachung des Zeugnisverweigerungsrechtes kann erforderlich sein, wenn aufgrund konkreter Umstände eine Gefährdung der Interessen des Kindes zu befürchten ist, also auch wenn die Eltern am Verfahren nicht beteiligt sind oder wenn der Minderjährige von Anfang an nicht aussagebereit ist. 


\title{
$\S 7$ Die Belehrung des minderjährigen Zeugen
}

\author{
A. Gesetzliche Regelung
}

\section{Zweck der Belehrung}

Nach § 383 II ZPO ist ein Zeuge, der wegen seiner engen verwandtschaftlichen oder persönlichen Beziehungen zu mindestens einer der Parteien die Aussage verweigern darf, über sein Zeugnisverweigerungsrecht $\mathrm{zu}$ belehren. Die Pflicht zur Belehrung steht nicht zur Dispostion der Parteien, des Gerichts oder des Zeugen; es handelt sich um eine zwingende Vorschrift.

Durch die Belehrung soll dem in der Regel rechtsunkundigen Zeugen der Grund und der Umfang seines Weigerungsrechtes deutlich gemacht werden. Nur die Kenntnis seines Rechts ermöglicht ihm eine verantwortliche Entscheidung durch die Abwägung des Für und Wider einer Aussage ${ }^{1}$.

In den übrigen Fällen berechtigter Zeugnisverweigerung, also den § 383 I Nr. 4 bis $6, \S 384$ ZPO ist keine Belehrung vorgeschrieben. Da aber auch bei dem hier angesprochenen weigerungsberechtigten Personenkreis nicht ohne weiteres ausreichende Rechtskenntnisse unterstellt werden können, ist eine Belehrung des Zeugen auch ohne gesetzliche Anordnung als zulässig zu erachten ${ }^{2}$. Davon ist die Frage zu trennen, ob das Unterlassen einer zwar sachgerechten und zweckmäßigen, aber rechtlich nicht vorgeschriebenen

\footnotetext{
${ }^{1}$ Baumbach-Lauterbach-Hartmann, § 383 Anm. 4.

${ }^{2}$ Ebenda (Fn 1), § 384 Anm. 1; E. Peters, ZZP 76, 145, 162 Fn 77; Thomas-Putzo, § 384 Anm. 1; Stein-Jonas-Schumann, § 383 Rn 103; ebenda § 384 Rn 19; Zöller-Stephan, § 384 Rn 1.
} 
Belehrung gegebenenfalls der Verwertung einer Zeugenaussage entgegenstehen könnte ${ }^{3}$.

Für den prozeß- und prozeßhandlungsunfähigen Zeugen fehlt eine spezielle Regelung über die Belehrung ebenso, wie das Gesetz zur Folge einer fehlerhaften Belehrung schweigt. Bei einem Minderjährigen ist daran zu denken, da $ß$ die besondere Schutzbedürftigkeit eine Belehrung auch in den durch § 383 II ZPO nicht erfaßten Fällen gebietet. Hier ist insbesondere an das Aussageverweigerungsrecht nach $\S 384$ Ziff. 1 ZPO zu denken ist. Die Belehrung ist dort mehr als nur "nobile officium" des Gerichts ${ }^{4}$, auch wenn eine Unterlassung keinen Gesetzesverstoß im Sinne des $\S 550 \mathrm{ZPO}^{5}$ darstellt und damit kein Rechtsmittel zu begründen vermag. So kann das Gericht aufgrund seiner prozessualen Fürsorgepflicht gegenüber dem Zeugen zur Belehrung verpflichtet sein, wenn sich zu Beginn oder während der Vernehmung Anhaltspunkte ergeben, daß die Voraussetzungen eines Aussageverweigerungsrechtes nach $\S 384 \mathrm{ZPO}$ vorliegen ${ }^{6}$.

\section{Belehrung des Ausübungsbefugten}

\section{Belehrung des Minderjährigen selbst}

Grundsätzlich ist der Zeuge selbst zu belehren, da er nur so in die Lage versetzt wird, die höchstpersönliche Entscheidung über die Ausübung des Zeugnisverweigerungsrechtes zu treffen. Bei einem genügend Einsichtsfähigen reicht daher regelmäßig dessen alleinige Belehrung aus, da er auch allein entscheidet ${ }^{7}$.

\footnotetext{
${ }^{3}$ Zur Verwertbarkeit einer Zeugenaussage bei Mängeln der Beweiserhebung unten $\S 9$.

${ }^{4}$ Gottwald, BB 1979, 1780, 1781.

${ }^{5}$ OLG Köln, RPfleger 1985, 494; Stein-Jonas-Schumann, § 383 Rn 103.

${ }^{6}$ Musielak-Stadler, Rn 86. Für den Bereich des $\S 53$ StPO, der ebenfalls keine Belehrungspflicht enthält, ist anerkannt, $\mathrm{da} ß$ das Gericht bei offensichtlicher Unkenntnis des Zeugen von seinem Weigerungsrecht aufgrund seiner Fürsorgepflicht zur Belehrung verpflichtet ist, vgl. Kleinknecht/Meyer, § $53 \mathrm{Rn} 44$; Löwe-Rosenberg-Dahs, § 53 Rn 59.

${ }^{7}$ Stein-Jonas-Schumann, § $383 \mathrm{Rn} 101 ;$ Löwe-Rosenberg-Dahs, § $52 \mathrm{Rn} 45$. Zur Gleichstellung mit erwachsenen Zeugen oben $\S 6$ B.
} 
Bei fehlender Einsichtsfähigkeit sollte nach der früheren Rechtsprechung des Reichs- und des Bundesgerichtshofes die Belehrung der gesetzlichen Vertreter ausreichen ${ }^{8}$. Dies war jedoch nur die Konsequenz der Ansicht, die die gesetzlichen Vertreter allein über die Ausübung des Zeugnisverweigerungsrechtes entscheiden lassen wollte und daher für die Belehrung auch des Minderjährigen keinen Anlaß sah'. Nachdem nun anerkannt ist, daß auch der nicht Einsichtsfähige nicht gegen seinen Willen zur Aussage gezwungen werden kann, ist die alleinige Belehrung der gesetzlichen Vertreter nicht mehr ausreichend ${ }^{10}$. Geht man wie hier davon aus, daß der Minderjährige unabhängig von seinen geistigen Fähigkeiten immer selbst zur Rechtsausübung befugt ist und deshalb keine Vertretung vorliegt, ergibt sich schon daraus die Notwendigkeit der Belehrung des Minderjährigen selbst ${ }^{11}$.

Als praktische Erwägung für diese Ansicht mag sprechen, daß die Belehrung des Zeugen, sofern sie nicht bereits schriftlich mit der Ladung, sondern erst während der mündlichen Verhandlung erfolgt, geeignet ist, dem Gericht die Prüfung der Einsichtsfähigkeit überhaupt erst zu ermöglichen. Der Richter kann während der Belehrung durch geeignete Fragen feststellen, ob der Zeuge sie versteht ${ }^{12}$. Daraus ergibt sich im übrigen auch, daß in der Regel erst der Zeuge selbst und nur im Anschluß daran gegebenenfalls die gesetzlichen Vertreter zu belehren sind. Die Belehrung nach § 377 III, IV ZPO ist dagegen unzweckmäßig, es sei denn, man belehrt die Eltern immer vorsorglich über ihr Zustimmungsrecht.

${ }^{8}$ BGHSt 12, 235, 242; Roestel, RdJB 1971, 218, 219. Bereits Schimmack, JW 1924, 1667 war der Meinung, daß "bei der Belehrung von Kindern über das Zeugnisverweigerungsrecht meist wenig oder nichts herauskommt." Die Konsequenz Schimmacks, auf die Belehrung der gesetzlichen Vertreter überhaupt zu verzichten, ist nach heutiger Auffassung mit dem Minderjährigenschutz unvereinbar. Zum Zivilprozeß heute noch Bruns, Rn $185 \mathrm{~d}$.

${ }^{9}$ Bosch, S. 49 stellt darauf ab, daß das Gericht darauf vertrauen dürfe, daß die gesetzlichen Vertreter das Für und Wider einer Aussage abgewogen haben.

${ }^{10}$ BGHSt 21, 303, 306; 23, 221, 223; NJW 1979, 1722; NStZ 1991, 295 = MDR 1991, 553; ebenfalls zum Strafprozeß Denger, ZRP 1991, 48, 50. Zur Entwicklung im Strafprozeß oben $\$ 5$ B III. Nach der Änderung des $\$ 52$ II, III StPO kann die frühere Ansicht nicht mehr aufrecht erhalten werden.

${ }^{11}$ So im Ergebnis auch Baumbach-Lauterbach-Hartmann, Einf. vor §§ 383-389 Anm. 3; Grundmann, S. 94; Stein-Jonas-Schumann, § 383 Rn 102; OLG Stuttgart, FamRZ 1965, 515, 516.

${ }^{12}$ Orlowsky, S. 171. 


\section{Belehrung der gesetzlichen Vertreter} bei fehlender Einsichtsfähigkeit des Zeugen

Anders ist die Rechtslage bei einem nicht ausreichend einsichtsfähigen Minderjährigen zu beurteilen. Dieser bedarf zu einem wirksamen Verzicht auf sein Zeugnisverweigerungsrecht stets der Zustimmung der gesetzlichen Vertreter; bei deren Ausschluß muß der Ergänzungspfleger sein Einverständnis zur Vernehmung erklären ${ }^{13}$. Die Verweigerung der Zustimmung zur Aussage des aussagebereiten Jugendlichen kommt der Zeugnisverweigerung nahe, ohne damit identisch zu sein, weil dieselbe Rechtsfolge eintritt, nämlich die Befreiung des Minderjährigen von seinen Zeugenpflichten.

Das Zustimmungserfordernis beruht auf der besonderen Schutzbedürftigkeit gerade des nicht einsichtsfähigen Jugendlichen. Nur durch die Belehrung der zustimmungsbefugten Personen ist gewährleistet, daß die Interessen des aussagebereiten, aber weigerungsberechtigten Jugendlichen durch sachgerechte Überlegungen gewahrt werden ${ }^{14}$.

Das Recht zur Verweigerung der Eidesleistung ist hinsichtlich der Belehrung entsprechend den dargelegten Grundsätzen zu behandeln. Eine Belehrung der gesetzlichen Vertreter ist aber dann entbehrlich, wenn die Beeidigung wegen fehlender Eidesfähigkeit sowieso zu unterbleiben hat ${ }^{15}$.

\footnotetext{
${ }^{13}$ Dazu oben $\S 6 \mathrm{C}$ III. Im folgenden ist nur von den gesetzlichen Vertretern oder den Eltern die Rede, da für einen nach Maßgabe der oben dargestellten Grundsätze zu bestellenden Ergänzungspfleger jeweils entsprechendes gilt.

${ }^{14} B G H$, NJW 1979, 1722 zu § 52 III StPO; Stein-Jonas-Schumann, § 383 Rn 102.

${ }^{15}$ Zur Eidespflicht s.o. § 5 A III; zum Eidesverweigerungsrecht § 5 B I 2.
} 


\section{B. Umfang der Belehrung}

Nach dem Gesagten ergeben sich hinsichtlich des Umfanges der Belehrung des jugendlichen Zeugen keine Besonderheiten, soweit dieser dem volljährigen Zeugen gleichgestellt ist ${ }^{16}$.

Bei der Belehrung eines noch nicht einsichtsfähigen Zeugen ist jedoch der geistige Entwicklungsstand des Jugendlichen angemessen zu berücksichtigen und auf die Verständnismöglichkeit abzuheben ${ }^{17}$. Neben der Belehrung über das Recht zur Aussageverweigerung ist der Minderjährige darauf hinzuweisen, daß die bloße Zustimmung der gesetzlichen Vertreter ihn allein nicht zur Aussage verpflichtet ${ }^{18}$. Es ist daher deutlich zu machen, daß der Minderjährige bei gegebenem Weigerungsgrund, der hier praktisch nur auf verwandschaftlichen Beziehungen im Sinne des § 383 I Nr. 3 ZPO beruhen kann, keinesfalls zur Aussage verpflichtet ist. Der Gefahr, daß der Zeuge sich durch eine Einverständniserklärung der Eltern irrig zur Aussage verpflichtet sieht, obwohl er anfänglich nicht aussagebereit war, wird begegnet, wenn die Verstandesreife und die Aussagebereitschaft des Kindes vor jeder Einbeziehung der gesetzlichen Vertreter festgestellt werden. Die Belehrung über das Zeugnisverweigerungsrecht trotz Zustimmung der Eltern behält ihren Sinn dann hauptsächlich für Fälle, in denen die Aussagebereitschaft erst später im Lauf der Vernehmung wegfällt.

Ist die Zustimmung der gesetzlichen Vertreter erforderlich, sind diese über ihr Zustimmungsverweigerungsrecht zu belehren. Inhaltliche Abweichungen in der Belehrung sind wegen der Nähe zur Zeugnisverweigerung selbst nicht geboten. Dies gilt entsprechend, soweit ein Ergänzungspfleger bestellt wurde.

\footnotetext{
${ }^{16}$ Stein-Jonas-Schumann, § $383 \mathrm{Rn} 101$.

${ }^{17}$ Baumbach-Lauterbach-Hartmann, § 383 Anm. 4.

${ }^{18}$ BGHSt 21, 303, 305; NStZ 1984, 43; NStZ 1991, 295 = MDR 1991,553BaumbachLauterbach-Hartmann, Einf. vor §§ 387-389 Anm. 3; Schneider, JurBüro 1970, 1021; SteinJonas-Schumann, § $383 \mathrm{Rn} 102$.
} 



\section{§ 8 Die Vernehmung des Minderjährigen durch das Gericht}

\section{A. Prozessuale Fürsorgepflicht des Gerichts}

Der Zeuge ist der einzige Verfahrensbeteiligte im weiteren Sinne, der echten Rechtspflichten unterliegt ${ }^{1}$. Die Parteien treffen lediglich Obliegenheiten, deren Verletzung zwar Rechtsnachteile nach sich ziehen, deren Erfüllung aber nicht erzwungen werden kann.

Dem besonderen Pflichtenstatus des Zeugen entspricht eine besondere prozessuale Fürsorgepflicht des Gerichts ${ }^{2}$. Sie findet im Gesetz etwa in Aufklärungs- und Belehrungspflichten Ausdruck, beschränkt sich aber nicht darauf. Unabhängig von dem Bestehen oder der Ausübung eines Zeugnisverweigerungsrechtes ist der Zeuge in der Vernehmung der Gefahr entwürdigender Behandlung durch das Gericht, der Parteien oder deren Vertreter ausgesetzt ${ }^{3}$, der sich gerade ein minderjähriger Zeuge kaum entziehen kann.

Die Fürsorgepflicht verpflichtet das Gericht, Eingriffe in das Persönlichkeitsrecht des Zeugen zu unterlassen und solche der Parteien zu unterbinden $^{4}$. Im folgenden soll der Frage nachgegangen werden, wie ein minder-

\footnotetext{
${ }^{1}$ Bosch, DRiZ 1951, 107, 108; Stein-Jonas-Schumann, Einl. Rn 236; ebenda, Vor § 373 Rn 33.

${ }^{2}$ Der Begriff der Fürsorgepflicht des Gerichts findet vorwiegend in der strafprozessualen Literatur Verwendung, vgl. etwa Kleinknecht/Meyer, Einl. Rn 155 ff; KMR-Paulus, § 241a Rn 2, wonach § 241a StPO eine Konkretisierung der Fürsorgepflicht gegenüber besonders schutzwürdigen Zeugen darstellt; dazu auch unten $\S 8$ C II. K. Peters, Strafprozeß, $\$ 42$ IV 2 spricht von einem "Recht auf angemessene Behandlung" des Zeugen. Im Zivilprozeß $B$ ist sie aber ebenfalls anerkannt, so etwa bei Bettermann, ZZP 91, 365, 390 (zu $\S 139$ ZPO); MusielakStadler, Rn 86 (zu § 384 ZPO); Stein-Jonas-Schumann, Vor § 373 Rn 42. Das BVerfG nimmt ausdrücklich eine Fürsorgepflicht des Gerichts an, deren Grundlage das Rechtsstaatsprinzip und das rechtliche Gehör nach Art. 20 III, Art. 103 I GG ist, BVerfGE 52, 131, 154.

${ }^{3}$ Stein-Jonas-Schumann, Vor $\S 373 \mathrm{Rn} 35$.

${ }^{4}$ Verletzungen des Persönlichkeitsrechts und der Ehre drohen insbesondere, wenn über die
} 
jähriger Zeuge über ein bestehendes Zeugnisverweigerungsrecht hinaus durch die Gewährleistung eines fairen Verfahrens ${ }^{5}$ geschützt werden kann.

\section{B. Die Nichtöffentlichkeit des Verfahrens}

\section{Ausschluß der Öffentlichkeit}

\section{Voraussetzungen und prozessualer Anspruch auf Ausschließung}

Nach § 169 Satz 1, §§ 170, 171 GVG sind die Verhandlungen vor dem Prozeßgericht mit Ausnahme von Familien-, Kindschafts- und Entmündigungssachen grundsätzlich öffentlich ${ }^{6}$. Das Gericht kann aber, sofern die Öffentlichkeit in den genannten Ausnahmefällen nicht schon kraft Gesetzes ausgeschlossen ist, das Publikum nach $\S 172$ Nr. 4, § 171b GVG ausschließen, um Jugendliche zu schützen.

Voraussetzung eines entsprechenden gerichtlichen Beschlusses ist entweder, daß der Zeuge zur Zeit der Vernehmung das 16. Lebensjahr noch nicht vollendet hat oder daß das Interesse des Zeugen am Schutz seines persönlichen Lebensbereiches vorrangig gegenüber dem Interesse der Öffentlichkeit ist. Unerheblich ist dagegen, ob durch den Ausschluß der Öffentlichkeit eine größere Gewähr der Richtigkeit der Aussage erwartet werden kann; $\S 172$ Nr. 4, § 171b GVG schützen allein den zu Vernehmenden ${ }^{7}$ vor Eingrif-

Glaubwürdigkeit des Zeugen gestritten wird; dementsprechend hat das Gericht in diesen Situationen eine erhöhte Fürsorgepflicht, Stein-Jonas-Schumann, § $395 \mathrm{Rn} 3$.

${ }^{5}$ Das Gebot des fairen Verfahrens gilt nicht nur für die Parteien, sondern auch für sonstige Beteiligte und hier insbesondere für Zeugen, BVerfGE 38,105, 112; 52, 131, 154 . Zur Bedeutung dieses Grundsatzes für den Zivilprozeß allgemein Stein-Jonas-Leipold, Vor § 128 Rn 65 ff. Leipold spricht von einer verfassungsrechtlichen Generalklausel, die die Durchführung des Verfahrens gestaltet. Ferner Schumann, ZZP 96, 137, 166 ff zur Bedeutung für die Parteien.

${ }^{6}$ Vgl. dazu auch Art. 6 Abs. I MRK BGBI. 1952 II/S. 685 und Art. 90 Bay. Verf.; RosenbergSchwab, § 23 IV. Kritisch zum Öffentlichkeitsgrundsatz Grunsky, Grundlagen, S. 224.

${ }^{7}$ Dazu Musielak-Stadler, Rn 96; Zöller-Gummer, § 172 GVG Rn 13. Der Gesichtspunkt, daß 
fen in seinen persönlichen Lebensbereich ${ }^{8}$. Dem Gericht steht bei der Beurteilung der Frage, ob diese Gefahr besteht und die Voraussetzungen der Ausschließung vorliegen, ein Beurteilungsspielraum zu, der nicht überprüfbar ist.

Die Parteien haben keinen Anspruch auf eine bestimmte Entscheidung des Gerichts, da ihr Schutz durch die genannten Vorschriften des Gerichtsverfassungsgesetzes nicht bezweckt ist. Demgegenüber hat der betroffene Zeuge einen prozessualen Anspruch auf Ausschluß der Öffentlichkeit, wenn die gesetzlichen Voraussetzungen vorliegen ${ }^{9}$. Dies ergibt sich für den Schutz des Persönlichkeitsrechts auch aus §171b II GVG, wonach die Öffentlichkeit bei überwiegendem Geheimhaltungsinteresse auf Antrag des Betroffenen ausgeschlossen werden muß $\beta^{10}$. Der Anspruch hat aber nur die ermessensfehlerfreie Prüfung durch das Gericht zum Inhalt.

Die Geltendmachung des Anspruchs ist nicht an das Vorliegen eines Zeugnisverweigerungsrechtes gebunden ${ }^{11}$. Die Nichtöffentlichkeit soll jeden Zeugen schützen; bei Beschränkung des geschützten Personenkreises auf weigerungsberechtigte Zeugen liefe der Anspruch weitgehend leer, denn mit

die Gegenwart fremder Personen die Aussagebereitschaft kindlicher Zeugen hemmt und der Ausschluß der Offentlichkeit in diesen Fällen überhaupt erst die Vernehmung ermöglicht, kann dabei nicht übersehen werden. Maßgeblich für die Entscheidung des Gerichts muß aber der Schutz des Zeugen, nicht das mittelbare Beweisinteresse der Parteien bleiben. Zum Ganzen aus vernehmungspschologischer Sicht Arntzen, Vernehmungspsychologie, S. 34 ff; Römer, RdJB 1971, 205 ff. Für das Strafverfahren wird auch die Meinung vertreten, die Aussageunwilligkeit eines Zeugen vor der Öffentlichkeit stelle eine Gefährdung der öffentlichen Ordnung dar und rechtfertige daher den Ausschluß nach $\S 172 \mathrm{Nr} .1 \mathrm{GVG}$, so RGSt 30, 244, 245 f; 64, 385, 386; Dippel, FS f. Tröndle, S. 599, 615. Dagegen BGHSt 9, 280, 283; Kleinknecht/Meyer, § 172 GVG Rn 6.

${ }^{8}$ Zöller-Gummer, § 171b GVG Rn 4 ff.

${ }^{9}$ Grunsky, Grundlagen, S. 225; Wieczorek, § 172 GVG Anm. B I d. Die gegenteilige Auffassung von Baumbach-Lauterbach-Albers, § 172 GVG Anm. 2 dürfte nach der Einführung des $\S 171$ b I 2, II GVG nicht mehr aufrecht zu erhalten sein. Diese Vorschrift räumt den Betroffenen eine weitgehende Dispositionsbefugnis über die Anwesenheit der Öffentlichkeit ein, Rieß/Hilger, NStZ 1987, 204, 207 f.

${ }^{10} \S 171 \mathrm{~b}$ GVG wurde durch das Opferschutzgesetz BGBI. 1886 I 2496 mit Wirkung zum 1.4.1987 aufgenommen. Die Vorschrift soll den Schutz aller Prozeßbeteiligten vor Bloßstellung stärken, Baumbach-Lauterbach-Albers, §171b GVG Anm. 1. Zum Opferschutzgesetz, das in seinen Auswirkungen nicht auf das Strafverfahren beschränkt ist, auch Weigend, NJW 1987, 1170 ff; Rieß/Hilger, NStZ 1987, 145 ff und 204 ff.

${ }^{11}$ Nach Wieczorek, § 172 GVG Anm. B II a können Zeugen nur über ihr Zeugnisverweigerungsrecht den Ausschließungsanspruch geltend machen. 
der Ausübung des Weigerungsrechtes wird die Gefährdung der schutzwürdigen Interessen des Zeugen in der Regel entfallen.

\section{Die Beantragung durch den minderjährigen Zeugen}

Der Minderjährige kann den Antrag nach § 171b II GVG selbst stellen. Auf die Einsichtsfähigkeit und Verstandesreife kommt es nicht an. Die Zustimmung der gesetzlichen Vertreter aus Gründen des Minderjährigenschutzes ist nicht erforderlich, da der Ausschluß der Öffentlichkeit keine negativen Auswirkungen zu Lasten des Zeugen erwarten läßt.

\section{Antragsrecht der gesetzlichen Vertreter}

Von der Zustimmungsbedürftigkeit ist die Frage zu unterscheiden, ob die gesetzlichen Vertreter selbst den Antrag stellen können, wenn sie weder Partei noch Zeuge sind.

$\mathrm{Da}$ es sich nicht um eine höchstpersönliche Entscheidung handelt, steht einem Antrag auf Ausschluß der Öffentlichkeit durch die Eltern nichts entgegen. Grundlage des Antragsrechts ist das Personensorgerecht ${ }^{12}$. Die für die Ausübung des Zeugnisverweigerungsrechts durch Erklärung gegenüber dem Gericht entwickelten Grundsätze können hier entsprechend herangezogen werden ${ }^{13}$. Da das Prozeßgericht die Möglichkeit hat, die Öffentlichkeit von Amts wegen auszuschließen, wird allerdings eine Pflegerbestellung nur für den Antrag nach $\S 171 \mathrm{~b}$ GVG nicht in Betracht kommen.

\section{Antragsrecht der am Verfahren beteiligten Sorgeberechtigten}

Die gesetzlichen Vertreter können den Antrag auf Ausschluß der Öffentlichkeit nicht für das Kind stellen, da ihnen das Personensorgerecht durch die Bestellung eines Ergänzungspflegers auch insoweit entzogen wird ${ }^{14}$.

\footnotetext{
${ }^{12}$ Wieczorek, ebenda.

${ }^{13}$ Oben $\S 6 \mathrm{D}$.

${ }^{14} \mathrm{Vgl}$. oben $\S 6 \mathrm{C}$; die dort entwickelten Grundsätze gelten bei Beteiligung mindestens eines Elternteiles stets, weil dann immer auch das Zeugnisverweigerungsrecht des Angehörigen
} 
Auswirkungen hat der Ausschluß der Eltern jedoch nicht, weil sie nicht gehindert sind, im eigenen Namen zu handeln.

\section{Anwesenheitsrecht der nicht am Verfahren beteiligten gesetzlichen Vertreter}

Der Ausschluß betrifft stets die gesamte Öffentlichkeit ${ }^{15}$, da es mit dem Grundsatz der Öffentlichkeit des Verfahrens nicht zu vereinbaren wäre, nur bestimmten Personen oder Personengruppen den Zutritt zu versagen oder zu gewähren ${ }^{16}$. Eine Anordnung des Gerichts, die Öffentlichkeit mit Ausnahme der gesetzlichen Vertreter auszuschließen, wäre daher in dieser Form nicht zulässig.

Gleichwohl kann die Anwesenheit der Eltern bei der Vernehmung erforderlich sein, etwa wenn ihre Zustimmung zur Aussage des Kindes erforderlich ist oder die Anwesenheit die Aussagebereitschaft des Zeugen fördert. Das Gericht kann den Eltern die Anwesenheit in nichtöffentlicher Verhandlung, mithin also nach Ausschluß der Öffentlichkeit, durch besonderen Beschluß gemäß § 175 II 1 GVG gestatten ${ }^{17}$.

\section{Ausschluß der Parteien}

Die Parteien sind grundsätzlich berechtigt, an der Beweisaufnahme teilzunehmen, §357 I ZPO ${ }^{18}$. Von einem Ausschluß der Öffentlichkeit gemäß $\S 170$ ff GVG sind sie nicht betroffen; eine Ausnahme stellt die Entfernung als sitzungspolizeiliche Maßnahme nach § 177 GVG dar. Das Anwesenheitsrecht der Parteien gilt in jedem Stadium des Verfahrens und

nach § 383 I Nr. 3 ZPO gegeben ist. Zum Umfang der Entziehung des Sorgerechts vgl. unten $\S 12 \mathrm{~A}$.

${ }^{15}$ Zum Ausschluß der Parteien s.u. § 8 B II.

${ }^{16}$ Zöller-Gummer, $\$ 169$ GVG Rn 5. Eine Ausnahme gilt nur für sitzungspolizeiliche Maßnahmen aufgrund der $\S 175$ ff GVG.

${ }^{17}$ Nach Baumbach-Lauterbach-Albers, § 175 GVG Anm. 3 entscheidet das Gericht in "ganz freiem Ermessen". Dem kann nicht gefolgt werden, weil das Gericht eine Abwägung der Interessen des Zeugen und der der Öffentlichkeit vorzunehmen hat; diese Abwägung erfolgt im pflichtgemäßen Ermessen. Vgl. auch Stein-Jonas-Leipold, Vor § $128 \mathrm{Rn} 67$, der darauf hinweist, daß nur die pflichtgemäße Ermessensausübung verfahrensfehlerfrei sein kann.

${ }^{18}$ Zur Bedeutung des Anwesenheitsrechts Baumbach-Lauterbach-Hartmann, § 357 Anm. 1; Stein-Jonas-Schumann, § $357 \mathrm{Rn} 1 \mathrm{ff}$. 
dient der Verwirklichung des rechtlichen Gehörs ${ }^{19}$. Es ermöglicht den Parteien, durch Fragen, Einwände und Vorhaltungen Einfluß auf die Beweisaufnahme und damit auch auf das Beweisergebnis zu nehmen. Die Verletzung dieses Rechts, das in den $\$ \S 285,357$ ZPO Ausdruck gefunden hat, führt zur Unverwertbarkeit des so ermittelten Beweisergebnisses ${ }^{20}$.

Unter besonderen Umständen kann jedoch die Anwesenheit der Parteien während der Vernehmung mit schutzwürdigen Interessen des Zeugen kollidieren. Diese Gefahr besteht bei einem minderjährigen Zeugen insbesondere, wenn die Eltern selbst Partei des Verfahrens sind; ihre bloße Anwesenheit kann negativen Einfluß auf das Kind haben und die Aussage verfälschen $^{21}$. Im Strafverfahren ist in $\S 247$ Satz 2 StPO die Möglichkeit vorgesehen, den Angeklagten während der Vernehmung eines noch nicht sechzehnjährigen Zeugen aus dem Sitzungssaal zu entfernen. Die Vorschrift dient dem Schutz des kindlichen Zeugen.

Die Zivilprozeßordnung kennt keine entsprechende Vorschrift. Nachdem eine Gefährdung des Kindeswohls aber auch im Zivilverfahren nicht ausgeschlossen werden kann, mithin eine vergleichbare Situation besteht, ist die Gesetzeslücke durch die analoge Anwendung des § 247 StPO zu schließen ${ }^{22}$. Der Ausschluß einer oder beider Parteien ist nur zum Schutz des Zeugen, nicht zur Erzielung eines "richtigen" Beweisergebnisses zulässig ${ }^{23}$.

\footnotetext{
${ }^{19}$ Waldner, S. 74.

${ }^{20}$ Stein-Jonas-Leipold, $\$ 285 \mathrm{Rn} 1$. Eine Verletzung des rechtlichen Gehörs liegt auch bei einem vor der Beweisaufnahme erklärten Verzicht des Beweisführèrs vor, weil dies mit der Rechtsstaatlichkeit des Verfahrens nicht vereinbar wäre. Ein nachträglicher Verzicht und Heilung gemäß § 295 I ZPO ist damit jedoch nicht ausgeschlossen, Baumgärtel, FS für Habscheid, S. 1, 6 f; Stein-Jonas-Leipold, Vor § 128 Rn 51.

${ }^{21}$ Arntzen, Vernehmungspsychologie, S. $37 \mathrm{f}$ empfiehlt, Kinder nicht in Gegenwart der Eltern über Sexualdelikte zu vernehmen, um Hemmungen des Zeugen abzubauen. Im Zivilprozeß sind solche Hemmungen weniger zu erwarten, wenn es um Beweisthemen geht, die das Kind nicht in seinem Schamgefühl betreffen. Es kann daher nur im Einzelfall entschieden werden, ob die Eltern im Interesse des Kindes während der Vernehmung anwesend sein sollen. Ähnlich zum vormundschaftsgerichtlichen Verfahren Jansen, § 12 Rn 97; vgl. dazu auch unten $\S 11$.

${ }^{22}$ Ebenso Stein-Jonas-Schumann, § 357 Rn 22; Wieczorek, § 357 Anm. B III; Zöller-Stephan, § 357 Rn 5. Zweifelnd Baumbach-Lauterbach-Hartmann, §357 Anm. 1 A, der die $\S 177 \mathrm{ff}$ GVG als abschliessende Regelung ansieht.

${ }^{23}$ Kleinknecht/Meyer, § 247 Rn 11; KMR-Paulus, § 247 Rn 17; Löwe-Rosenberg-Gollwitzer, § 247 Rn 23; Weigend, NJW 1987, 1170, 1171 f. Auch Art. 6 I 2 MRK nennt die Interessen von Jugendlichen als zulässigen Ausschließungsgrund. Die Vorschrift ist auch für das Zivilverfahren anwendbar, Kleinknecht/Meyer, Rn 1 zu Art. 6 MRK (Anhang A4).
} 
Das Gericht entscheidet über den Ausschluß der Parteien nach pflichtgemäßem Ermessen. Angesichts der hohen Bedeutung des Anwesenheitsrechts für die Parteien sind konkrete Anhaltspunkte für eine erhebliche $\mathrm{Be}$ einträchtigung oder Gefährdung des physischen oder psychischen Wohls des Kindes zu fordern ${ }^{24}$. Der alleinige Umstand, daß die Eltern Prozeßpartei sind, rechtfertigt die Annahme einer hinreichenden Gefährdung des Kindeswohls selbst dann nicht, wenn der Zeuge vom Prozeßgegner benannt wurde und mit einer für die Eltern ungünstigen Aussage gerechnet werden muß. Auch hier müssen zusätzliche Verdachtsmomente hinzutreten. $\mathrm{Zu}$ denken wäre insbesondere an die Bedrohung oder Einschüchterung des Kindes oder den Versuch, den Zeugen zu einer unrichtigen Aussage zu veranlassen.

Nach Abschluß der Vernehmung sind die gesetzlichen Vertreter analog $\S 247$ Satz 2 StPO wieder zuzulassen und über den Inhalt der Aussage zu unterrichten $^{25}$.

\section{Durchführung der Vernehmung}

\section{Allgemeines}

Nach §395 I ZPO beginnt die Vernehmung mit der Ermahnung des Zeugen zur Wahrheit und bei Eidesfähigkeit im Sinne des § 393 ZPO mit dem Hinweis auf die Möglichkeit der Vereidigung. Soweit ein Zeugnisverweigerungsrecht besteht oder im Laufe der Vernehmung ein Aussageverweigerungsrecht in Betracht kommt, ist der Zeuge zu belehren ${ }^{26}$. Um die Erforderlichkeit der Beteiligung der gesetzlichen Vertreter und gegebenenfalls der Anrufung des Vormundschaftsgerichtes feststellen zu können, muß das Prozeßgericht vor der Vernehmung zur Sache die Aussagebereitschaft und die geistige Reife prüfen ${ }^{27}$. Erst danach kann die eigentliche Verneh-

\footnotetext{
${ }^{24}$ Ähnlich zum Strafverfahren Kleinknecht/Meyer, § 247 Rn 11.

${ }^{25}$ Stein-Jonas-Schumann, § 357 Rn 22; Zöller-Stephan, § 357 Rn 5.

${ }^{26}$ Zur Belehrung oben $\S 7$.
} 
mung, zunächst zur Person und im Anschluß daran zum Beweisthema durchgeführt werden, §§ 396, 397 ZPO.

Die Vernehmung soll einerseits das für die Entscheidung des Rechtsstreits relevante Wissen des Zeugen ausschöpfen ${ }^{28}$, ohne ihn auf der anderen Seite zu überfordern oder in seiner personalen Würde zu verletzen ${ }^{29}$. Dazu gehört auch die strikte Vermeidung einer dem kindlichen Alter des Zeugen nicht angemessenen Sprache ${ }^{30}$ und das Stellen von diskriminierenden, einschüchternden oder suggestiven Fragen ${ }^{31}$. Die allgemeinen Erkenntnisse der Vernehmungspsychologie sind zu berücksichtigen, wobei für die Einzelheiten auf die einschlägige Literatur verwiesen werden kann ${ }^{32}$. Im vorliegenden Zusammenhang soll lediglich auf zwei Fragen eingegangen werden, die die Rechtsstellung des minderjährigen Zeugen unmittelbar berühren.

\section{Das Fragerecht der Parteien}

Den Parteien steht nach Maßgabe des § 397 II ZPO das Recht zu, mit Erlaubnis des Vorsitzenden Richters selbst unmittelbar Fragen an den Zeugen zu richten ${ }^{33}$. Die Gestattung unmittelbarer Fragen durch die Parteien steht im pflichtgemäßen Ermessen des Gerichts; das Fragerecht der Prozeßbevollmächtigten besteht ohne diese Einschränkung.

\footnotetext{
${ }^{27} \mathrm{Vgl}$. zur Zustimmungsbefugnis der gesetzlichen Vertreter und der Möglichkeit der nachträglichen Genehmigung oben § 6 C II 1.

${ }^{28}$ Baumbach-Lauterbach-Hartmann, §286 Anm. 3 A a; ebenda, §397 Anm. 2; ZöllerStephan, § $397 \mathrm{Rn} 4$.

${ }^{29}$ BVerfGE 38, 105, $114 \mathrm{f}$; zur Bedeutung der Grundrechte des Zeugen und einer "verfassungsnahen" Zeugenvernehmung Stein-Jonas-Schumann, Vor $\S 373$ Rn 35 m.w.N.; v. Lanzenauer, DRiZ 1966, 223, 224.

${ }^{30}$ Michaelis, in: Arntzen, Vernehmungspsychologie, S. 43; Müller-Luckmann, RdJB 1971, 217 f; Musielak-Stadler, Rn 93.

${ }^{31}$ Zur besonderen Beeinflussbarkeit kindlicher Zeugen bereits Stern, DJZ 1908, $51,53 \mathrm{f}$.

${ }^{32} \mathrm{Vgl}$. dazu etwa Arntzen, Psychologie der Zeugenaussage; derselbe, Vernehmungspsychologie; Krönig, S. 31 ff; Müller-Luckmann, RdJB 1971, 217 f; K. Peters, Strafprozeß, § 44 mit zahlreichen Nachweisen; Schneider, Beweis und Beweiswürdigung3, insbesondere S. $166 \mathrm{ff}, 178 \mathrm{ff}$, 181 ff; weitere Nachweise bei Stein-Jonas-Schumann, Vor § 373 Rn 1; ebenda, § 396 Rn 1.

${ }^{33}$ Stein-Jonas-Schumann, § $397 \mathrm{Rn} 2$.
} 
Im Strafverfahren ist das Fragerecht des Angeklagten und seiner Verteidiger demgegenüber eingeschränkt. Nach § 241a StPO darf ein Zeuge, der das sechzehnte Lebensjahr noch nicht vollendet hat, nur vom Vorsitzenden Richter befragt werden, es sei denn, ein Nachteil für das Wohl des Zeugen ist durch eine direkte Befragung nicht zu befürchten.

Vorrangiger Zweck der genannten Regelung ist der größtmögliche Schutz kindlicher und jugendlicher Zeugen vor den Belastungen einer gerichtlichen Vernehmung ${ }^{34}$. Da dieses Ziel auch im Zivilverfahren gilt, andererseits die Zivilprozeßordnung keine Vorschriften enthält, die den Besonderheiten Rechnung tragen, die sich bei der Vernehmung Minderjähriger ergeben können, kommt m.E. die entsprechende Anwendung des § 241a StPO in Betracht. Eine unverhältnismäßige Einschränkung der Rechte der Parteien ist damit nicht verbunden, weil die Möglichkeit unangetastet bleibt, dem Zeugen Fragen über das Gericht vorlegen zu lassen. Auf der anderen Seite wird die Gefahr einer negativen Beeinflussung und Gefährdung des Kindeswohles verringert, weil das Prozeßgericht auf diese Weise Fragen nach Form und Inhalt kontrollieren und sie gegebenfalls nur in abgewandelter Form zulassen kann $^{35}$.

\footnotetext{
${ }^{34}$ Kleinknecht/Meyer, § 241a Rn 1; KMR-Paulus, § 241a Rn 2; Löwe-Rosenberg-Gollwitzer, $\S 241 \mathrm{a}$ Rn 1 .

${ }^{35}$ Die Frage, ob das Gericht die Vorlegung einer Frage wegen ihres Inhaltes im Rahmen des § 241a StPO verweigern kann, ist in der strafrechtlichen Literatur umstritten. Es wird vertreten, die Vorschrift lasse lediglich die Anpassung der Frage in eine dem Alter des Zeugen angemessene Form zu, KMR-Paulus, §241a Rn6. Aus der Verweisung des § 241a II StPO auf § 241 II StPO ergibt sich aber die Zurückweisungsmöglichkeit bezüglich aller ungeeigneten Fragen; ungeeignet sind Fragen, die nach Form oder Inhalt dem Entwicklungsstand des jugendlichen Zeugen nicht entsprechen, Löwe-Rosenberg-Gollwitzer, § 241a $\mathrm{Rn} 2 \mathrm{ff}, 5$. Für den Zivilprozeß ist die Entscheidungsbefugnis des Prozeßgerichts in $\S 397$ III ZPO normiert. Nach dieser Vorschrift sind unzulässige Fragen abzulehnen, BaumbachLauterbach-Hartmann, §397 Anm. 2; Stein-Jonas-Schumann, §397 Rn 8; v. Lanzenauer, DRiZ 1966, 223, 224; Zöller-Stephan, § 397 Rn 4. Unzulässig sind nach Form oder Inhalt zur Klärung der Beweisfrage ungeeignete Fragen.
} 


\section{Wiederholte Vernehmung}

Eine weitere Frage betrifft die Zulässigkeit wiederholter oder nachträglicher Vernehmungen über dasselbe Beweisthema gemäß §398 ZPO ${ }^{36}$. Grundsätzlich soll die Aussage in einer einmaligen Vernehmung gewonnen und fixiert werden. Jede Wiederholung der Vernehmung stellt eine besondere Belastung des Zeugen dar, die ihm nur ausnahmsweise zugemutet werden darf ${ }^{37}$. Eine Ausnahme ist gegeben, wenn die erste Vernehmung infolge verfahrensfehlerhafter Durchführung nicht verwertbar is $\mathrm{t}^{38}$.

Die Frage der fehlerfreien Neuvornahme kann deswegen nicht ohne weiteres bejaht werden, weil jede weitere Vernehmung eine zusätzliche Belastung des minderjährigen Zeugen darstellt ${ }^{39}$. Das Verwertungsverbot, das dem Schutz des Zeugen dient, wirkt sich bei unbeschränkter Zulässigkeit der wiederholten Vernehmung entgegen seiner Zweckbestimmung zum Nachteil des Zeugen aus. Hier besteht ein Konflikt zwischen Zeugenschutz und Beweisinteressen der Parteien.

Jede neuerliche Vernehmung des Zeugen gemäß § 398 ZPO setzt einen besonderen Anlaß voraus ${ }^{40}$. Ausreichend ist neben der Unverwertbarkeit der Aussage die Erwartung, ein Zeuge wolle seine Aussage ändern oder verzichte nun auf sein Zeugnisverweigerungsrecht ${ }^{41}$.

\footnotetext{
${ }^{36}$ Eine wiederholte Vernehmung liegt nur vor, wenn sich die erneute Vernehmung auf dasselbe Beweisthema bezieht. Auf die Zeugenvernehmung in der Berufung ist nicht einzugehen, da die Minderjährigkeit des Zeugen hier keine Besonderheiten gegenüber dem erstinstanzlichen Verfahren aufwirft.

${ }^{37}$ Vgl. zum Strafverfahren Nr. 19 I RiStBV, wonach die mehrmalige Vernehmung von Kindern und Jugendlichen wegen der damit verbundenen seelischen Belastung dieser Zeugen nach Möglichkeit zu vermeiden ist. Dazu auch Denger, ZRP 1991, 48, 49 f; Dippel, FS f. Tröndle, S. 599, 609; Römer, RdJB 1971m 205, 207; Stern, DJZ 1910, 1002, 1003.

${ }^{38}$ Stein-Jonas-Schumann, § $398 \mathrm{Rn} 2$ und 5; Zöller-Stephan, § $398 \mathrm{Rn} 5$. Im einzelnen dazu $\S 9$.

${ }^{39}$ Römer, RdJB 1971, 205, 207 weist zum Strafverfahren darauf hin, daß vielfache Befragungen und Vernehmungen bei einem Kind häufig mehr Schaden anrichten, als das deliktische Geschehen gegenüber dem Kind selbst. Er fordert daher zutreffend die Reduzierung der Zahl der Vernehmungen und deren "Komprimierung" auf das unbedingt notwendige Maß; ebenso Denger, ZRP 1991, 48, 49 f.

${ }^{40}$ Stein-Jonas-Schumann, Einl. Rn 254; ebenda, § 398 Rn 5.

${ }^{41}$ Baumbach-Lauterbach-Hartmann, § 398 Anm. 2 b, aa; Schneider, S. 194; Wieczorek, § 398 Anm. B II b; Zöller-Stephan, § 398 Rn 5.
} 
Diese Grundsätze gelten auch für die wiederholte Vernehmung eines minderjährigen Zeugen. Nachdem das Gericht durch die erste Vernehmung bereits zu erkennen gegeben hat, daß es den Zeugen für geeignet hält, besteht für eine Beweiseinschränkung durch Zulassung nur einer einmaligen Vernehmung keine gesetzliche Grundlage. Soweit sich der Beweisführer darauf beruft, der Minderjährige oder die zustimmungsbefugten Eltern seien jetzt aussage- oder zustimmungsbereit, hat er dies näher darzulegen ${ }^{42}$. Die Anforderungen an die Darlegungslast sollten im Interesse des Minderjährigenschutzes nicht zu gering angesetzt werden ${ }^{43}$. Die bloße Behauptung eines Sinneswandels genügt nicht, zumal die Aussagebereitschaft eines Kindes bei gerichtlicher Vernehmung jederzeit wieder entfallen kann.

\section{Ergebnis}

Der Schutz insbesondere minderjähriger Zeugen ist durch die Gewährung von Zeugnis- und Aussageverweigerungsrechten im Einzelfall nur unzureichend sichergestellt. Das Gericht ist zur Fürsorge verpflichtet. Das bedeutet insbesondere, den Zeugen vor Beeinträchtigungen seines grundrechtlich geschützten Persönlichkeitsrechtes zu bewahren und ein faires Verfahren zu gewährleisten.

Als Schutzmaßnahmen kommen bei Zeugen vor Vollendung des sechzehnten Lebensjahres der Ausschluß der Öffentlichkeit und der Parteien in Betracht; § 247 Satz 2 StPO findet entsprechende Anwendung. Den gesetzlichen Vertretern kann die Anwesenheit gestattet werden, wenn dies im Interesse des Kindes liegt.

Die Durchführung der Vernehmung obliegt entsprechend § 241a StPO dem Vorsitzenden des Prozeßgerichts oder dem Einzelrichter. Die unmittelbare Befragung durch die Parteien oder deren Bevollmächtigte ist nur zuzulassen, wenn Gefährdungen des Kindeswohls nicht zu befürchten sind.

\footnotetext{
${ }^{42}$ Zur Darlegungslast bei angeblich unvollständiger erster Vernehmung OLG Schleswig, OLGZ 1980, 58, 59.

${ }^{43}$ Die erhöhte Darlegungslast findet ihre Entsprechung in den höheren Anforderungen bei einem Beweisantrag auf Vernehmung eines noch nicht sechs Jahre alten Zeugen; vgl. oben § 3 B III 2 c.
} 
Eine wiederholte Vernehmung ist nach Maßgabe des § 398 ZPO zulässig. Die Partei, die die erneute Vernehmung beantragt, ist gehalten, den Anlaß dafür im Einzelnen darzulegen. 


\section{§ 9 Die Verwertung einer prozeßordnungswidrig gewonnenen Aussage}

\section{A. Abgrenzung}

Eine Zeugenaussage kann, wie jedes der gesetzlich vorgesehenen Beweismittel, materiell rechtswidrig oder unter Verletzung prozessualer Vorschriften gewonnen werden. In beiden Fällen stellt sich die Frage, ob die Aussage zur Urteilsfindung herangezogen werden darf. Gleichwohl ist zwischen beiden Möglichkeiten zu unterscheiden, was in der Literatur häufig nicht beachtet wird; dort wird vorwiegend die materiell-rechtliche Rechtswidrigkeit behandelt ${ }^{1}$. Diese kann beim Zeugenbeweis etwa gegeben sein, wenn der Zeuge durch seine Aussage gegen bürgerlich-rechtliche oder strafrechtliche Vorschriften verstößt oder die Aussage selbst durch rechtswidrige Nötigung des Zeugen beeinflußt wird².

Die Stellung und der Schutz des minderjährigen Zeugen werden allein durch seine prozessualen Rechte und Pflichten bestimmt. Im vorliegenden Zusammenhang ist daher nur auf die Verletzung prozessualer Vorschriften oder eine sonst fehlerhafte Beweisaufnahme und ihre Folgen einzugehen.

\footnotetext{
${ }^{1}$ Vgl. etwa Dilcher, AcP 158, 469 ff, 497 f; Habscheid, FS für Peters, S. 840, 854 ff m.w.N.; Kaissis, S. 8 ff; E. Peters, ZZP 76, 145 ff; Wais, S. 84 ff; Werner, NJW 1988, 993 ff.

${ }^{2}$ Dazu Musielak-Stadler, Rn 32 f; Wais, S. 109 f.
} 


\section{B. Die fehlerhafte Belehrung}

\section{Darstellung}

Die gesetzliche Belehrungspflicht ${ }^{3}$ ist verletzt, wenn der Zeuge oder die Zustimmungsbefugten nicht oder nicht vollständig über ihr Recht zur Zeugnis- bzw. Zustimmungsverweigerung aufgeklärt werden ${ }^{4}$. Dies kann insbesondere dann von Bedeutung sein, wenn sich erst während der Vernehmung herausstellt, daß die Verstandesreife des jugendlichen Zeugen doch nicht für eine selbständige Entscheidung hinreicht. Hier müssen sowohl die gesetzlichen Vertreter ${ }^{5}$ als auch der Minderjährige noch einmal belehrt werden.

\section{Verfahrensrechtliche Folgen der Falschbelehrung}

\section{Nachholung der Belehrung}

Die fehlerhafte Zeugenbelehrung stellt einen Mangel des Beweisverfahrens dar. Er kann beseitigt werden, indem die Belehrung nachgeholt wird

\footnotetext{
${ }^{3} \mathrm{Im}$ folgenden geht es nur um die Belehrung nach $\$ 383$ II ZPO. Die fehlerhafte Belehrung in den übrigen Fällen aufgrund der Fürsorgepflicht stellt keinen Gesetzesverstoß im Sinne des $\S 550 \mathrm{ZPO}$ dar. Die Aussage ist in diesen Fällen daher stets verwertbar; OLG Köln, RPfleger 1985, 494; Stein-Jonas-Schumann, § 383 Rn 103. Anders Wais, S. 100, der eine Verletzung der richterlichen Hinweispflicht gemäß § 139 ZPO annimmt. Zur Belehrung selbst vgl. oben § 7.

${ }^{4}$ Eine fehlerhafte Belehrung liegt daher immer dann vor, wenn das Gericht den Ausschluß der gesetzlichen Vertreter analog § 1629 II 1, § 1795 I Nr. 3 BGB verkennt. Die Belehrungspflicht bezieht sich auch auf den in diesen Fällen zu bestellenden Ergänzungspfleger. Zur Falschbelehrung vgl. BGH bei Dallinger, MDR 1974, 16; Habscheid, ZZP 96, 306, 320; Waldner, S. 68 f. Zur StPO Kleinknecht/Meyer, § 52 Rn 32.

${ }^{5}$ Orlowsky, S. 181 ist der Auffassung, die Verletzung der Mitwirkungsbefugnisse der gesetzlichen Vertreter sei nicht revisibel, da sie nur dem Schutz des Zeugen, nicht des Angeklagten dienen. Anders Löwe-Rosenberg-Dahs, §52 Rn 56. Auf den Zivilprozeß kann diese Argumentation nicht übertragen werden.
} 
und der Zeuge an seiner einmal gemachten Aussage festhält ${ }^{6}$. Entsprechendes gilt, wenn die gesetzlichen Vertreter nachträglich auf ihr Recht zur $\mathrm{Zu}$ stimmungsverweigerung verzichten und auch das Kind weiterhin aussagebereit ist? ${ }^{7}$.

Umgekehrt darf die Aussage nicht mehr verwertet werden, wenn der Zeuge oder die Eltern nach der Belehrung erklären, nunmehr von ihrem Weigerungsrecht Gebrauch machen zu wollen. Dieser Fall ist der vorherigen Belehrung gleichzustellen ${ }^{8}$.

\section{Verwertungsverbot}

Das unter Verletzung zwingender Verfahrensvorschriften gewonnene Beweisergebnis darf, sofern keine fehlerfreie Neuvornahme stattgefunden hat, für die Entscheidungsfindung nicht herangezogen werden. Es entspricht allgemeiner Überzeugung, daß die Aussage des nicht ordnungsgemäß belehrten Zeugen unverwertbar ist ${ }^{9}$.

Diese Rechtsfolge ergibt sich aus dem Verbot der Beeinflussung des Zeugen in seiner Entschließungsfreiheit ${ }^{10}$. Für das Strafverfahren folgt das aus § 136a, § 69 III StPO; es handelt sich um allgemeine Rechtsgedanken, die auch auf den Zivilprozeß anwendbar sind ${ }^{11}$. Unerheblich ist, ob die feh-

\footnotetext{
${ }^{6}$ BGH, NJW 1985, 1470 f; E. Peters, ZZP 76, 145, 161 Fn 72; Wieczorek, § 383 Anm. D I c.

${ }^{7} \mathrm{Vgl}$. oben $\$ 6$ C II $1 \mathrm{c}$.

${ }^{8}$ Dies gilt auch für das Strafverfahren, Kleinknecht/Meyer, § 52 Rn 32; Löwe-Rosenberg-Dahs, $\S 52$ Rn 52 f; ebenda, Schäfer, Einl. Kapitel 14 Rn 22 ff; K. Peters, Gutachten, S. 115; E. Schmidt, JR 1959, 369, 372. Zum Zivilprozeß vgl. die nachfolgend Genannten.
}

${ }^{9}$ BGH, NJW 1985, 1158, 1159 = VersR 1984, 458 f; NJW 1985, 1470 f; Baumbach-Lauterbach-Hartmann, aa0, § 383 Anm. 6; Bruns, Rn 185 c; Habscheid, ZZP 96, 306, 320; E. Peters, ZZP 76, 145, 160 Fn 66; Rosenberg-Schwab, § 113 IV 2; Stein-Jonas-Leipold, § 284 Rn 18; ebenda, Schumann, § 383 Rn 100; Thomas-Putzo, § 295 Anm. 1 a; ebenda, § 383 Anm. 4; Wais, S. 94 ff; Wieczorek, § 383 Anm. D I c; Zöller-Stephan, § 383 Rn 21.

\footnotetext{
${ }^{10}$ Nach $R G S t$ 48, 269, 270; 57, 63, 65 führt die falsche Belehrung des Zeugen im Strafverfahren nur dann zur Unverwertbarkeit, wenn die Entschließungsfreiheit tatsächlich eingeschränkt wurde. Im Zweifel muß aber zum Schutz des Zeugen von einer Beeinflussung ausgegangen werden; BayObLGZ 56, 389, 391; Baumbach-Lauterbach-Hartmann, § 383 Anm. 6; Niese, S. 146; E. Peters, ZZP 76, 145, 160 ff sieht den Grund, neben dem Schutz der Familienbande, auch in der Vermeidung falscher Zeugnisse; K. Peters, Gutachten, S. 127; Wais, S. 113.

${ }^{11}$ Maunz in Maunz-Dürig, Art. 2 Rn 34 ff weist darauf hin, daß jede Täuschung des Zeugen
} 
lerhafte Belehrung wissentlich erteilt wurde, denn das Verwertungsverbot tritt auch bei unvorsätzlicher Falschbelehrung ein ${ }^{12}$.

\section{Heilung des Verfahrensfehlers}

a) Parteiherrschaft und Prozeßökonomie

Die Parteien des Zivilprozesses bestimmen über die tatsächlichen Grundlagen des Rechtsstreites. Sie können auf Beweismittel verzichten oder bereits gestellte Beweisanträge zurücknehmen. Dem trägt § 295 I ZPO Rechnung, indem hinsichtlich Verfahrensvorschriften im Parteiinteresse Verletzungsfolgen nur eintreten, wenn die Parteien nicht auf die Einhaltung dieser Vorschriften verzichten ${ }^{13}$. Zu den Verfahrensvorschriften gehören auch die Regelungen über die Durchführung der Beweisaufnahme einschließlich der Belehrung von Zeugen.

Neben dem Gedanken des Verzichts durch die Parteien soll § 295 I ZPO den schnellen und sicheren Prozeßgang verwirklichen und Prozeßökonomie fördern ${ }^{14}$. Die Parteien sind aufgrund der Rügeobliegenheit gehalten, ihnen bekannte oder infolge Fahrlässigkeit unbekannt gebliebene Fehler bis zur nächsten mündlichen Verhandlung geltend zu machen.

zugleich dessen Persönlichkeitsrecht verletzt. Im Ergebnis ebenso Bruns, Rn 185 d, wenn der Zeuge fälschlich belehrt wird, ihm stehe kein Zeugnisverweigerungsrecht zu.

${ }^{12}$ Kleinknecht/Meyer, § $52 \mathrm{Rn} 35$; ebenda, § 136a Rn 13 f; Löwe-Rosenberg-Hanack, § 136a $\mathrm{Rn} 42$.

${ }^{13} B G H$, NJW 1964, 449, 450; Hagen, JZ 1972, 505, 509; Musielak-Stadler, Rn 58; Stein-JonasLeipold, §295 Rn 1. Gemeint ist stets der nachträgliche Verzicht, Baumgärtel, FS für Habscheid, S. 1, 6 f.

${ }^{14}$ BGH, NJW 1985, 1158, 1159 = VersR 1984, 458 f; Baumbach-Lauterbach-Hartmann, § 295 Anm. $2 \mathrm{C}$ a. 
b) Rügeverzicht und Zeugenschutz

Die hier nur kurz dargestellte Ansicht stellt die Einhaltung der zeugenschützenden Verfahrensgewährleistungen zur Disposition der Parteien.

Die unrichtige Belehrung erschwert es dem Zeugen bis hin zu einer ungewollten Nichtausübung, eine verantwortliche Entscheidung über sein Zeugnisverweigerungsrecht zu treffen. Die Folgen sind mit der Vernehmung nicht beendet, sondern setzen sich mit der Verwertung der Aussage for $\mathrm{t}^{15}$. Die Verwertung wird durch Rügeverzicht und -unterlassung, also durch prozessuales Verhalten der Parteien ermöglicht. Angesichts der großen Bedeutung, die dem Schutz des Persönlichkeitsrechts des Zeugen zukommt ${ }^{16}$, erscheint zumindest zweifelhaft, ob Parteiherrschaft und Prozeßökonomie eine hinreichende Grundlage für die weitergehende Belastung des Zeugen bieten können.

Das Bundesverfassungsgericht hat in seiner Entscheidung vom 8.10.1974 ${ }^{17}$ darauf hingewiesen, daß es mit Art. 2 I, Art. 1 I GG nicht vereinbar sei, den Zeugen zum bloßen Objekt des Verfahrens zu machen. Im einzelnen führt es aus: "Das Recht des Zeugen, in dem Konflikt zwischen Aussage- und Wahrheitspflicht und der Gefahr eigener Belastung bestimmte Angaben zu verweigern, wird in Frage gestellt, wenn man ihn auf die vom Ermessen anderer Verfahrensbeteiligter abhängige Belehrung verweisen und auf seine laienhafte Entscheidung ohne sachverständige Hilfe beschränken, den im allgemeinen rechtsunkundigen Zeugen also letztlich den Reaktionen anderer Verfahrensbeteiligter ausliefern würde." Der grundrechtlich geschützte Persönlichkeitsbereich des Zeugen sei Einwirkungen durch das Verfahrensrecht und seiner Anwendung durch die Verfahrensbeteiligten entzogen.

\footnotetext{
${ }^{15}$ Denger, ZRP 1991, 48 ff; Habscheid, ZZP 96, 306, 320; derselbe, FS für Peters, S. 840, 858 ff; E. Peters, ZZP 76, 145, 161.

${ }^{16}$ Die Zeugnisverweigerungsrechte beruhen auf einer Abwägung verfassungsrechtlich geschützter Interessen zugunsten des Zeugen. Im Gegenzug wird das rechtliche Gehör der Parteien eingeschränkt; BVerfGE 33, 367, 375 (zu § 53 StPO); Schmidt-Aßmann in Maunz-Dürig, Art. 19 Abs. IV Rn 225.
}

${ }^{17} B V$ erfGE 38, 105, 114 f. Die Entscheidung betrifft Verfassungsbeschwerden gegen Beschlüsse des Bundesdisziplinargerichts, durch die Rechtsbeiständen von Zeugen die Anwesenheit während der Vernehmung verweigert wurde. Die Feststellungen des BVerfG zur Rechtsstellung des Zeugen im Verhältnis zu anderen Verfahrensbeteiligten sind jedoch von den Besonderheiten dieses Verfahrens unabhängig und daher verallgemeinerungsfähig. 
c) Stellungnahme

Eine verfassungskonforme Auslegung des Prozeßrechts kann bei $\mathrm{Zu}-$ grundelegung der Rechtsprechung des Bundesverfassungsgerichtes im Rahmen des § 295 I ZPO die mittelbar durch ein Verhalten der Parteien drohenden Einwirkungen zu Lasten des Zeugen nicht außer acht lassen. So darf der Zeugen nicht nur als bloßes Beweismittel gesehen werden, weil so die Gefahr besteht, ihn als bloßes Objekt des Verfahrens zu behandeln.

Der verfassungsrechtlich garantierte Schutz des Zeugen schließt aber nicht aus, diesem Schutz dienende prozessuale Regelungen der Herrschaft der Parteien zu unterwerfen. Unter § 295 II ZPO fallen nur Vorschriften, die ausschließlich im Interesse der Öffentlichkeit liegen ${ }^{18}$. Das allgemeine Interesse an einer ordnungsgemäßen Durchführung des Verfahrens genügt nicht, da sonst nahezu jeder Verfahrensverstoß unheilbar wäre.

Das Prozeßrecht legt die Kontrolle über die Einhaltung der im jeweiligen Parteiinteresse liegenden Vorschriften in die Hand der Beteiligten und belastet sie zugleich mit der Rügeobliegenheit. Die Verfahrensvorschriften sollen nicht um ihrer selbst willen eingehalten werden, sondern nur soweit dies im Interesse der Parteien und zur Findung einer sachgerechten Entscheidung erforderlich ist ${ }^{19}$. Die Heilung setzt den Rügeverzicht beider Parteien voraus ${ }^{20}$ und bietet allein dadurch die Gewähr einer zweckmäßigen Verfahrensdurchführung. Der Schutz des Zeugen findet nur mittelbar statt. Dies genügt aber, weil der Persönlichkeitsschutz des Zeugen nicht dazu führen kann, ihm eine über seine prozessuale Funktion hinausgehende Einflußmöglichkeit auf das Verfahren einzuräumen.

\footnotetext{
${ }^{18}$ Baumbach-Lauterbach-Hartmann, § 295 Anm. 3 A; Baumgärtel, ZZP 69, 89, 111; MusielakStadler, Rn 58; Rosenberg-Schwab, § 68 I 2; Stein-Jonas-Leipold, § 295 Rn 4; Zöller-Stephan, $\S 295 \mathrm{Rn} 4$.

${ }^{19} \mathrm{Vgl}$. Schmidt-A,3mann in Maunz-Dürig, Art. 19 Abs. IV Rn $240 \mathrm{f}$ zur Verfassungsmäßigkeit der von ihm als "Rügemodell" bezeichneten Fehlerfolge; Zöller-Stephan, § $295 \mathrm{Rn} 1$; ebenda, $\S 296 \mathrm{Rn} 2$.

${ }^{20}$ Es handelt sich um einen vom Gericht begangenen Verfahrensfehler, den beide Parteien rügen können, Baumbach-Lauterbach-Hartmann, aa0, § 295 Anm. 2 A; Rosenberg-Schwab, $\S 68$ II 1; Stein-Jonas-Leipold, § 295 Rn 1, 18.
} 


\section{Verkennung der Einsichtsfähigkeit und der Zustimmungsbefugnis}

Die Feststellung der Verstandesreife und die Einholung der Zustimmung der gesetzlichen Vertreter dienen dem Schutz des minderjährigen Zeugen. Die Schutzbedürftigkeit ist umso mehr gegeben, je weniger die Fähigkeit vorliegt, selbständige Entscheidungen treffen zu können.

Verkennt das Gericht die Einsichtsfähigkeit oder hält es beide oder einen Elternteil zu Unrecht für befugt, dem Verzicht des Kindes auf sein Zeugnisverweigerungsrecht zuzustimmen, ist die so gewonnene Aussage nicht verwertbar. Wie bei der unrichtigen Belehrung kann der Verfahrensfehler aber durch Neuvornahme oder nach Maßgabe des § 295 I ZPO geheilt werden.

Heilung und Rügeverzicht der Parteien sind nicht erforderlich, wenn das Prozeßgericht zu Unrecht von einem Ausschluß der Eltern ausgeht und einen Ergänzungspfleger bestellen läßt ${ }^{21}$. Die Bestellung durch das Vormundschaftsgericht schränkt das elterliche Sorgerecht im Umfang der auf den Pfleger gemäß $\$ 1630$ I BGB übertragenen Aufgaben auch dann ein, wenn die Bestellung rechtswidrig ist ${ }^{22}$. Auswirkungen auf den Zivilprozeß sind daher mit dem Eingriff in das elterliche Sorgerecht nicht verbunden ${ }^{23}$.

\section{Verletzung der Vorschriften über die Öffentlichkeit des Verfahrens}

Der Zeuge hat gemäß § 171b II GVG einen prozessualen Anspruch auf Ausschluß der Öffentlichkeit, wenn die Vernehmung Umstände betrifft, die zum persönlichen Lebensbereich des Aussagepflichtigen gehören ${ }^{24}$. Umgekehrt kann der Zeuge aber auch der Ausschließung der Öffentlichkeit widersprechen, § 171b I 2 GVG.

\footnotetext{
${ }^{21}$ Dazu im Einzelnen unten $\S 11$.

${ }^{22}$ BGHZ 33, 195, 201; MünchKomm-Goerke, § $1909 \mathrm{Rn} 55$. Etwas anderes gilt nur, wenn die Vormundschaftsgerichtliche Entscheidung ausnahmsweise nicht nur rechtswidrig, sondern nichtig ist.

${ }^{23}$ Nach $B G H Z$ 41, 303, 309 f gebietet nur die Rücksichtnahme auf die Belange des Pfleglings die Herbeiführung einer korrigierenden Entscheidung des Vormundschaftsgerichts.

${ }^{24} \mathrm{Vgl}$. oben $\S 8 \mathrm{~B}$.
} 
Der rechtswidrige Ausschluß der Öffentlichkeit wird von der h.M. als wesentlicher Verfahrensmangel angesehen, der nicht dem Rügeverzicht des § 295 I ZPO zugänglich ist ${ }^{25}$. Dem ist zuzustimmen, weil es sich bei den betreffenden Vorschriften des Gerichtsverfassungsgesetzes nicht um Normen handelt, die das Interesse der Parteien, sondern öffentliche Interessen schützen sollen ${ }^{26}$. Es ist daher nur ergänzend darauf hinzuweisen, daß bereits die Verletzung prozessualer Rechte des Zeugen dieses Ergebnis rechtfertigt, ohne daß es eines Rückgriffs auf das öffentliche Interesse bedarf. Da die Vorschriften über die Öffentlichkeit des Verfahrens aber auch dann verletzt sind, wenn die Voraussetzungen der $\S 171 \mathrm{~b}, 174$ GVG verkannt wurden, ohne daß überhaupt interessierte Zuhörer anwesend waren ${ }^{27}$, liegt immer sowohl eine Verletzung der Interessen der Öffentlichkeit als auch des Zeugen vor.

Die gerichtliche Entscheidung, die das fehlerhafte Verfahren abschließt, ist mit den Rechtsmitteln der Berufung und der Revision angreifbar. Eine Beschwerdemöglichkeit Dritter oder eines Zeugen kennt das Gesetz nicht. Der Schutz des Zeugen bei einer Verletzung seines Anspruch auf nicht-öffentliche Vernehmung ist nicht mit einer Sanktionsandrohung belegt, wenn die Prozeßparteien auf die Einlegung eines Rechtsmittels verzichten. Der Verzicht hätte dann eine ähnliche Wirkung wie ein unzulässiger Rügeverzicht.

Die fehlende Rügemöglichkeit des Zeugen verletzt diesen nicht in seinem Anspruch auf rechtliches Gehör ${ }^{28}$. Nach § 174 I GVG muß über den Öffentlichkeitsausschluß mündlich verhandelt werden; dabei sind die Beteiligten anzuhören. Beteiligte im Sinne des § 174 I GVG sind nicht nur die Prozeßparteien, sondern auch der betroffene Zeuge ${ }^{29}$. Die dem Zeugen damit ein-

\footnotetext{
${ }^{25}$ Bruns, Rn 163 b; Stein-Jonas-Leipold, §295 Rn 6; ebenda, Vor $\S 128 \mathrm{Rn} 130$. Aus der Rechtsprechung RGZ 157, 341, 347. Anders Zöller-Stephan, § $295 \mathrm{Rn} 5$, nach dem nur der vorherige Verzicht auf die Öffentlichkeit unwirksam sei.

${ }^{26}$ Die fehlerhafte Behandlung der Parteiöffentlichkeit stellt demgegenüber einen verzichtbaren Verfahrensmangel dar, weil keine öffentlichen Interessen betroffen sind; $B G H, \mathrm{LM} \mathrm{Nr}$. $7 \mathrm{zu} \S 295 \mathrm{ZPO}$.

${ }^{27}$ Stein-Jonas-Leipold, Vor $§ 128 \mathrm{Rn} 130$.

${ }^{28}$ Das OLG Stuttgart, ZZP 70, 382, $383 \mathrm{f}$ ließ die Frage offen, ob einem Rechtsanwalt ein eigenes Beschwerderecht bei fehlerhaftem Ausschluß der Öffentlichkeit zusteht, weil es nur Berufung und Revision als statthaftes Rechtsmittel ansah.
}

${ }^{29}$ Baumbach-Lauterbach-Albers, §174 GVG Anm. 1 A; Zöller-Gummer, § 174 GVG Rn 1 
geräumte Gelegenheit zur Stellungnahme genügt den Anforderungen an das rechtliche Gehör ${ }^{30}$. Der Ausschluß der Anfechtbarkeit der Entscheidung des Gerichts nach § 171b III GVG ist daher nicht zu beanstanden.

\section{Ergebnis}

Die Verletzung von Minderjährigenschutzvorschriften oder zeugenschützenden Regeln führt stets zur Unverwertbarkeit der Aussage. Dazu gehören die unterlassene oder unrichtige Belehrung des Zeugen und des Zustimmungsbefugten, die Nichthinzuziehung der gesetzlichen Vertreter und die verfahrenswidrige Beteiligung der Eltern trotz ihrer Verhinderung. Die rechtswidrige Bestellung eines Ergänzungspflegers hat auf die Verwertbarkeit der mit dessen Zustimmung gewonnenen Aussage keine Auswirkungen.

Der Verfahrensfehler kann durch fehlerfreie Neuvornahme oder durch Heilung im Wege des Rügeverzichts und der Rügeunterlassung nach $\S 295$ I ZPO geheilt werden. Dies gilt auch für Mängel, die zugleich das Persönlichkeitsrecht des Zeugen verletzen.

Die Verletzung des Anspruchs auf Ausschluß der Öffentlichkeit kann von dem Zeugen nicht angefochten werden. Den Parteien stehen die allgemeinen Rechtsmittel offen.

hält die formlose Anhörung der Auskunftsperson für ausreichend, wobei unklar bleibt, ob eine Anhörungspflicht seitens des Gerichts besteht.

${ }^{30} \mathrm{Vgl}$. allgemein Waldner, S. $59 \mathrm{ff}$. 



\section{§ 10 Zwischenstreit über das Vorliegen eines Zeugnisverweigerungsrechts und Rechtsmittel des Zeugen}

\section{A. Der Zwischenstreit über die Berechtigung der Zeugnisverweigerung}

\section{Gegenstand des Zwischenverfahrens}

Erklärt der Zeuge vor oder während der Vernehmung unter Angabe des Grundes ${ }^{1}, \mathrm{da} ß$ er die Aussage verweigert, kann zwischen der beweisführenden Partei und dem Zeugen ein Zwischenstreit geführt werden. Dabei stehen sich das Zeugnisverweigerungsrecht und der Anspruch der Partei auf Ablegung des Zeugnisses gegenüber ${ }^{2}$. Ziel des Verfahrens ist, die Berechtigung des Zeugen zur Aussageverweigerung aufgrund des behaupteten Grundes $^{3}$ verbindlich festzustellen. Dieselbe Situation entsteht, wenn die gesetzlichen Vertreter erklären, daß sie mit einer Vernehmung ihres minderjährigen Kindes nicht einverstanden sind.

\footnotetext{
${ }^{1}$ Der Grund der Zeugnisverweigerung muß gemäß § 386 I ZPO glaubhaft gemacht werden. Unterbleibt die Begründung gänzlich, so wird kein Zwischenstreit geführt, sondern direkt Zeugniszwang nach $\S 390 \mathrm{ZPO}$ ausgeübt, Baumbach-Lauterbach-Hartmann, §387 Anm. 1; Zöller-Stephan, § $387 \mathrm{Rn} 2$.

${ }^{2}$ So bereits $R G Z 20,378,379 \mathrm{f}$.

${ }^{3}$ Das Zwischenurteil, daß gemäß § 387 III ZPO zu erfolgen hat, enthält nur über den jeweils konkret behaupteten Weigerungsgrund eine verbindliche Entscheidung; eine Präklusion des Zeugen mit etwaigen weiteren Zeugnisverweigerungsgründen findet nicht statt, Stein-JonasSchumann, § $386 \mathrm{Rn} 6$; ebenda, § 387 Rn 4; Baumbach-Lauterbach-Hartmann, § 387 Anm. 3. Einschränkend Bosch, DRiZ 1951, 137, 138, der (beim Untersuchungsverweigerungsrecht) "in freier Handhabung der gesetzlichen Bestimmungen" nur ein einmaliges Nachschieben von Gründen für zulässig erachtet, ohne dies weiter zu begründen.
} 
Die Zuständigkeit für die Entscheidung liegt bei dem Prozeßgericht ${ }^{4}$. Erforderlich ist jedoch ein Antrag des Beweisführers ${ }^{5}$. Wird der Antrag nicht gestellt, so liegt darin regelmäßig ein Verzicht auf den Zeugen ${ }^{6}$. Nach Maßgabe des § 399 ZPO kann bei einem Verzicht des Beweisführers auch der Beweisgegner eine Entscheidung über die Zeugnisverweigerung herbeiführen, wenn der Zeuge erschienen ist. Parteien des Zwischenverfahrens sind der Beweisführer und gegebenenfalls der Beweisgegner ${ }^{7}$ auf der einen Seite, andererseits der Zeuge ${ }^{8}$.

Die Entscheidung wird gemäß § 387 III ZPO nach Anhörung der Beteiligten ${ }^{9}$ durch Zwischenurteil getroffen, gegen das der jeweils Unterlegene mit der sofortigen Beschwerde vorgehen kann ${ }^{10}$.

\footnotetext{
${ }^{4}$ Dies gilt auch bei einer Vernehmung vor dem beauftragten oder ersuchten Richter, $\S 389$ ZPO; Wieczorek, § 387 Anm. A.

${ }^{5}$ Zöller-Stephan, $\$ 387$ Rn 2. A.A. Baumbach-Lauterbach-Hartmann, §387 Anm. 2; SteinJonas-Schumann, $\S 387 \mathrm{Rn} 2$, wonach das Prozeßgericht von Amts wegen tätig wird. Dagegen spricht aber, daß bei Untätigkeit des Beweisführers, auch nach den zuletzt Genannten, aufgrund der Dispositionsmöglichkeit des Beweisführers im Zweifel ein Verzicht der Partei auf den Zeugen vorliegt und ein Zwischenstreit dann entbehrlich ist.
}

${ }^{6}$ So bereits Meyer, ZZP 17, 459, $460 \mathrm{f}$ zu $\$ 339$ CPO; $B G H$, NJW 1964, 449, 450; NJWRR 1987, 445;Niese, S. 143; Stein-Jonas-Schumann, § 387 Rn 8; Wieczorek, § 387 Anm. A II a 1; Zöller-Stephan, § 387 Rn 2. Baumbach-Lauterbach-Hartmann, §387 Anm. 1; BGH, LM Nr. 9 zu § $295 \mathrm{ZPO}$ stellen auf $\S 295$ I ZPO ab, ohne daß dies zu unterschiedlichen Ergebnissen führt.

${ }^{7}$ Bruns, Rn 185 f; Stein-Jonas-Schumann, $\$ 387$ Rn 8. Die Beteiligung des Beweisgegners hat keine Auswirkungen auf die Stellung des Zeugen im Zwischenverfahren. Im folgenden wird daher nur vom Beweisführer gesprochen.

${ }^{8}$ Bruns, ebenda (Fn 7); Baumbach-Lauterbach-Hartmann, § 387 Anm. 2; Lent, ZZP 52, 14, 24; Niese, S. 143; Wieczorek, § 387 Anm. B I.

${ }^{9} \mathrm{Da}$ die Entscheidung die Rechtsstellung des Zeugen unmittelbar berührt, ist seine Anhörung vor $\mathrm{Erla} \beta$ einer Entscheidung wegen des auch für den Zeugen gebotenen rechtlichen Gehörs notwendig, Waldner, S. 221.

${ }^{10}$ OLG Frankfurt, MDR 1983, 236 zur Beschwerde des Zeugen. 


\section{Die Durchführung des Zwischenverfahrens bei Beteiligung eines einsichtsfähigen Zeugen}

\section{Befugnis zur selbständigen Entscheidung}

Der einsichtsfähige minderjährige Zeuge kann über die Geltendmachung eines Zeugnisverweigerungsrechts selbständig entscheiden. Die gesetzlichen Vertreter haben prozessual keine Möglichkeit, den Entschluß des Jugendlichen zu kontrollieren oder abzuändern, auch wenn sie dies im Interesse des Minderjährigen für erforderlich halten ${ }^{11}$. Das Prozeßgericht ist daher auch nicht verpflichtet, die Stellungnahme der Eltern einzuholen, ob sie mit der Vernehmung des Kindes einverstanden $\operatorname{sind}^{12}$.

Es stellt sich aber die Frage, ob die Mitwirkung der gesetzlichen Vertreter nicht spätestens im Stadium des Zwischenverfahrens notwendig wird. Die Bedeutung dieser Frage ergibt sich daraus, daß der Minderjährige trotz der Verstandesreife nicht prozeßfähig im Sinne der §§ 51, 52 ZPO ist und er selbst keine Prozeßhandlungen vornehmen könnte.

\section{Die Prozeßfähigkeit Minderjähriger im Zwischenverfahren}

a) Verpflichtungsfähigkeit und prozessuale "Wehrbefugnis"

Nach § 52 ZPO liegt die Prozeßfähigkeit vor, soweit nach materiellem Recht die Fähigkeit gegeben ist, sich selbst zu verpflichten. Es handelt sich nach allgemeiner Ansicht um eine Verweisung auf die bürgerlich-rechtlichen Vorschriften über die Geschäftsfähigkeit ${ }^{13}$. Die Prozeßfähigkeit ist nicht nur eine Zulässigkeits-, sondern auch eine Prozeßhandlungsvorausset-

\footnotetext{
${ }^{11}$ Dazu im einzelnen oben $\S 6 \mathrm{~B}$.

${ }^{12}$ Die gleichwohl erklärte Zustimmungsverweigerung der gesetzlichen Vertreter ist unbeachtlich, weil bereits eine wirksame Zeugnisverweigerung vorliegt.

${ }^{13}$ Baumbach-Lauterbach-Hartmann, §52 Anm. 1; Grunsky, Grundlagen, S. 251 ff, 254; Rosenberg-Schwab, §44 I. Dagegen hält Zöller-Vollkommer, §52 Rn 2 die Verweisung des $\S 51$ ZPO für gegenstandslos, weil §52 ZPO eine eigenständige Regelung enthält. Die ZPO regelt aber die Verpflichtungsfähigkeit gerade nicht, so daß es doch des Rückgriffs auf das materielle Recht bedarf.
} 
zung $^{14}$. Die Verweisung des $\S 52 \mathrm{ZPO}$ ist insoweit zu eng, als es im vorliegenden Zusammenhang nicht um die Wahrnehmung materiellrechtlicher Gestaltungsmöglichkeiten geht, sondern um die Ausübung einer prozessualen Befugnis. Bei strikter Anwendung der genannten Vorschriften müßte auch der verstandesreife Minderjährige durch die Eltern oder einen Pfleger vertreten werden, obwohl er zur Ausübung des streitgegenständlichen Zeugnisverweigerungsrechtes gerade nicht der Mitwirkung Dritter bedarf.

Die Prozeßfähigkeit wird als verfahrensrechtliche Ausprägung bürgerlichrechtlicher Handlungsfähigkeit ${ }^{15}$ und als "Wehrbefugnis aus materiellrechtlicher Rechtsgüterzuordnung" verstanden ${ }^{16}$. Die Verpflichtungsfähigkeit verliert bei dieser Definition ihre Bedeutung, weil es auf die Handlungsbefugnis ankommt. Entscheidend ist nicht die rechtsgeschäftliche Verpflichtungsfähigkeit, sondern die Fähigkeit, ein Recht selbständig ausüben zu können ${ }^{17}$. Diese Fähigkeit kann zwar ihrerseits von einem bestimmten Alter abhängen, aber auch von anderen Voraussetzungen bestimmt sein. Ob dies der Fall ist, richtet sich nicht nach dem Entstehungsgrund, sondern dem jeweiligen Rechtsgebiet, dem das betreffende Recht zuzuordnen ist. Bei einer zivilprozessualen Befugnis kommt es daher nicht auf die Geschäftsfähigkeit im Sinne der $\S 104 \mathrm{ff}$ BGB an, wenn das Prozeßrecht eine eigene Regelung der Ausübungsbefugnis enthält.

\section{b) Entscheidungsfähigkeit einsichtsfähiger Zeugen}

Die eigenständige Geltendmachung des Zeugnisverweigerungsrechtes setzt voraus, daß der Zeuge die geistige Reife und Einsichtsfähigkeit besitzt, den Konflikt zwischen Aussagepflicht und Belastung eigener oder fremder

\footnotetext{
${ }^{14}$ Grundmann, S. 5 m.w.N.; ebenda, S. 11 f.

${ }^{15}$ BVerfGE 28, 243, 254; 51, 405, 407; Bruns, Rn 53; Rosenberg-Schwab, § 44 I spricht von prozessualer Handlungsfähigkeit. Ähnlich auch Fehnemann, S. 43 ff.

${ }^{16}$ Kahlke, ZZP 100, 10 f. Grunsky, Grundlagen, S. 253, 254 stellt unter Hinweis auf $\S 1025$ I ZPO fest, daß das Gesetz materiellrechtliche und prozessuale Verfügungsbefugnis parallel behandelt.

${ }^{17}$ Grunsky, Grundlagen, S. 252 f; Kahlke, ZZP 100, 10, 15. Zweifelnd Fehnemann, S. 47 ff, deren Bedenken gegen eine Verallgemeinerung darauf beruhen, $\mathrm{da} B$ den Eltern nicht jede Einflußmöglichkeit entzogen werden dürfe. Diese Gefahr besteht nicht, wenn den Eltern ergänzend das Recht eingeräumt wird, neben dem Kind selbst als gesetzliche Vertreter im Namen des Kindes Rechtsmittel einlegen zu können; dazu unten § 10 B III.
} 
Interessen einer nahestehenden Partei in verantwortlicher Weise zu lösen. Soweit diese Fähigkeit gegeben ist, wird der minderjährige Zeuge einem Volljährigen gleichgestellt. In einem Zwischenverfahren ist er selbst prozeßfähig ${ }^{18}$, ohne daß es der Einwilligung oder Genehmigung der gesetzlichen Vertreter bedarf. Auf die Prozeßfähigkeit im Sinne der $\S 51,52$ ZPO kommt es dagegen nicht $a^{19}$.

Im Ergebnis entspricht die Prozeßähigkeit im Zwischenverfahren damit der Ausübungsbefugnis des Zeugnisverweigerungsrechtes, das Gegenstand des Zwischenstreites is ${ }^{20}$. Die Ausübungsbefugnis enthält die Prozeß- und Prozeßhandlungsfähigkeit des Zeugen, ohne daß Dritte beteiligt werden müssen ${ }^{21}$.

Eine Beeinträchtigung des notwendigen Minderjährigenschutzes ist dadurch nicht zu befürchten. Die Verstandesreife des Zeugen befähigt ihn zur eigenverantwortlichen Entscheidung und vermindert zugleich seine Schutzbedürftigkeit. Auch hat der Gesetzgeber in anderen Fällen zu erkennen gegeben, daß trotz jugendlichen Alters eine im Verhältnis zur Regelung der $\S \S 51,52 \mathrm{ZPO}$ vorverlagerte Prozeßfähigkeit möglich ist ${ }^{22}$.

${ }^{18}$ Im Ergebnis ebenso Bosch, S. $39 \mathrm{f}$ und S. $55 \mathrm{f}$, der aber ohne Rücksicht auf die Entscheidungsreife auf die feste Altersgrenze des 14. Lebensjahres abstellt; wie hier Grundmann, S. 93; Lent, ZZP 52, 14, 24; Stein-Jonas-Schumann, § 387 Rn 2. Ferner ZöllerStephan, § 387 Rn 3, der jedoch nicht nach der Einsichtsfähigkeit differenziert.

${ }^{19}$ Zur Prozeßfähigkeit im Zusammenhang mit der Ausübung eines Zeugnisverweigerungsrechts durch Prozeßhandlung des Zeugen auch oben § 5 B I 1.

${ }^{20}$ Ähnlich wird die Fähigkeit eines Minderjährigen zur Einlegung einer Verfassungsbeschwerde mit der Grundrechtsmündigkeit, verstanden als Ausübungsbefugnis, begründet, ohne daß es auf eine - im Bundesverfassungsgerichtsgesetz nicht geregelte - Prozeßfähigkeit ankäme, BVerfGE 1, 87, 88; 51, 405, 407 f m.w.N.; NJW 1986, 3129 ff; Fehnemann, S. 51 ff; Hohm, NJW 1986, 3107, 3108 ff; Kahlke, ZZP 100, 10, 15.

${ }^{21}$ Vgl. auch oben $\S 6$ A, B. Kahlke, ZZP 100, 10, 17 f verweist darauf, daß im Streit über die Prozeßfähigkeit auch die Menschenwürde, der Anspruch auf rechtliches Gehör und der Grundsatz des fairen Verfahrens die Prozeßfähigkeit begründen können. Diese Gesichtspunkte haben über den Zulassungsstreit hinaus Bedeutung, weil es sich um allgemeine Grundsätze handelt, die in jedem Verfahren zu beachten sind. Die Prozeßfähigkeit ist eine Einschränkung der Handlungsfreiheit und nur gerechtfertigt, wo sie zum Schutz des Verfahrensbeteiligten geboten ist.

\footnotetext{
${ }^{22}$ So sind in der Geschäftsfähigkeit beschränkte Personen in Ehesachen und Eheanfechtungsverfahren gemäß $\S \S 607,640 \mathrm{~b}$ ZPO prozeßfähig. Diese Ausnahmen von dem Grundsatz, daß die Prozeßfähigkeit der Verpflichtungsfähigkeit entspricht, beruhen auf der höchstpersönlichen Natur der jeweiligen Verfahrensgegenstände, Baumbach-Lauterbach-Albers, §607 Anm. 1; Grundmann, S. 31 ff; Thomas-Putzo, § 607 Anm. 1; Zöller-Vollkommer, § 52 Rn 5.
} 


\section{Das Zwischenverfahren gegen einen nicht einsichtsfähigen Zeugen}

\section{Aussagepflicht trotz Aussageunwilligkeit oder Zustimmungsverweigerung}

Die Aussagepflicht entfällt weder durch das kindliche Alter des Zeugen, noch durch die Unfähigkeit zum wirksamen Verzicht auf ein Zeugnisverweigerungsrecht ${ }^{23}$. Dies gilt auch für die Zustimmungsverweigerung der Eltern, die nur dann prozessuale Wirkung auf die Zeugenpflichten hat, wenn das behauptete Zeugnisverweigerungsrecht tatsächlich besteht und die gesetzlichen Vertreter nicht ausgeschlossen sind.

Solange die Aussagepflicht besteht, der Zeuge aber nicht vernommen werden kann, weil er oder die Eltern sich weigern, besteht für den Beweisführer ein erhebliches Interesse an einer richterlichen Überprüfung der Rechtmäßigkeit der Verweigerung ${ }^{24}$.

Anders als beim einsichtsfähigen Minderjährigen kann die Prozeßfähigkeit hier nicht abweichend von $\S 51,52 \mathrm{ZPO}$ behandelt werden, weil der wirksame Verzicht auf das Zeugnisverweigerungsrecht von der Zustimmung der Eltern abhängt. Der Zeuge muß sich daher im Zwischenverfahren vertreten lassen. Die Vertretung ist Aufgabe der gesetzlichen Vertreter, die in Ausübung ihres Personensorgerechts handeln ${ }^{25}$. Sind die Eltern selbst am Verfahren beteiligt, sind sie insoweit von der Vertretung ausgeschlossen. Die Zuständigkeit ist dann auf den Ergänzungspfleger übergegangen, der in diesen Fällen regelmäßig vor der Vernehmung des kindlichen Zeugen zu bestellen ist ${ }^{26}$.

\footnotetext{
${ }^{23}$ Vgl. zur gegenteiligen Auffassung von K. Peters oben § 5 A II 2.

${ }^{24}$ Das Interesse des Beweisführers an einer Überprüfung besteht bei jedem Zeugen, weil die unberechtigte Zeugnisverweigerung seine Beweismöglichkeiten und damit das rechtliche Gehör im Sinne des Art. 103 I GG einschränkt. Bei kindlichen Zeugen vor Vollendung des sechsten Lebensjahres ist diese Einschränkung von besonderer Bedeutung, weil schon die Ladung des Zeugen davon abhängig ist, $\mathrm{da} B$ der Beweisführer alle anderen Beweismittel bereits ausgeschöpft hat und die Aussage des Kindes voraussichtlich prozeßentscheidend ist. Dazu im Zusammenhang mit der Zeugeneignung oben § 3 B III.

${ }^{25}$ Wieczorek, $\S 387$ Anm. B I a. Ebenso Bosch, S. 56 f allgemein für Minderjährige vor Vollendung des 14 . Lebensjahres.

${ }^{26}$ Zum Umfang der Zustimmungsbefugnis des Pflegers s.u. § 12 A.
} 
Die Durchführung des Zwischenverfahrens erscheint aber aus einem anderen Grunde problematisch. Der unreife Zeuge ist regelmäßig nicht schuldfähig, sei es, weil er das vierzehnte Lebensjahr noch nicht vollendet hat, sei es, weil er die rechtlichen Folgen seines Handeln nicht überblicken kann. Die Verhängung von Ordnungs- und Zwangsmitteln ist deshalb unzulässig, so daß die Erfüllung der Zeugenpflichten nicht erzwungen werden $k^{k a n n}{ }^{27}$. Damit stellt sich die Frage nach dem Zweck eines Zwischenverfahrens, weil die rechtskräftige Entscheidung über das Nichtbestehen eines Weigerungsrechtes ihrerseits Voraussetzung für die Ausübung von Zeugniszwang nach § 390 I, II ZPO ist.

\section{Unzulässigkeit des Zwischenverfahrens wegen fehlendem Rechtsschutzbedürfnis?}

a) Rechtsschutzbedürfnis als Verfahrensvoraussetzung

Das Rechtsschutzbedürfnis soll als Verfahrensvoraussetzung gewährleisten, daß nur solche Streitigkeiten durch die Gerichtsbarkeit entschieden werden müssen, die tatsächlich einer Entscheidung bedürfen ${ }^{28}$. Es handelt sich um eine allgemeine Zulässigkeitsvoraussetzung, die bei jeder Inanspruchnahme gerichtlicher Hilfe vorliegen muß ${ }^{29}$. Dabei ist auf die Interessen der Parteien und auf die der Allgemeinheit abzustellen ${ }^{30}$.

Das Rechtsschutzbedürfnis hat der Gesetzgeber explizit nur für Feststellungsklagen und für Klagen auf künftige Leistung in den $\S 256$ bis $259 \mathrm{ZPO}$ kodifiziert $^{31}$. Es besteht Einigkeit darüber, daß das Rechtsschutzbedürfnis

\footnotetext{
${ }^{27}$ Dazu oben $\S 4 \mathrm{C}$ III zur Verhängung von Ordnungsmitteln gegen den ordnungsgemäß geladenen Minderjährigen und $\$ 5 \mathrm{C}$ II 1 zum Zeugniszwang bei Verletzung der Aussagepflicht.

${ }^{28}$ Stein-Jonas-Schumann, Vor $\$ 253$ Rn 101; Stephan, S. 19 ff und S. 32 ff spricht von prozessualem Interesse als Bestandteil des Rechtsschutzbedürfnisses; so auch schon Schönke, S. 24.

${ }^{29}$ Baumbach-Lauterbach-Hartmann, Grundz. § 253 Anm. 5 A. Das Rechtsschutzbedürfnis ist eine allgemeine Prozeßvoraussetzung und in jedem Stadium des Verfahrens von Amts wegen zu prüfen, $B G H$, NJW 1981, 875, 876.

${ }^{30}$ Wieser, S. $30 \mathrm{ff}$.

${ }^{31}$ Das Feststellungsinteresse im Sinne der genannten Vorschriften ist ein Unterfall des
} 
nur in Ausnahmefällen nicht gegeben ist, so das es stets einer Prüfung im Einzelfall bedarf, wenn es verneint werden soll ${ }^{32}$.

b) Das Rechtsschutzbedürfnis und der Zweck des Zwischenverfahrens

Gegen die Durchführung eines Zwischenverfahrens unter Beteiligung eines schuldunfähigen Zeugen könnten Bedenken einmal unter dem Gesichtspunkt erhoben werden, der Minderjährigenschutz verbiete die "unnötige" Inanspruchnahme des Zeugen, zum anderen könnte die fehlende Sanktionsmöglichkeit auch im Falle einer Verurteilung dem Rechtsschutzbedürfnis ${ }^{33}$ entgegenstehen.

Das Gebot des Minderjährigenschutzes, dem auch das Prozeßrecht unterliegt, entspricht es, die Verfahrensbeteiligung kindlicher und jugendlicher Zeugen auf das notwendige $\mathrm{Maß}$ zu reduzieren. Dies führt nicht dazu, Minderjährige als ungeeignete Zeugen gänzlich auszuschließen. Es besteht daher auch kein schutzwürdiges Interesse, vor einem Zwischenverfahren geschützt zu werden, weil bis zur rechtskräftigen Entscheidung des Prozeßgerichts nicht zu Lasten des Beweisführers von der Wirksamkeit der Weigerung ausgegangen werden kann.

Ein überwiegendes Schutzinteresse des Minderjährigen kann auch deswegen nicht angenommen werden, weil der Schutz innerhalb des Verfahrens hinreichend gewährleistet werden kann. Hier ist insbesondere auf die Nichtöffentlichkeit der Verhandlung, gegebenfalls unter Beiziehung der Eltern zu verweisen ${ }^{34}$. Der Schutz vor dem Verfahren wäre eine unverhältnismäßige Beschränkung der Rechte des Beweisführers, wenn hinreichender Schutz im Verfahren erreicht werden kann.

Rechtsschutzbedürfnisses; beide Begriffe können daher nicht synonym verwendet werden; dazu Stein-Jonas-Schumann, Vor § 253 Rn 102 Fn 166.

${ }^{32}$ Stephan, S. 28 m.w.N. Letztlich ist dies darauf zurückzuführen, daß die Inanspruchnahme einer gesetzlich vorgesehenen Rechtsschutzmöglichkeit nicht ihrerseits noch einer zusätzlichen Legitimation bedarf.

${ }^{33}$ Das Verfahren nach $§ 387$ ZPO dient lediglich der Feststellung, ob die Zeugnisverweigerung rechtmäßig ist oder nicht. Es ist daher ein Feststellungsinteresse auf Seiten des Beweisführers zu fordern, das sich im Regelfall schon aus dem drohenden Beweismittelverlust ergibt, jedoch durch gegenstehende Zeugenschutzinteressen überwogen sein kann.

${ }^{34}$ Dazu oben $\S 8 \mathrm{~B}, \mathrm{C}$. 
Die rechtskräftige Entscheidung im Zwischenverfahren ist eine Voraussetzung für die Ausübung von Zeugniszwang. Dieser funktionelle Zusammenhang bedeutet aber nicht, daß der Zweck des Zwischenstreits ausschließlich darin gesehen werden könnte, die Sanktionsmöglichkeiten zu eröffnen ${ }^{35}$.

Im übrigen betreffen die Maßnahmen nach $\S 390$ I, II ZPO die zwangsweise Vollstreckung der Zeugenpflichten, während das Zwischenverfahren zunächst nur deren Feststellung bezweckt. Die fehlende Vollstreckungsmöglichkeit ist aber regelmäßig kein Verfahrenshindernis und ohne Auswirkungen auf das Rechtsschutzbedürfnis ${ }^{36}$. Dies gilt selbst dann, wenn mit einer freiwilligen Erfüllung der Zeugenpflichten nicht gerechnet werden $k a_{n}{ }^{37}$. Ein zeitlich nachfolgendes Verhalten des Prozeßgegners, hier des Zeugen oder seiner gesetzlichen Vertreter kann nicht zur Zulässigkeitsvoraussetzung erhoben werden ${ }^{38}$. Ebenso müssen Umstände unberücksichtigt bleiben, die - wie die Schuldfähigkeit des Zeugen - erst bei Verhängung oder Vollstreckung von Ordnungs- und Zwangsmitteln geprüft werden. Es ist somit festzuhalten, daß das Zwischenverfahren auch gegen einen Minderjährigen zulässig ist und der - allgemeine - Gedanke des Rechtsschutzbedürfnisses keine Einschränkungen fordert.

\footnotetext{
${ }^{35}$ Das Rechtsschutzbedürfnis ist im Zusammenhang mit dem Prozeßzweck zu sehen und kann entfallen, wenn der Zweck nicht mehr erreicht werden kann. Vgl. dazu Schönke, S. 24 ff, 31 ff; Stein-Jonas-Schumann, Vor § 253 Rn 117; Thomas-Putzo, Vor $\S 253$ Anm. III A 1 m; Wieser, S. $135 \mathrm{ff}$.

${ }^{36}$ Schönke, S. 37 f; Stein-Jonas-Schumann, Vor § 253 Rn 117; Stephan, S. 52 f.

${ }^{37}$ Dies wird vorwiegend dann der Fall sein, wenn das Kind nicht aussagewillig ist. Fehlt es dagegen lediglich an der Zustimmung der Eltern, so kann bei fortdauernder Aussagebereitschaft mit der Vernehmung begonnen werden, wenn die Entscheidung im Zwischenverfahren rechtskräftig geworden ist.
}

${ }^{38} \mathrm{Vgl}$. Baumgärtel, ZZP $67,423,429 \mathrm{f}$, der darauf hinweist, daß nur prozessuale Gründe für das Fehlen des Rechtsschutzbedürfnisses maßgeblich sein können. 


\section{B. Das Rechtsmittel der Beschwerde}

\section{Gegenstand der Beschwerde durch einen Zeugen}

Die Beschwerde kann sich zum einen nach § 380 III, § 390 III ZPO gegen die Verhängung von Ordnungs- und Zwangsmitteln richten, wenn der Zeuge der Erscheinens- oder Aussagepflicht nicht ordnungsgemäß nachgekommen ist. Zum anderen ist eine Beschwerdemöglichkeit gegen eine Zwischenentscheidung des Prozeßgerichts gemäß § 387 III ZPO vorgesehen, wenn dem Zeugen ein Zeugnisverweigerungsrecht abgesprochen wurde. Letztere ist als sofortige Beschwerde innerhalb einer zweiwöchigen Frist zulässig, $\S 577 \mathrm{ZPO}$.

Gegenstand der Beschwerde ist jeweils eine gerichtliche Entscheidung. Beschwerdegegner ist jedoch nicht das Gericht, sondern stets der Beweisführer ${ }^{39}$. Die Beschwerde nach $\$ 380 \mathrm{III}, \S 390 \mathrm{III} \mathrm{ZPO}$ hat gemäß § 572 I ZPO aufschiebende Wirkung. Auf die Durchführung des Beschwerdeverfahrens im Einzelnen soll hier nicht eingegangen werden. Die Darstellung beschränkt sich auf die Einlegung der Beschwerde durch einen Minderjährigen.

\section{Die Einlegung der Beschwerde durch einen minderjährigen Zeugen}

\section{Der schuld- und einsichtsfähige Zeuge}

Erfüllt ein Zeuge seine gesetzlichen Pflichten nicht, so ist die Verhängung von Ordnungs- und Zwangsmitteln zulässig ${ }^{40}$. Voraussetzung ist Schuldfähigkeit und schuldhafte Verletzung der Zeugenpflichten.

Auch wenn kein Zeugnisverweigerungsrecht besteht, ist der Minderjährige in diesen Fällen einem volljährigem Zeugen gleichzustellen. Diese

\footnotetext{
${ }^{39}$ Baumbach-Lauterbach-Albers, Übers. § 567 Anm. 1.

${ }^{40} \mathrm{Vgl}$. oben $\S 4 \mathrm{C}$ II; § $5 \mathrm{C}$ I, II.
} 
Gleichstellung betrifft auch das Recht zur Einlegung einer Beschwerde. Der Minderjährige ist als prozeßfähig anzusehen ${ }^{41}$; wie beim Zwischenstreit entscheidet die alleinige Ausübungsbefugnis und die damit verbundene Rechtsstellung. Diese Rechtsstellung kann auch nicht davon abhängig gemacht werden, ob im Einzelfall ein Zeugnisverweigerungsrecht besteht oder nicht, zumal ein Aussageverweigerungsrecht nach § $384 \mathrm{Nr}$. 1 ZPO im Laufe der Vernehmung entstehen kann. Es genügt daher, wenn der Minderjährige ein Zeugnisverweigerungsrecht selbständig ausüben könnte, um ihn bezüglich aller mit der Zeugenstellung zusammenhängenden prozessualen Handlungen als prozeßfähig anzusehen.

\section{Die Beschwerde des nicht einsichtsfähigen Minderjährigen}

Demgegenüber ist fraglich, ob der noch nicht einsichtsfähige Zeuge gegen die - regelmäßig zu Unrecht ergangenen - Ordnungsmaßnahmen selbst mit dem Rechtsmittel der Beschwerde vorgehen kann, oder ob er hierzu der Mitwirkung der gesetzlichen Vertreter bedarf.

Die fehlende Entscheidungsfähigkeit hat zugleich zur Folge, daß er im Zwischenverfahren nicht prozeßfähig ist ${ }^{42}$. Für das Beschwerderecht ergäbe sich daraus, $d a ß$ es nur den gesetzlichen Vertretern zusteht. Diese könnten allein die Rechte des Kindes kraft ihres Sorgerechts wahren.

Dagegen bestehen jedoch Bedenken, weil der Ausschluß des Beschwerderechts zunächst dessen Rechtsschutzmöglichkeiten verkürzt. Im Regelfall wirkt sich dies nicht aus, weil die gesetzlichen Vertreter die Interessen des Kindes wahrnehmen; zwingend ist dies jedoch nicht, wie die Möglichkeit einer Interessenkollision zeigt. Daher erscheint der Ausschluß der Beschwerdebefugnis des Minderjährigen durch den Gedanken erhöhter Schutzbedürftigkeit und der deswegen eingeräumten elterlichen Sorge für sich allein nicht gerechtfertigt.

Das Recht auch eines nicht Prozeßfähigen zur selbständigen Einlegung von Rechtsmitteln ist in Literatur und Rechtsprechung unter engen Voraussetzungen und mit unterschiedlichen Begründungen anerkannt. So gilt ein

\footnotetext{
${ }^{41}$ Ebenso Bosch, S. 56 unter Berufung auf die Beschwerdebefugnis Minderjähriger nach § 59 FGG. Zum Vergleich mit der Situation im Strafverfahren vgl. unten § 10 B II 2.

${ }^{42}$ Oben $\S 10$ A III 1.
} 
Prozeßunfähiger als prozeßfähig, soweit im Verfahren gerade über die Prozeßfähigkeit gestritten wird ${ }^{43}$. Diese Vorverlagerung der Prozeßfähigkeit ${ }^{44}$ schließt die Einlegung von Rechtsmitteln ein, wenn damit ein erstinstanzliches Urteil gerade mit der Begründung angegriffen wird, es habe wegen der fehlenden Prozeßfähigkeit kein Sachurteil ergehen dürfen ${ }^{45}$. Der Grund für die Abweichung von der gesetzlichen Regelung, die in den genannten Fällen zur Abweisung der Klage und Zurückweisung der Rechtsmittel führen würde, wird im Schutz des Prozeßunfähigen ${ }^{46}$ und in der Gewährung des rechtlichen Gehörs ${ }^{47}$ gesehen. Das Bundesverfassungsgericht sieht darin einen allgemeinen Grundsatz unserer Rechtsordnung ${ }^{48}$, weshalb man davon ausgehen kann, daß die Ausnahmen von der gesetzlich geregelten Prozeßfähigkeit im Sinne der $\S \S 51,52 \mathrm{ZPO}$ auf ähnliche Fallgestaltungen anwendbar sind.

Dafür spricht auch, daß ein Prozeßunfähiger als prozeßfähig angesehen wird, wenn er sich mit Rechtsmitteln gegen die Vollstreckung aus einem gerade wegen der fehlenden Prozeßfähigkeit zu Unrecht ergangenen Titel zur Wehr setzt ${ }^{49}$. Die Beschwerde richtet sich zwar nicht gegen einen Voll-

\footnotetext{
${ }^{43} B G H$, NJW 1957, 989 f; Rosenberg-Schwab, § 44 IV 2; Zöller-Vollkommer, § 50 Rn 8 zum sogenannten Zulassungsstreit. Es handelt sich hier um das Problem der "doppelrelevanten Tatsachen", die sowohl im Rahmen der Zulässigkeits- als auch der Begründetheitsprüfung von Bedeutung sind. Die fehlende Prozeßfähigkeit des Minderjährigen im Sinne der $\S 51 \mathrm{f} \mathrm{ZPO}$ steht zwar fest, nicht aber die Ausübungsbefugnis bezüglich des Zeugnisverweigerungsrechts. Die Ausübungsbefugnis wird daher bei der Zulässigkeit zunächst fingiert; stellt sich bei der Frage der Begründetheit heraus, da $\beta$ der Minderjährige nicht die notwendige Einsichtsfähigkeit besitzt, wird seine Beschwerde als unzulässig zurückgewiesen. Vgl. zur Behandlung der "doppelrelevanten Tatsachen" Stein-Jonas-Schumann, Vor § 355 Rn 32 m.w.N..

${ }^{44}$ Auch nach Wacke, FS der Universität Köln, S. 325, 355 ff handelt es sich um fingierte Prozeßfähigkeit.

${ }^{45}$ BGHZ 18, 184, 190; FamRZ 1972, 35; Baumbach-Lauterbach-Albers, Grundz. § 511 Anm. 2 C; ebenda, § 567 Anm. 2 C; Stein-Jonas-Leipold, § 56 Rn 16; Zöller-Vollkommer, § 56 Rn 13.

${ }^{46}$ Grundmann, S. 46 f zum Minderjährigenschutz; Hager, ZZP 97, 174, 178 ff. Den Schutzgedanken betont im Ergebnis auch Röhl, JZ 1956, 309 ff, der deshalb ein eigenes Beschwerderecht des Entmündigten oder Pfleglings gegen die Entmündigung bzw. Pflegschaft annimmt.

${ }^{47}$ So etwa zum Zulassungsstreit Wacke, FS der Universität Köln, S. 325, 357; Kahlke, ZZP 100, 10, 17 f; Stein-Jonas-Leipold, §56 Rn 16 unter Hinweis auf die Vollständigkeit des Rechtsschutzes.

${ }^{48} B$ VerfGE 10, 302, 306; ebenso $B G H Z 35,1,11 \mathrm{ff}$.

${ }^{49}$ Dazu Hager, ZZP 97, 174, 178 ff.
} 
streckungstitel; die Ordnungsmaßnahme belastet den Betroffenen aber nicht minder, weil er der Gefahr der Beitreibung ausgesetzt ist.

Da etwaige Ordnungs- und Zwangsmittel gerade wegen der fehlenden Schuldfähigkeit unzulässig sind, ist in entsprechender Anwendung dieser Grundsätze auch der Minderjährige selbst beschwerdeberechtigt.

Die eigene Beschwerdebefugnis ist auch nicht deshalb abzulehnen, weil sie sich zum Nachteil des Minderjährigen auswirken könnte, wenn dieser zu einer sachgerechten Wahrnehmung seiner Rechte im Beschwerdeverfahren nicht in der Lage ist. Zum einen ist das Prozeßgericht oder das Beschwerdegericht schon von Amts wegen verpflichtet, die Sanktionsfolge aufzuheben, ohne daß es umfangreicher Beweiserhebungen bedarf. Die fehlende Schuldfähigkeit beruht im Regelfall darauf, daß der Zeuge das 14. Lebensjahr noch nicht vollendet hat, was ohne weiteres feststellbar ist. Zum anderen schließt die Beschwerdebefugnis des kindlichen Zeugen die Beschwerde durch die gesetzlichen Vertreter nicht aus ${ }^{50}$.

\section{Beschwerderecht der gesetzlichen Vertreter}

Nach § 298 I StPO kann der gesetzliche Vertreter eines Beschuldigten selbständig Rechtsmittel zugunsten des Verurteilten einlegen. Der gesetzliche Vertreter handelt dabei nicht im Namen des Beschuldigten; er übt vielmehr ein eigenes Recht aus ${ }^{51}$. Die gleichzeitige Einlegung desselben Rechtsmittels durch den Beschuldigten wird dadurch nicht ausgeschlossen ${ }^{52}$; sie hat lediglich zur Folge, daß das Rechtsmittelgericht zur Prüfung aller vorgebrachten Beschwerdegründe verpflichtet ist. Das Strafprozeßrecht anerkennt somit eine eigene Beschwerdebefugnis auch des minderjährigen Beschuldigten, ohne diese von zusätzlichen Voraussetzungen abhängig zu machen. Für den Zeugen fehlt auch in der Strafprozeßordnung eine entsprechende Regelung ${ }^{53}$. Eine abweichende Behandlung der Rechtsbehelfsbefugnis ist aber nicht gerechtfertigt, weil bei der Beschwerde des minder-

\footnotetext{
${ }^{50} \mathrm{Im}$ Ergebnis ähnlich Fehnemann, S. 48 f zum Verwaltungsverfahren.

${ }^{51}$ Kleinknecht/Meyer, § $298 \mathrm{Rn} 1$; Löwe-Rosenberg-Gollwitzer, § $298 \mathrm{Rn} 3$.

${ }^{52}$ Kleinknecht/Meyer, § $298 \mathrm{Rn} 2$; Löwe-Rosenberg-Gollwitzer, § $298 \mathrm{Rn} 4$.

${ }^{53} \S 304$ II StPO betrifft nur die Zeugenbeschwerde als solche, ohne auch für den Minderjährigen eine Regelung zu treffen.
} 
jährigen Zeugen im Zivilprozeß die relevanten Umstände von Amts wegen zu prüfen sind. Die Prüfungspflicht des Zivilgerichts unterscheidet sich daher insoweit nicht von der des Strafrichters.

Können daher sowohl der Minderjährige als auch die gesetzlichen Vertreter Beschwerde einlegen, kommt es auf die Gefahr widersprüchlicher Prozeßhandlungen nicht an. Das Prozeßgericht ist schon von sich aus zur Aufhebung von unzulässigen Ordnungs- und Zwangsmitteln verpflichtet und hat daher alles tatsächliche Vorbringen zu berucksichtigen.

\section{Ergebnis}

Im Zwischenverfahren zur Feststellung der Rechtmäßigkeit der Zeugnisverweigerung ist der Minderjährige selbst prozeßfähig, wenn er die Verstandesreife besitzt, über den Verzicht auf das Weigerungsrecht wirksam entscheiden zu können. Fehlt dem Zeugen diese Fähigkeit, so muß er in dem Verfahren nach $\S 387$ ZPO von den Eltern oder einem Ergänzungspfleger vertreten werden, weil er nicht prozeßfähig im Sinne der $\S \S 51,52 \mathrm{ZPO}$ ist.

Soweit dem Zeugen ein Beschwerderecht zusteht, kann sie unabhängig von der Verstandesreife und Einsichtsfähigkeit von dem Minderjährigen selbst eingelegt werden. Bei fehlender Reife können daneben die Inhaber des Personensorgerechts die Beschwerde für den kindlichen Zeugen einlegen. 
Dritter Abschnitt

\section{Das Verfahren vor dem Vormundschaftsgericht bei Verhinderung der gesetzlichen Vertreter}

\section{§ 11 Die Entscheidung des Vormundschaftsgerichts}

\section{A. Allgemeines}

\section{Zuständigkeit und Verfahren}

Die Anrufung des Vormundschaftsgerichtes während des laufenden Zivilrechtsstreites dient der Bestellung eines Ergänzungspflegers für den noch minderjährigen Zeugen. Dabei wird in den folgenden Ausführungen im wesentlichen auf die Ausübung eines Zeugnisverweigerungsrechts des minderjährigen Zeugen abgestellt, weil dies den wichtigsten Bereich der prozessualen Befugnisse des Zeugen bildet.

Das Verfahren richtet sich gemäß § 1915 I BGB nach den für die Vormundschaft geltenden Vorschriften des Bürgerlichen Rechts und des Gesetzes über die Angelegenheiten der freiwilligen Gerichtsbarkeit ${ }^{1}$. Sachlich zuständig ist das Vormundschaftsgericht ${ }^{2}$. Die funktionelle Zuständigkeit liegt bei dem Rechtspfleger, da die Entscheidung gemäß $\S 3$ Nr. 2 a i.V.m. $\S 14$ Nr. 4 RPflG nicht dem Richter vorbehalten wurde ${ }^{3}$.

\footnotetext{
${ }^{1}$ MünchKomm-Goerke, § 1915 Rn 5; Soergel-Danrau, § 1909 Rn 19.

${ }^{2}$ Keidel/Kuntze, § 37 Rn 1.

${ }^{3}$ Nachfolgend wird unabhängig von der funktionellen Zuständigkeit vom Vormundschaftsgericht gesprochen.
} 
Das elterliche Sorgerecht wird durch die Bestellung des Pflegers nicht berührt, wenn die gesetzlichen Vertreter infolge eigener Beteiligung am Zivilproze $\beta$ kraft Gesetzes verhindert sind ${ }^{4}$. Die Beteiligung der Eltern und die dadurch bedingte Interessenkollision ersetzt nicht die Notwendigkeit, daß die Pflegschaft ein Fürsorgebedürfnis voraussetzt ${ }^{5}$. Das Fürsorgebedürfnis soll staatliche Eingriffe in das elterliche Sorgerecht ohne rechtlichen Grund verhindern; die Notwendigkeit des Eingriffs ist dabei jedoch stets aus der Sicht des Kindes zu beurteilen.

\section{Die Anrufung des Vormundschaftsgerichts}

Das Vormundschaftsgericht ist vom Prozeßgericht über die Notwendigkeit der Bestellung eines Pflegers in Kenntnis zu setzen. Die Mitteilungspflicht ergibt sich aus §50 I FGG, weil die Zeugenvernehmung und damit die Beweisaufnahme nur mit Zustimmung des Ergänzungspflegers ordnungsgemäß fortgesetzt werden kann ${ }^{6}$.

Dagegen kann die Anzeige der Prozeßparteien oder der Eltern, auch sofern identisch, das Vormundschaftsgericht nicht zur Bestellung eines Pflegers veranlassen. Die Vorschrift des § 1909 II BGB ist insoweit restriktiv anzuwenden. Die genannte Regelung soll die frühzeitige Einschaltung des Vormundschaftsgerichts im Interesse des Pflegebedürftigen gewährleisten ${ }^{7}$. Die Anzeigepflicht setzt jedoch voraus, daß eine Pflegschaft erforderlich ist;

${ }^{4}$ Zur Anwendung der $§ 1629$ II 1 , § 1795 I Nr. 3 BGB und der Gegenansicht vgl. oben $\S 6$ C. Nach der Gegenansicht müßte man eine Konstitutivwirkung der vormundschaftsgerichtlichen Entscheidung annehmen. Das Vormundschaftsgericht müßte dann durch gesonderten Beschluß zunächst die elterliche Sorge entziehen, MünchKomm-Goerke, $\S 1909 \mathrm{Rn} 56$. Sind die Eltern nicht aufgrund ihrer Prozeßbeteiligung, sondern nach Maßgabe des § 1666 BGB ausgeschlossen, so ist gemäß $\$ 14 \mathrm{Nr} 8 \mathrm{RPflG}$ der Vormundschaftsrichter zuständig; SoergelDamrau, § 1909 Rn 19.

5Erman-Holzhauer, § 1909 Rn 11; Schaub, FamRZ 1966, 134, 135; Soergel-Damrau, § 1909 Rn 10. Weitergehend OLG Stuttgart, RPfleger 1982, 183, 184; dazu unten Fn 16.

${ }^{6}$ Die Mitteilungspflicht besteht über den Wortlaut des $\S 50$ I FGG hinaus auch dann, wenn die Anordnung der Pflegschaft zur Durchführung des Verfahrens erforderlich ist, Keidel/Kuntze, § $50 \mathrm{Rn} 2$.

${ }^{7}$ MünchKomm-Goerke, § 1909 Rn 5. 
die Erforderlichkeit, die sich aus dem Fürsorgebedürfnis ergibt $^{8}$, kann mit verbindlicher Wirkung nur vom Prozeßgericht, nicht vom Vormundschaftsgericht oder Dritten festgestellt werden.

Ernennt das Vormundschaftsgericht dennoch auf Antrag eines Prozeßbeteiligten für den Zeugen einen Pfleger, so wird den gesetzlichen Vertretern die Zustimmungsbefugnis im Rahmen des dem Pfleger übertragenen Aufgabenbereichs entzogen. Der Beschluß des Vormundschaftsgerichts hat dann konstitutive Wirkung, es sei denn, er wäre aus anderen Gründen nichtig9. Die Pflegerbestellung, deren Rechtmäßigkeit das Prozeßgericht nicht überprüfen darf ${ }^{10}$, hat auf das Zivilverfahren allerdings nur Auswirkungen, wenn auch das Prozeßgericht zu der Überzeugung gelangt, daß ein Fürsorgebedürfnis besteht. Nur dann ist der kindliche Zeuge zu einer selbständigen Ausübung seiner prozessualen Befugnisse nicht in der Lage ${ }^{11}$. In diesem Fall muß das Prozeßgericht aber eine Überprüfung der Entscheidung des Vormundschaftsgerichts herbeiführen. Die Fürsorgepflicht gegenüber dem Zeugen verbietet es, von der Wirksamkeit der Pflegerbestellung ungeachtet ihrer Rechtmäßigkeit auszugehen ${ }^{12}$.

\footnotetext{
${ }^{8}$ Dazu sogleich unter $\S 11 \mathrm{~B}$.
}

${ }^{9}$ Die (nur) rechtswidrige Pflegschaftsanordnung ist aufhebbar, aber wirksam, $B G H Z$ 41, 303, $309=$ NJW 1964, 1855 f; BGH bei Holtz, MDR 1987, 448.

${ }^{10}$ Soergel-Damrau, § 1909 Rn 20; § 1915 Rn 5 a.E..

${ }^{11}$ Sieht das Prozeßgericht trotz der - rechtswidrigen - Pflegerbestellung wegen der Zustimmungsverweigerung der Eltern von der Vernehmung ab, so kann darin eine Verletzung des rechtlichen Gehörs des Beweisführers liegen.

${ }^{12} B G H Z$ 41, 303, $309 \mathrm{f}=$ NJW 1964, 1855 f zur Bestellung eines Prozeßpflegers für eine Partei. Die grundlegenden Erwägungen der Entscheidung treffen gleichermaßen für den Zeugen zu, denn der $B G H$ begründet sein Urteil mit dem tiefen Eingriff in das Persönlichkeitsrecht des Betroffenen, den die Anordnung einer Pflegschaft darstellt. 


\section{Auswirkungen auf den Zivilprozeß}

Für die Dauer des vormundschaftsgerichtlichen Verfahrens kann der Zivilprozeß fortgesetzt werden, soweit die Beweisaufnahme nicht gerade die Vernehmung des jugendlichen Zeugen erfordert. Die Vernehmung eines minderjährigen Zeugen sollte jedoch stets erst angeordnet werden, wenn alle anderen zugänglichen Beweismittel erschöpft wurden, ohne zu einer Überzeugungsbildung des Gerichts zu führen ${ }^{13}$. In diesem Fall ist die Vernehmung des Minderjährigen entscheidend für die Fortführung des Verfahrens.

Da die Vernehmung zur Sache erst nach der wirksamen Zustimmung des Pflegers erfolgen darf, muß die Entscheidung des Vormundschaftsgerichts abgewartet werden. Das Prozeßgericht hat die Möglichkeit, das Verfahren in entsprechender Anwendung des $\S 148$ ZPO auszusetzen ${ }^{14}$. Dagegen haben die Parteien die Möglichkeit der Beschwerde gemäß § $252 \mathrm{ZPO}^{15}$.

\section{B. Fürsorgebedürfnis als Voraussetzung der Pflegschaftsanordnung}

\section{Notwendigkeit der Pflegerbestellung}

\section{Fehlende Verstandesreife}

Ein Ergänzungspfleger ist immer nur dann zu bestellen, wenn der Minderjährige die prozessualen Befugnisse eines Zeugen nicht eigenständig wahrnehmen kann und die Eltern an der Ausübung des Sorgerechts gehindert sind ${ }^{16}$. Der Hauptanwendungsfall betrifft die Ausübung eines Zeugnis-

\footnotetext{
${ }^{13}$ Dazu oben $\S 3$ B III 2 c.

${ }^{14} \mathrm{Zur}$ analogen Anwendung des $\S 148 \mathrm{ZPO} B G H Z 41,303,310=$ NJW 1964, $1855 \mathrm{f}$; Thomas-Putzo, § 148 Anm. 3; Zöller-Stephan, § 148 Rn 10.

${ }^{15}$ Zur Beschwerdemöglichkeit wegen faktischem Verfahrensstillstand durch den Beweisbeschluß, den minderjährigen Zeugen zu vernehmen, unten $§ 11 \mathrm{E} \mathrm{I}$.

${ }^{16} \mathrm{Zu}$ weitgehend $O L G$ Stuttgart, RPfleger 1982, 183 f, wonach das Fürsorgebedürfnis allein
} 
verweigerungsrechts, daneben können auch Entscheidungen über die Mitwirkung an Glaubwürdigkeits- und Eignungsgutachten ${ }^{17}$, die Einlegung von Rechtsbehelfen ${ }^{18}$ oder im Zusammenhang mit einem Zwischenverfahren ${ }^{19}$ notwendig werden.

Das Prozeßgericht muß vor Beginn der Vernehmung zur Sache feststellen, ob der Minderjährige zur selbständigen Rechtsausübung in der Lage ist ${ }^{20}$. Eine Vernehmung in der Hoffnung, in ihrem Verlauf werde sich die Verstandesreife und damit die Wirksamkeit des Verzichts auf das Weigerungsrecht herausstellen, genügt nicht. Schon aufgrund seiner Fürsorgepflicht ${ }^{21}$ ist das Gericht gehalten, durch gezielte Fragen die Entscheidungsfähigkeit und Reife des minderjährigen Zeugen für jede Befugnis festzustellen. Es darf nicht davon ausgehen, da $\beta$ aufgrund des Alters eine Vermutung für oder gegen die Reife der Beweisperson spreche ${ }^{22}$.

\section{Aussagebereitschaft des minderjährigen Zeugen}

Das Fürsorgebedürfnis setzt neben der erwähnten Verstandesunreife voraus, da $\beta$ der kindliche Zeuge von sich aus aussagebereit is $t^{23}$. Er ist bei fehlender Reife zwar nicht zum rechtswirksamen Verzicht auf das Zeugnisverweigerungsrecht in der Lage; umgekehrt wird das Kind aber weder durch die Zustimmung der Eltern noch der des Pflegers zur Aussage verpflichtet.

schon dadurch begründet wird, daß über ein Recht des unreifen Kindes entschieden werden müsse. Die Entscheidung berücksichtigt nicht, daß auch der Unreife über die Ausübung des Zeugnisverweigerungsrechts entscheiden kann und nur der Verzicht auf das Recht der Zustimmung Dritter bedarf.

${ }^{17}$ Dazu oben § 3 B IV.

${ }^{18}$ Siehe bei $\S 10 \mathrm{~B}$.

${ }^{19} \mathrm{Vgl}$. oben $\$ 10 \mathrm{~A}$ III.

${ }^{20}$ BayObLG, NJW 1967, 206, 209 = FamRZ 1966, 644, 647; LG Mannheim, MDR 1971, 663 = FamRZ 1974, 158; OLG Hamm, OLGZ 1972, 157, 158; OLG Stuttgart, FamRZ 1985, 1154, $1155=$ OLGZ 1985, 385, 387 zum Strafverfahren. MünchKomm-Hinz, §1626 Rn 50; Schneider, JurBüro 1970, 1021, 1022; Soergel-Damrau, § 1795 Rn 8.

${ }^{21}$ Zur Fürsorgepflicht s.o. $\$ 8 \mathrm{~A}$.

${ }^{22}$ MünchKomm-Goerke, $\S 1909 \mathrm{Rn} 50$ verweist darauf, daß die Ergänzungspflegschaft nicht ohne weiteres, daß heißt ohne vorherige Feststellung der Verstandesreife angeordnet werden dürfe. Dazu auch unten $\S 11$ A II.

${ }^{23}$ LG Memmingen, MDR 1982, 145; OLG Stuttgart, FamRZ 1985, 1154, 1155 = OLGZ 1985, 385, 387 f; MünchKomm-Hinz, § 1626 Rn 50. 
Daraus ergibt sich, daß bei fehlender Aussagewilligkeit auch bei Zustimmung des Pflegers keine Gewähr besteht, daß es zu einer Aussage kommt. Bei anfänglicher und fortdauernder Aussageverweigerung bedarf es daher keiner Entscheidung des Vormundschaftsgerichts.

Einschränkend ist die Zulässigkeit der Pflegerbestellung zu bejahen, wenn die Aussagebereitschaft erst im Verlauf des vormundschaftsgerichtlichen Verfahrens entfällt. Zwar fehlt dann zugleich das Fürsorgebedürfnis, doch ist die Bestellung des Pflegers aus Zweckmäßigkeitsgründen vorzunehmen. Nur so kann eine weitere Verzögerung des Zivilverfahrens vermieden werden, wenn es zu einem weiteren Sinneswandel des Kindes kommt. Dies gilt umso mehr, wenn die Anhörung des Betroffenen gemäß $\S 50 \mathrm{~b}$ FGG bereits stattgefunden hat und die Sache vor dem Vormundschaftsgericht ohne weitere Belastung des Kindes entscheidungsreif ist.

\section{Rücknahme des Beweisantrages}

Bei Zurücknahme des Beweisantrages durch die beweisführende Partei besteht für die Entscheidung des Vormundschaftsgerichts kein Bedürfnis mehr, es tritt Erledigung des Verfahrens ein ${ }^{24}$. Eines Antrages einer Partei oder des Prozeßgerichtes bedarf es nicht; das Vormundschaftsgericht stellt die Erledigung lediglich von Amts wegen formlos fest ${ }^{25}$. Dasselbe gilt, wenn das Prozeßgericht den Beweisantrag wegen fehlender oder nicht nachgewiesener Zeugeneignung verwirf $\mathrm{f}^{26}$. Auch dann fehlt für die Entscheidung des Vormundschaftsgerichts das Interesse, weil es zu einer Vernehmung als Zeuge nicht mehr kommen wird.

\footnotetext{
${ }^{24}$ Zur Erledigung im FGG-Verfahren allgemein Keidel/Kohl, § $19 \mathrm{Rn} 88$.

${ }^{25} \mathrm{Keidel} / \mathrm{Kohl}$, § $19 \mathrm{Rn} 91$. Die Kosten des vormundschaftsgerichtlichen Verfahrens können nach Maßgabe des §13a FGG den Prozeßparteien auferlegt werden; dazu Keidel/Zimmermann, §13a Rn 44.

${ }^{26} \mathrm{Vgl}$. 3 B III.
} 


\section{Die vorsorgliche Bestellung eines Pflegers}

Die Anrufung des Vormundschaftsgerichts während des laufenden Zivilprozesses führt zwangsläufig zu einer Verzögerung des Verfahrens. Um dies zu vermeiden, wird erwogen, bereits vorsorglich einen Pfleger bestellen zu lassen, wenn eindeutig die Verstandesreife fehlt oder die Eltern unzweifelhaft rechtlich verhindert sind ${ }^{27}$.

Dem ist jedoch entgegenzuhalten, daß jede vorsorgliche Pflegschaftsanordnung einen sachlich nicht gerechtfertigten Eingriff in die Rechte der betroffenen Eltern, aber auch des Kindes darstellt, wenn sich nachträglich herausstellt, daß kein Fürsorgebedürfnis bestand ${ }^{28}$. Das Vormundschaftsgericht muß den Minderjährigen vor seiner Entscheidung anhören ${ }^{29}$. Bereits diese Anhörung ist eine Belastung des Kindes, denn durch sie wird es zumindest mittelbar mit dem Rechtsstreit konfrontiert. Darüberhinaus ist zu bedenken, daß für die genannte Altersgrenze keine ausreichenden Erfahrungssätze vorliegen. Es kommt vielmehr auf die Konfliktsituation und die Fähigkeit des Kindes an, mit ihr umzugehen. Auf eine Prüfung im Einzelfall kann daher nicht zugunsten einer Vermutung verzichtet werden.

Zu Beginn des Zivilprozesses und der Beweisaufnahme ist nicht absehbar, ob es zu einer Vernehmung des Kindes kommen wird; als frühester Zeitpunkt der Entscheidung des Vormundschaftsgerichts käme daher der Beweisbeschluß des Prozeßgerichts in Betracht. Aber auch dann ist noch ungeklärt, ob das Kind aussagebereit ist oder ob das Prozeßgericht nicht die Verstandesreife bejaht. Auf dieser Grundlage ist ein Eingriff weder in das Sorgerecht der Eltern noch in das Selbstbestimmungsrecht des Kindes gerechtfertigt, die drohende Prozeßverzögerung zu verhindern. Die Möglich-

\footnotetext{
${ }^{27}$ So diejenigen, die bis zu einem bestimmten Alter die Unreife unterstellen. Vgl. etwa MünchKomm-Hinz, § $1626 \mathrm{Rn} 50$, wonach bei einem sieben- bis zehnjährigen Kind die Unreife unterstellt werden könne. Ähnlich auch LG Mannheim, FamRZ 1974, $158=$ MDR 1971, 663.

${ }^{28}$ OL G Stuttgart, FamRZ 1965, 515, 517 hin; OLG Hamm, OLGZ 1972, 157, 159; OLG Stuttgart, FamRZ 1985, 1154, 1155 = OLGZ 1985, 385, 387. Ferner MünchKomm-Goerke, § 1909 Rn 50 .
}

${ }^{29}$ Zum Verfahren im Einzelnen unten § $11 \mathrm{C}$. 
keit bei Prozeßbeginn, daß es im Laufe des Verfahrens zu einem Fürsorgebedürfnis kommen könnte, genügt somit nicht ${ }^{30,31}$.

\section{Bindung des Vormundschaftsgerichts}

Das Vormundschaftsgericht hat von Amts wegen zu prüfen, ob das Fürsorgebedürfnis besteht ${ }^{32}$. Für die Bestellung eines Ergänzungspflegers zur Wahrnehmung prozessualer Befugnisse des Zeugen muß dieser Grundsatz jedoch eingeschränkt werden.

Das Vorliegen der Aussagebereitschaft ist vom Vormundschaftsgericht nicht zu prüfen, denn selbst bei Aussageunwilligkeit vor dem Vormundschaftsgericht wird - nur für diesen Fall vorsorglich - ein Pfleger bestellt. Es kommt allein darauf an, daß der Minderjährige vor dem Prozeßgericht zur Auskunft bereit war.

Auch eine Überprüfung der Verstandesreife ist dem Vormundschaftsgericht verwehrt ${ }^{33}$. Die Feststellung, daß der Minderjährige keinen wirksamen Verzicht auf ein Zeugnisverweigerungsrecht erklären kann und die Eltern als Sorgerechtsinhaber kraft Gesetzes verhindert sind, ist Teil der Beweisaufnahme. Diese erfolgt ausschließlich vor dem Prozeßgericht ${ }^{34}$, nicht dem Vormundschaftsgericht. Letzteres ist daher an die Feststellungen des Prozeßgerichts gebunden ${ }^{35}$.

Könnte das Vormundschaftsgericht den Antrag auf Bestellung eines Ergänzungspflegers zurückweisen, weil der Zeuge nach seiner Ansicht den

\footnotetext{
${ }^{30}$ Allgemein zur Ablehnung vorsorglich angeordneter Pflegschaften Erman-Holzhauer, § 1909 Rn 11; MünchKomm-Goerke, § 1909 Rn 59; Soergel-Damrau, § 1909 Rn 10.

${ }^{31}$ Verweigert der dennoch bestellte Pfleger die Zustimmung und unterbleibt die Vernehmung deshalb, so ist der Beweisführer in seinem rechtlichen Gehör verletzt.

${ }^{32}$ Keidel/Kuntze, § $37 \mathrm{Rn} 1$.

${ }^{33}$ So zum Strafverfahren OLG Hamm, OLGZ 1972, 157, 158 f; LG Memmingen, MDR 1982, 145; OLG Stuttgart, FamRZ 1985, 1154, 1155 = OLGZ 1985, 385, 386; Kleinknecht/Meyer, $§ 52$ Rn 20; Löwe-Rosenberg-Dahs, §52 Rn 32 a.E.. Für den Zivilprozeß Bosch, S. 53; Gernhuber, § 49 VI 7; Soergel-Strätz, § 1629 Rn 18; Soergel-Damrau, § 1795 Rn 8 a.E..

${ }^{34}$ Darunter ist im vorliegenden Zusammenhang auch der ersuchte und der beauftragte Richter zu verstehen, die auf Veranlassung des Zivilrichters tätig werden.

${ }^{35} \mathrm{Im}$ Ergebnis ebenso MünchKomm-Goerke, § 1909 Rn 50 und die in Fn 33 Genannten.
} 
Gewissenskonflikt bereits versteht und zu lösen im Stande ist ${ }^{36}$, würde es in die Beweisaufnahme eingreifen. Das Prozeßgericht wäre nämlich seinerseits nicht an die Auffassung des Vormundschaftsgerichts gebunden, dürfte aber umgekehrt nicht entgegen seiner Überzeugung den kindlichen Zeugen ohne Einverständnis eines zustimmungsbefugten Dritten vernehmen ${ }^{37}$.

Praktisch würde die verbindliche Prüfung durch das Vormundschaftsgericht auf Bedenken stoßen, weil die konkrete Konfliktsituation, in der sich das Kind befindet, vom Zivilrichter besser beurteilt werden kann. Er kennt den bisherigen Verfahrensablauf und das bis dahin gezeigte Verhalten des Kindes und der Eltern und kann auf dieser Grundlage urteilen, ob der Zeuge von der Bedeutung des Zeugnisverweigerungsrechts eine hinreichende Vorstellung entwickelt hat.

\section{Anhörung der Beteiligten}

\section{Rechtliches Gehör und Anhörung im Verfahren der freiwilligen Gerichtsbarkeit}

Der Anspruch auf rechtliches Gehör besteht in jedem gerichtlichem Verfahren, mithin auch in denen der freiwilligen Gerichtsbarkeit ${ }^{38}$. Dies gilt auch, wenn der Rechtspfleger an Stelle des Gerichts handelt ${ }^{39}$. Von der Gewährung des Gehörs ist die Anhörung der Beteiligten zu unterscheiden, die der Ermittlung der Entscheidungsgrundlagen im Rahmen der Amtsermitt-

\footnotetext{
${ }^{36}$ So nur Schaub, FamRZ 1966, 134, 136, die eine Bindung des Prozeßgerichts an die vormundschaftsgerichtliche Beurteilung des Fürsorgebedürfnisses annimmt.

${ }^{37}$ Dazu Schimansky, FS für Pfeiffer, S. 297, 305 f, der im Zusammenhang mit § 52 II StPO zutreffend darauf hinweist, daß dann im Ergebnis die Entscheidung des Vormundschaftsgerichts an Stelle der der Eltern und des Pflegers tritt, was eine sachliche Prüfung über die Ausübung des Zeugnisverweigerungsrechts gerade ausschließt.

${ }^{38}$ BVerfGE 19, 49, 51 zum Beschwerdeverfahren; BGH, FamRZ 1989, 271; Bumiller/Winkler $\S 12$ Rn 9; Keidel/Amelung, § 12 Rn 106 ff, 110 f; MünchKomm-Hinz, ErgBd., Vor §§ 50a ff, Rn 5, 9.
}

${ }^{39}$ Eickmann, RPfleger 1982, 449, 450. 
lung gemäß $\S 12$ FGG dient ${ }^{40}$. Bei den gesetzlich normierten Anhörungspflichten der $\S 50$ a, b FGG steht das rechtliche Gehör im Vordergrund, weil es um Eingriffe in die Rechte der Betroffenen geht ${ }^{41}$.

\section{Anhörung des Minderjährigen}

\section{Gegenstand und Zweck der Anhörung}

Nach § 50b FGG ${ }^{42}$ hat das Vormundschaftsgericht das Kind anzuhören, wenn die Personen- oder die Vermögenssorge betroffen ist und die Neigungen, Bindungen oder der Wille des Kindes für die Entscheidung von Bedeutung $\operatorname{sind}^{43}$. Dasselbe gilt, wenn die Anhörung zur Feststellung des Sachverhaltes notwendig ist. Nach Vollendung des vierzehnten Lebensjahres ist das Kind stets persönlich anzuhören ${ }^{44}$.

Die Entscheidung des Vormundschaftsgerichts muß den Belangen des Kindes Rechnung tragen ${ }^{45}$. Die Bindungen und Interessen des Kindes, die bei der Entscheidung über das Zeugnisverweigerungsrecht bedeutsam sein können, sind auch vom Vormundschaftsgericht zu berücksichtigen, weil die Entscheidung durch die Auswahl eines bestimmten Pflegers beeinflußt werden kann.

\footnotetext{
${ }^{40}$ Zur Abgrenzung Keidel/Amelung, § $12 \mathrm{Rn} 104 \mathrm{f}$.

${ }^{41}$ So Keidel/Amelung, § $12 \mathrm{Rn} 159$ f, der von einem "Recht auf Anhörung" spricht; LG Mannheim, RPfleger 1963, 413 f mit Anmerkung Keidel. Anders aber BayObLG, FamRZ 1980, 1150, 1151 m.w.N., ohne daß es im vorliegenden Zusammenhang auf den Meinungsstreit ankommt.

${ }^{42}$ Eingeführt durch das Gesetz zur Neuregelung des Rechts der elterlichen Sorge vom 18.7.1979, BGBl. I 1061.

${ }^{43}$ Die Entscheidung über ein Zeugnisverweigerungsrecht ist auch in den Fällen des $\S 384 \mathrm{Nr}$. $1 \mathrm{ZPO}$ Teil der Personensorge; dazu $\S 6 \mathrm{~B}$.

${ }^{44}$ In der Literatur ist umstritten, ob die Differenzierung des Gesetzgeber in zwei verschiedene Altersgruppen sachgerecht ist, weil die Erforschung des begründeten Kindeswillens in jedem Fall notwendig sei, eine richtige Entscheidung zu treffen; zum Meinungsstand Moritz, S. $401 \mathrm{ff}$ m.w.N.; de lege lata ist von den unterschiedlichen Anhörungspflichten auszugehen.

${ }^{45}$ BVerfGE 55, 171, 182 = FamRZ 1981, 124, 126 = NJW 1981, 217; Prestien, RdJB 1988, 431, $435 \mathrm{f}$.
} 


\section{Die Durchführung der Anhörung}

Die Anhörung hat mündlich zu erfolgen; bei der Ausgestaltung im übrigen besteht ein weiter Gestaltungsspielraum des Vormundschaftsgerichtes. So kann sie im Sitzungssaal, im Amtszimmer des Rechtspflegers oder auBerhalb des Gerichtsgebäudes in einer dem Kind vertrauten Umgebung durchgeführt werden ${ }^{46}$. Entscheidend ist das Wohl des Kindes, das auch den Ausschluß der Eltern während der Anhörung rechtfertigt ${ }^{47}$.

Da das Kind von zwei Verfahren betroffen ist und bei Beteiligung der Eltern vor allem in familienrechtlichen Streitigkeiten die Gefahr besteht, daß es in den Streit der Eltern hineingezogen wird, liegt eine rasche Anhörung regelmäßig in seinem Interesse.

\section{Absehen von der Anhörung gemäß § 50b III FGG}

Die Anhörung des Kindes kann ausnahmsweise unterbleiben, wenn schwerwiegende Gründe eine nachhaltige und gravierende Beeinträchtigung des Kindes befürchten lassen ${ }^{48}$. Die Interessen der Parteien des Zivilprozesses an einer schnellen Erledigung des Verfahrens scheiden aus.

Stellt bereits die Anhörung im Rahmen des vormundschaftsgerichtlichen Verfahrens eine so ernsthafte Beeinträchtigung des Kindeswohls dar, wird in der Regel auch die Vernehmung des minderjährigen Zeugen vor dem Prozeßgericht eine entsprechende Belastung mit sich bringen. Das Prozeßgericht ist dann zu einer sorgfältigen Prüfung verpflichtet, ob nicht der Beweisantrag wegen fehlender Zeugeneignung zurückzuweisen is ${ }^{49}$.

\footnotetext{
${ }^{46}$ Eschweiler, RdJB 1988, 440, 441; Keidel/Kuntze, §50b Rn 13; Keidel/Amelung, § 12 Rn 166; Moritz, S. 405 f; Prestien, ebenda, S. 436 f; BVerfGE, ebenda (Fn 45).

${ }^{47}$ Keidel/Kuntze, §50b Rn 18; Jansen, §12 Rn 93 ff, 96. Eine Verletzung der Rechte der Eltern liegt darin nicht, weil die Kindesinteressen vorrangig sind und die Eltern zudem ein eigenes Anhörungsrecht nach § 50a FGG haben, Moritz, S. 408 f.

${ }^{48}$ Dazu Keidel/Kuntze, §50b Rn 26 f m.w.N..

${ }^{49} \mathrm{Vgl}$. dazu oben § 3 B III.

11 Findeisen
} 


\section{Anhörung der Eltern}

Neben der Anhörung des Mündels ordnet § 50a FGG auch die der Eltern an. Ziel ist sowohl die Einräumung des rechtlichen Gehörs, als auch Ermittlung der Entscheidungsgrundlagen. Die Anhörung der sorgeberechtigten Eltern ist zwingend ${ }^{50}$. Da die Art und Intensität der Beziehung zwischen Eltern und Kind festgestellt werden soll, ist die persönliche, also mündliche Anhörung vorgeschrieben.

Nicht sorgeberechtigte Eltern oder Elternteile sind nur anzuhören, wenn davon eine Aufklärung zu erwarten ist. Die aufzuklärende Frage ist im vorliegenden Zusammenhang, wer als Pfleger in Betracht kommt. Da das Zeugnisverweigerungsrecht gemäß $\S 383$ I Nr. 3 ZPO durch die verwandschaftliche Beziehung begründet wird und unabhängig vom Sorgerecht besteht, ist die Anhörung auch des nicht sorgeberechtigten Elternteiles regelmäßig sachdienlich. Dies gilt auch dann, wenn der nach § 50a II FGG Anzuhörende nicht Partei des Zivilrechtsstreites ist. Das Weigerungsrecht soll den Zeugen vor einem familiären Konflikt und der damit verbundenen persönlichen Belastung schützen. Um dem Vormundschaftsgericht und anschließend dem Ergänzungspfleger eine sachgerechte Entscheidung zu ermöglichen, ist die Untersuchung der familiären Situation erforderlich. Die Anhörung der Eltern ist dazu wohl in der Regel geeignet.

Die Anhörung sollte daher nur unterbleiben, wenn Gründe des Kindeswohls dies nahelegen. Insoweit kann auf die Ausführungen zur Anhörung des Kindes verwiesen werden ${ }^{51}$.

\footnotetext{
${ }^{50}$ Zwar ist § 50a I FGG als Sollvorschrift formuliert, doch ist dem Vormundschaftsgericht dadurch kein Ermessen eingeräumt, Keidel/Kuntze, § 50a Rn 10.

${ }^{51}$ Oben $\S 11 \mathrm{C}$ II 3. Die $\S 50 \mathrm{a}$ III, § 50b III FGG stimmen inhaltlich überein, Keidel/Kuntze, $\S 50 \mathrm{~b}$ Rn 26.
} 


\section{Die Beteiligung der Prozeßparteien am vormundschaftsgerichtlichen Verfahren}

Am Verfahren zur Bestellung eines Pflegers sind nur die Eltern und das Kind als unmittelbar Betroffene beteiligt. Soweit am Zivilprozeß ein Dritter als Partei teilnimmt, hat er im Verfahren vor dem Vormundschaftsgericht kein Anhörungsrecht ${ }^{52}$. Zum materiell Beteiligten würde der Dritte nur, wenn er durch die zu erwartende oder bereits ergangene Entscheidung des Vormundschaftsgericht unmittelbar in seinen Rechten und Pflichten betroffen würde ${ }^{53}$.

\section{Auswahl des Pflegers durch das Vormundschaftsgericht}

\section{Die Person des Pflegers}

Die Auswahl einer bestimmten Person zum Ergänzungspfleger kann für die zu treffende Entscheidung von erheblicher Bedeutung sein, sie im Einzelfall sogar vorwegnehmen. So kann bei einem Rechtsstreit zwischen den Eltern die Übertragung der Zustimmungsbefugnis auf einen Verwandten der väterlichen oder mütterlichen Linie bereits die Zustimmung bedeuten, wenn die persönlichen Bindungen des Pflegers an die Prozeßparteien eine sachgerechte Abwägung der Kindesinteressen erschweren oder verhindern ${ }^{54}$. Das Vormundschaftsgericht kann daher durch die Auswahl des Pflegers Einfluß ausüben und hat dies bei seiner Entscheidung zu bedenken. Umgekehrt schließt die Beteiligung der Sorgeberechtigten am Zivilverfahren ein elterliches Benennungsrecht gemäß § 1776 BGB aus ${ }^{55}$.

\footnotetext{
${ }^{52} \mathrm{Vgl}$. Eickmann, Rpfleger 1982, 449, 450. Von einer gesetzlichen Regelung der Anhörung Dritter über die Amtsermittlung des § 12 FGG hinaus wurde bewußt abgesehen; dazu MünchKomm-Hinz, ErgBd, Anhang nach § 1693 Vor $\S 50$ a ff $\mathrm{Rn} 3$.

${ }^{53}$ Keidel/Reichert, § 6 Rn 18.

${ }^{54}$ OLG Stuttgart, NJW 1971, 2237, 2240; Erman-Holzhauer, § 1916 Rn 2; Hampel, FamRZ 1964, 125, 128.

${ }^{55}$ Allgemein MünchKomm-Goerke, § 1916 Rn 3.
} 
Die Auswahlentscheidung erfolgt nach Maßgabe des § 1915 I BGB i.V.m. $\S 1779 \mathrm{ff} \mathrm{BGB}^{56}$. Es ist die Person zu wählen, welche dem Wohl des Kindes am besten dienen kann $^{57}$. Die Bestellung des Jugendamtes ist gemäß $\S 1791$ b I BGB nur zulässig, wenn eine geeignete Einzelperson nicht zur Verfügung steht ${ }^{58}$. Für die Entscheidung über die Wahrnehmung prozessualer Befugnisse des Kindes gilt dies ebenfalls, so daß vorrangig Angehörige und Verwandte in Betracht kommen ${ }^{59}$. Dabei ist jedoch besonders darauf zu achten, daß der Pfleger zu einer unbefangenen und am Kindeswohl orientierten Entscheidung fähig ist, die mit den Interessen eines oder beider Elternteile kollidieren $\mathrm{kann}^{60}$.

Bestehen daran Zweifel oder würde die Ermittlung einer geeigneten Person aus dem Verwandschaftskreis unverhältnismäßig viel Zeit in Anspruch nehmen, so ist einer Bestellung des Jugendamtes der Vorzug zu geben ${ }^{61}$. Dies darf jedoch nur im Interesse des Kindes erfolgen. So kann das Kindeswohl gebieten, das Zivilverfahren nicht weiter zu verzögern, weil es sonst für längere Zeit im ungewissen ist, ob es noch zu einer Aussage kommen wird. Auch besteht die Möglichkeit, daß das Kind außerprozessual stärker in den Konflikt der Eltern hineingezogen wird.

\footnotetext{
${ }^{56} \S 1916$ BGB steht nicht entgegen, weil diese Vorschrift nur die Berufung, nicht die Auswahl des Pflegers betrifft, OLG Stuttgart, RPfleger 1982, 183, 184. Ebenso Erman-Holzhauer, $\S 1916 \mathrm{Rn} 2$; MünchKomm-Goerke, § $1916 \mathrm{Rn} 5$, wonach die §§ $1779 \mathrm{ff}$ BGB zumindest ana$\log$ anwendbar sind.

${ }^{57}$ Wesche, RPfleger 1988, 453, 455; OLG Braunschweig, RPfleger 1989, 237, 238.

${ }^{58}$ Wesche, ebenda (Fn 57) hält die Sachbearbeiter des Jugendamtes in der Regel nicht zu einer Betreuung in der Lage und daher für weniger geeignet.

${ }^{59}$ OLG Stuttgart, RPfleger 1982, 183, 184; FamRZ 1985, 1154, 1155 = OLGZ 1985, 385, 386; MünchKomm-Schwab, § $1779 \mathrm{Rn} 6$. Anders dagegen Erman-Holzhauer, § $1915 \mathrm{Rn} 2$; Schoene, NJW 1972, 930, 932, nach denen § 1779 II 3 BGB hinter Abs. II Satz 1 zurücktritt. Kritisch auch $O L G$ Braunschweig, RPfleger 1989, 237 f, das darauf hinweist, daß weder Verwandschaft noch Schwägerschaft etwas über die Eignung zum Vormund aussagen.

${ }^{60}$ MünchKomm-Goerke, $\S 1915$ Rn 4. Prestien, RdJB 1988, 431, 438 empfiehlt bei Familiensachen die Hinzuziehung eines Familienberaters als "quasi Sachverständigen" und die Bestellung eines Juristen als "Verfahrenspfleger".

${ }^{61}$ Ähnlich MünchKomm-Goerke, § 1915 Rn 16. Die Verzögerung kann auch auf den weitergehenden Anhörungspflichten bei der Bestellung eines Verwandten oder Verschwägerten beruhen, § 1779 III BGB.
} 


\section{Die Bestellung des Pflegers}

Mit der Bestellung wird die Ausübung einzelner, vom Vormundschaftsgericht zu bestimmender Angelegenheiten der Personensorge auf den Ergänzungspfleger übertragen. Ein besonderes Verfahren zur Bestellung sehen die § $1915 \mathrm{I}$, § 1789 BGB nicht vor ${ }^{62}$.

Die Bestellung erfolgt durch einen "mitwirkungsbedürftigen Hoheitsakt"63. Sie wird nach § 16 I FGG mit Bekanntgabe wirksam. Sofern es sich um eine natürliche Person handelt, ist ihre persönliche Anwesenheit und die Erklärung erforderlich, das Amt treu und gewissenhaft führen zu wollen. Im Falle der Bestellung des Jugendamtes genügt gemäß § 1791 b II BGB eine schriftliche Verfügung ${ }^{64}$.

\section{E. Rechtsbehelfe}

Die Anrufung des Vormundschaftsgerichts greift in die Rechte und Interessen der Betroffenen ein. So werden die Eltern in ihrem Personensorgerecht, das Kind in seinem Selbstbestimmungsrecht und die Prozeßparteien in ihrem Interesse an einem zügigen Fortgang des Verfahrens berührt. Die Parteien können darüberhinaus mittelbar durch die Entscheidung des Vormundschaftsgerichts oder des Ergänzungspflegers in ihrem Recht auf Beweis betroffen werden. Es soll daher auf die möglichen Rechtsschutzmöglichkeiten eingegangen werden.

\footnotetext{
${ }^{62} \S 1789$ Satz 2 BGB ist eine bloße Ordnungsvorschrift, deren Nichteinhaltung auf die Wirksamkeit der Bestellung keinen Einfluß hat; MünchKomm-Schwab, § 1789 Rn 2.

${ }^{63}$ Erman-Holzhauer, § 1789 Rn 1; MünchKomm-Schwab, ebenda (Fn 62), Rn 1.

${ }^{64}$ Für die Vereinsvormundschaft gilt nach $\$ 1791$ a II BGB dasselbe, doch dürfte die Vorschrift für die Bestellung eines Pflegers für einen minderjährigen Zeugen keine praktische Bedeutung haben.
} 


\section{Beschwerde gegen die Anrufung des Vormundschaftsgerichts}

Die mit der Einschaltung des Vormundschaftsgerichts zwangsläufige Verzögerung des Zivilverfahrens kann von den Parteien entsprechend dem Rechtsgedanken des § 252 ZPO mit der einfachen Beschwerde angegriffen werden $^{65}$. Sie ist jedoch nur begründet, wenn das Prozeßgericht die Notwendigkeit der Pflegerbestellung offensichtiich verkennt; gerade bei der Beurteilung der Verstandesreife besteht ein Spielraum des Zivilrichters, der für das Beschwerdegericht nicht uneingeschränkt überprüfbar ist.

\section{Rechtsschutz gegen die Entscheidung des Vormundschaftsgerichts}

\section{Erinnerungsbefugnis bei abgelehnter Pflegerbestellung}

Lehnt der Rechtspfleger die Bestellung eines Ergänzungspflegers ab, so ist dagegen die Rechtspflegererinnerung des § 11 RPflG statthaft. Erinnerungsbefugt ist jeder, der an der Anordnung der Pflegschaft ein rechtliches Interesse hat; §57 I Nr. 3 FGG ist gemäß § 11 IV RPflG entsprechend anwendbar ${ }^{6}$. Beschwert ist durch die Ablehnungsentscheidung etwa der Beweisführer, dem der Verlust des Beweismittels droht.

Problematisch bleibt die Frage, ob auch dem Prozeßgericht die Rechtsmittelbefugnis zukommt, wenn das Vormundschaftsgericht in Verkennung seiner eingeschränkten Prüfungskompetenz $z^{67}$ das Fürsorgebedürfnis verneint und deshalb den Antrag zurückweist.

Für einen Zeugen im Zivilverfahren wurde diese Frage bisher offenbar noch nicht erörtert. Bei einer prozeßunfähigen Partei wurde dagegen die

\footnotetext{
${ }^{65}$ Dies ergibt sich aus der praktisch verfahrensaussetzenden Wirkung eines Beschlusses, mit dem das Prozeßgericht die Vernehmung des minderjährigen Zeugen von der Zustimmung eines Pflegers abhängig macht. Dazu Baumbach-Lauterbach-Hartmann, § 359 Anm. 3; SteinJonas-Schumann, § 359 Rn 5 m.w.N.; Zöller-Stephan, § 252 Rn 1.

${ }^{66} J a n s e n, \S 57 \mathrm{Rn} \mathrm{42;} \mathrm{Keidel/Kahl,} \$ 19 \mathrm{Rn} 59$. Zum Begriff des rechtlichen Interesses Keidel/Kuntze, § $57 \mathrm{Rn} 3,15$.

${ }^{67} \mathrm{Vgl}$. oben $\S 11 \mathrm{~B}$ III.
} 
Beschwerdebefugnis des Zivilrichters überwiegend abgelehnt ${ }^{68}$. Dies hat auch zu gelten, wenn der Pfleger nicht für eine Partei, sondern einen Zeugen bestellt werden soll. Das Prozeßgericht verfolgt keine eigenen Interessen mit der Pflegschaftsanordnung ${ }^{\theta}$. Es ist an die Feststellungen des Vormundschaftsgerichts bezüglich des Fürsorgebedürfnisses nicht gebunden. Die Vernehmung des nach Meinung des Prozeßgerichts zustimmungsbedürftigen Zeugen kann daher auch nicht unter Berufung auf die Entscheidung des Vormundschaftsgerichts erfolgen. Mangels wirksamen Verzichts auf das Zeugnisverweigerungsrecht hat die Vernehmung zu unterbleiben.

Die Bestellung eines Pflegers durch das Prozeßgericht analog § 57 ZPO kommt gleichfalls nicht in Betracht. Zum einen handelt es sich um eine eng auszulegende Ausnahmevorschrift, die dem Beklagtenschutz dient ${ }^{70}$. Zum anderen ist die Umgehung der Zuständigkeit des Vormundschaftsgerichts nur bei Gefahr im Verzug zulässig, was bei der Zeugenvernehmung regelmäßig schon deswegen nicht der Fall ist, weil die Vorschrift der Partei des Zivilprozesses nicht das Risiko nehmen soll, das die Benennung eines kindlichen Zeugen mit sich bringt.

\section{Erinnerungsbefugnis bei angeordneter Pflegschaft}

Die Anordnung der Pflegschaft kann gemäß § 11 RPflG ebenfalls mit der Erinnerung angefochten werden. Erinnerungsbefugt sind nur die Sorgerechtsinhaber und das Kind, sofern es das vierzehnte Lebensjahr vollendet hat, § 59 III FGG ${ }^{71}$. Die Prozeßparteien sind als solche nicht beschwert, da

\footnotetext{
${ }^{68}$ Begründet wird die ablehnende Haltung mit dem fehlenden rechtlichen Interesse des Gerichts, so etwa Jansen, §57 Rn 12; Keidel/Kuntze, §57 Rn 18a. Anders nur Pickel, NJW 1965, 338, 339 für die Sozialgerichtsbarkeit unter Hinweis auf die Besonderheiten dieses Verfahrens, insbesondere § 72 I SGG. Dagegen aber LG München I, FamRZ 1974, 47 ff.

${ }^{69}$ Das Bestreben, den Rechtsstreit erledigen zu können, ist kein rechtliches Interesse im Sinne des §57 I Nr. 3 FGG, Jansen, §57 Rn 12. Dasselbe gilt für die Beschwerdebefugnis nach § 57 I Nr. 9 FGG, die eine besondere persönliche Beziehung zwischen Beschwerdeführer und dem Kind voraussetzt, Jansen, § 57 Rn 27; Keidel/Kuntze, § 57 Rn 37; LG München I, FamRZ 1974, 47, 48. Die rein prozessual begründete Fürsorgepflicht des Prozeßgerichts gegenüber dem Zeugen genügt dafür nicht, da sie auf das Verfahren beschränkt ist.

${ }^{70}$ Kritisch gegenüber einer ausweitenden Analogie Stein-Jonas-Leipold, § 57 Rn 2.

${ }^{71}$ Soergel-Damrau, § 1909 Rn 20; MünchKomm-Goerke, § 1909 Rn 91.
} 
sich lediglich die Person des Zustimmungsbefugten ändert, ohne die $\mathrm{Zu}$ stimmungsbedürftigkeit zu berühren.

Gegen die Auswahl des Pflegers besteht ein Beschwerderecht nur nach Maßgabe des § 11 I, IV RPflG i.V.m. § 57 I Nr. 9 FGG $^{72}$. Zu beachten ist, daß das Vormundschaftsgericht bei der Auswahl einen Ermessensspielraum hat und nur ein Ermessensfehler die Rechtswidrigkeit der Auswahlentscheidung begründet ${ }^{73}$.

Aufschiebende Wirkung kommt der Erinnerung nicht zu, da es an den besonderen Voraussetzungen des § 24 I FGG fehlt. Dies bedeutet, daß mit der wirksamen, wenn auch gegebenenfalls rechtswidrigen Bestellung und der Zustimmung des Pflegers der Zeuge vernommen werden kann. Die nachträgliche Aufhebung der Bestellung durch den Rechtspfleger, den Vormundschaftsrichter oder das Beschwerdegericht hat keine Auswirkungen auf die Verwertbarkeit der Aussage.

\section{Ergebnis}

Das Prozeßgericht stellt mit verbindlicher Wirkung fest, ob ein Fürsorgebedürfnis besteht und ein Ergänzungspfleger für den Minderjährigen bestellt werden muß. Ein Fürsorgebedürfnis besteht, wenn eine prozessuale Befugnis nicht vom Zeugen allein ausgeübt werden kann und die Sorgeberechtigten rechtlich verhindert sind. Beim Zeugnisverweigerungsrecht muß daneben Aussagebereitschaft des Kindes vorliegen.

Das Vormundschaftsgericht trifft durch den Rechtspfleger die Entscheidung über die Auswahl eines geeigneten Pflegers. Die vorsorgliche Bestellung eines Pflegers ist unzulässig.

Die Parteien haben gegen die Anrufung des Vormundschaftsgerichts die Möglichkeit der einfachen Beschwerde analog § $252 \mathrm{ZPO}$, wenn das Prozeßgericht das Verfahren entsprechend § 148 ZPO aussetzt oder dem Beschluß, einen Pfleger bestellen zu lassen, praktisch verfahrensaussetzende Wirkung zukommt.

\footnotetext{
${ }^{72}$ Soergel-Damrau, § 1909 Rn 20; Soergel-Strätz, § 1630 Rn 3.

${ }^{73}$ MünchKomm-Schwab, § $1779 \mathrm{Rn} 4$; dazu auch oben § 11 D I.
} 
Das Prozeßgericht ist an eine ablehnende Entscheidung des Vormundschaftsgerichts nicht gebunden und hat keine Erinnerungsbefugnis. Lehnt das Prozeßgericht deshalb die Vernehmung des Zeugen ab, können die Parteien gemäß § 11 IV RPflG, § 57 I Nr. 3 FGG Erinnerung einlegen.

Die Auswahl des Pflegers kann nur von den Sorgeberechtigten und gemäß § 59 III FGG von dem Kind angegriffen werden. 



\title{
§ 12 Die Rechtsstellung des Ergänzungspflegers
}

\author{
A. Entscheidungsbefugnisse
}

\section{Allgemeines}

Das Vormundschaftsgericht überträgt mit der Bestellung die Erledigung bestimmter personensorgerechtlicher Angelegenheiten auf den Pfleger. Zugleich wird das Sorgerecht der gesetzlichen Vertreter insoweit gemäß $\S 1630$ I BGB eingeschränkt.

Die praktisch wichtigste Angelegenheit im vorliegenden Zusammenhang ist die Befugnis, der Vernehmung des Kindes zuzustimmen oder von einem Weigerungsrecht Gebrauch zu machen. Das Vormundschaftsgericht sollte den Kreis der übertragenen Aufgaben aber nicht auf diese Entscheidung beschränken ${ }^{1}$, um Verzögerungen des Verfahrens zu vermeiden. Fallen vor dem Prozeßgericht weitere Entscheidungen an, die der Zustimmung des Pflegers bedürfen, müßte sonst das Vormundschaftsgericht erneut angerufen werden². Ähnlich der Pflegschaft zur Führung eines bestimmten Rechtsstreits ist dem Ergänzungspfleger die Wahrnehmung aller prozessualen Befugnisse des minderjährigen Zeugen in einem genau zu bezeichnenden Rechtsstreit zu übertragen.

\footnotetext{
${ }^{1}$ So aber offenbar in dem vom BayObLG, NJW 1967, 206, $207=$ FamRZ 1966, 644 ff entschiedenen Fall.

${ }^{2}$ Nach Roestel, NJW 1967, 967, 968; derselbe, RdJB 1971, 218, 220 ist mit der Entziehung der Zustimmungsbefugnis zum Verzicht auf das Zeugnisverweigerungsrecht nicht zugleich über die Zustimmung zur psychologischen Begutachtung entschieden. Danach müßte das Vormundschaftsgericht darüber neu befinden und gegebenfalls wieder einen Pfleger bestellen.
} 


\section{II. Übertragener Wirkungskreis}

Der Ergänzungspfleger wirkt mit, wenn der minderjährige Zeuge ein Recht nicht oder nicht selbständig ausüben kann. Darunter fallen neben dem Verzicht auf ein Zeugnisverweigerungsrecht die Entscheidung über die Mitwirkung an einer psychologischen Glaubwürdigkeits- und Eignungsbegutachtung ${ }^{3}$. Desweiteren ist der Pfleger zur Entgegennahme gerichtlicher Erklärungen zuständig, so etwa der Ladung ${ }^{4}$ und der Belehrungs.

Wird das Recht zur Zeugnisverweigerung von den Parteien in Abrede gestellt oder der Minderjährige mit unzulässigen Ordnungs- und Zwangsmitteln belegt, vertritt der Pfleger ihn im Zwischen- und Beschwerdeverfahren, soweit der Zeuge zur selbständigen Rechtswahrung noch nicht in der Lage ist ${ }^{6}$.

\section{B. Wirksamkeit der Entscheidung}

Die Erklärungen des Pflegers haben unmittelbar prozessuale Auswirkungen. Das Prozeßgericht hat sie in gleicher Weise zu berücksichtigen, wie die Entscheidungen rechtlich nicht verhinderter Eltern.

Die nachträgliche Aufhebung der Pflegschaft durch das Vormundschaftsgericht berührt die Rechtshandlungen des Pflegers nicht. Zwar regelt §32 FGG nur die fortbestehende Wirksamkeit rechtsgeschäftlicher Handlungen ${ }^{7}$. Um die Gefahr der nachträglichen Unverwertbarkeit der Zeugenaussage und die damit verbundene Einflußnahme des Vormundschaftsge-

\footnotetext{
${ }^{3}$ Dazu oben § 3 B IV.

${ }^{4}$ Zur Ladung des Minderjährigen siehe § 4 B III 2. Die Mitwirkung des Pflegers bei der Ladung ist notwendig, weil der Pfleger wie die gesetzlichen Vertreter verpflichtet ist, das Kind zum Termin mitzubringen. Es handelt sich aber nicht um eine prozessuale Pflicht, die deshalb auch nicht mit den Zwangsmitteln des Prozeßrechts erzwungen werden kann, §4 C III.

${ }^{5} \mathrm{Vgl}$. 7 A II 2.

${ }^{6}$ Oben $\S 10$ A III 1 und $\S 10$ B II 2, III.

${ }^{7}$ Keidel/Reichert, $\S 32 \mathrm{Rn} 1$.
} 
richts auf das Zivilverfahren zu vermeiden, ist § 32 FGG zumindest analog anzuwenden ${ }^{8}$. Die Aufhebung der Pflegschaft wirkt daher nur ex nunc.

\section{Dauer der Pflegschaft}

Die Pflegschaft endet gemäß § 1918 I BGB mit dem Erreichen der Volljährigkeit des Zeugen und nach § 1919 BGB mit der Aufhebung durch das Vormundschaftsgericht ${ }^{9}$. Letzteres kann etwa der Fall sein, wenn im Rechtsstreit ein Parteiwechsel stattfindet und dadurch die Gefahr einer Interessenkollision entfällt ${ }^{10}$.

Die Pflegschaft endet dagegen nicht mit dem Abschluß der Vernehmung des weigerungsberechtigten Kindes; § 1918 III BGB ist nicht anwendbar. Der Wirkungskreis des Pflegers umfaßt nicht nur eine einzelne Befugnis, sondern einen Kreis von Angelegenheiten ${ }^{11}$. Die Zuständigkeit des Pflegers erschöpft sich daher nicht in der einmaligen Zustimmung zur Vernehmung.

\footnotetext{
${ }^{8}$ Im Ergebnis ebenso BayObLG, NJW 1967, 206, 207 = FamRZ 1966, 644, 645. § 32 FGG soll die Sicherheit des Rechtsverkehrs schützen und drückt daher einen allgemeinen Rechtsgedanken aus, der auf die vorliegende Sachlage ebenfalls anwendbar ist.

${ }^{9}$ Nach Schoene, NJW 1972, 930, 932 vertritt der Pfleger das Kind bis zum Erreichen der notwendigen Verstandesreife. Der Wegfall des Fürsorgebedürfnisses führt jedoch nicht zur Beendigung der Pflegschaft, sondern schafft die Voraussetzungen ihrer Aufhebung; ErmanHolzhauser, § $1919 \mathrm{Rn} 2$. Für den Zivilprozeß ist die Aufhebung in diesem Fall aber bedeutungslos, weil der Pfleger nur Kontrollfunktion hat und der Minderjährige mit Erreichen der Verstandesreife einem volljährigen Zeugen gleichgestellt wird. Er entscheidet dann bereits allein wirksam über den Verzicht auf ein Zeugnisverweigerungsrecht.

${ }^{10}$ Der Wegfall der rechtlichen Verhinderung der gesetzlichen Vertreter ist Aufhebungsgrund, MünchKomm-Goerke, § 1919 Rn 12.

${ }^{11}$ Anders MünchKomm-Goerke, § 1918 Rn 20; Soergel-Damrau, § 1918 Rn 4, die aber als übertragene Angelegenheit ausschließlich die Entscheidung über ein Zeugnisverweigerungsrecht sehen. Wie hier BayObLG, NJW 1967, 206, 207 = FamRZ 1966, 644, 645.
} 


\section{Ergebnis}

Der Ergänzungspfleger tritt an die Stelle der ausgeschlossenen gesetzlichen Vertreter und nimmt deren Kontrollbefugnisse wahr. Die Entscheidungen des Pflegers bleiben auch nach Aufhebung der Pflegschaft wirksam.

Die Pflegschaft endet mit Erreichen der Volljährigkeit und mit ihrer Aufhebung durch das Vormundschaftsgericht. Die Aufhebung erfolgt bei Wegfall des Fürsorgebedürfnisses. 


\section{Zusammenfassung}

Der historische Gesetzgeber hat bewußt darauf verzichtet, bei Kindern und Jugendlichen die Zeugeneignung einzuschränken. Der Grundsatz der freien Beweiswürdigung darf aber nicht zur Mißachtung der natürlichen Grenzen der Wahrnehmungs- und Aussagefähigkeit führen.

Das rechtliche Gehör der Parteien und die Beweiserhebungspflicht des Gerichts erstreckt sich nicht auf ungeeignete Beweismittel. Minderjährige sind vor Erreichen des sechsten Lebensjahres als regelmäßig ungeeignet anzusehen. Sie sind nur zu laden und zu vernehmen, wenn der Beweisführer die Vermutung der fehlenden Eignung widerlegt.

Für die prozessuale Stellung des minderjährigen Zeugen ist die Verstandesreife entscheidend. Auf die Prozeßfähigkeit im Sinne der $§ \S 50 \mathrm{ff}$ ZPO kommt es nur im Zwischen- und Beschwerdeverfahren an, wenn der Minderjährige auf der Passivseite beteiligt ist.

Besitzt der kindliche Zeuge die Fähigkeit, eine Befugnis zu verstehen und eine verantwortliche Entscheidung auszuüben, wird er einem Volljährigen gleichgestellt. Soweit eine eigenverantwortliche Ausübung aufgrund des Alters und der Entwicklung ausgeschlossen ist, sind die gesetzlichen Vertreter hinzuzuziehen. Ihre Mitwirkung beschränkt sich auf die Kontrolle des Kindeswillens, soweit höchstpersönliche Entscheidungen zu treffen sind. Dies gilt insbesondere für die Ausübung eines Zeugnisverweigerungsrechts.

Die gesetzlichen Vertreter sind kraft Gesetzes ausgeschlossen, wenn einer von ihnen selbst als Partei am Verfahren beteiligt ist. Das Prozeßgericht muß bei dem Vormundschaftsgericht die Bestellung eines Ergänzungspflegers veranlassen, wenn eine Vernehmung des Kindes nicht aus anderen Gründen wie fehlender Aussagebereitschaft ausgeschlossen ist. Das vormundschaftsgerichtliche Verfahren richtet sich nach den allgemeinen Vorschriften des FGG. 



\section{Literaturverzeichnis}

Alsberg, Max: Der Beweisantrag im StrafprozeB, begründet von Max Alsberg, fortgeführt von Karl-Heinz Nüse; 5. völlig neu bearbeitete Auflage von Karlheinz Meyer, Köln, Berlin, Bonn, München 1983.

Arntzen, Friedrich: Psychologie der Zeugenaussage, Göttingen 1970.

- Untere Grenze der Zeugeneignung, DRiZ 1976, 20.

- Vernehmungspsychologie - Psychologie der Zeugenaussage, München 1978.

Baumbach, Adolf: Zivilprozeßordnung, fortgeführt von Wolfgang Lauterbach, nunmehr verfaßt von Jan Albers und Peter Hartmann, 49. Auflage, München 1991.

Baumgärtel, Gottfried: "Geheimverfahren" im Zivilprozeß zur Wahrung von Geschäftsgeheimnissen nach Schweizer Vorbild?, in: Festschrift für Walther J. Habscheid zum 65. Geburtstag S. 1 ff, herausgegeben von Walter F. Lindacher, Bielefeld 1989 (zitiert: FS f. Habscheid).

- Beweisrechtliche Studien, in: Festschrift der Rechtswissenschaftlichen Fakultät zur 600-JahrFeier der Universität zu Köln S. 165 ff, Köln, Berlin, Bonn, München 1989 (zititiert: FS der Universität Köln).

- Treu und Glauben im Zivilprozeß, ZZP 86, 353 ff (1973).

- Treu und Glauben, gute Sitten und Schikaneverbot im Erkenntnisverfahren, ZZP 69, 89 ff (1956).

Beitzke, Günther: Familienrecht, 23. Auflage, München 1983.

Bergerfurth, Bruno: Das Ausbleiben des Zeugen im ZivilprozeB, JZ 1971, 84 ff.

Bettermann, Karl-August: Hundert Jahre Zivilprozeßordnung - Das Schicksal einer liberalen Kodifikation, ZZP 77, 365 ff (1978).

Bosch, Friedrich Wilhelm: Anmerkung zu BGH 5 StR 533/58, FamRZ 1959, 158.

- Anmerkung zu BGH VI ZR 266/57, FamRZ 1959, 200.

- Die Pflicht zur Duldung von Untersuchungen gemäß § 372a ZPO 1950, DRiZ 1951, 137 ff.

- Grundsatzfragen des Beweisrechts, Bonn 1963.

- Von der richtigen Grenzziehung zwischen Pflicht und Freiheit, DRiZ 1951, 107 ff.

Brehm, Wolfgang: $\$ 890 \mathrm{ZPO}$ n.F.: Sprachregelung oder sachliche Änderung? NJW 1976, $1730 \mathrm{f}$.

Bruns, Rudolf: Grundsatzfragen des Beweisrechts, ZZP 77, 318 ff (1964).

- Zivilprozeßrecht, 2. Auflage München 1979.

Bull, Hans-Joachim: Von der Bequemlichkeit, einem Zeugen zu glauben, DRiZ 1972, $205 \mathrm{f}$.

Bumiller, Ursula: Freiwillige Gerichtsbarkeit: Gesetz über die Angelegenheiten der freiwilligen Gerichtsbarkeit, erläutert von Ursula Bumiller, Karl Winkler, 4. Auflage, München 1987 (zitiert: Bumiller/Winkler). 
Dehmer, Hans: Probleme des Minderjährigenschutzes im Zivilprozeß, Freiburg im Br. 1977.

Denger, Burkhart: Kinder und Jugendliche als Zeugen im Strafverfahren wegen sexuellen Mißbrauchs in der Familie und deren Umfeld, ZRP 1991, $48 \mathrm{ff}$.

Detterborn, Harry / Fröhlich, Hans / Szewczyk, Hans: Forensische Psychologie, Berlin (Ost) 1984.

Dilcher, Hermann: Die prozessuale Verwendungsbefugnis, AcP 1958, 469 ff (1959).

Dippel, Karlhans: Zur Behandlung von Aussagen kindlicher und jugendlicher Zeugen, in: Festschrift für Herbert Tröndle zum 70. Geburtstag, S. 599 ff, herausgegeben von Hans-Heinrich Jeschek und Theo Vogler, Berlin, New York 1989 (zitiert: FS f. Tröndle).

Döhring, Erich: Die Erforschung des Sachverhaltes im Prozeß, Berlin 1964.

Eickmann, Dieter: Das rechtliche Gehör in Verfahren vor dem Rechtspfleger, RPfleger 1982, $449 \mathrm{ff}$.

Erman, Walter: Handkommentar zum Bürgerlichen Recht, herausgegeben von Harm Peter Westermann unter Mitwirkung von Klaus Küchenhoff, 8. Auflage, Münster 1989 (zitiert: Erman-Bearbeiter).

Eschweiler, Peter: Scheidungskinder vor Gericht, RdJB 1988, 440 ff.

Fehnemann, Ursula: Die Innehabung und Wahrnehmung von Grundrechten im Kindesalter, Berlin 1983.

Flume, Peter: Allgemeiner Teil des Bürgerlichen Rechts, 2. Band: Das Rechtsgeschäft, 2. Auflage, Berlin, Heidelberg, New York 1975.

Franke, Dietrich: Rechtliches Gehör und Präklusion - Zur Rechtsprechung des Bundesverfassungsgerichts, NJW 1986, 3049 ff.

Frohn, Hans Georg: Rechtliches Gehör und richterliche Entscheidung, Berlin 1989.

Gamp, Lothar: Die Ablehnung von rechtswidrig erlangten Beweismitteln im Zivilprozeß, DRiZ 1981, $41 \mathrm{ff}$.

Gaul, Hans Friedhelm: Zur Frage nach dem Zweck des Zivilprozesses, AcP 168, 27 (1968).

Gebhard, Hans-Rainer: Das Zeugnisverweigerungsrecht der Presse im Zivilprozeß, Bad Godesberg 1973.

Gernhuber, Joachim: Elterliche Gewalt heute - Eine grundsätzliche Betrachtung, FamRZ 1962, 89 ff.

- Familienrecht, 3. Auflage, München 1980.

Göppinger, H. / Witter H. (Hrsg): Handbuch der forensischen Psychologie, Berlin, Heidelberg, New York 1972.

Goldschmidt, James: Der Prozeß als Rechtslage - Eine Kritik des prozessualen Denkens, Berlin 1925; Neudruck der Ausgabe Aalen 1962 (zitiert: Der Prozeß).

- Zivilprozeßrecht, 2. Auflage, Berlin 1932 (zitiert: ZPR).

Gottwald, Peter: Zur Wahrung von Geschäftsgeheimnissen im Zivilprozeß, BB 1979, $1780 \mathrm{ff}$.

Grundmann, Martin: Der Minderjährige im Zivilprozeß, Bochum 1980.

Grunsky, Wolfgang: Grundlagen des Verfahrensrechts, 2. Auflage, Bielefeld 1974 (zitiert: Grundlagen). 
- Grundzüge des Zwangsvollstreckungs- und Konkursrechts, 4. Auflage, Tübingen 1987 (zitiert: Grundzüge).

Günter, Hans Helmut: Einführung in das Recht des Zeugenbeweises, JA 1979, $427 \mathrm{ff}$.

Habscheid, Walter: Anmerkung zum Urteil des BGH vom 6.12.1965 - VII ZR 149/63, ZZP 79, 452 ff (1966).

- Das Persönlichkeitsrecht als Schranke der Wahrheitsfindung im Prozeßrecht, in: Gedächtnisschrift Hans Peters S. $840 \mathrm{ff}$, Berlin, Heidelberg, New York 1967 (zitiert: FS f. Peters).

- Das Recht auf Beweis, ZZP 96, 306 ff (1983).

Hagen, Horst: Formzwang und Formzweck im Zivilprozeß, JZ 1972, 505 ff.

Hager, Johannes: Die Rechtsbehelfsbefugnis des Prozeßunfähigen, ZZP 97, 174 ff (1984).

Hahn, Carl: Die gesamten Materialien zur Civilprozeßordnung und dem Einführungsgesetz zu derselben vom 30. Januar 1877, Berlin 1880 (zitiert: Materialien).

Hampel, Herbert: Die Weigerungsrechte der minderjährigen Beweisperson im Strafprozeß, FamRZ 1974, 53 ff.

- Grundsatzfragen des Beweisrechts, FamRZ 1964, 125 ff.

Hanack, Ernst-Walter: Die Rechtsprechung des Bundesgerichtshofes zum Strafverfahrensrecht, JZ 1971, 126 ff.

Hauser, Robert: Der Zeugenbeweis im Strafprozeß mit Berücksichtigung des Zivilprozesses, Zürich 1974.

Hellwig, Albert: Psychologie und Vernehmungstechnik bei Tatbestandsermittlungen, 4. Auflage, Stuttgart 1951.

Heusler, A.: Die Grundlagen des Beweisrechts, AcP 62, 209 ff (1879).

Hohm, Karl-Heinz: Die Grundrechtsträgerschaft und "Grundrechtsmündigkeit" Minderjähriger am Beispiel öffentlicher Heimerziehung, NJW 1986, 3107 ff.

Jansen, Paul: FGG - Gesetz über die Angelegenheiten der freiwilligen Gerichtsbarkeit mit Nebengesetzen und bundes- und landesrechtlichen Ergänzungs- und Ausführungsvorschriften, Band I, 2. Auflage, Berlin 1969.

Jarass, Hans D.: Das allgemeine Persönlichkeitsrecht im Grundgesetz, NJW 1989, 857 ff.

Kahlke, Gerhard: Zur Beschaffenheit der Prozeßfähigkeit, ZZP 100, 10 ff (1987).

Kaissis, Athanassios: Die Verwertbarkeit materiell-rechtswidrig erlangter Beweismittel im Zivilprozeß, Frankfurt a.M., Bern, Las Vegas 1978.

Kallweit, Uwe: Die Prozeßförderungspflicht der Parteien und die Präklusion verspäteten Vorbringens nach der Vereinfachungsnovelle vom 3.12.1976, Frankfurt a.M., Bern, New York 1983.

Keidel, Theodor: Freiwillige Gerichtsbarkeit, Teil A, 12. neubearbeitete Auflage, München 1987 (zitiert: Keidel/Bearbeiter).

Kleinknecht, Theodor: Strafprozeßordnung, 39. Auflage fortgeführt von Karlheinz Meyer, München 1989 (zitiert: Kleinknecht/Meyer). 
Kleinknecht/Müller/Reitberger, KMR Kommentar zur Strafprozeßordnung, 7. Auflage neu bearbeitet von Hermann Müller, Walter Sax, Rainer Paulus, Frankfurt 1988 (zitiert: KMRBearbeiter).

Knippel, Siegmund, Zeugenbeweis ist schlechter Beweis, MDR 1980, $112 \mathrm{f}$.

Krönig, Ernst, Die Kunst der Beweiserhebung im Zivilprozeß, 2. Auflage, Hamburg 1947.

Krüger, Hildegard, Grundrechtsausübung durch Jugendliche (Grundrechtmündigkeit) und elterliche Gewalt, FamRZ 1956, 329 ff.

Lanzenauer, v. Rainer Haehling, Das Fragerecht der Parteien im Zivilprozeß, DRiZ 1966, $223 \mathrm{f}$.

Leipold, Dieter, Prozeßführungspflicht der Parteien und richterliche Verantwortung, ZZP 93, 237 ff (1980).

Lenckner, Theodor, Aussagepflicht, Schweigepflicht und Zeugnisverweigerungsrecht, NJW 1965, $321 \mathrm{ff}$.

- Grundsatzfragen des Beweisrechts, NJW 1965, $959 \mathrm{f}$.

Lent, Friedrich, Zeugenvernehmung einer nicht parteieidfähigen Prozeßpartei, ZZP 52, 14 ff (1927).

- Zur Unterscheidung von Lasten und Pflichten der Parteien im Zivilprozeß, ZZP 67, 344 ff (1954).

Lesch, Heiko, Die strafrechtliche Einwilligung beim HIV-Antikörpertest an Minderjährigen, NJW 1989, 2309 ff.

Löwe-Rosenberg, Die Strafprozessordnung und das Gerichtsverfassungsgesetz, Grosskommentar, 24. Auflage herausgegeben von Peter Riess, Berlin, New York 1988 (Zitiert: Löwe-Rosenberg-Bearbeiter).

Maunz-Dürig, Kommentar zum Grundgesetz, 2. Auflage, München 1987 (Zitiert: Bearbeiter in Maunz-Dürig).

Mes, Peter, Der Rechtsschutzanspruch, Mühlheim 1970.

Meyer, $H$., Das Verfahren bei Zeugnisverweigerung, ZZP 17, 459 ff (1882).

Meyke, Rolf, Die Funktion der Zeugenaussage im Zivilprozeß, NJW 1989, 2032 ff.

Mönkemöller, Otto: Psychologie und Psychopathologie der Aussage, Heidelberg 1930.

Moritz, Heinz Peter, Die (zivil-)rechtliche Stellung der Minderjährigen und Heranwachsenden innerhalb und außerhalb der Familie, Berlin 1989.

- Möglichkeit wirksamen Widerspruchs der Eltern gegen einen Schwangerschaftsabbruch bei der minderjährigen Tochter, JA 1981, 189 ff.

Mühl, Otto: Beweisbeschluß und Beweisinterlokut, ZZP 66, 165 ff (1953).

Müller-Luckmann, Elisabeth: Zur Befragung des Kindes im StrafprozeB. RdJB 1971, 217 f.

Münchener Kommentar zum Bürgerlichen Gesetzbuch, herausgegeben von Kurt Rebmann und Franz Jürgen Säcker, 2. Auflage München 1987, insbesondere Hinz, Manfred zu $\S \S 1626$ ff, Schwab, Dieter zu $\S 1795$ ff und Goerke, Paula zu $\S 1909$ ff (zitiert: MünchKomm-Bearbeiter).

Musielak, Hans-Joachim: Stadler, Max, Grundfragen des Beweisrechts, München 1984.

Niese, Werner: Doppelfunktionelle Prozeßhandlungen, Göttingen 1950. 
Orlowsky, Wedigo: Die Weigerungsrechte der minderjährigen Beweisperson im Strafprozeß, Tübingen 1973.

Panhuysen, Ursula: Die Untersuchung des Zeugen auf seine Glaubwürdigkeit - Ein Beitrag zur Stellung des Zeugen und Sachverständigen im Strafprozeß, Berlin 1964.

Pastor, Wilhelm: Die Unterlassungsvollstreckung nach § 890 ZPO - Die Zwangsvollstreckung von Unterlassungspflichten, 2. Auflage, Köln, Berlin, Bonn, München 1976.

Peters, Egbert: Die Verwertbarkeit rechtswidrig erlangter Beweismittel im Zivilprozeß, ZZP 76, 145 ff (1963).

- Zeugnisverweigerung als Beweisgrund, ZZP 77, 444 ff (1964).

Peters, Karl: Anmerkung zum Urteil des BGH 1 StS vom 27.10.70 - 1 StR 591/69, JR 1970, 307 ff.

- Beweisverbote im deutschen Strafverfahren, Gutachten für den 46. Deutschen Juristentag 1966, Band I, Teil 3 A, S. 91 ff, München, Berlin 1966 (zitiert: Gutachten).

- Strafprozeß, 3. Auflage Heidelberg, Karlsruhe 1981 (zitiert: Strafprozeß).

- Zeugenlüge und Prozeßausgang, Bonn 1939.

Petersen, Julius: Civilprozeßordnung für das Deutsche Reich, 5. Auflage, Lahr 1904.

Pickel, Harald: Die Bestellung eines Pflegers für prozeßunfähige Beteiligte im sozialgerichtlichen Verfahren, NJW 1965, $338 \mathrm{ff}$.

Prahm, Hayo: Die ärztlichpsychologische Glaubwürdigkeit Minderjähriger, Göttingen 1972.

Prestien, Hans-Christian: Die Stellung des Kindes im Rechtsstreit der Erwachsenen - Die gerichtliche Anhörung nach § 50b FGG aus der Sicht des Praktikers, RdJB 1988, 431 ff.

Reinicke, Gerhard: Die Krise der freien Beweiswürdigung oder Über die Schwierigkeit, einem Zeugen nicht zu glauben, MDR 1986, $630 \mathrm{ff}$.

Renner, Georg: Kann ein jugendlicher Zeuge nach $\S 50$ StPO zu einer Strafe verurteilt werden? Das Recht 1917, 378 f.

RGRK, Das Bürgerliche Gesetzbuch mit besonderer Berücksichtigung der Rechtsprechung des Reichsgerichts und des Bundesgerichtshofes, herausgegeben von den Mitgliedern des Bundesgerichtshofes, 12. Auflage, 63. Lieferung §§ $1626-1637$, Berlin, New York 1989 (zitiert: RGRK-Bearbeiter).

Rieß, Peter: Der Hauptinhalt des Ersten Gesetzes zur Reform des Strafverfahrensrechts (1. StVRG), NJW 1975, 81 ff.

Rieß, Peter / Hilger, Hans: Das neue Strafverfahrensrecht - Opferschutzgesetz und Strafverfahrensänderungsgesetz 1987, NStZ 1987, 145 ff, 204 ff.

Rixecker, Roland: Vermeintliche Randprobleme der Beschleunigung des Zivilprozesses, NJW 1984, 2135 ff.

Röhl, Hellmut: Prozeßfähigkeit Geisteskranker, JZ 1956, 309 ff.

Römer, Willhelm: Kinder und Jugendliche als Zeugen in Sittlichkeitsprozessen, RdJB 1971, 205 ff.

Roestel, Günther: Das verletzte Kind als Zeuge im StrafprozeB, RdJB 1971, $218 \mathrm{ff}$.

- Das Zeugnisverweigerungsrecht des Kindes im Strafverfahren gegen einen Angehörigen, NJW 1967, 967 f. 
Rosenberg, Leo: Das neue Zivilprozeßrecht nach dem Gesetz vom 27. Oktober 1933 (RGBI. I, 780), ZZP 58, 283 ff (1934).

Rosenberg, Leo / Schwab, Karl-Heinz: Zivilprozeßrecht, 14. Auflage, München 1986.

Rüßmann, Helmut: Die Zeugenvernehmung im Zivilprozeß, DRiZ 1985, 41 ff.

Rupp, Hans Heinrich: Beweisverbote im StrafprozeB in verfassungsrechtlicher Sicht, Gutachten für den 46. Deutschen Juristentag 1966, Band I, Teil 3 A, München, Berlin 1966.

Schaub, Dora: Zur Strafverfahrensproblematik bei minderjährigen Zeugen und Beschuldigten aus vormundschaftsrichterlicher Sicht, FamRZ 1966, 134 ff.

Schefold: Die Geschäfts- und Prozeßfähigkeit des Minderjährigen und das elterliche Vertretungsrecht, AcP 94, 305 ff (1903).

Schimansky, Herbert: Der Ausschluß des nicht beschuldigten Elternteils nach §52 Abs. 2 Satz 2 StPO, in: Festschrift für Gerd Pfeiffer, herausgegeben von Otto Friedrich Freiherr von Gamm, Peter Raisch und Klaus Tiedemann, Köln, Berlin, Bonn, München 1988 (zitiert: FS f. Pfeiffer).

Schimmack: Kinder und Jugendliche als Zeugen im Strafverfahren, JW 1924, 1667.

Schmidt, Eberhard: Kritische Anmerkungen zum Beschluß des Großen Senates für Strafsachen vom 8.12.1958 betreffend das Untersuchungsverweigerungsrecht nach StPO $\S 81 \mathrm{c}, \mathrm{JR} 1959,369 \mathrm{ff}$.

Schmidt, Richard: Lehrbuch des Deutschen Civilprozeßrechts, 2. Auflage, Leipzig 1906.

Schneider, Egon: Anmerkung zum Beschluß des OLG Köln vom 10.10.77 - Az: 16 W 111/77, NJW 1979, $987 \mathrm{f}$.

- Aussageverweigerung im Zivilprozeß, JurBüro 1970, 1021 ff.

- Beweis und Beweiswürdigung, 3. Auflage, München 1978.

- Die Ablehnung von Beweisanträgen im Zivilprozeß unter besonderer Berücksichtigung der höchstrichterlichen Rechtsprechung, ZZP 75, 173 ff (1962).

- Vernehmungskunst und Protokollierungstechnik, MDR 1965, 14 ff.

Schoene, Heiko: Das Zeugnisverweigerungsrecht des Kindes und das gesetzliche Vertretungsrecht der Eltern, NJW 1972, 930 ff.

Schönke, Adolf: Der Umfang der Beweisaufnahme im Zivilprozess, in: Festgabe für Leo Rosenberg, S. 217 ff, München, Berlin 1949 (zitiert: FS f. Rosenberg).

- Zivilprozeßrecht, 9. Auflage fortgeführt von Horst Schröder und Werner Niese, neu bearbeitet von Kurt Kuchinke, Karlsruhe 1969.

Schreiber, Rupert: Theorie des Beweiswertes für Beweismittel im Zivilprozeß, Berlin, Heidelberg, New York 1968.

Schubert, Werner: Protocolle der Commission zur Berathung einer allgemeinen Zivilprozeßordnung für die deutschen Bundesstaaten, Hannover 1864, neu herausgegeben von Werner Schubert, Frankfurt a.M. 1985 (zitiert: Protocolle).

Schumann, Ekkehard: Bundesverfassungsgericht, Grundgesetz und Zivilprozeß, ZZP 96, 137 ff (1983).

Schwab, Dieter: Familienrecht, 5. Auflage, München 1989. 
Schwab, Karl Heinz / Gottwald, Peter: Verfassung und Zivilprozeß, in: Effektiver Rechtsschutz und verfassungsmäBige Ordnung- Die Generalberichte zum VII. Internationalen Kongre $B$ für ProzeBrecht, Würzburg 1983, herausgegeben von Walter Habscheid, Bielefeld 1983.

Seuffert, Lothar: Zivilprozeßordnung für das Deutsche Reich nebst dem Einführungsgesetze, 2. Auflage, Nördlingen 1883.

Skupin, Karl-Heinz: Die Folgen beim Ausbleiben eines kindlichen oder jugendlichen Zeugen im Strafverfahren, MDR 1965, $865 \mathrm{ff}$.

Söllner, Rainer: Der Beweisantrag im Zivilprozeß, Erlangen, Nürnberg 1972.

- Zur Bedeutung der Erfahrungssätze bei der Ablehnung von Beweisanträgen, MDR 1988, $363 \mathrm{ff}$.

Soergel, Hans Theodor: Bürgerliches Gesetzbuch, neu herausgegeben von Wolfgang Siebert, Jürgen F. Bauer, 12. Auflage, Stuttgart, Berlin, Köln, Mainz 1988 (zitiert: Soergel-Bearbeiter).

Staudinger, Julius v.: Kommentar zum Bürgerlichen Gesetzbuch, herausgegeben von Günther Beitzke, 11. Auflage, Berlin 1966 (zitiert: Staudinger-Bearbeiter).

Stein-Jonas, Kommentar zur Zivilprozeßordnung, bearbeitet von Wolfgang Grunsky, Dieter Leipold, Wolfgang Münzberg, Peter Schlosser, Ekkehard Schumann, 20. Auflage, Tübingen 1988 (zitiert: Stein-Jonas-Bearbeiter).

Stephan: Bodo: Das Rechtsschutzbedürfnis, Berlin 1967.

Stern, William: Kinder und Jugendliche als Zeugen, DJZ 1910, $1001 \mathrm{ff}$.

- Zur Psychologie der Kinderaussagen, DJZ 1908, 51 ff.

Teplitzky, Otto: Der Beweisantrag im Zivilprozeß und seine Behandlung durch die Gerichte, JuS 1968, 71 ff.

Thomas, Heinz / Putzo, Hans: Zivilprozeßordnung, 15. Auflage, München 1987.

Trankell, Arne: Der Realitätsgehalt von Zeugenaussagen, Göttingen 1971.

Undeutsch, Udo: Forensische Psychologie, herausgegeben von Udo Undeutsch, Göttingen 1967.

Wach, Adolf: Vorträge über die Reichscivilprozessordnung, 2. Auflage, Bonn 1896.

Wacke, Andreas: Logische Paradoxien in antiker und moderner Jurisprudenz, in: Festschrift der Rechtswissenschaftlichen Fakultät zur 600-Jahr-Feier der Universität zu Köln, S. 325 ff, Köln, Berlin, Bonn, München 1989 (zitiert: FS der Universität Köln).

Wais, Edgar: Die Verwertbarkeit fehlerhaft erzielter Beweisergebnisse und rechtswidrig erlangter Beweismittel im Zivilprozeß, Tübingen 1966.

Waldner, Wolfgang: Aktuelle Probleme des rechtlichen Gehörs im Zivilprozeß, Erlangen 1983.

Walter, Gerhard: Anmerkung zum Urteil des BGH vom 3.11.87 - IV ZR 95/87, NJW 1988, $567 \mathrm{f}$.

- Freie Beweiswürdigung, Tübingen 1979.

Weber: Die Ausübung des Zeugnisverweigerungsrechtes im Strafprozeß durch einen Stellvertreter, MDR 1962, 169. 
Weigend, Thomas: Das Opferschutzgesetz - kleine Schritte zu welchem Ziel?, NJW 1987, 1170 ff.

Werner, Olaf: Verwertung rechtswidrig erlangter Beweismittel, NJW 1988, 993 ff.

Wetzell, Georg: System des ordentlichen Civilprozesses, 3. Auflage, Leipzig 1878.

Wieczorek, Bernhard: Zivilprozeßordnung und Nebengesetze, 2. Auflage, Berlin, New York 1976.

Wieser, Eberhard: Das Rechtsschutzinteresse des Klägers im Zivilprozeß, Bielefeld 1971.

Zöller, Richard: Zivilprozeßordnung, 16. Auflage bearbeitet von Reinhold Geimer, Köln 1990 (zitiert: Zöller-Bearbeiter). 


\section{Lebenslauf}

Geboren am 21.2.1962 in Ulm; verheiratet.

1981 Abitur am Ignaz-Kögler-Gymnasium in Landsberg am Lech.

Sommersemester 1982 bis Wintersemester 1986/87 Studium der Rechtswissenschaften an der Universität Regensburg.

Vom 1.12.1987 bis 31.3.1989 Zivildienst am Klinikum rechts der Isar der Technischen Universität München.

Referendarausbildung vom 1.4.1989 bis 15.1.1992.

Seit 7.2.1992 als Regierungsrat z.A. beim Bayerischen Staatsministerium der Finanzen. 